\title{
Die künftige Mechanisierung der polnischen Landwirtschaft unter besonderer Berücksichtigung der deutschen Erfahrungen mit Kooperationen
}

\author{
Dissertation \\ zur Erlangung des Doktorgrades \\ der Fakultät für Agrarwissenschaften \\ der Georg-August-Universität Göttingen
}

vorgelegt von

Rafał Chmielecki

geboren in Łódz

Göttingen, im Mai 2006 
D7

1. Referent: Prof. Dr. M. Köhne 2. Korreferent: Prof. Dr. W. Lücke

Tag der mündlichen Prüfung: 3. Juli 2006 


\section{Danksagung}

Die vorliegende Arbeit ist am Institut für Agrarökonomie der Georg-August-Universität entstanden. Zum Gelingen dieses Vorhabens hat eine Vielzahl von Personen beigetragen, bei denen ich mich an dieser Stelle herzlich bedanken möchte.

Mein ganz besonderer Dank gilt Herrn Prof. Dr. Manfred Köhne, der mich bereits im Jahr 2002 während meines ersten Aufenthalts in Göttingen zur Fortsetzung meiner Ausbildung in Deutschland motivierte und anschließend bei der Realisation des Forschungsvorhabens betreute. Von seinem Wissen, seiner Erfahrung und Ausgeglichenheit konnte ich in dieser Zeit unermesslich viel lernen.

Während der Bearbeitung des Themas war eine Zusammenarbeit mit vielen Personen und Institutionen außerhalb der Universität sowohl in Deutschland als auch in Polen erforderlich. Ihnen möchte ich, ohne die einzelnen Namen zu nennen meinen aufrichtigen Dank aussprechen.

Mein Dank gebührt weiterhin auch all jenen, die mich bei meiner Arbeit mit wertvollen Hinweisen und der Bereitschaft, die Mühe des Korrekturlesens auf sich zu nehmen, unterstützten.

An dieser Stelle danke ich auch allen Kolleginnen und Kollegen aus dem Institut. Sie haben nicht nur viel Geduld bei der Erörterung aller Probleme meiner Arbeit aufgebracht, sondern haben mir auch ermöglicht über den Feldrand der Wissenschaft hinaus zu schauen, die deutsche Sprache und Kultur näher kennen zu lernen.

Schließlich gilt mein großer Dank meinen Eltern, die mich mit unaufhörlichem Rückhalt bei meinen Studien unterstützten.

Ihnen sei diese Arbeit gewidmet. 


\section{Inhaltsverzeichnis}

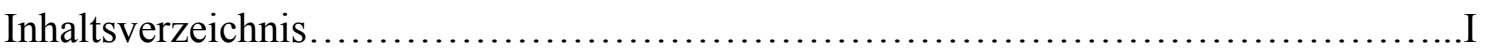

Verzeichnis der Abkürzungen.......................................................

Verzeichnis der Tabellen.........................................................

Verzeichnis der Abbildungen............................................... VIII

Verzeichnis der Übersichten.................................................. VIII

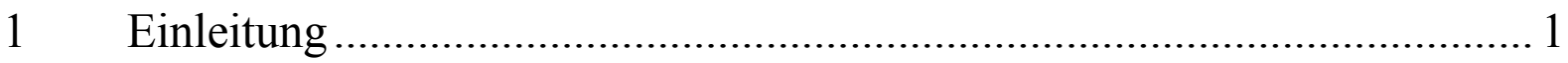

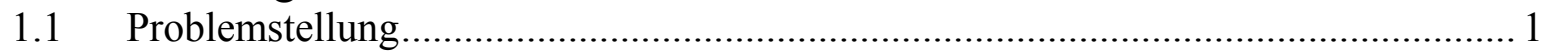

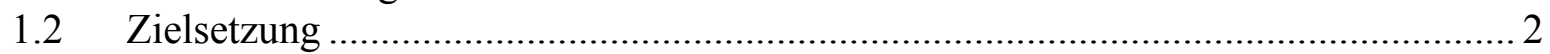

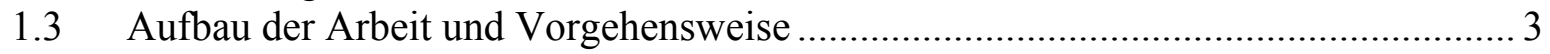

2 Entwicklung und Struktur der deutschen Landwirtschaft ...................... 6

2.1 Natürliche Standortbedingungen - Klima und Boden .......................................... 6

2.2 Landwirtschaftliche Nutzfläche und Betriebsgrößenstrukturen ................................ 7

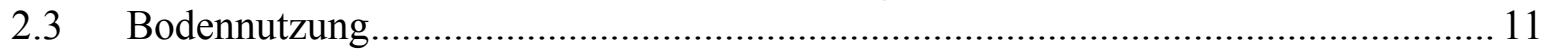

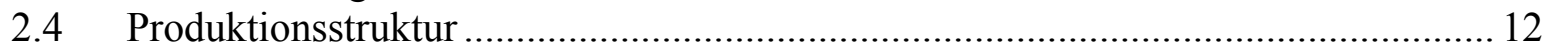

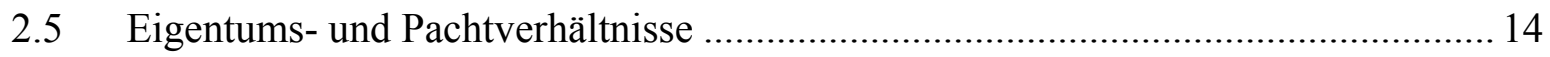

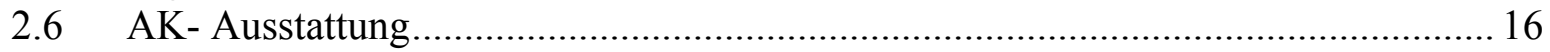

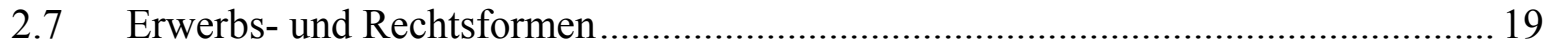

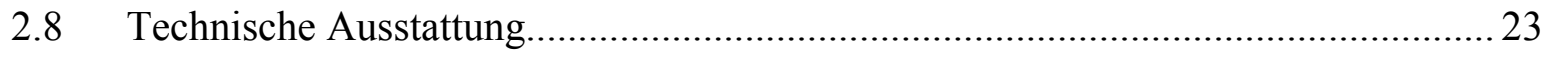

2.9 Direkte und indirekte Förderung des Modernisierungsprozesses ............................ 25

2.9.1 Förderung der einzelbetrieblichen Investitionen............................................26

2.9.2 Einkommensteuerwirksame Förderung....................................................... 29

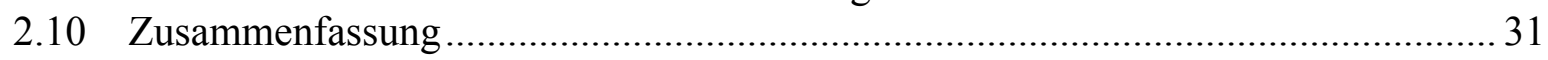

3 Entwicklung, Formen, Erfolge und Probleme der kooperativen

Maschinennutzung in Deutschland ........................................................ 33

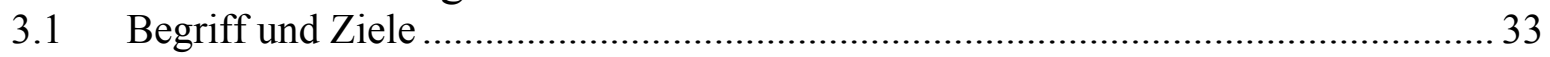

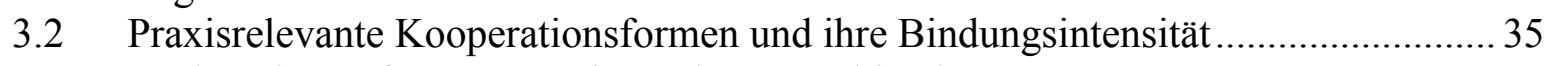

3.3 Rechtsrahmen für Kooperationen in Deutschland ................................................ 38

3.4 Entstehung, aktuelle Bedeutung und weitere Entwicklung verschiedener Formen der überbetrieblichen Zusammenarbeit beim Maschineneinsatz ............................ 43

3.4.1 Vertraglich geregelte Nachbarschaftshilfe .................................................... 45

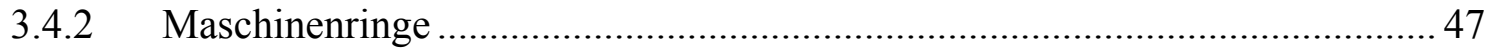

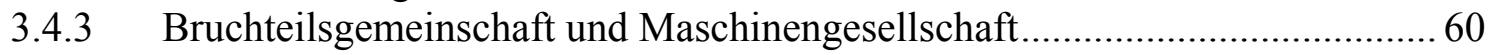

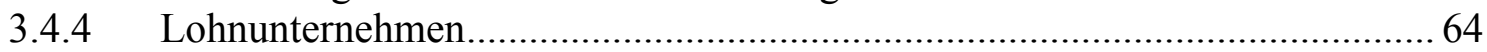

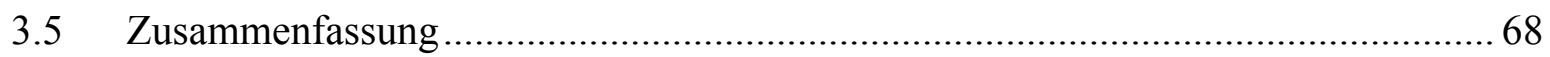

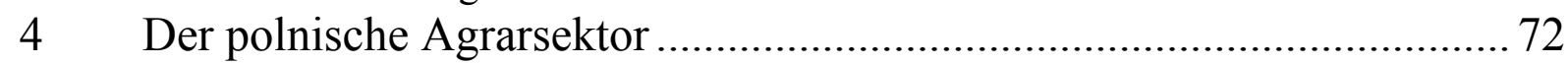

4.1 Natürliche Standortbedingungen - Klima und Boden .......................................... 72

4.2 Landwirtschaftliche Nutzfläche und Betriebsgrößenstrukturen............................... 72

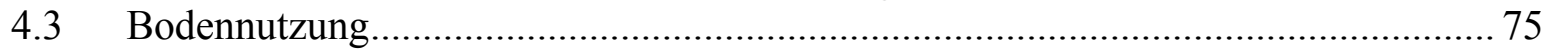

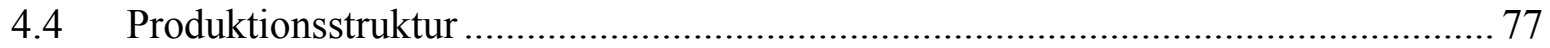

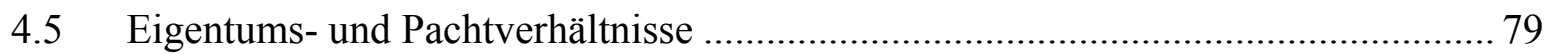

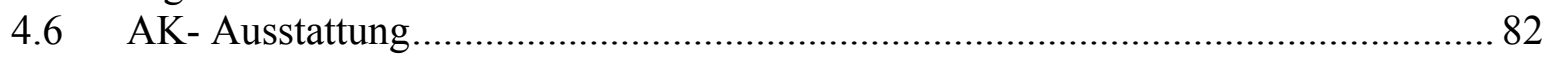

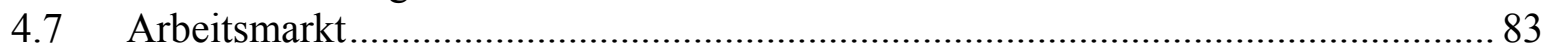

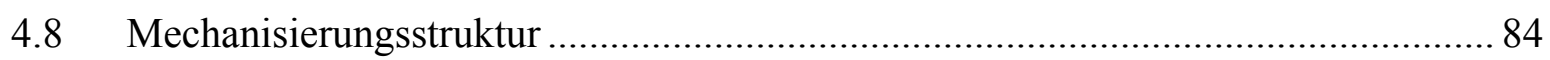

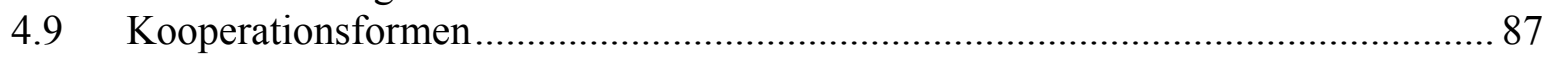

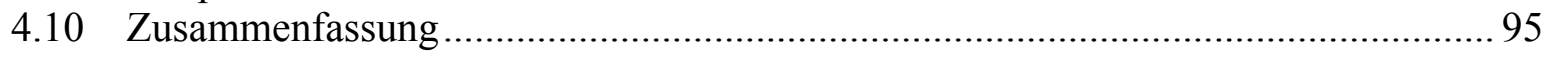




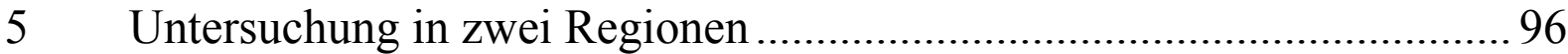

5.1 Abgrenzung der Untersuchungsregionen ..............................................................96

5.2 Charakteristik der Untersuchungsregion Ermland - Masuren ................................. 96

5.2.1 Natürliche Standortbedingungen................................................................96

5.2.2 Betriebsgrößenstruktur .............................................................................. 97

5.2.3 Bodennutzung und Struktur der Nutztierbestände ............................................. 97

5.2.4 Eigentums- und Pachtverhältnisse ............................................................. 100

5.2.5 Bevölkerungsstruktur und AK- Ausstattung ……………………………..... 100

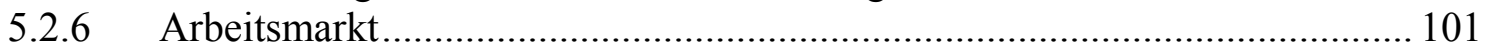

5.3 Charakteristik der Untersuchungsregion Lublin ................................................. 101

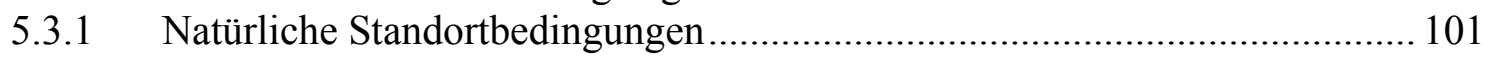

5.3.2 Betriebsgrößenstruktur ............................................................................ 102

5.3.3 Bodennutzung und Struktur der Nutztierbestände ........................................ 102

5.3.4 Eigentums- und Pachtverhältnisse ............................................................. 104

5.3.5 Bevölkerung und AK- Ausstattung ………………..................................... 104

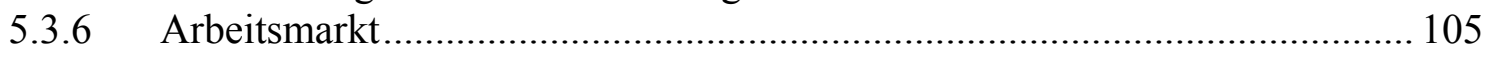

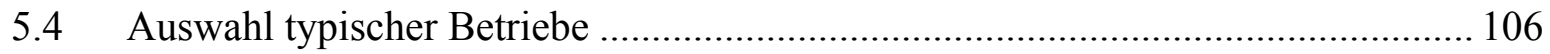

5.5 Betriebsbefragung ........................................................................................... 107

5.5.1 Befragungsergebnisse der Region Ermland - Masuren.................................... 107

5.5.2 Befragungsergebnisse der Region Lublin ................................................... 113

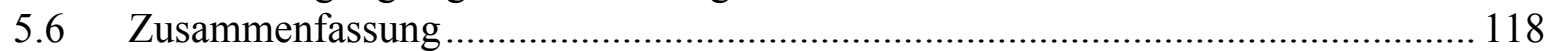

6 Potenzielle Mechanisierungspfade für die Untersuchungsregionen....... 120

6.1 Kosten des Einsatzes der vorhandenen Maschinen und die Bedeutung moderner Mechanisierung für die Betriebsentwicklung ………………………….............. 120

6.2 Geeignete Kooperationsformen........................................................................... 123

6.3 Auswahl zweckmäßiger Maschinen unter Berücksichtigung des Kooperationspotenzials .................................................................................. 128

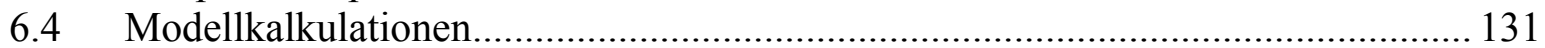

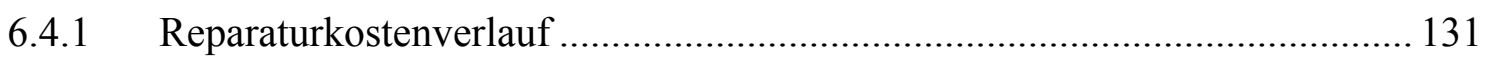

6.4.2 Restwertermittlung ................................................................................ 132

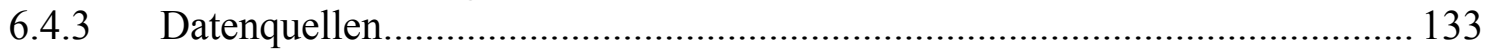

6.4.4 Kalkulationsmethodik .............................................................................. 134

6.4.5 Kalkulationen des Einsatzes neuer und gebrauchter Maschinen .................... 136

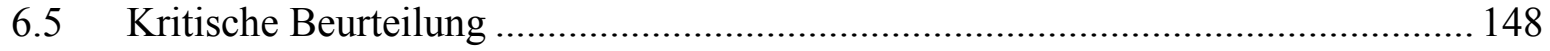

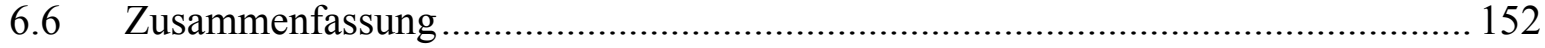

7 Formen der Investitionsfinanzierung und Ermittlung der optimalen

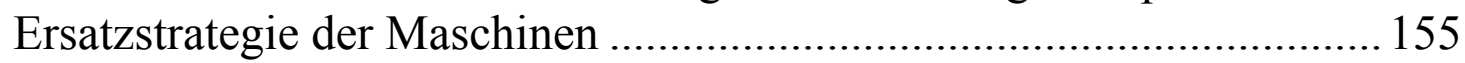

7.1 Vorteilhaftigkeitsanalyse der Finanzierung mittels zinsverbilligter und nicht

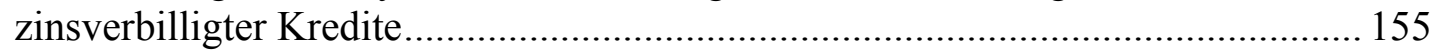

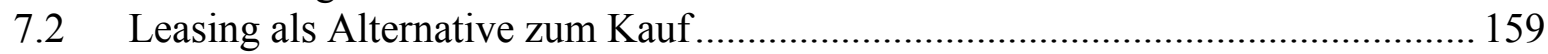

7.3 Gebrauchs- und zeitabhängiges Nutzungsdaueroptimum ..................................... 165

7.4 Beispielkalkulationen für die Ermittlung der optimalen Nutzungsdauer der

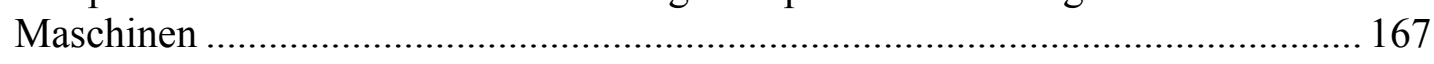

7.5 Zusammenfassung ............................................................................................ 170

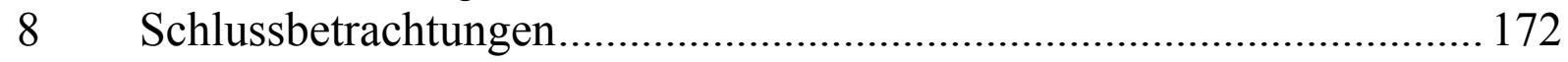

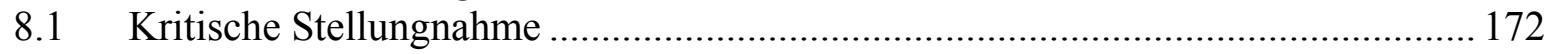

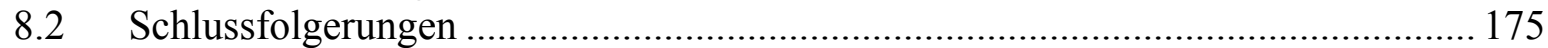

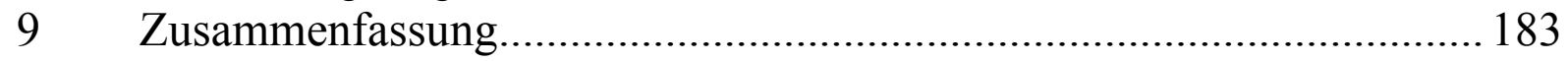

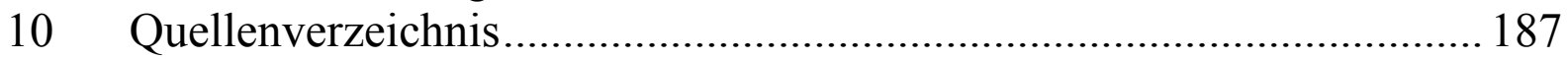

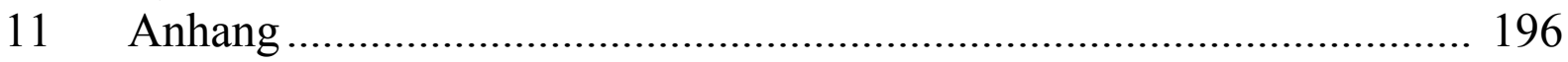




\section{Verzeichnis der Abkürzungen}

$\varnothing \quad$ durchschnittlich

A Anschaffungspreis

Abs. Absatz

AfA Absetzung für Abnutzung

AfaA Absetzung für außergewöhnliche Abnutzung

AFG Agrarförderungsgesetz

AG Aktiengesellschaft

AK Arbeitskraft

AKP Agrarinvestitionsförderungsprogramm

ANR Agencja Nieruchomości Rolnych (dt. Agentur für landwirtschaftliche Immobilien)

ARiMR Agencja Restrukturyzacji i Modernizacji Rolnictwa (dt. Agentur für Umstrukturierung und Modernisierung der Landwirtschaft)

ATKIS Amtliches Topographisch-Kartographisches Informationssystem

AWRSP Agencja własności rolnej skarbu państwa (dt. Agentur für landwirtschaftliches Staatseigentum)

AZ Ackerzahl

BayLwFöG bayerisches Landwirtschaftsförderungsgesetz

BewG Bewertungsgesetz

BGB Bürgerliches Gesetzbuch

BGBI Bundesgesetzblatt

BIP Bruttoinlandsprodukt

BLU Bundesverband der Lohnunternehmen

BMELV Bundesministerium für Ernährung, Landwirtschaft und Verbraucherschutz (ab 22.11.2005)

BML Bundesministerium für Ernährung, Landwirtschaft und Forsten (bis 21.01.2005)

BMR Bundesverband der Maschinenringe

BMVEL Bundesministerium für Verbraucherschutz, Ernährung und Landwirtschaft (ab 22.01.2005 bis 21.11.2005)

BVVG Bodenverwertungs- und -Verwaltungsgesellschaft

BZ Bodenzahl

bzw. beziehungsweise

${ }^{\circ} \mathrm{C} \quad$ Grad Celsius

ca. cirka

DF Diskontierungsfaktor

d.h. das heißt

DK Durchschnittskosten

DM Deutsche Mark

dt. deutsch

dt Dezitonne

DWD Deutscher Wetterdienst

EAGFL Europäischer Ausrichtungs- und Garantiefonds für die Landwirtschaft

EFP Einzelbetriebliches Investitionsförderungsprogramm

EGE europäische Größeneinheit

EStG Einkommensteuergesetz

EStR Einkommensteuer-Richtlinie

EUR Euro

et al. et alii (und andere) 


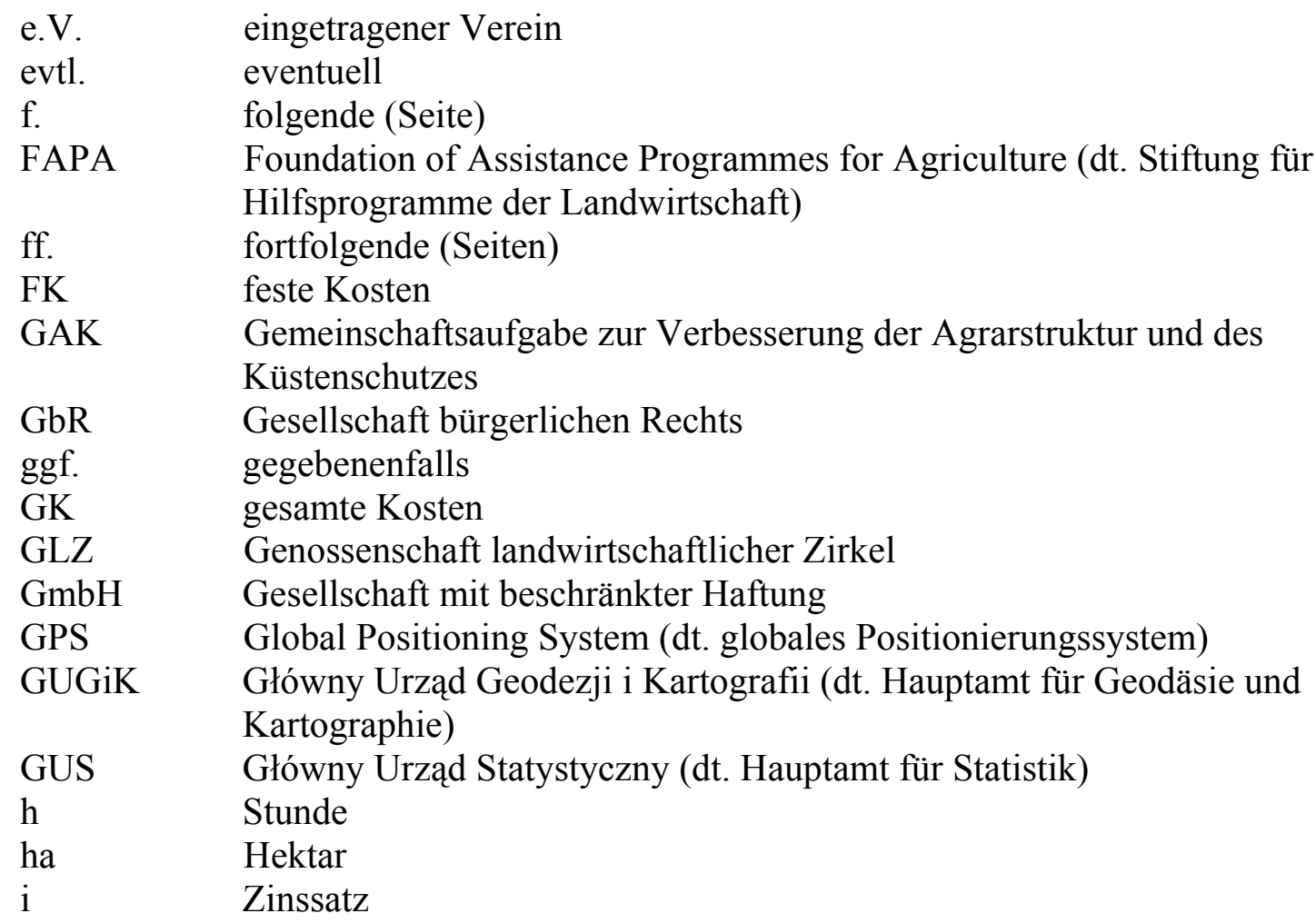

IBMER Instytut Budownictwa, Mechanizacji i Elektryfikacji Rolnictwa (dt. Institut für i.d.R. in der Regel

IERiGZ Instytut Ekonomiki Rolnictwa i Gospodarki Zywnościowej (dt. Institut für Landwirtschaftsökonomik und Ernährungswirtschaft)

i.e.S. im engeren Sinn

inkl. inklusive

i.S.d. $\quad$ im Sinne des

j jährliche Auslastung in ha oder $h$

JAE Jahresarbeitseinheit

KBM Kuratorium Bayerischer Maschinenringe

KG Kommanditgesellschaft

KK Kapitalkosten

$\mathrm{km}^{2} \quad$ Quadratkilometer

KTBL Kuratorium für Technik und Bauwesen in der Landwirtschaft

kW Kilowatt

KZKiOR Krajowy Związek Kółek i Organizacji Rolniczych (dt. Landesverband der Landwirte, Bauernzirkel und landwirtschaftlichen Organisationen)

L Liter

LBB mbH ländliche Betriebsgründungs- und Beratungsgesellschaft mit beschränkter Haftung

LBS landwirtschaftliche Beratungsstelle

LF landwirtschaftlich genutzte Fläche

LN landwirtschaftliche Nutzfläche

LPG landwirtschaftliche Produktionsgenossenschaft

LU Lohunternehmen

luf land- und forstwirtschaftlich

LVMR Landesverband der Maschinenringe

LWK Landwirtschaftskammer

LZ Landwirtschaftliche Zirkel 


\begin{tabular}{|c|c|}
\hline $\mathrm{m}^{2}$ & Quadratmeter \\
\hline Mio. & Million \\
\hline m.w.N. & mit weiteren Nachweisen \\
\hline MR & Maschinenring \\
\hline MRiRW & $\begin{array}{l}\text { Ministerstwo Rolnictwa i Rozwoju Wsi (dt. Ministerium für Landwirtschaft } \\
\text { und ländliche Entwicklung) }\end{array}$ \\
\hline MW & Marktwert \\
\hline $\mathrm{N}$ & Nutzungsdauer in Jahren \\
\hline $\mathrm{n}$ & Nutzungsdauer in Leistungseinheit ha, $\mathrm{h}$ \\
\hline $\mathrm{NN}$ & normales Null \\
\hline Nr. & Nummer \\
\hline o.g. & oben genannt \\
\hline p.a. & pro anno \\
\hline PHARE & $\begin{array}{l}\text { Poland and Hungary Aid for Restructuring of the Economies (dt. Polen und } \\
\text { Ungarn Hilfe zur Restrukturierung der Wirtschaft) }\end{array}$ \\
\hline PROW & $\begin{array}{l}\text { Program Rozwoju Obszarów Wiejskich (dt. Plan für die Entwicklung des } \\
\text { ländlichen Raums) }\end{array}$ \\
\hline PS & Pferdestärke \\
\hline RK & Reparaturkosten \\
\hline $\mathrm{RN}$ & Restnutzungsdauer \\
\hline RW & Restwert \\
\hline S. & Seite \\
\hline SDB & Standarddeckungsbeitrag \\
\hline sog. & so genannt \\
\hline SPO & Sektoralny Program Operacyjny (dt. sektorales operatives Programm) \\
\hline StMLF & Staatsministerium für Landwirtschaft und Forsten \\
\hline u.a. & unter anderem \\
\hline USt & Umsatzsteuer \\
\hline u.U. & unter Umständen \\
\hline ÜMV & überbetriebliche Maschinenverwendung \\
\hline v.a. & vor allem \\
\hline VE & Vieheinheit \\
\hline vgl. & vergleiche \\
\hline VK & variable Kosten \\
\hline vs. & versus \\
\hline WF & Wiedergewinnungsfaktor \\
\hline WG & Wirtschaftsgut \\
\hline WRSP & Własność Rolna Skarbu Państwa (dt. Landwirtschaftliches Staatseigentum) \\
\hline w.V. & wirtschaftlicher Verein \\
\hline z.B. & zum Beispiel \\
\hline$Z_{\text {eff. }}$ & Effektivzins \\
\hline $\mathrm{Zi}$ & Zinsanspruch \\
\hline ZL & Polnischer Zloty \\
\hline zzt. & zurzeit \\
\hline
\end{tabular}




\section{Verzeichnis der Tabellen}

Tabelle 2-1: Landwirtschaftliche Betriebe und ihre Flächen nach Bundesländern in den Jahren

1970-2005

Tabelle 2-2: Landwirtschaftliche Betriebe nach Größengruppen in den Jahren 1991-2005 ......... 10

Tabelle 2-3: Entwicklung der Flächenpacht in den Jahren 1971-2005 ................................... 15

Tabelle 2-4: Pacht- und Verkaufspreisrelationen nach Bundesländern im Jahr 2004 ................ 16

Tabelle 2-5: Erwerbstätige in Deutschland nach Wirtschaftsbereichen....................................... 17

Tabelle 2-6: Entwicklung der AK-Ausstattung in der Landwirtschaft in den Jahren 19702003

Tabelle 2-7: Relative Anteile der Betriebe mit außerbetrieblich erwerbstätigen Inhabern oder

ihren Familienangehörigen an der Anzahl aller landwirtschaftlichen Betriebe............. 21

Tabelle 2-8: Landwirtschaftliche Betriebe der Rechtsform Einzelunternehmen nach

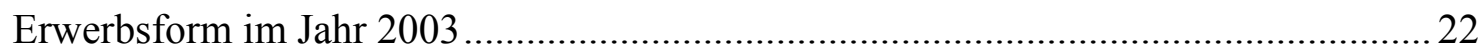

Tabelle 2-9: Landwirtschaftliche Betriebe nach Rechtsformen ................................................. 23

Tabelle 2-10: Struktur des Schlepperbestandes in den landwirtschaftlichen Betrieben des

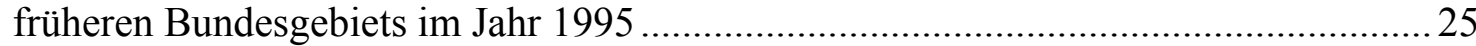

Tabelle 2-11: Motorleistung der Schlepper pro eine Flächeneinheit von 100 ha LF in

ausgewählten Ländern des früheren Bundesgebietes im Jahr 1995 .............................. 25

Tabelle 2-12: Beispielsdaten zur Wirkung der Sonderabschreibung bei konstanten Gewinnen .. 31

Tabelle 3-1: Entwicklung der MR im früheren Bundesgebiet in den Jahren 1960-2003 ........... 48

Tabelle 3-2: Maschinenringe in ausgewählten Bundesländern in den Jahren 1979-2003 ............52

Tabelle 3-3: Organisation der Betriebshilfe in den MR im Jahr 2003 ....................................... 56

Tabelle 3-4: Prozentualer Anteil der Auftraggeber und Auftragnehmer in den bayerischen

Maschinenringen in den Jahren 1992- 2005 in \% .....................................................5 59

Tabelle 3-5: Entwicklung der Maschinengemeinschaften in der Bundesrepublik Deutschland... 62

Tabelle 4-1: Struktur der landwirtschaftlichen Betriebe in den Jahren 1995- 2002 .................. 75

Tabelle 4-2: Struktur der Rinder- und Schweinebestände in den landwirtschaftlichen

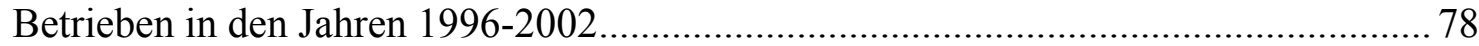

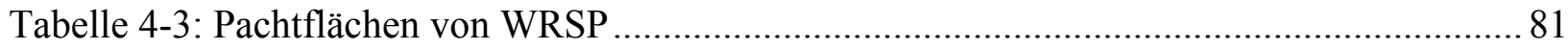

Tabelle 4-4: Durchschnittliche Boden- und Pachtpreise im Jahr 2004 ....................................... 81

Tabelle 4-5: Arbeitsaufwand in $1000 \mathrm{JAE}$ in landwirtschaftlichen Betrieben bestimmter

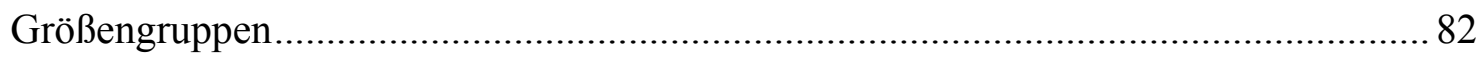

Tabelle 4-6: Status der mit den landwirtschaftlichen Betrieben verbundenen Personen nach

Art der Unterhaltsquelle in den Jahren 1996-2002 in \% ........................................... 83

Tabelle 4-7: Entwicklung der Anzahl der Erwerbstätigen in den Jahren 1990-2004 ................. 84

Tabelle 4-8: Zahl der Schlepper nach Motorleistung und Betriebsgröße ................................. 85

Tabelle 4-9: Die Auslastung der Schlepper und der Mähdrescher in den Jahren 1996-2002 ......86

Tabelle 4-10: Kredite des Branchenprogramms für gemeinsame Maschinennutzung in den

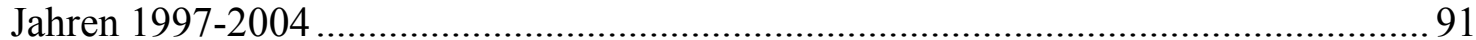

Tabelle 4-11: Förderungshöhe für Erzeugergruppen nach Jahren und realisiertem Umsatz ........ 93

Tabelle 4-12: Anzahl ausgewählter Erzeugergruppen in bestimmten Woiwodschaften ............. 93

Tabelle 4-13: Lohnunternehmen nach Woiwodschaften im Jahr 2005...................................... 94

Tabelle 5-1: Anzahl und LF der landwirtschaftlichen Betriebe der Woiwodschaft Ermland -

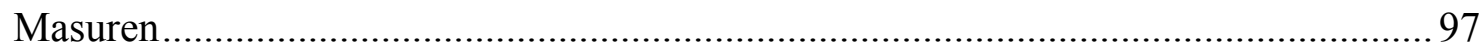

Tabelle 5-2: Anzahl der in den landwirtschaftlichen Betrieben beschäftigten Personen nach

Altersgruppen und Arbeitsumfang in Ermland - Masuren........................................ 101

Tabelle 5-3: Anzahl und LF der landwirtschaftlichen Betriebe der Woiwodschaft Lublin........ 102

Tabelle 5-4: Anzahl der in den landwirtschaftlichen Betrieben beschäftigten Personen nach

Altersgruppen und Arbeitsumfang in Lublin ................................................................ 105

Tabelle 5-5: Ausgewählte Ergebnisse der Befragung in Ermland - Masuren ............................ 110 
Tabelle 5-6: Akzeptanz verschiedner Kooperationsformen in den untersuchten Betrieben in Ermland- Masuren.

Tabelle 5-7: Ausgewählte Ergebnisse der Befragung in Lublin ....

Tabelle 5-8: Akzeptanz verschiedner Kooperationsformen in den untersuchten Betrieben in

Lublin

Tabelle 6-1: Anzahl der Betriebe nach der Höhe der Einsatzkosten der Schlepper und

Mähdrescher in beiden Untersuchungsregionen

Tabelle 6-2: Kostenkalkulation eines Schleppers mit einer Motorleistung von 90 PS (ZL) ...... 137

Tabelle 6-3: Kostenkalkulation eines Mähdreschers mit einer Motorleistung von 200 PS (ZL) 138

Tabelle 6-4: Verteilung der Anschaffungs- und Einsatzkosten des gebrauchten Mähdreschers mit 200 PS in einer Maschinengemeinschaft dreier Landwirte (ZL).

Tabelle 6-5: Verteilung des durch eine zusätzliche Auslastung des Mähdreschers mit 200 PS erzielbaren Einkommens (ZL)

Tabelle 6-6: Umverteilung der Kosten einer Maschinengemeinschaft beim Austritt eines

Mitglieds (ZL)

Tabelle 6-7: Kosten des Einsatzes des Mähdreschers in einer Maschinengemeinschaft dreier

Landwirte vs. Inanspruchnahme der Lohnmaschinenleistung (ZL)

Tabelle 6-8: Kostenkalkulation eines Mähdreschers mit einer Motorleistung von 130 PS (ZL) 142

Tabelle 6-9: Verteilung der Anschaffungs- und Einsatzkosten des gebrauchten Mähdreschers mit 130 PS in einer Maschinengemeinschaft zweier Landwirte (ZL) ....

Tabelle 6-10: Verteilung des durch eine zusätzliche Auslastung des Mähdreschers mit 130 PS erzielbaren Einkommens (ZL)

Tabelle 6-11: Kostenkalkulation eines Silierwagens mit einem Ladevolumen von $26 \mathrm{~m}^{3}$ (ZL) 145

Tabelle 6-12: Verteilung der Anschaffungs- und Einsatzkosten eines gemeinsamen gebrauchten Silierwagens nach Einsatzumfang (ZL)

Tabelle 6-13: Kostenkalkulation eines dreireihigen Rübenbunkerköpfroders (ZL) ................. 147

Tabelle 6-14: Verteilung der Anschaffungs- und Einsatzkosten eines gemeinsamen

Rübenbunkerköpfroders nach dem Einsatzumfang (ZL)....

Tabelle 7-1: Beispielsrechnung für Investitionsfinanzierung eines Schleppers (ZL) ............... 158

Tabelle 7-2: Vergleich der Finanzierung mithilfe von Kredit und Finanzierungsleasing (ZL) .. 161

Tabelle 7-3: Grunddaten zur Ermittlung der Steuerersparnis beim Kauf (ZL). 162

Tabelle 7-4: Beispiel zur Ermittlung der optimalen Nutzungsdauer eines Schleppers mithilfe der Durchschnittskostenmethode

Tabelle 7-5: Beispiel zur Ermittlung der optimalen Nutzungsdauer eines Schleppers mithilfe der Grenzkostenmethode für drei Nutzungsjahre und ein weiteres Nutzungsjahr. 


\section{Verzeichnis der Abbildungen}

Abbildung 2-1: Relative Anteile einzelner Betriebsgrößengruppen an der gesamten

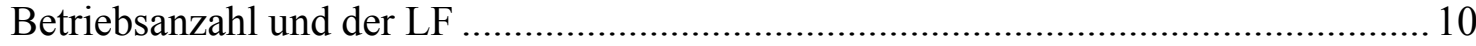

Abbildung 2-2: Relative Anteile der Anbauflächen an dem Ackerland .................................... 11

Abbildung 2-3: Relative Anteile der Nutzungsarten an der LF nach Bundesländern.................. 12

Abbildung 2-4: Relative Anteile der Betriebe bestimmter Größengruppen an den Rinder- und

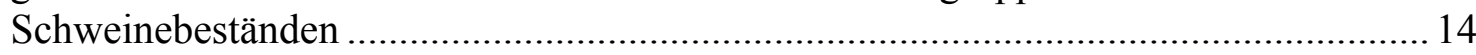

Abbildung 2-5: Arbeitsleistung in AK- Einheiten pro 100 ha LF nach Bundesländern im Jahr 2003

Abbildung 2-6: Die Entwicklung der Motorleistung der Schlepper in den landwirtschaftlichen

Betrieben des früheren Bundesgebiets in den Jahren 1970-1995 …............................24

Abbildung 3-1: Rechtsformen für kooperative Unternehmen............................................... 39

Abbildung 3-2: Anteile der Tätigkeitsbereiche an der gesamten Arbeitszeit der Mitarbeiter

(inkl. Geschäftsführer) in den bayerischen MR ......................................................... 58

Abbildung 4-1: Regionale Unterschiede in der Betriebsgrößenstruktur der

landwirtschaftlichen Betriebe in Polen

Abbildung 4-2: Relative Anteile einzelner Betriebsgrößengruppen an der gesamten LF und

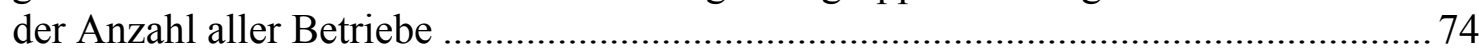

Abbildung 4-3: Relative Anteile der Anbauflächen an den Ackerflächen im Jahr 2002 ............. 76

Abbildung 4-4: Relative Anteile der Flächennutzungsarten nach Woiwodschaften ................... 77

Abbildung 4-5: Verteilung der Rinder- und Schweinebestände auf die einzelnen

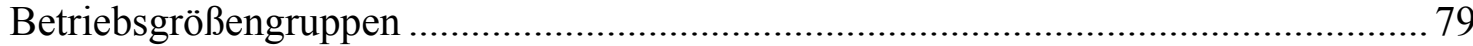

Abbildung 4-6: Kauf und Pacht der landwirtschaftlichen Flächen in den Jahren 1991-2005 ..... 80

Abbildung 5-1: Relative Anteile der Anbauflächen an dem Ackerland in Ermland -

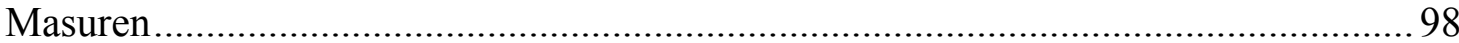

Abbildung 5-2: Relative Anteile bestimmter Betriebsgrößengruppen an den Rinder- und

Schweinebeständen in Ermland-Masuren ..................................................................... 99

Abbildung 5-3: Relative Anteile der Anbauflächen an dem Ackerland $\quad$ in Lublin........... 103

Abbildung 5-4: Relative Anteile bestimmter Betriebsgrößengruppen an den Rinder- und

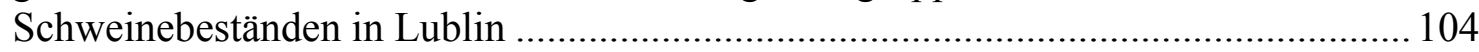

Abbildung 6-1: Kosten des Einsatzes des neuen und gebrauchten Mähdreschers mit 200 PS

in Abhängigkeit vom Leistungsumfang (ZL) ...................................................... 141

Abbildung 6-2: Kosten des Einsatzes eines neuen und gebrauchten Mähdreschers mit 130 PS

in Abhängigkeit vom Leistungsumfang (ZL) ....................................................... 144

Abbildung 6-3: Kosten des Einsatzes eines neuen und gebrauchten Silierwagens in

Abhängigkeit vom Leistungsumfang (ZL) ................................................................ 146

Abbildung 6-4: Kosten des Einsatzes des neuen und gebrauchten

Zuckerrübenbunkerköpfroders in Abhängigkeit vom Leistungsumfang (ZL)............ 148

\section{Verzeichnis der Übersichten}

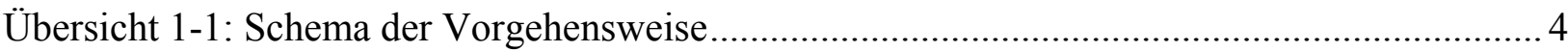

Übersicht 2-1: Die Bodenklassen nach dem deutschen und polnischen Bonitierungssystem ........ 7

Übersicht 3-1: Kooperationsformen nach der Art der gemeinsamen Aktivitäten........................ 35

Übersicht 3-2: Vereinsformen ........................................................................................ 42

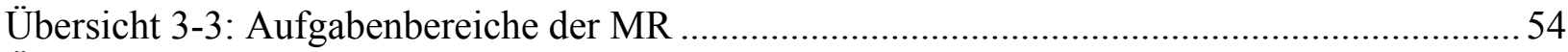

Übersicht 6-1: Bewertungskriterien zur Analyse der Entwicklungschancen ausgewählter

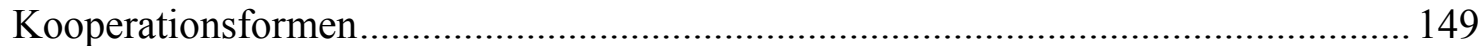

Übersicht 7-1: Kreditlinien für die Förderung der Maschineninvestitionen ............................. 156 


\section{Einleitung}

\subsection{Problemstellung}

Kennzeichnend für die polnische Landwirtschaft ist ein hoher Anteil von kleinen Familienbetrieben, die gemessen am Standard der westeuropäischen Landwirtschaft deutliche Defizite aufweisen. Diese betreffen neben den bereits angesprochenen ungünstigen Betriebsgrößenstrukturen, die niedrige Produktivität, die Produktionstechniken, die Organisation der Vermarktung der landwirtschaftlichen Erzeugnisse und nicht zuletzt auch die rückständige technische Ausstattung. Der ungünstige Zustand wird durch eine niedrige Kapitalausstattung der landwirtschaftlichen Betriebe zusätzlich verschärft.

Mit der Integration Polens in die Strukturen der EU ändern sich spürbar die Rahmenbedingungen. Um den neuen Herausforderungen $\mathrm{zu}$ gebühren, müssen in der polnischen Landwirtschaft erhebliche Anstrengungen unternommen werden. Ein wichtiges Gebiet auf dem dies geschehen muss, bildet die Mechanisierung der landwirtschaftlichen Betriebe. Die Modernisierung in diesem Bereich ist im Fall Polens aus folgenden Gründen dingend erforderlich:

- Die erforderliche Verbesserung der Einkommenssituation der ländlichen Bevölkerung und die Steigerung der Wettbewerbsfähigkeit der landwirtschaftlichen Betriebe setzen eine Erhöhung der Arbeitsproduktivität voraus, die ohne moderne Mechanisierung nicht erreichbar ist.

- Eine moderne Mechanisierung hilft die Naturalerträge steigern und zugleich die Verluste in der Außen- als auch in der Innenwirtschaft reduzieren. Auch auf diesem Wege ist die ökonomische Effizienz zu verbessern.

- Eine moderne Mechanisierung, insbesondere im kooperativen Gepräge, hilft die Produktionskosten senken, die Arbeitsbedingungen verbessern und die Freiräume für weitere Betriebsentwicklung schaffen. In diesem Sinn kann die Mechanisierung einen wesentlichen Beitrag zur Verbesserung der sozialen Bedingungen der landwirtschaftlichen Familien und einer Beschleunigung des notwendigen Strukturwandels leisten.

- Eine moderne Mechanisierung ist eine wichtige Voraussetzung für die Herstellung qualitativ hochwertiger Produkte. Da sich der Wettbewerb am Markt der 
landwirtschaftlichen Erzeugnisse u.a. über die Produktqualität vollzieht, müssen auch hier zügig Verbesserungen angestrebt werden.

- Bei weiterer Landwirtschaftsentwicklung sind die Einflüsse der landwirtschaftlichen Produktion auf die Umwelt zu bedenken. Der Einsatz moderner Technik hilft z.B. durch eine präzise Dosierung der Pflanzenschutzmittel oder Reduktion der Abgasemissionen den diesbezüglichen Anforderungen Rechnung tragen.

\subsection{Zielsetzung}

Die Einführung moderner Technik in die polnische Landwirtschaft kann aufgrund der angesprochenen Defizite, allen voran in Kapitalausstattung und ungünstigen Betriebsgrößenstrukturen, schwer fallen. Vor diesem Hintergrund ist das vorrangige Ziel der vorliegenden Arbeit, zu untersuchen, inwieweit die deutschen Erfahrungen mit der Modernisierung der Mechanisierung, insbesondere mit der Entwicklung verschiedener Formen der überbetrieblichen Zusammenarbeit, als potenzielle Orientierungshilfe für die polnische Landwirtschaft angewendet werden können. Eine entsprechende Untersuchung soll regional unterschiedliche Strukturen berücksichtigen. Daher soll der Pfad der künftigen Mechanisierung der polnischen Landwirtschaft exemplarisch anhand von ausgewählten landwirtschaftlichen Betrieben in zwei unterschiedlich strukturierten Regionen analysiert werden.

In dieser Analyse sind die folgenden Aspekte besonders zu berücksichtigen:

- Welche Entwicklungschancen resultieren aus der aktuellen Relation der in den landwirtschaftlichen Betrieben vorhandenen Produktionsfaktoren. In Regionen, in denen Kleinstrukturen vorherrschen und viele Arbeitskräfte vorliegen, wird die Modernisierung des Maschinenparks langsamer zu vollziehen sein als in Gebieten mit gegenteiligen Voraussetzungen.

- Welche Maschinengrößen sind in dem Entwicklungsprozess unter Berücksichtigung der regionalen Betriebsgrößenstrukturen zweckmäßig. Diesen Überlegungen muss eine Beurteilung der gegenwärtigen Mechanisierungsstruktur vorausgehen. Dabei sind sowohl der technische Zustand der vorhandenen Maschinen als auch die Möglichkeiten der Auslastung moderner Technik zu beachten. 
- Besondere Aufmerksamkeit ist hier den Entwicklungschancen bestimmter Kooperationsformen $\mathrm{zu}$ widmen. Denn im Rahmen der überbetrieblichen Zusammenarbeit kann die Verwirklichung der Modernisierungsinvestitionen erleichtert werden. Dabei handelt es sich neben der Kapitalbeschaffung auch um die Sicherung der erforderlichen Auslastung der neuen Maschinen.

Welche Finanzierungsformen kommen für die Ersatzinvestitionen in Maschinenpark in Betracht. Neben der Finanzierung mittels konventioneller Kredite ist an dieser Stelle die finanzielle Förderung des Staates und in einem weiteren Schritt auch das Leasing zu erörtern.

Neben ökonomischen sind auch psychologische und soziale Gesichtspunkte der Modernisierung $\mathrm{zu}$ berücksichtigen. Es muss daher bedacht werden, was und in welchem Tempo den Menschen an Modernisierung zugemutet werden kann.

- $\quad$ Im Rahmen dieser Arbeit ist der Frage nachzugehen, wie und ggf. inwieweit die Institutionen der landwirtschaftlichen Praxis z.B. die Beratungsstellen, die landwirtschaftlichen Verbände und nicht zuletzt auch die Regierung in den Modernisierungsprozess fördernd eingreifen können.

\subsection{Aufbau der Arbeit und Vorgehensweise}

Diese Arbeit besteht aus zwei Hauptteilen. Während die Ausführungen des ersten Teils der Struktur der deutschen Landwirtschaft und der Entwicklung verschiedener Formen der überbetrieblichen Zusammenarbeit im Bereich des Maschineneinsatzes gewidmet sind, gelten die Abhandlungen des zweiten Teils dem polnischen Agrarsektor. Die Vorgehensweise zur Bearbeitung der geschilderten Problemstellung wird in der Übersicht 1-1 graphisch dargestellt.

Es werden zunächst in Kapitel 2 die Entwicklungstendenzen der letzten Jahrzehnte in der deutschen Landwirtschaft aufgezeigt. Dies wird mit Hinblick auf die Arbeitskapazitäten, die Größenstrukturen, die Eigentums- und Pachtverhältnisse, die Mechanisierung und die Rechtsformen der landwirtschaftlichen Betriebe erfolgen. Die dabei gewonnenen Erkenntnisse werden zum einen als Hintergrund der Studie der Entwicklungsstadien verschiedener Kooperationsformen in Deutschland, zum anderen als Orientierungshilfe für die Analyse der Struktur des polnischen Agrarsektors herangezogen. 
In Kapitel 3 wird das Augenmerk auf die Entwicklung, Erfolge, Probleme und aktuelle Bedeutung der praxisrelevanten Kooperationsformen im Bereich des Maschineneinsatzes von der Nachbarschaftshilfe bis zur Zusammenarbeit mit den Lohunternehmen gerichtet. Um diese Problematik eingehend bearbeiten zu können, wird zunächst eine Auswertung der einschlägigen Literatur vorgenommen. In einem weiteren Schritt werden im Rahmen einer empirischen Studie Fachgespräche mit Experten aus der landwirtschaftlichen Praxis und dem landwirtschaftlichen Umfeld durchgeführt. Diese Personen werden nicht nur nach Vorteilen, sondern auch nach Defiziten, Problemen und evtl. Fehlentscheidungen in der vollzogenen Entwicklung befragt. Von besonderem Interesse sind hier auch die Empfehlungen der Experten für die Entwicklung der Mechanisierung der polnischen Landwirtschaft.

Übersicht 1-1: Schema der Vorgehensweise

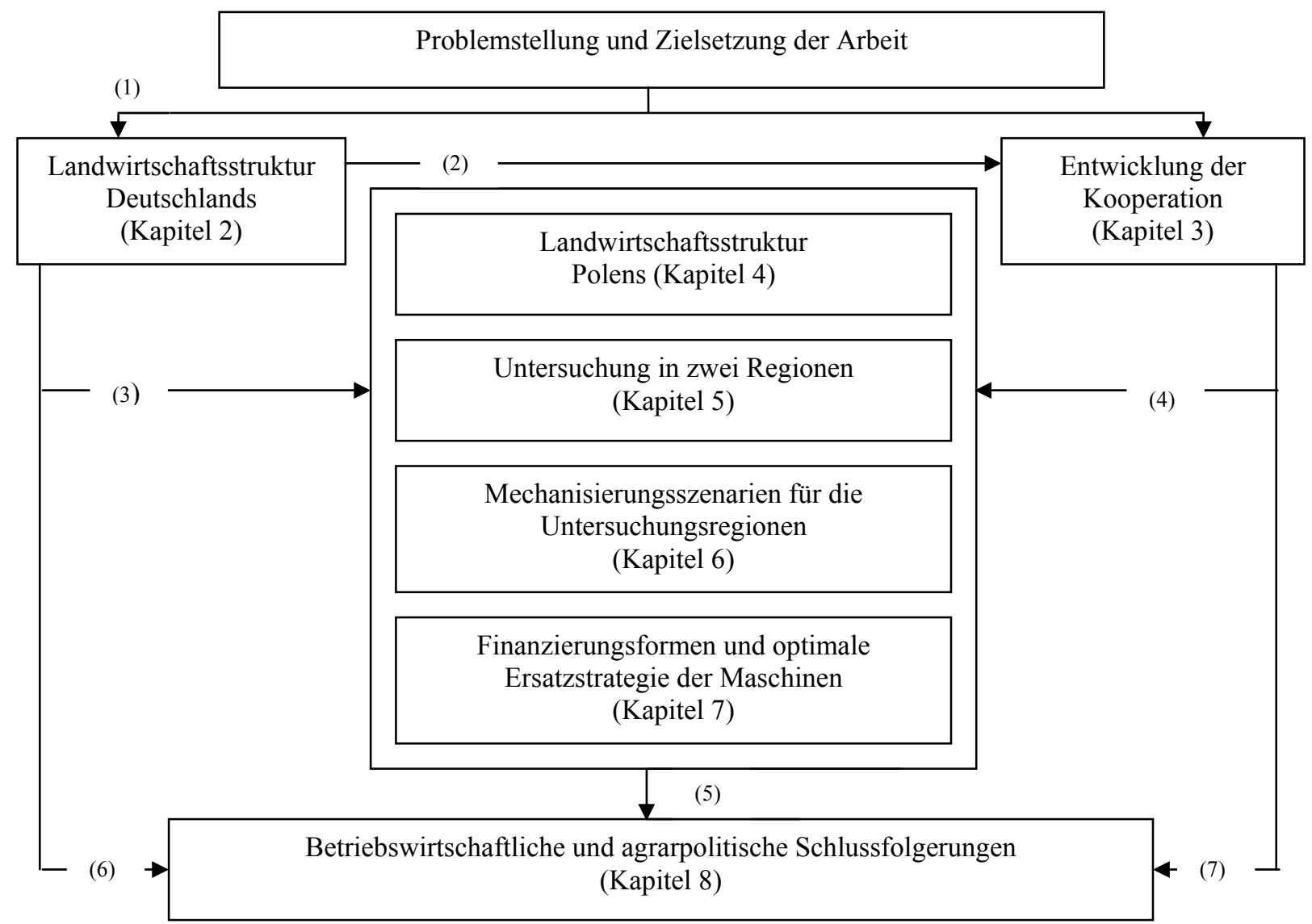

Quelle: eigene Darstellung

Da für die Formulierung der Verbesserungsvorschläge der künftigen Mechanisierung in Polen das Erkennen der Rahmenbedingungen unausweichlich ist, werden in Kapitel 4 die agrarstrukturellen Besonderheiten des polnischen Agrarsektors dargestellt. Den eigentlichen Hintergrund der empirischen Untersuchung in Polen bilden die Ergebnisse einer Befragung in 
ausgewählten landwirtschaftlichen Betrieben zwei Untersuchungsregionen. Die dabei gewonnenen Erkenntnisse werden in Kapitel 5 erläutert.

In Kapitel 6 werden eingangs die Aspekte der Entwicklung verschiedener Kooperationsformen, dann die Auswahl zweckmäßiger Maschinen für beide Untersuchungsregionen, anhand von exemplarischen Kostenkalkulationen aufgegriffen. Um den Praxisbezug der Arbeit zu sichern werden die Ergebnisse zur kritischen Beurteilung den Beratern und Landwirten vor Ort vorgelegt. Die diesbezüglichen Erkenntnisse werden abschließend im Rahmen einer Diskussion vorgestellt.

Das Kapitel 7 wird der Investitionsfinanzierung und der Ermittlung der optimalen Ersatzstrategie der Maschinen gewidmet.

In Kapitel 8 werden schließlich betriebswirtschaftliche und agrarpolitische Schlussfolgerungen formuliert. Dabei wird die Absicht verfolgt, die Ergebnisse der Arbeit so darzustellen, dass sie über die untersuchten Gebiete hinaus, auch für andere Standorte eine brauchbare Orientierungshilfe abgeben können. 


\section{Entwicklung und Struktur der deutschen Landwirtschaft}

In diesem Kapitel wird zunächst ein Einblick in wichtige Merkmale der deutschen Landwirtschaft vermittelt. Denn die Entwicklung der Mechanisierung wie auch der Kooperationsformen müssen vor diesem Hintergrund gesehen werden, um sie zu erklären und zu würdigen.

\subsection{Natürliche Standortbedingungen - Klima und Boden}

Das Klima Deutschlands wird als warmgemäßigtes Regenklima mit den Einflüssen der feuchten Luftmassen von Atlantik sowie der Winden von Osteuropa bezeichnet. Die mittlere Jahresdurchschnittstemperatur der Jahre 1960-1999 liegt bei 4,3 $3^{\circ} \mathrm{C}$ in Erzgebirge und $10,9^{\circ} \mathrm{C}$ in Heidelberg. Sie beträgt im Durchschnitt dieser Jahre $8,9^{\circ} \mathrm{C}$. Im Mittel fallen in Deutschland 600-790 mm/Jahr Niederschlag. Die jährlichen Niederschlagsmengen erreichen die Spannbreite von $417 \mathrm{~mm} / \mathrm{Jahr}$ in Artern (westlich von Halle, Saale) bis $2.450 \mathrm{~mm} / \mathrm{Jahr}$ in Balderschwang (Oberbayern). Die mittlere Sonnenscheindauer beträgt 1.528 Stunden pro Jahr. Die sonnigste Region ist Arkona/ Rügen, wo sich die Sonnenscheindauer insgesamt auf ca. 1.820 Stunden im Jahresmittel beläuft. Am wenigsten Sonnenstunden (1.264) entfallen auf die Region Trier im westlichen Teil von Rheinland Pfalz (vgl. DWD, PressEMITTEILUNG 2004).

An Böden kommen in Deutschland im Wesentlichen die Luvisole, die Parabraunerden, die gasenreichen Gleye sowie in niedrigen Anteilen die Schwarzerden der Bördengebiete vor. Die Bonitierung der landwirtschaftlichen Flächen erfolgt auf einer zahlenmäßigen Basis gemäß dem Bodenschätzungsgesetz aus dem Jahr 1934. Hierzu werden den einzelnen Ackerstandorten bestimmte Bodenzahlen (BZ) zugemessen, die im Allgemeinen die Ertragsfähigkeit der Böden widerspiegeln. Für die Bestimmung der BZ werden die Beschaffenheitsindikatoren wie: die Korngrößenklasse, das geologische Alter und die Zustandstufe der jeweiligen Böden herangezogen. Die Spannbreite der BZ reicht von 0 Punkten für die ertragsschwächsten Böden bis zu 100 Punkten für die Böden der besten Qualität. Unter zusätzlicher Berücksichtigung von Klima und Relief werden die sog. Ackerzahlen (AZ) bestimmt. Diese Zahlen werden durch Vornahme bestimmter Zu- oder Abrechnungen an den BZ ermittelt. Die AZ werden als Maßstab für die Bestimmung der durch den natürlichen Standort bedingten Ertragsfähigkeit der Böden herangezogen. Die geschilderte Methodik der Bonitierung für das Ackerland wird bei der Bewertung von 
Grünland, abgesehen von einzelnen Änderungen der Ackerschätzungsrahmen, im Prinzip beibehalten (vgl. SCHEFFER, SCHACHTSCHABEL 2002, S. 463-467).

Der überwiegende Teil der Böden in Deutschland entspricht mittlerer Qualität (50-65 Bodenpunkte). Die besten Böden von 80 und mehr Bodenpunkten sind selten und kommen nur in bestimmten Regionen (z.B. Hildesheimer und Magdeburger Börde, die Gäugebiete in der Nähe von Regensburg) vor. Zum Vergleich des deutschen und des polnischen Bodenbonitierungssystems ist auf die Übersicht 2-1 zu verweisen.

Übersicht 2-1: Die Bodenklassen nach dem deutschen und polnischen Bonitierungssystem

\begin{tabular}{|l|c|c|c|c|c|c|c|c|c|}
\hline Deutschland & Ackerzahl & $80-100$ & $65-80$ & $55-65$ & $45-55$ & $35-45$ & $30-35$ & $20-30$ & $0-20$ \\
\hline Polen & Bodenklasse & I & II & III a & III b & IV a & IV b & V & VI \\
\hline
\end{tabular}

\subsection{Landwirtschaftliche Nutzfläche und Betriebsgrößenstrukturen}

Die Landwirtschaftsfläche nahm im Jahr 2005 insgesamt 18,9 Mio. ha ein (53\% der Bodenfläche). Im Zeitablauf hat sie ständig abgenommen. Allein in der Zeitspanne 1993-2004 verringerte sich die Landwirtschaftsfläche um rund 608.000 ha oder 3,2\%. Dies entspricht dem in den fünfziger Jahren in den westlichen Bundesländern angefangenen Flächentransfer vom landwirtschaftlichen in die nicht landwirtschaftlichen Bereiche. Die Verkleinerung des Umfangs der Landwirtschaftsflächen wird im Wesentlichen zugunsten der Verkehrs- und Siedlungsflächen vollzogen. So nehmen die Flächen dieser Art allein in den Jahren 2001-2004 um 168.000 ha LF oder 115 ha/Tag zu. Im Vergleich mit den vergangenen Erhebungszyklen120 ha täglich zwischen 1992 und 1996 sowie 129 zwischen 1996 und 2000- hat sich der Zuwachs innerhalb dieser Flächenkategorie verlangsamt. Darüber hinaus ist in der Zeitspanne 2001-2004 ein leichter Anstieg der Waldflächen von 117.400 ha oder 1,1 \% zu verzeichnen. Während im Jahr 2001 der Anteil der Waldfläche an der Gesamtfläche 29,5 \% betrug, stieg er im Jahr 2004 auf ca. $30 \%$ an (vgl. STATiSTiSCHES BundeSAMT 2005, S. 6 ff.).

Der statistische Ausweis der Betriebsgrößen beginnt in Deutschland mit 2 ha LF. Demgemäß gab es im Jahr 2004 insgesamt 372.400 landwirtschaftliche Betriebe. Davon entfallen ca. $93 \%$ auf das frühere Bundesgebiet.

Der Strukturwandel in der Landwirtschaft der Bundesrepublik Deutschland ist seit Jahren durch eine Verringerung der Anzahl und eine gleichzeitige Vergrößerung der Flächenausstattung der bestehenden landwirtschaftlichen Betriebe gekennzeichnet. Während 
es auf dem früheren Bundesgebiet im Jahr 1970 über 1 Mio. landwirtschaftliche Betriebe mit einer Flächenausstattung unter 30 ha LF gab, verblieben im Jahr 2003 lediglich 235.500 Betriebe dieser Größengruppe. Die Anzahl der Betriebe mit einer Flächenausstattung von über 30 ha LF hat sich in der analysierten Periode nahezu verdoppelt (1970:72.717; 2003:125.000). Diese Entwicklung weist einen regional differenzierten Charakter auf. In Regionen, in denen geschichtlich bedingt ungünstige Kleinbetriebstrukturen vorherrschten, ging die Anzahl der einzelnen landwirtschaftlichen Betriebe weitaus schneller zurück, als in Regionen mit gegenteiligen Bedingungen. Die angesprochene Tendenz ist besonders deutlich beim Vergleich solcher Länder wie Baden - Württemberg, Rheinland - Pfalz oder Hessen mit solchen Ländern wie Niedersachsen oder Schleswig - Holstein. Während sich die Anzahl der landwirtschaftlichen Betriebe ab 2 ha LF zwischen 1970 und 2005 in Schleswig - Holstein um 22.500 verringert hat (55\% aller Betriebe), stellten in derselben Zeitspanne in Baden Württemberg insgesamt 103.500 Betriebe (63\% aller Betriebe) die Bewirtschaftung ein (siehe Tabelle 2-1). Auch heute sind die auf den Anfang der fünfziger Jahre zurückgehenden Unterschiede in der Entwicklung der Betriebsstruktur zwischen den nördlichen und südlichen Bundesländern existent. Während im Jahr 2005 in Schleswig - Holstein die Betriebe mit einer Flächenausstattung von weniger als 40 ha LF ungefähr $52 \%$ aller Betriebe ausmachten, zählten zu dieser Größengruppe in Hessen ca. 66 \% und in Bayern ca. 82 \% aller Betriebe. Die durchschnittliche Flächenausstattung eines landwirtschaftlichen Betriebes ist von Region zu Region unterschiedlich. Entfielen im Jahr 2005 auf einen landwirtschaftlichen Betrieb in Schleswig - Holstein ca. 55 ha LF, so wurden in demselben Jahr in einem Betrieb in Bayern im Schnitt ca. 25 ha LF bewirtschaftet. Im Durchschnitt der alten Bundesländer liegt die Flächenausstattung eines landwirtschaftlichen Betriebes bei ca. 34 ha LF.

Erheblich vom geschilderten Bild weicht die Betriebsgrößenstruktur in den östlichen Bundesländern ab. Im Jahr 2005 gab es in diesem Gebiet insgesamt 27.600 landwirtschaftliche Betriebe. Dies entspricht lediglich 7,6\% aller landwirtschaftlichen Betriebe Deutschlands. Die Betriebe mit einer Flächenausstattung von 2 bis 40 ha LF machen $55 \%$ aller Betriebe der östlichen Gebiete aus. In der Bewirtschaftung dieser Betriebsgruppe befinden sich jedoch lediglich $3 \%$ aller LF. Die größten Anteile an der LF entfallen auf die zahlenmäßig relativ kleine Gruppe der Betriebe mit einer Flächenausstattung von über 100 ha LF. Demzufolge verfügen 8.900 Betriebe dieses Gebiets über fast $93 \%$ der gesamten LF. Alles in allem beläuft sich die durchschnittliche Flächenausstattung eines landwirtschaftlichen Betriebes der östlichen Bundesländer auf 202 ha LF (vgl. BMELV 2006, S. 91). 
Tabelle 2-1: Landwirtschaftliche Betriebe und ihre Flächen nach Bundesländern in den Jahren 1970-2005

\begin{tabular}{|c|c|c|c|c|c|c|c|c|}
\hline & \multicolumn{3}{|c|}{$\begin{array}{c}\text { Zahl der Betriebe } \\
\text { in } 1000^{1}\end{array}$} & \multicolumn{3}{|c|}{$\begin{array}{c}\text { Fläche der Betriebe } \\
\text { in } 1000 \text { ha }\end{array}$} & \multicolumn{2}{|c|}{$\begin{array}{c}\varnothing \text { Betriebsgröße } \\
\text { in ha LF }\end{array}$} \\
\hline Früheres Bundesgebiet & 1970 & $\begin{array}{l}\text { davon } \\
<2 \text { ha }\end{array}$ & 2005 & 1970 & $\begin{array}{l}\text { davon } \\
<2 \text { ha }\end{array}$ & 2005 & 1970 & 2005 \\
\hline Baden- Württemberg & 253,4 & 89,3 & 60,6 & 1641 & 93,9 & 1446,5 & 6,5 & 24 \\
\hline Bayern & 357,7 & 57,2 & 129,4 & 3623 & 62 & 3265,7 & 10,1 & 25 \\
\hline Hessen & 108,4 & 32,5 & 23,6 & 857 & 34,7 & 771,9 & 8 & 33 \\
\hline Niedersachsen & 192,3 & 44,8 & 53,5 & 2828 & 47,1 & 2642,8 & 14,7 & 49 \\
\hline Nordrhein-Westfalen & 155,3 & 40 & 51,2 & 1765,3 & 42,9 & 1512,6 & 11,4 & 30 \\
\hline Rheinland- Pfalz & 114 & 36,6 & 26,3 & 817,3 & 39,3 & 713,6 & 7,1 & 27 \\
\hline Saarland & 13,3 & 6,5 & 1,7 & 85,7 & 6,2 & 78,6 & 6,4 & 46 \\
\hline Schleswig- Holstein & 49 & 8,3 & 18,2 & 1141,2 & 8,9 & 1007,9 & 23,2 & 55 \\
\hline Neue Länder & 1992 & & 2005 & 1992 & & 2005 & 1992 & 2005 \\
\hline Brandenburg & 4,8 & & 6,7 & 1229,4 & & 1337,7 & 256 & 200 \\
\hline Mecklenburg- Vorp. & 3,4 & & 5,1 & 1274,7 & & 1357,4 & 375 & 266 \\
\hline Sachsen & 4,1 & & 7,8 & 803,7 & & 913,1 & 196 & 117 \\
\hline Sachsen- Anhalt & 2,8 & & 4,9 & 1036,7 & & 1174,3 & 370,2 & 240 \\
\hline Thüringen & 3,5 & & 5,1 & 760,5 & & 799,1 & 217,3 & 157 \\
\hline
\end{tabular}

Quelle: Agrarbericht BMVEL, diverse Jahrgänge

Wird der Wandel in der Landwirtschaft in der Zeit nach der Wiedervereinigung, ohne Differenzierung nach einzelnen Ländern analysiert, so zeigt sich, dass in den Jahren 1991-2005 die Anzahl der Betriebe mit einer Flächenausstattung von über 2 ha LF um ca. 174.400 sank (siehe Tabelle 2-2). Es bedeutet einen Rückgang von insgesamt $32 \%$ aller Betriebe und entspricht einer durchschnittlichen Abnahmerate von 2,3\% pro Jahr. Diese Entwicklung wird vornehmlich durch die Verkleinerung der Anzahl der Betriebe mit einer Flächenausstattung von weniger als 50 ha LF vollzogen. Am deutlichsten nimmt die Zahl der Betriebe mit einer Flächenausstattung von 2-10 ha LF ab. Eine überdurchschnittliche Abnahmerate ist in den Betrieben mit einer Flächenausstattung von 20-30 ha LF zu erkennen. Die sog. Wachstumsschwelle, oberhalb derer die Betriebe wachsen oder unterhalb derer sie eingestellt werden, steigt im Laufe der Zeit an und liegt zzt. bei einer Größenordnung von über 75 ha LF.

\footnotetext{
${ }^{1}$ Aufgrund der unterschiedlichen Datenerfassung wurden für das Jahr 1970 für jedes Bundesland auch die Betriebe mit weniger als 2 ha LF ausgewiesen. Die Daten aus den Jahren 1992 und 2003 umfassen ausschließlich Betriebe mit einer Flächenausstattung ab 2 ha LF.
} 
Tabelle 2-2: Landwirtschaftliche Betriebe nach Größengruppen in den Jahren 1991-2005

\begin{tabular}{|c|c|c|c|c|c|}
\hline \multirow{2}{*}{$\begin{array}{c}\text { Betriebsgröße } \\
\text { in ha LF }\end{array}$} & 1991 & 2004 & 2005 & $\begin{array}{c}\text { 2004 gegen } \\
1991 \text { in \% }\end{array}$ & $\begin{array}{c}\text { 2005 gegen } \\
2004 \text { in \% }\end{array}$ \\
\cline { 2 - 3 } & \multicolumn{3}{|c|}{ Zahl der Betriebe in 1000} & $-40,8$ & $-2,4$ \\
\hline $2-10$ & 208,6 & 123,5 & 120,5 & $-40,4$ & $-0,54$ \\
$10-20$ & 123,3 & 73,4 & 73,0 & $-50,1$ & $-3,4$ \\
$20-30$ & 76,8 & 38,3 & 37,0 & $-30,6$ & $-2,2$ \\
$30-50$ & 76,2 & 52,8 & 51,6 & $+8,2$ & $-1,67$ \\
$50-75$ & 33 & 35,8 & 35,2 & $+69,3$ & $-0,52$ \\
$75-100$ & 11,2 & 19,1 & 19,0 & $+148,4$ & $+2,36$ \\
100 und mehr & 11,9 & 29,6 & 30,3 & & \\
\hline
\end{tabular}

Quelle: Statistisches Jahrbuch über Ernährung, Landwirtschaft und Forsten, diverse Jahrgänge

Die derzeitige Struktur der deutschen Landwirtschaft wird anhand der Abbildung 2-1 verdeutlicht, mit der die relativen Anteile einzelner Betriebsgrößengruppen an der gesamten Betriebsanzahl und der LF dargestellt sind. Es können daraus zwei Trendlinien abgeleitet werden und zwar eine, die die abnehmenden Anteile bestimmter Betriebsgrößengruppen an der gesamten Betriebsanzahl parallel zur Steigerung der Flächenausstattung abbildet und eine entgegengesetzte, die die wachsenden Anteile bestimmter Betriebsgrößengruppen an der gesamten LF charakterisiert. Dementsprechend sind die Betriebe mit einer Flächenausstattung von 2-30 ha LF zahlenmäßig am stärksten vertreten. Auf diese Größengruppe entfallen ca. $63 \%$ aller landwirtschaftlichen Betriebe. Zugleich bewirtschaften sie lediglich $16 \%$ der gesamten LF. Im Größenbereich 40-75 ha LF ist das Verhältnis ausgeglichen, d.h. auf ca. $16 \%$ aller Betriebe entfallen ungefähr $18 \%$ gesamter LF. Ein sehr bedeutender Flächenumfang gehört den Betrieben der Größengruppen von über 100 ha LF. Auf die Betriebe, die kaum $8 \%$ der gesamten Betriebsanzahl ausmachen, entfallen ca. $50 \%$ aller LF.

Abbildung 2-1: Relative Anteile einzelner Betriebsgrößengruppen an der gesamten Betriebsanzahl und der LF

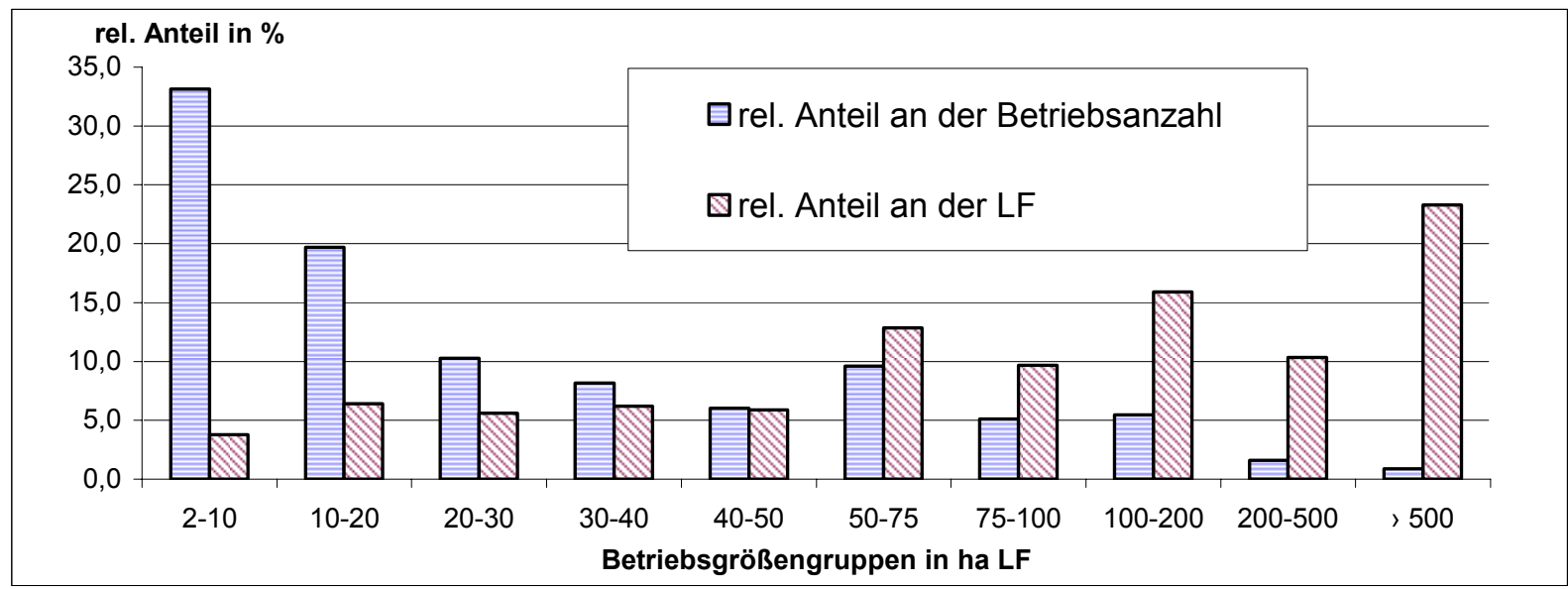

Quelle: Agrarbericht BMELV 2006 


\subsection{Bodennutzung}

Von den gesamten 17,02 Mio. ha LF Deutschlands entfallen ca. $70 \%$ auf das Ackerland und über $28 \%$ auf das Grünland. Ungefähr 1,2\% der LF wird für Garten- und Obstanlagen, Rebland, Baumschulen und Korbweiden beansprucht. Die Anteile verschiedener Anbauflächen an dem Ackerland sind in der Abbildung 2-2 graphisch dargestellt. Bei der Aufteilung der Getreideanbauflächen nach Fruchtarten rangiert an der ersten Stelle Weizen gefolgt von Gerste und Roggen. Auf diese Fruchtarten entfallen jeweils 45\%, $28 \%$ und $9 \%$ der gesamten Getreideanbaufläche.

Abbildung 2-2: Relative Anteile der Anbauflächen an dem Ackerland

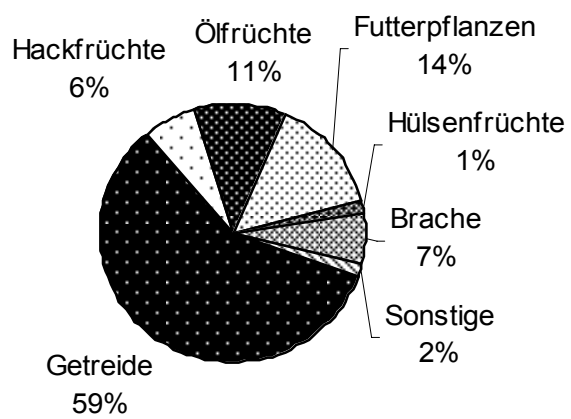

Quelle: Statistisches Bundesamt 2005

Die prozentuale Aufteilung der LF nach Bundesländern in die Nutzungskategorien zeigt eine unterschiedliche Produktionsstruktur in bestimmten Regionen. Wie der Abbildung 2-3 zu entnehmen ist, weisen den größten Anteil des Ackerlandes an der gesamten LF die östlichen Bundesländer, allen voran Sachsen - Anhalt auf. Dementsprechend wenige Flächen dieser Region werden als Dauergrünland oder in einer anderen Nutzungskategorie bewirtschaftet. In dem früheren Bundesgebiet ist der Anteil des Ackerlandes am höchsten in den nördlichen Regionen. Dabei handelt es sich namentlich um die Länder Schleswig-Holstein und Niedersachsen. Die höchsten Anteile des Dauergrünlands sind für die südlichen Regionen wie Bayern und Baden - Württemberg kennzeichnend. Der Anteil des Grünlandes an der gesamten LF dieser Länder bewegt sich auf dem Niveau von jeweils $35 \%$ und $39 \%$. Die größten Flächenanteile für Sonderkulturen sind für die Länder Rheinland-Pfalz, Baden - Württemberg und Hessen charakteristisch. 
Abbildung 2-3: Relative Anteile der Nutzungsarten an der LF nach Bundesländern

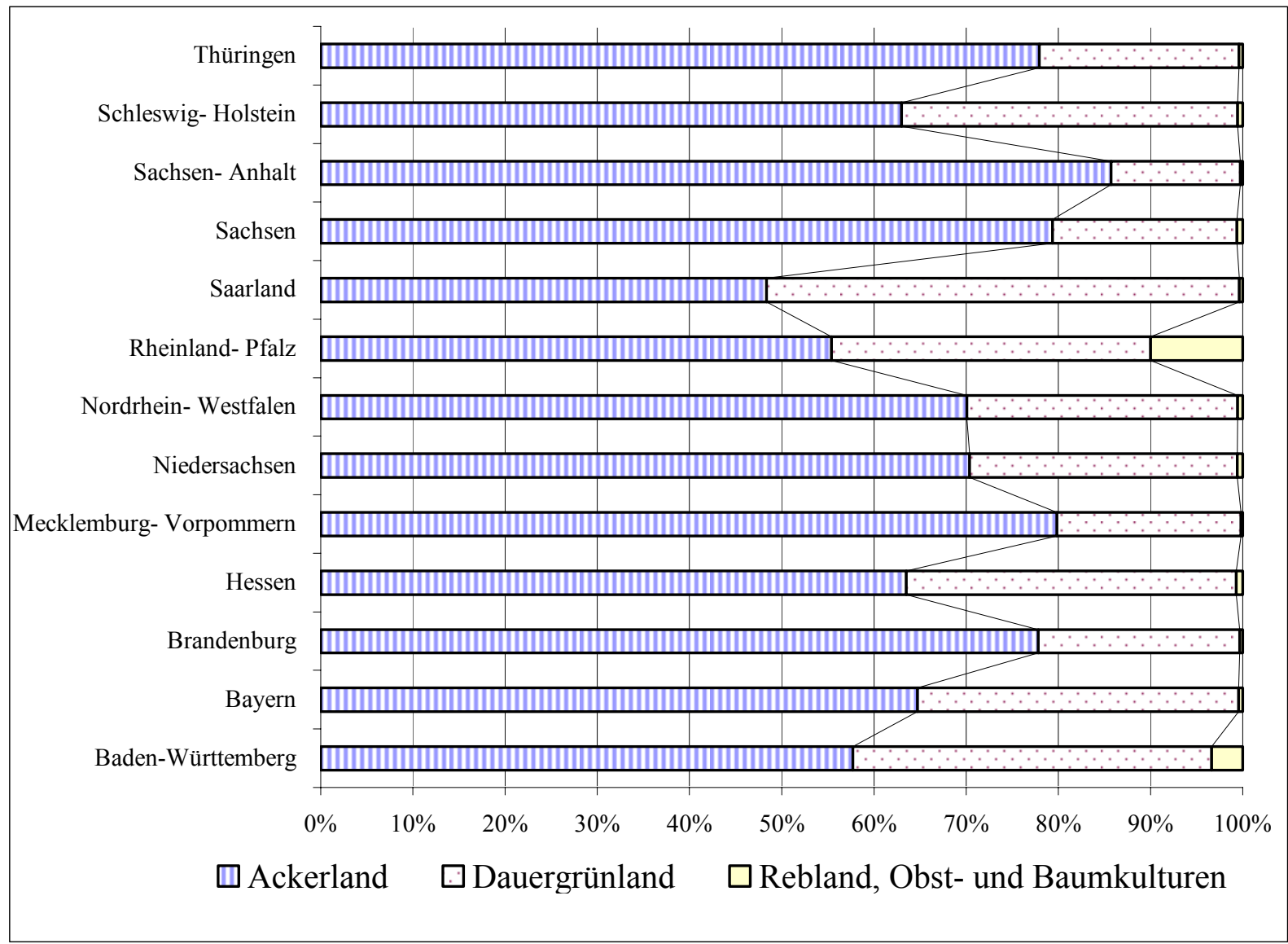

Quelle: Statistisches Bundesamt 2005

\subsection{Produktionsstruktur}

Bei der Klassifizierung der landwirtschaftlichen Betriebe nach der betriebswirtschaftlichen Ausrichtung dominieren in der deutschen Landwirtschaft die Futterbaubetriebe ${ }^{2}$. Dieser Produktionsausrichtung wurden im Jahr 2003 ca. 42\% aller Betriebe Deutschlands zugeordnet. Die höchsten Anteile der Futterbaubetriebe sind für die alten Bundesländer signifikant. In Schleswig-Holstein gehören ungefähr $60 \%$ aller Betriebe zu dieser Produktionsgruppe. Relativ stark vertreten sind die Betriebe dieser Produktionsrichtung in Bayern sowie in Niedersachsen, wo jeweils $50 \%$ und $45 \%$ aller Betriebe dieser Gruppe angehören. Die zweitgrößte Gruppe bilden die Ackerbaubetriebe. $\mathrm{Zu}$ dieser Produktionsrichtung zählten im Jahr 2003 ca. $23 \%$ aller landwirtschaftlichen Betriebe. Eine besondere Bedeutung haben die Ackerbaubetriebe in den östlichen Bundesländern. Demnach entsprechen dieser Produktionsrichtung in Sachsen-Anhalt ca. $55 \%$ und in

\footnotetext{
${ }^{2}$ Die Zuordnung eines landwirtschaftlichen Betriebes zu einer Ausrichtungsart erfolgt anhand des Beitrages der einzelnen Produktionszweige zu dem gesamten Standarddeckungsbeitrag (SDB) des Betriebes. Der SDB, der regionalisiert nach 38 Regionen (Regierungsbezirke) für 40 Produktionszweige der Bodennutzung und für 30 Tierhaltungsmerkmale ermittelt wird, entspricht der geldlichen Bruttoleistung abzüglich der entsprechenden variablen Spezialkosten (vgl. BMELV 2006, S. 110).
} 
Mecklenburg - Vorpommern ca. $50 \%$ aller Betriebe. Stark vertreten sind die Betriebe dieser Produktionsausrichtung auch in Bayern und Niedersachsen, wo jeweils $50 \%$ bzw. $45 \%$ aller Betriebe dieser Gruppe angehören. An der dritten Stelle befinden sich die Dauerkulturbetriebe (Wein- und Ostbau sowie sonstige Dauerkulturen). So wurden im Jahr 2003 ungefähr $9 \%$ aller Betriebe unter dieser Produktionsgruppe subsumiert. Der Anteil der Veredlungsbetriebe an der gesamten Betriebsanzahl erreichte im Jahr 2003 ca. $2 \%$. Die Betriebe dieser Produktionsausrichtung besitzen eine besondere Bedeutung in Niedersachsen und NordrheinWestfalen. Die Anzahl der Betriebe mit einer hohen Produktionszersplitterung, bei denen keine eindeutige Produktionsausrichtung bestimmt wird, ist verhältnismäßig klein. Die Anteile der Gemischtbetriebe an der Gesamtzahl aller Betriebe Deutschlands gehen nicht über $5 \%$ hinaus. Es ist dabei zu bemerken, dass sich zu $95 \%$ aller Gemischtbetriebe auf dem Gebiet der früheren Bundesländer, allen voran in Bayern und Baden- Württemberg, befinden.

Im Jahr 2003 gab es in Deutschland knapp 306.000 landwirtschaftliche Betriebe mit Viehhaltung ${ }^{3}$. Dies entspricht ca. $82 \%$ aller landwirtschaftlichen Betriebe. Der Rinderbestand betrug im Jahr 2003 insgesamt ca. 13 Mio. Tiere. Die meisten Rinderbestände entfallen auf die landwirtschaftlichen Betriebe in Bayern und Niedersachsen. Allein in diesen zwei Ländern befinden sich $47 \%$ des gesamten Rinderbestandes Deutschlands. Eine verhältnismäßig große Bedeutung besitzt die Rinderhaltung in Nordrhein-Westfalen, Schleswig - Holstein und in Baden - Württemberg. Die Bestände in diesen Ländern gehen jeweils über 1,1 Mio. Tiere hinaus. Gemessen an der Größe der Tierbestände einzelner Betriebe sind wesentliche Unterschiede in der Produktionsstruktur in bestimmten Bundesländern erkennbar. Während in Schleswig - Holstein auf einen Rinderhaltungsbetrieb durchschnittlich 120 Rinder entfallen, werden in einem Betrieb in Bayern im Schnitt 48 Rinder und in Baden - Württemberg 41 Rinder gehalten. Die größten Bestände befinden sich in den Betrieben in Mecklenburg - Vorpommern, wo auf einen Betrieb durchschnittlich 224 Rinder entfallen.

Der Schweinebestand wurde im Jahr 2003 auf insgesamt 26,3 Mio. beziffert. Eine besondere Bedeutung kommt der Schweinehaltung in Niedersachsen und Nordrhein - Westfallen zu. Allein in diesen zwei Ländern befinden sich über $53 \%$ des gesamten Schweinebestandes Deutschlands. Eine wesentliche Bedeutung hat die Schweinehaltung auch in Bayern, Baden Württemberg und Schleswig - Holstein. Ähnlich wie bei der Struktur der Rinderhaltung sind

\footnotetext{
${ }^{3}$ Darunter wird der gesamte Viehbestand aller Betriebe mit Rindern, Schweinen, Schafen, Pferden und Geflügel verstanden (vgl. STATISTISCHES BUNDESAMT 2003)
} 
auch im Fall der Schweinehaltung wesentliche Unterschiede in der Produktionsstruktur einzelner Länder erkennbar. Während in Schleswig-Holstein auf einen Betrieb durchschnittlich 562 Tiere entfallen, nimmt die Größe der Tierbestände im weiteren Binnenland erheblich ab. Werden in einem Schweinehaltungsbetrieb in Nordrhein - Westfalen durchschnittlich 374 Tiere gehalten, so befinden sich in einem Tierhaltungsbetrieb in Bayern im Schnitt 128 Tiere.

Bei Betrachtung der Viehbestände in der Aufteilung nach Betrieben bestimmter Größengruppen zeigt sich, dass die Tierhaltung in den kleineren Betrieben generell eine niedrige Bedeutung hat. So reicht z.B. der Anteil der Rinderbestände in den Betrieben mit einer Flächenausstattung bis zu 10 ha LF nicht über $4 \%$ hinaus. Den größten Anteil an der Tierproduktion haben die Betriebe im Bereich von 50-100 ha LF (siehe Abbildung 2-4).

Abbildung 2-4: Relative Anteile der Betriebe bestimmter Größengruppen an den Rinderund Schweinebeständen

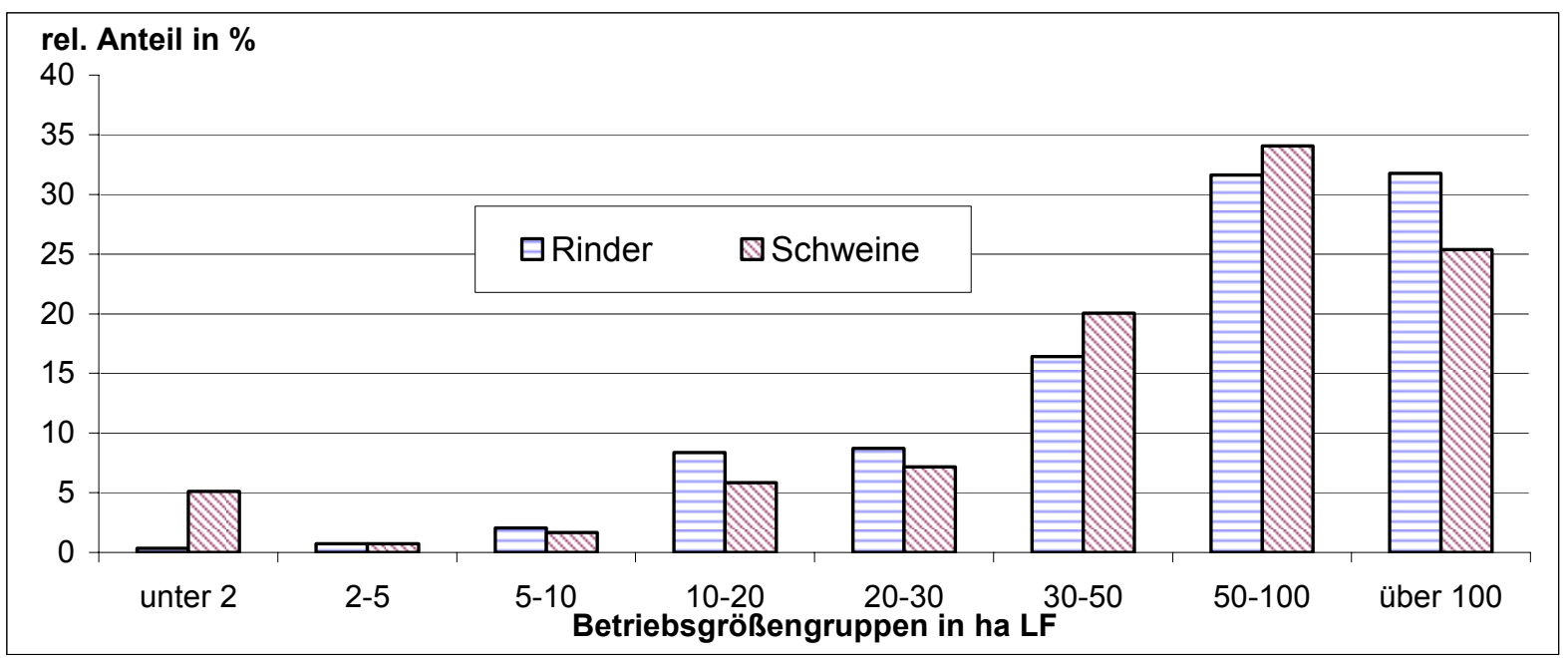

Quelle: Statistisches Bundesamt 2004

\subsection{Eigentums- und Pachtverhältnisse}

Eine besondere Bedeutung in der deutschen Landwirtschaft kommt der Flächenpacht zu. Im Jahr 2005 wurden nahezu $63 \%$ aller LF in Deutschland als Pachtflächen bewirtschaftet. An dieser Stelle sind wesentliche Unterschiede zwischen den westlichen und östlichen Regionen zu verzeichnen. Infolge der andauernd hohen Bodenpreise wird der Faktor Boden, bezogen auf die alten Bundesländer, häufig als langfristige Kapitalanlage betrachtet. Dies hat zur Folge, dass die Aufgabe der Bewirtschaftung des eigenen Betriebes häufig ohne Veräußerung der Flächen erfolgt. Hinzu kommt, dass infolge des bislang zugunsten der Pacht gestalteten Verhältnisses von Bodenkauf- und Pachtpreisen die erforderliche Betriebsvergrößerung durch 
Pacht weitaus günstiger ist, als der käufliche Erwerb des Bodens. Dies bestätigt auch die langfristige Entwicklung der Flächenpachtverhältnisse. Während im Jahr 1971 der Pachtflächenanteil an gesamter LF der landwirtschaftlichen Betriebe in den westlichen Ländern knapp $29 \%$ betrug, stieg er bis 2005 auf das Niveau von 53,5\% an. Für die angesprochene Zeitspanne signifikant ist ein Anstieg des Anteils der Betriebe mit gepachteten Flächen um ca. $10 \%$ (siehe Tabelle 2-3).

Tabelle 2-3: Entwicklung der Flächenpacht in den Jahren 1971-2005

\begin{tabular}{|c|c|c|c|c|c|}
\hline Früheres Bundesgebiet & 1971 & 1981 & 1991 & 2001 & 2005 \\
\hline Pachtflächenanteil in \% & 28,7 & 31,7 & 42,5 & 52 & 53,5 \\
\hline $\begin{array}{l}\text { Anteil der Betriebe mit } \\
\text { Pachtflächen in \% }\end{array}$ & 59,2 & 56,9 & 62,4 & 68,4 & 69,6 \\
\hline Neue Länder & 1991 & \multicolumn{2}{|c|}{1995} & 1999 & 2005 \\
\hline Pachtflächenanteil in \% & 77,5 & \multicolumn{2}{|c|}{90,1} & 88,1 & 81,2 \\
\hline $\begin{array}{l}\text { Anteil der Betriebe mit } \\
\text { Pachtflächen in } \%\end{array}$ & 51,2 & \multicolumn{2}{|c|}{59,7} & 69,3 & 69,6 \\
\hline
\end{tabular}

Quelle: Statistisches Jahrbuch über Ernährung, Landwirtschaft und Forsten, diverse Jahrgänge

In den neuen Bundesländern wird die Umstrukturierung der Landwirtschaft zum großen Teil auf der Grundlage der Pacht vollzogen (vgl. WinKLER 1996, S.7 ff.). Unter den Anbietern von Pachtflächen ist auf diesem Gebiet grundsätzlich zwischen der Treuhandanstalt bzw. Bodenverwertungs- und -verwaltungsgesellschaft $(\mathrm{BVVG})^{4}$ und privaten Bodeneigentümern zu unterscheiden. Die BVVG verwaltete im Jahr ihrer Gründung über 28 \% aller Acker- und Grünlandflächen der neuen Bundesländer. Bis Ende 2005 wurden auf dem Wege des Verkaufs 377.230 ha LF (ca. $27 \%$ der übernommenen LF) privatisiert. Ungefähr 626.470 ha LF des Bodenvolumens der BVVG werden zzt. verpachtet (vgl. BVVG 2006). Dies entspricht ca. $28 \%$ der gesamten gepachteten LF der neuen Bundesländer. Insgesamt werden zzt. ca. 81 \% aller LF der neuen Bundesländer als Pachtflächen bewirtschaftet. Dabei ist allerdings zu bemerken, dass die Entwicklung der Flächenpacht auf diesem Gebiet in den letzten Jahren, im Gegenteil zu Westdeutschland, rückläufig ist. Dies kann in erster Linie auf den Verkauf der Flächen der kleineren Betriebe zurückgeführt werden.

Nach wie vor bestehen im Pachtentgelt und Bodenpreis wesentliche Unterschiede zwischen dem früheren Bundesgebiet und den östlichen Bundesländern (siehe Tabelle 2-4). Die höchsten Pachtentgelte für das Ackerland werden in Nordrhein-Westfalen bezahlt.

\footnotetext{
${ }^{4}$ Die BVVG wurde im April 1992 ins Leben gerufen. Seit 1996 fungiert sie als eine Tochtergesellschaft der Bundesanstalt für vereinigungsbedingte Sonderaufgaben (BVS), die als Nachfolgerin der Treuhandanstalt auftritt. Seit 1996 ist die BVVG zivilrechtliche Eigentümerin der ihr übertragenen Flächen und Vermögenswerte, die ursprünglich einen Umfang von 1,4 Mio. ha Acker- und Grünland sowie 770.000 ha Waldland hatten (vgl. KlARE, Doll 1996, S. 67).
} 
Dementsprechend sind die Pachtpreise dieser Region im Schnitt dreimal höher als in den östlichen Bundesländern. Die Pacht des Dauergrünlands ist mit deutlichem Abstand am teuersten in Schleswig - Holstein. Im Vergleich zu den durchschnittlichen Pachtpreisen des Grünlandes in den östlichen Bundesländern sind hier die Pachtentgelte um das Dreifache höher. Die höchsten Verkaufspreise für landwirtschaftliche Flächen werden in Nordrhein Westfalen erzielt. Im Vergleich zu Sachsen ist der Boden hier ungefähr sechsmal teurer. Dabei ist allerdings zu bemerken, dass die genannten Preisrelationen zum einen wegen der wesentlichen Preisunterschiede in Abhängigkeit von Bodenlage und -qualität, zum anderen infolge der sich verändernden agrarpolitischen und betriebswirtschaftlichen Rahmenbedingungen, nur als Orientierungswerte und sehr zeitpunktbezogen betrachtet werden können.

Tabelle 2-4: Pacht- und Verkaufspreisrelationen nach Bundesländern im Jahr 2004

\begin{tabular}{|l|c|c|c|c|}
\hline \multirow{2}{*}{ Land } & $\begin{array}{c}\text { Pachtflächen } \\
\text { in \% der } \\
\text { gesamten LF }\end{array}$ & Pachtentgelt EUR/ ha Pachtfläche & $\begin{array}{c}\text { Verkaufswert } \\
\text { zum }\end{array}$ \\
\cline { 3 - 4 } & & & & $\begin{array}{c}\text { Aerkehrswert } \\
\text { je ha } \\
\text { Gesamtfläche }\end{array}$ \\
\hline Baden-Württemberg & 59 & 207 & 112 & 18385 \\
Bayern & 47 & 267 & 166 & 22392 \\
Brandenburg & 76 & 79 & 56 & 2452 \\
Hessen & 62 & 168 & 80 & 13200 \\
Mecklenburg-Vorpommern & 80 & 127 & 67 & 4344 \\
Niedersachsen & 50 & 311 & 183 & 13214 \\
Nordrhein- Westfalen & 52 & 347 & 180 & 25931 \\
Rheinland-Pfalz & 61 & 180 & 86 & 8358 \\
Saarland & 64 & 100 & 77 & 8507 \\
Sachsen & 86 & 119 & 63 & 3991 \\
Sachsen- Anhalt & 87 & 182 & 74 & 4615 \\
Schleswig- Holstein & 48 & 299 & 209 & 11053 \\
Thüringen & 91 & 131 & 57 & 4150 \\
Deutschland & 64 & & 121 & 9099 \\
\hline
\end{tabular}

Quelle: Statistisches Bundesamt 2005

\subsection{AK- Ausstattung}

Für den Strukturwandel in der westdeutschen Landwirtschaft ist eine seit den fünfziger Jahren andauernde Abwanderung der AK kennzeichnend. Die in der Nachkriegszeit einsetzende rapide Entwicklung der westdeutschen Volkswirtschaft bewirkte einen großen Bedarf an zusätzlichen Arbeitskräften. Die ländliche Bevölkerung bildete zu dieser Zeit ein bedeutendes Arbeitskräftepotenzial. Die Steigerung der Löhne in der Industrie, dem Dienstleistungssektor 
und dem Gewerbebereich sowie eine anhaltend ungünstige Scherenbewegung bei den Erzeugerpreisen für landwirtschaftliche Produkte gegenüber den Einkaufspreisen für landwirtschaftliche Betriebsmittel vergrößerte die Disparität in der Einkommenshöhe der in und außerhalb der Landwirtschaft beschäftigten Personen. Die Schwierigkeiten im Erreichen einer mit den außerlandwirtschaftlichen Bereichen vergleichbaren Entlohnung eigener Arbeit förderten zusätzlich den Übergang der Erwerbstätigen aus der Landwirtschaft in andere Wirtschaftsbereiche (vgl. KRÜLL 1990, S. 5).

Vor diesem Hintergrund ist bei der Untersuchung der Änderungen der AK- Ausstattung in der Landwirtschaft die gesamte volkswirtschaftliche Entwicklung Deutschlands zu berücksichtigen. Ein wesentliches Kennzeichen der entwickelten Volkswirtschaften ist, dass die Bedeutung des Agrarsektors für die Beschäftigung von Arbeitskräften, im Gegensatz zu anderen Wirtschaftsbereichen wie Handel und Verkehr oder Dienstleistung an Bedeutung verliert (vgl. FASTERDING, RIXEN 2005, S. 7). In Tabelle 2-5 wird die Entwicklung der Anzahl der Erwerbstätigen in verschiedenen Wirtschaftsbereichen aufgezeigt.

Tabelle 2-5: Erwerbstätige in Deutschland nach Wirtschaftsbereichen

\begin{tabular}{|c|c|c|c|c|c|}
\hline \multirow[t]{2}{*}{ Jahr } & \multirow{2}{*}{$\begin{array}{l}\text { Anzahl } \\
\text { in } 1000\end{array}$} & \multicolumn{4}{|c|}{ Anteile in Wirtschaftsbereichen in \% } \\
\hline & & $\begin{array}{c}\text { Land- und } \\
\text { Forstwirtschaft, } \\
\text { Fischerei }\end{array}$ & $\begin{array}{c}\text { Produzierendes } \\
\text { Gewerbe }\end{array}$ & $\begin{array}{c}\text { Handel und } \\
\text { Verkehr }\end{array}$ & $\begin{array}{c}\text { übrige } \\
\text { Wirtschaftsbereiche }\end{array}$ \\
\hline & & \multicolumn{4}{|c|}{ Früheres Bundesgebiet } \\
\hline 1960 & 26247 & 13,6 & 47,6 & 17,5 & 21,2 \\
\hline 1970 & 25668 & 8,5 & 48,8 & 17,4 & 25,2 \\
\hline 1980 & 27056 & 5,2 & 42,9 & 18,8 & 33,2 \\
\hline 1990 & 28486 & 3,5 & 38,8 & 19,1 & 38,6 \\
\hline \multirow[t]{2}{*}{1994} & 28316 & 2,9 & 35,1 & 19,8 & 42,3 \\
\hline & & \multicolumn{4}{|c|}{ Ost- und Westdeutschland } \\
\hline 1991 & 38454 & 4,0 & 36,7 & 24,3 & 35 \\
\hline 1994 & 37304 & 3,1 & 33,2 & 25 & 38,7 \\
\hline 1997 & 34208 & 2,7 & 31,1 & 25,1 & 41,1 \\
\hline 1999 & 38071 & 2,6 & 29,8 & 25,3 & 42,4 \\
\hline 2003 & 38314 & 2,4 & 27,2 & 25,4 & 45 \\
\hline
\end{tabular}

Quelle: Statistisches Jahrbuch über Ernährung, Landwirtschaft und Forsten, diverse Jahrgänge

Waren im Jahr 1970 im Bereich Landwirtschaft auf dem früheren Bundesgebiet insgesamt 2,7 Mio. Arbeitskräfte beschäftigt, so nahm diese Zahl bis zum Jahr 2005 auf ca. 1,1 Mio. AK $a b^{5}$. Die angesprochene Entwicklung wurde im Wesentlichen durch den Rückgang der Anzahl

\footnotetext{
${ }^{5}$ Zur Berechung der Nettowertschöpfung der AK wird die Arbeitsleistung der in der Landwirtschaft tätigen Personen in Jahresarbeitseinheiten (JAE) ausgedrückt. Dabei wird für Familienarbeitskräfte und ständige familienfremde AK ein Mindestarbeitseinsatz, je nach der Arbeitskräftekategorie, von 38 bis 42 Stunden angesetzt. Einer vollen JAE für nicht ständige AK entspricht eine Jahresarbeitsleistung von 220 vollen Arbeitstagen (vgl. BMELV 2006, S. 156).
} 
der Familienarbeitskräfte vollzogen. Einer Abnahmerate von ca. $68 \%$ in dieser AK-Gruppe entspricht eine Verminderung der Anzahl der familienfremden AK von insgesamt $21 \%$. Weiterhin zeigt sich bei der Analyse der statistischen Daten aus den Jahren 1999-2005, dass einer konstanten Abwanderung der Familien- AK eine Zunahme der familienfremden AK von 92.800 auf 102.900 um nahezu $11 \%$ gegenübersteht (siehe Tabelle 2-6).

Tabelle 2-6: Entwicklung der AK-Ausstattung in der Landwirtschaft in den Jahren 19702003

\begin{tabular}{|c|c|c|c|c|}
\hline Arbeitskräfte & Familienmitglieder & Familienfremde AK & $\begin{array}{c}\mathrm{AK} \\
\text { insgesamt }\end{array}$ & $\begin{array}{c}\text { Betriebliche } \\
\text { Arbeitsleistung } \\
\text { in } 1000\end{array}$ \\
\hline Früheres Bundesgebiet & \multicolumn{3}{|c|}{1000 Personen } & AK-Einheiten \\
\hline 1970 & 2475,8 & 130,6 & 2707,8 & 1525,6 \\
\hline 1980 & 1827,9 & 92,9 & 2006,1 & 986,7 \\
\hline 1990 & 1411,8 & 84,6 & 1569,7 & 748,7 \\
\hline 1997 & 999,8 & 92,8 & 1165,8 & 534,8 \\
\hline 1999 & 901,7 & 99,1 & 1268,2 & 499,6 \\
\hline 2001 & 822,1 & 99,0 & 1161,1 & 456,0 \\
\hline $2003^{6}$ & 783,7 & 102,8 & 1138,2 & 483,5 \\
\hline 2005 & 733,7 & 102,9 & 1099,7 & 453,7 \\
\hline \multicolumn{5}{|l|}{ Neue Länder } \\
\hline 1993 & 42,0 & 128,1 & 179,1 & 146,3 \\
\hline 1995 & 47,8 & 106,2 & 161,4 & 127,3 \\
\hline 1997 & 42,2 & 100,3 & 149,9 & 115,6 \\
\hline 1999 & 39,1 & 96,9 & 168,9 & 112,8 \\
\hline 2001 & 38,0 & 89,6 & 161,7 & 105,4 \\
\hline 2003 & 40,1 & 88,4 & 166,7 & 104,9 \\
\hline 2005 & 38,8 & 84,0 & 164,4 & 100,5 \\
\hline
\end{tabular}

Quelle: Agrarbericht BMVEL 2005

Anders stellt sich die Beschäftigungsstruktur in den neuen Bundesländern dar. Die Anzahl der in der Landwirtschaft dieser Länder beschäftigten Personen macht insgesamt lediglich ca. $15 \%$ aller AK der Landwirtschaft Deutschlands aus. Im Gegensatz zu dem früheren Bundesgebiet dominieren hier nach wie vor familienfremde Arbeitskräfte. So werden ca. $76 \%$ aller Beschäftigten dieser Gruppe zugeordnet. Der Strukturwandel im Bereich AKAusstattung weist in diesen Ländern einen anderen Charakter als der Wandel auf dem früheren Bundesgebiet auf. In den Jahren 1997-2003 wird in der Gruppe der familienfremden

\footnotetext{
${ }^{6}$ Aufgrund der Änderung des Erhebungskonzepts sind die Ergebnisse zur Anzahl der AK nur beschränkt, die Anzahl der Arbeitskräfteeinheiten nicht mit denen der anderen Jahre vergleichbar.
} 
AK ein Rückgang von insgesamt $12 \%$ verzeichnet. Zugleich sinkt die Anzahl der Beschäftigten der Gruppe der Familienmitglieder eher geringfügig (2,1\%). In den Jahren 2001-2003 zeichnet sich in diesen Regionen allerdings eine veränderte Tendenz ab. Während die Anzahl der familienfremden- AK nach wie vor reduziert wird, nimmt die Gruppe der Familien- AK von rund 38.000 auf 40.100 AK um 5,5 \% zu.

Aus dem Vergleich der Arbeitsleistung pro 100 ha LF in den landwirtschaftlichen Betrieben ergeben sich wesentliche Unterschiede zwischen einzelnen Bundesländern. Es sind an dieser Stelle sowohl erhebliche Differenzen zwischen den westlichen und östlichen Gebieten als auch das sog. Nord- Süd- Gefälle erkennbar. Hierzu ist auf die Abbildung 2-5 zu verweisen.

Abbildung 2-5: Arbeitsleistung in AK- Einheiten pro 100 ha LF nach Bundesländern im Jahr 2003

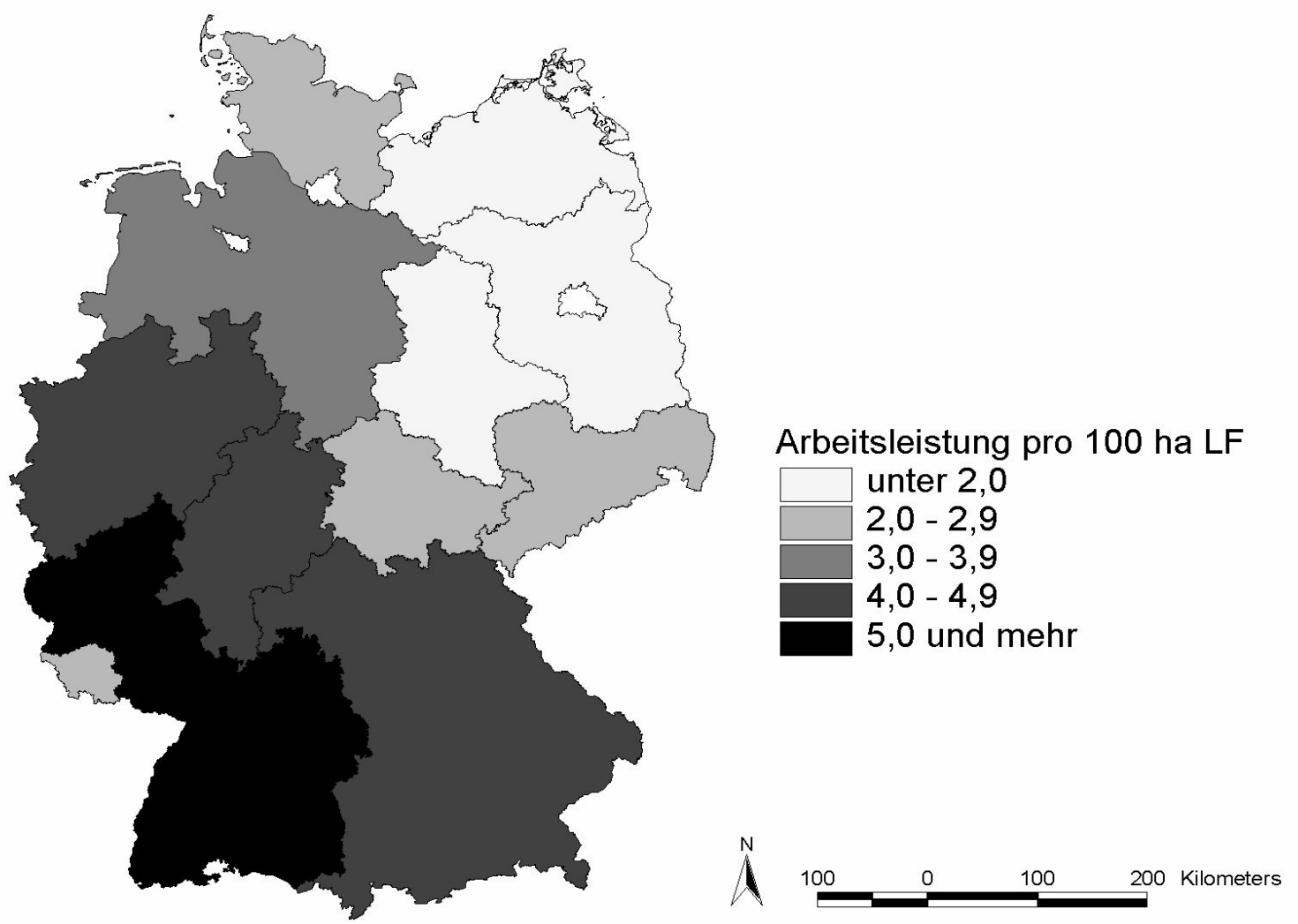

Quelle: Statistisches Bundesamt 2003; ATKIS VG 250, Bundesamt für Kartographie und Geodäsie 2003

\subsection{Erwerbs- und Rechtsformen}

Eine besondere Stellung in der deutschen Landwirtschaft nehmen die Nebenerwerbsbetriebe ein. Eine Analyse der statistischen Daten der letzten Jahrzehnte lässt erkennen, dass der Rückgang der Anzahl der landwirtschaftlichen Betriebe wesentlich stärker die Gruppe der Haupt- als der Nebenerwerbsbetriebe betrifft. Während in den alten Bundesländern zwischen 
1970-2001 insgesamt ca. 534.000 Haupterwerbsbetriebe die Tätigkeit einstellten, nahm die Zahl der landwirtschaftlichen Betriebe im Nebenerwerb um $158.700 \mathrm{ab}^{7}$. Die Analyse der sozioökonomischen Betriebstypen in den siebziger Jahren kann vereinfachend durch die Untersuchung der damaligen Einkommensstruktur der ländlichen Bevölkerung vorgenommen werden. Die Betrachtung der diesbezüglichen statistischen Daten veranschaulicht eine deutliche Tendenz, in der parallel zu der Abnahme der Anzahl der Betriebe, in denen die Beschäftigung für ihre Inhaber die Haupteinkommensquelle bildete, der Anteil der Betriebe, in denen die Tätigkeit nur ergänzend zum gesamten Einkommen ihrer Inhaber beitrug, wesentlich ansteigt. Der höchste Anteil der Betriebe, in denen das außerbetriebliche Einkommen das betriebliche überstieg, war im Jahr 1970 für Hessen signifikant (ca. $59 \%$ aller Betriebe). Eine große Bedeutung kam den Nebenerwerbsbetrieben auch in Baden - Württemberg (54\%), Rheinland - Pfalz (46\%) und Bayern (41\%) zu. Verhältnismäßig wenig, weil ungefähr nur ein Viertel aller Betriebe, in denen das außerbetriebliche Einkommen das betriebliche überstieg, befanden sich zu dieser Zeit in Schleswig - Holstein (vgl. KRÜLL 1990, S. 31).

Als ein anderes Indiz für die Untersuchung der Änderung der sozialökonomischen Funktion der landwirtschaftlichen Betriebe kann die Verteilung der Arbeitskapazitäten der Mitglieder der Inhaberfamilie der landwirtschaftlichen Betriebe auf die Beschäftigung in und außerhalb des landwirtschaftlichen Betriebes herangezogen werden (vgl. FASTERDing, RIXEN 2005, S. 34 ff.). Der Tabelle 2-7 kann entnommen werden, dass der Anteil der Betriebe mit außerbetrieblich erwerbstätigen Inhabern oder deren Familienangehörigen an der Anzahl aller Betriebe, bis auf die Gruppe von weniger als 5 ha LF, in der Zeitspanne 1983-2001 auf dem früheren Bundesgebiet wesentlich angestiegen ist. Diese Entwicklung ist besonders deutlich im Größenbereich 20-100 ha, wo die angesprochenen Anteile in jeder Betriebsgrößengruppe mehr als verdoppelt wurden. Insgesamt erhöht sich der Anteil der Betriebe mit Erwerbskombinationen im Betrachtungszeitraum von 50,7 \% auf 54,2\%.

\footnotetext{
${ }^{7}$ Es ist an dieser Stelle zu bemerken, dass wegen der Unterschiede der zu bestimmten Zeitpunkten geltenden Abgrenzungsgrundlage zwischen den Neben- und Haupterwerbsbetrieben ein Vergleich hierzu nur bedingt möglich ist. Im Jahr 1970 galten als Nebenerwerbsbetriebe jene Betriebe, in denen der Betriebsinhaber weniger als $50 \%$ seiner Arbeitszeit in seinem Betrieb einsetzte und/oder weniger als $50 \%$ des Gesamteinkommens vom Betriebsinhaberehepaar und Hofnachfolger aus dem landwirtschaftlichen Unternehmen stammten. Seit 1997 erfolgt die Abgrenzung anhand von zwei Kriterien: EGE (Europäische Größeneinheit- 1 EGE entspricht einem Gesamtdeckungsbeitrag von 1.200 EUR) sowie AK (1 AK entspricht einer vollbeschäftigten Person zwischen 18 und 65 Lebensjahr). Demzufolge werden als Nebenerwerbsbetriebe die Betriebe mit 8 bis 16 EGE oder weniger als 1 AK bezeichnet (vgl. BMVEL, diverse Jahrgänge).
} 
Tabelle 2-7: Relative Anteile der Betriebe mit außerbetrieblich erwerbstätigen Inhabern oder ihren Familienangehörigen an der Anzahl aller landwirtschaftlichen Betriebe

\begin{tabular}{|c|c|c|c|c|c|c|}
\hline \multirow[t]{3}{*}{ Betriebsgröße in ha LF } & \multicolumn{6}{|c|}{ Jahr } \\
\hline & 1983 & 1993 & 1995 & 1997 & 1999 & 2001 \\
\hline & \multicolumn{6}{|c|}{ Früheres Bundesgebiet } \\
\hline unter 5 & 66,0 & 63,1 & 62,3 & 59,6 & 63,2 & 62,8 \\
\hline $5-10$ & 75,0 & 77,1 & 63,5 & 75,3 & 75,5 & 76,3 \\
\hline $10-20$ & 48,1 & 63,7 & 64,8 & 66,7 & 68,4 & 69,8 \\
\hline $20-30$ & 23,3 & 42,3 & 44,2 & 44,7 & 48,1 & 50,7 \\
\hline $30-50$ & 13,9 & 27,7 & 30,0 & 30,6 & 33,7 & 36,9 \\
\hline $50-100$ & 10,8 & 20,0 & 20,6 & 19,9 & 20,4 & 22,1 \\
\hline über 100 & 12,5 & 16,2 & 17,4 & 15,6 & 15,4 & 16,5 \\
\hline \multirow[t]{2}{*}{ insgesamt } & 50,7 & 54,4 & 53,9 & 52,0 & 53,4 & 54,2 \\
\hline & \multicolumn{6}{|c|}{ Ost- und Westdeutschland } \\
\hline unter 5 & - & 61,1 & 61,1 & 58,1 & 61,8 & 61,4 \\
\hline $5-10$ & - & 76,6 & 63,5 & 74,7 & 75,1 & 75,9 \\
\hline $10-20$ & - & 63,5 & 64,7 & 66,4 & 68,1 & 69,4 \\
\hline $20-30$ & - & 42,5 & 44,7 & 44,9 & 48,4 & 51,0 \\
\hline $30-50$ & - & 28,0 & 30,5 & 31,0 & 34,1 & 37,2 \\
\hline $50-100$ & - & 20,3 & 21,1 & 20,2 & 20,8 & 22,4 \\
\hline über 100 & - & 14,0 & 16,1 & 12,9 & 13,6 & 14,4 \\
\hline insgesamt & - & 53,6 & 53,1 & 51,1 & 52,4 & 53,2 \\
\hline
\end{tabular}

Quelle: FASTERDING, RIXEN 2005, S. 36

Im Jahr 2003 wurde die Hälfte aller Betriebe in Deutschland im Nebenerwerb bewirtschaftet (siehe Tabelle 2-8). In der Praxis gibt es sowohl solche Nebenerwerbsbetriebe, die als Übergangsstadium von Haupterwerb zu voller Betriebsaufgabe betrachtet werden, als auch solche in denen der Nebenerwerb als Dauereinrichtung anzusehen ist. Die Verbreitung der Nebenerwerbsbetriebe ist nach wie vor bei verschiedenen Betriebsgrößenstrukturen, der Produktionsrichtung, des geltenden Erbrechtes sowie der regional unterschiedlichen Möglichkeiten außerhalb Landwirtschaft ein Einkommen zu erzielen, von Region zu Region unterschiedlich (vgl. DoluschiTZ, SCHWEnNINGER 2003, S.16). Eine besondere Bedeutung besitzen die Nebenerwerbsbetriebe in Mittel- und Süddeutschland. In Ländern wie Baden Württemberg, Hessen und Thüringen übersteigen sie prozentual gemessen die Haupterwerbsbetriebe. Eine relativ große Bedeutung haben die Nebenerwerbsbetriebe auch in den östlichen Bundesländern. Im Jahr 2001 wurden ca. 63 \% aller Betriebe dieses Gebiets im Nebenerwerb geführt. 
Tabelle 2-8: Landwirtschaftliche Betriebe der Rechtsform Einzelunternehmen nach Erwerbsform im Jahr 2003

\begin{tabular}{|c|c|c|c|c|}
\hline Gegenstand der Nachweisung & Einheit & $\begin{array}{l}\text { Haupterwerbs- } \\
\text {-betriebe }\end{array}$ & $\begin{array}{c}\text { Nebenerwerbs- } \\
\text {-betriebe }\end{array}$ & Zusammen \\
\hline \multirow{3}{*}{$\begin{array}{l}\text { Anzahl der Betriebe } \\
\text { LF } \\
\text { Mittlere Flächenausstattung }\end{array}$} & \multicolumn{4}{|c|}{ Deutschland } \\
\hline & $\begin{array}{l}\text { in } 1000 \\
\text { in } 1000 \text { ha } \\
\text { in ha }\end{array}$ & $\begin{array}{c}176,4 \\
9044,5 \\
51,3\end{array}$ & $\begin{array}{c}212,4 \\
2655,6 \\
12,5\end{array}$ & $\begin{array}{c}388,7 \\
11700,1 \\
30,1 \\
\end{array}$ \\
\hline & \multicolumn{4}{|c|}{ Früheres Bundesgebiet } \\
\hline $\begin{array}{l}\text { Anzahl der Betriebe } \\
\text { LF } \\
\text { Mittlere Flächenausstattung }\end{array}$ & $\begin{array}{l}\text { in } 1000 \\
\text { in1000 ha } \\
\text { in ha }\end{array}$ & $\begin{array}{c}167,9 \\
7911,9 \\
47,1 \\
\end{array}$ & $\begin{array}{c}197,4 \\
2399,6 \\
12,2 \\
\end{array}$ & $\begin{array}{c}365,4 \\
10311,5 \\
28,2 \\
\end{array}$ \\
\hline & \multicolumn{4}{|c|}{ Neue Länder } \\
\hline $\begin{array}{l}\text { Anzahl der Betriebe } \\
\text { LF } \\
\text { Mittlere Flächenausstattung }\end{array}$ & $\begin{array}{l}\text { in } 1000 \\
\text { in } 1000 \text { ha } \\
\text { in ha }\end{array}$ & $\begin{array}{c}8,4 \\
1132,7 \\
134,2 \\
\end{array}$ & $\begin{array}{c}14,9 \\
255,9 \\
17,2 \\
\end{array}$ & $\begin{array}{c}23,3 \\
1388,6 \\
59,5\end{array}$ \\
\hline
\end{tabular}

In der Aufteilung der landwirtschaftlichen Betriebe nach Rechtsformen bilden mit deutlichem Abstand die Einzelunternehmen der natürlichen Personen die zahlreichste Gruppe. Die statistischen Daten aus den letzten Jahren deuten auf einen Trend hin. Während die Anzahl der landwirtschaftlichen Betriebe in Form der Einzelunternehmen abnimmt, vergrößert sich ständig die Anzahl der Betriebe in Form der Personengesellschaften. Diese Entwicklung weist einen bedeutend schnelleren Verlauf in Westdeutschland als in den östlichen Bundesländern auf. Dies schlägt sich auch in der Änderung der relativen Anteile der Betriebe bestimmter Rechtsformen an der gesamten LF nieder. Eine rückläufige Tendenz ist auch im Fall der juristischen Personen zu verzeichnen. So nimmt die Anzahl der landwirtschaftlichen Betriebe dieser Rechtsform zwischen 1995 und 2003 um rund 40\% ab. Der Anteil dieser Betriebsgruppe an der gesamten LF nimmt, in der angesprochener Zeitspanne, von 58,5\% auf ca. $53 \%$ ab (siehe Tabelle 2-9). 
Tabelle 2-9: Landwirtschaftliche Betriebe nach Rechtsformen

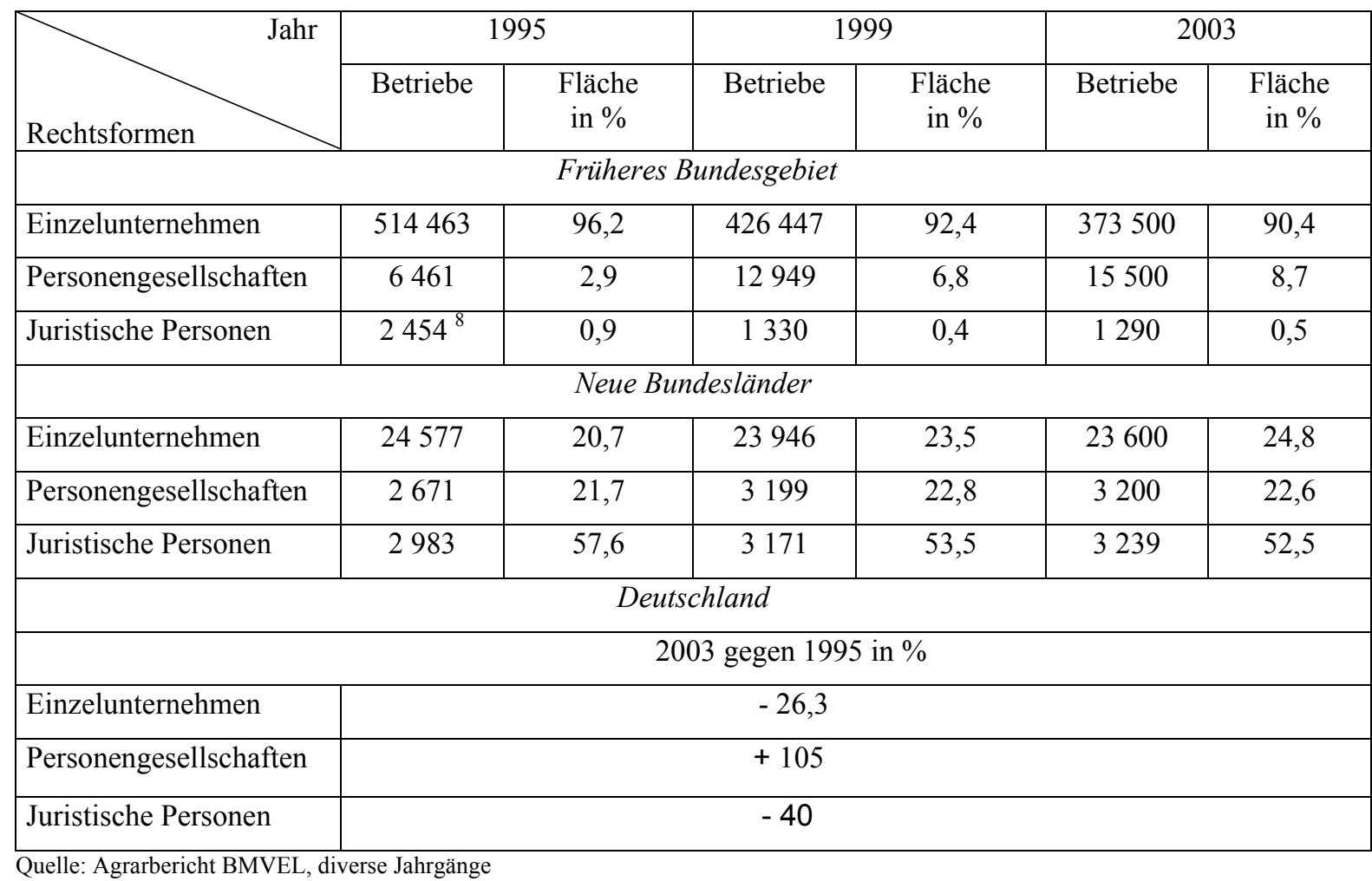

\subsection{Technische Ausstattung}

In der deutschen Landwirtschaft, bezogen auf das frühere Bundesgebiet, wurde in den letzten Jahrzehnten eine einschneidende Modernisierung der Mechanisierung vollzogen. Signifikant für diesen Vorgang ist eine Zunahme der Motorleistung der Schlepper je 100 ha LF. In einer relativ kurzen Zeitspanne 1970-1985 wird die Zahl, der pro 100 ha LF entfallenden $\mathrm{kW}$ fast verdoppelt. Während im Jahr 1970 einer Einheit von 100 ha LF die Motorleistung von ca. 206 kW gegenüberstand, nahm diese Zahl im Jahr 1985 auf rund $395 \mathrm{~kW}$ zu (siehe Abbildung 2-6).

Diese Tendenz wird nicht durch die Steigerung der Anzahl einzelner Schlepper vollzogen. Die deutliche Zunahme der motorischen Zugkraft pro Flächeneinheit wird im Wesentlichen durch die Steigerung der Motorleistung der einzelnen Schlepper herbeigeführt. Während die Gesamtzahl der Schlepper in der angesprochenen Zeitspanne um 1,5 \% zunimmt (1970:12341985:1253), steigt die Motorleistung eines Schleppers von 20,6 kW auf rund $32 \mathrm{~kW}$ an.

\footnotetext{
${ }^{8}$ Einschließlich LPG in Liquidation.
} 
Abbildung 2-6: Die Entwicklung der Motorleistung der Schlepper in den landwirtschaftlichen Betrieben des früheren Bundesgebiets in den Jahren 1970-1995

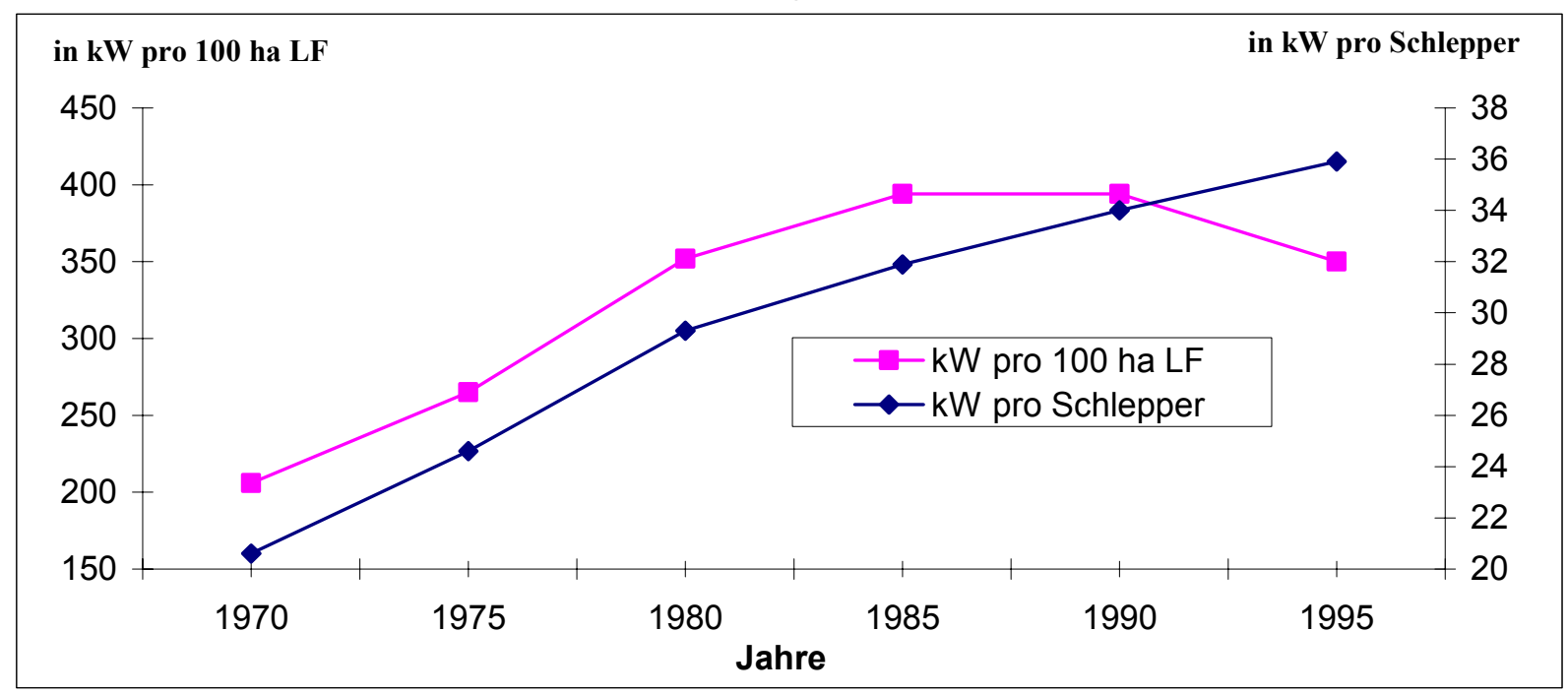

Quelle: Statistisches Bundesamt, diverse Jahrgänge

Bis Anfang der neunziger Jahre erhöhte sich die Motorleistung der Schlepper pro 100 ha LF konstant. Danach setzte eine gegenläufige Entwicklung ein. Während im Jahr 1988 pro 100 ha LF noch annähernd $400 \mathrm{~kW}$ Motorleistung entfielen, wurde die Zahl bis zum Jahr 1995 auf ca. 340 kW reduziert. Der angesprochene Rückgang der Zahl der kW pro 100 ha LF geht mit einer kontinuierlichen Steigerung der Motorleistung der einzelnen Schlepper einher. So stieg die durchschnittliche Motorleistung eines Schleppers bis zum Jahr 1996 auf ca. 37 $\mathrm{kW}$ an.

Im Jahr 1995 gab es in den landwirtschaftlichen Betrieben des früheren Bundesgebiets über 1,07 Mio. Schlepper. Die zahlreichste Gruppe bildeten die Schlepper mit einer Motorleistung von 25-40 kW. An der zweiten Stelle rangierten die Schlepper mit einer Motorleistung von 40-60 kW. Dementsprechend beliefen sich die Anteile der Schlepper beider Gruppen an gesamter Schlepperanzahl auf jeweils $35 \%$ bzw. $31 \%$. Relativ stark waren auch die Schlepper mit einer Motorleistung unter $25 \mathrm{~kW}$ vertreten. Auf diese Gruppe entfielen ungefähr $19 \%$ aller Schlepper. Lediglich ca. 5\% aller Schlepper zählte zu der Gruppe mit einer Motorleistung von über $60 \mathrm{~kW}$ (siehe Tabelle 2-10).

\footnotetext{
9 Im Jahr 1995 wurde eine detaillierte Auswertung der statistischen Daten zur Mechanisierung der landwirtschaftlichen Betriebe mit der Aufteilung nach Bundesländern und Motorleistung der Maschinen zum letzten Mal durchgeführt und bis zum Jahr 2005 nicht wieder aufgenommen. Anfang des Jahres 2006 wurde erneut eine empirische Untersuchung zur Mechanisierung der Landwirtschaft unternommen. Zum Zeitpunkt der Fertigstellung dieser Arbeit befanden sich die Daten in Auswertungsphase und standen nicht zur Verfügung (Zepuntke 2006). Für eine allgemeine Analyse des aktuellen Zustandes der Mechanisierung der deutschen Landwirtschaft wird auf die Tabellen A.1 und A.2 im Anhang verwiesen.
} 
Tabelle 2-10: Struktur des Schlepperbestandes in den landwirtschaftlichen Betrieben des früheren Bundesgebiets im Jahr 1995

\begin{tabular}{|c|c|c|c|c|c|c|}
\hline \multirow{2}{*}{$\begin{array}{c}\text { Betriebsgröße } \\
\text { in ha LF }\end{array}$} & \multirow{2}{*}{$\begin{array}{c}\text { Anzahl } \\
\text { der } \\
\text { Schlepper }\end{array}$} & \multicolumn{5}{|c|}{ Schlepper mit Motorleistung in kW (PS) } \\
\hline & & $\begin{array}{c}\text { unter } 25 \\
\text { (unter } 34 \text { PS ) }\end{array}$ & $\begin{array}{c}25-40 \\
(34-54 \text { PS) }\end{array}$ & $\begin{array}{c}40-60 \\
\text { (54-82 PS) }\end{array}$ & $\begin{array}{c}60-75 \\
(82-102 \mathrm{PS})\end{array}$ & $\begin{array}{c}60-75 \\
(82-102 \mathrm{PS})\end{array}$ \\
\hline unter 5 & 167680 & 71300 & 76200 & 18290 & 1530 & 360 \\
\hline $5-10$ & 138980 & 36610 & 68400 & 30940 & 2590 & 440 \\
\hline $10-20$ & 216020 & 37370 & 88790 & 76510 & 11320 & 2030 \\
\hline $20-50$ & 352590 & 39960 & 104920 & 140510 & 52010 & 15190 \\
\hline $50-100$ & 152750 & 10830 & 30680 & 55390 & 33280 & 22570 \\
\hline 100 und mehr & 45940 & 2130 & 6150 & 12900 & 10340 & 14420 \\
\hline zusammen & 1073960 & 198200 & 375140 & 334540 & 111070 & 55010 \\
\hline in $\%$ & 100 & 19 & 35 & 31 & 10 & 5 \\
\hline
\end{tabular}

Quelle: Statistisches Bundesamt 1995

Die vorher geschilderten regionalen Unterschiede in den Betriebsgrößenstrukturen spiegeln sich auch in der technischen Ausstattung wider. Gemessen an der pro 100 ha LF entfallenden Motorleistung, am stärksten mechanisiert waren im Jahr 1995 die landwirtschaftlichen Betriebe in den südlichen Regionen, allen voran in Bayern. Dementsprechend war die Motorleistung je 100 ha LF in diesem Bundesland um das Zweifache höher als in Schleswig Holstein (siehe Tabelle 2-11).

Tabelle 2-11: Motorleistung der Schlepper pro eine Flächeneinheit von 100 ha LF in ausgewählten Ländern des früheren Bundesgebietes im Jahr 1995

\begin{tabular}{|l|c|c|c|c|c|}
\hline & Bayern & $\begin{array}{c}\text { Baden- } \\
\text { Württemberg }\end{array}$ & $\begin{array}{c}\text { Nordrhein- } \\
\text { Westfalen }\end{array}$ & Niedersachsen & $\begin{array}{c}\text { Schleswig- } \\
\text { Holstein }\end{array}$ \\
\hline Motorleistung & 476 & 443 & 343 & 273 & 236 \\
\hline in kW pro 100 ha LF & 476 & & \\
\hline
\end{tabular}

\subsection{Direkte und indirekte Förderung des Modernisierungsprozesses}

Einen vielfältigen Einfluss auf den geschilderten Strukturwandel in der deutschen Landwirtschaft hatte die staatliche Förderungspolitik. Dies gilt besonders für die Finanzierungshilfen für die Investitionen in den landwirtschaftlichen Betrieben. Die wichtigsten Instrumente der direkten Förderung sind das einzelbetriebliche Investitionsförderungsprogramm (EFP) und das Agrarkreditprogramm (AKP). Bei indirekter Förderung handelt es sich um einkommensteuerwirksame Sonder- und Ansparabschreibungen. Im Folgenden werden diese Instrumente in einem für die Überlegungen dieser Arbeit relevanten Rahmen komprimiert dargestellt. 


\subsubsection{Förderung der einzelbetrieblichen Investitionen}

Die Grundsätze der Maßnahmen für die investive Förderung wurden im Gesetz über die Gemeinschaftsaufgabe zur Verbesserung der Agrarstruktur und des Küstenschützes (GAK) festgelegt (vgl. BGBI. I 1969, S. 1573; BGBI. I 1971, S. 2140). Dieses Programm, das von Bund und Ländern gemeinsam koordiniert wurde, umfasste mehrere zum Teil unterschiedlich konzipierte Investitionsförderungsmaßnahmen. Von besonderer Bedeutung für die Modernisierung der Mechanisierung war die Förderung der Investitionen in einzelnen Betrieben und Kooperationen. Die Förderung im Rahmen dieses Programms hatte zum Ziel, den Inhabern von langfristig entwicklungsfähigen Betrieben eine Hilfe für die Verbesserung der Allokation der Produktionsfaktoren, damit für Wachstumserleichterung und Einkommensverbesserung anzubieten. Die Zuwendungen für die Investitionen wurden in Form von Zinsverbilligungen für Kapitalmarktdarlehen und verlorenen Zuschüssen gewährt. Im Fall der zinsverbilligten Kredite wurden die oberen und unteren Grenzen der Investitionsvolumen festgelegt. Am Beginn des Förderungsprogramms lag das minimale Investitionsvolumen bei $20.000 \mathrm{DM}$ und das maximale bei $600.000 \mathrm{DM}$ pro Unternehmen. Dabei durfte die förderungsfähige Investition den Betrag von 145.000 DM pro Vollarbeitskraft nicht übersteigen. Die Zinsverbilligung, die bis zu $5 \%$ reichte, konnte bis zur Gesamthöhe des aufgenommenen Kredits gewährt werden. Die Laufzeit der zinsverbilligten Kredite wurde dem Investitionsgegenstand angepasst und betrug bei Immobilien bis zu 20 Jahren und bei anderen Investitionsobjekten bis zu 10 Jahren. Den einzelnen Ländern stand das Recht zu, statt die Zinsverbilligung in kapitalisierter Form einen Zuschuss für die Investitionen zu gewähren.

Bei der Förderung wurde eine Selektion von Betrieben in entwicklungsfähige und nicht entwicklungsfähige vorgenommen. Eine Sonderstellung in dem Förderungsprogramm genossen die landwirtschaftlichen Futterbau- und Grünlandbetriebe der benachteiligten Regionen. Auf diese Weise wurde der Versuch unternommen die natürlichen Wettbewerbsnachteile bestimmter Regionen zumindest teilweise zu kompensieren. Die Hilfe des Förderungsprogramms konnte nur den Antragstellern gewährt werden, die entweder eine landwirtschaftliche Ausbildung oder eine mindestens dreijährige Erfahrung im landwirtschaftlichen Beruf nachweisen konnten. Die Förderung setzte auch die Einführung einer ordnungsmäßigen Buchführung für wenigstens 10 Jahre, beginnend im Jahr der Inanspruchnahme der Fördemittel voraus. Als Maßstab für die Beurteilung der Entwicklungsfähigkeit der landwirtschaftlichen Betriebe galt das Arbeitseinkommen je eine 
AK. So mussten die Antragsteller anhand der vorgelegten Entwicklungspläne nachweisen, dass die geplante Investition spätestens im vierten Jahr nach der Inanspruchnahme der Förderung ein mit anderen Wirtschaftssektoren vergleichbares Einkommensniveau pro AK zu erreichen hilft. Es war das Ziel dieser Anordnung, die Anreize für die Fortsetzung der Betriebsführung $\mathrm{zu}$ schaffen. Das durchschnittliche Einkommen wurde unter Berücksichtigung der regionalen Wirtschaftsbedingungen unterschiedlich festgesetzt. Innerhalb des gesamten Bundesgebietes wurden 27 Regionen gegliedert. Die Abgrenzung erfolgte regional uneinheitlich. Während es in Bayern zehn Regionen gab, wurde Niedersachsen als eine Einheit betrachtet. Anfang der achtziger Jahre wurde zusätzlich die sog. Prosperitätsklausel konkretisiert. Sie besagte, dass eine Investition in Betrieben, in denen das Arbeitseinkommen bereits zum Zeitpunkt der Antragstellung das festgelegte durchschnittliche Einkommen um $20 \%$ und mehr überstieg, nicht gefördert werden konnten. Das Abgrenzungskriterium der regional differenzierten Einkommenshöhe wurde mehrmals kontrovers diskutiert. Dabei wurde besonders häufig das Argument vorgetragen, dass diese Bedingung nicht mit der allgemeinen wirtschafts- und agrarpolitischen Zielsetzung, die Einkommensdifferenzen zwischen einzelnen Regionen $\mathrm{zu}$ nivellieren, konform war (vgl. BLOCK 1977, S. 210 ff.). Das Kriterium des durchschnittlichen Einkommens wurde nach zahlreichen Modifizierungen Anfang achtziger Jahre abgeschafft.

Eine wichtige Stellung in dem geschilderten Programm nahm auch die Förderung von Kooperationen ein. Gefördert wurden Investitionen in Kooperationsformen, die auf die Flächenbewirtschaftung gerichtet waren. Hierzu zählte auch die Zusammenarbeit im Rahmen der Maschinenringe, sofern die vorgenommenen Investitionen dem Interesse dieser Kooperationsform dienten. Die Förderung der einzelnen Antragsteller richtete sich nach den Grundsätzen für die Förderung der Einzelbetriebe. Darüber hinaus umfasste dieses Programm auch Zuschüsse für den organisatorischen Aufwand der Kooperationen. Die Zuschüsse wurden in den ersten drei Jahren nach der Gründung der Kooperation gezahlt und reichten von $60 \%$ im ersten Jahr bis zu $20 \%$ im dritten Jahr der anfallenden Gründungs- und Verwaltungskosten (vgl. BML 1974, S. 30 ff.).

Neben dem EFP wurde im Jahr 1984 neu das Agrarinvestitionsförderungsprogramm eingeführt. Gefördert wurden hier neben den Haupt- auch die Nebenerwerbsbetriebe. Als förderungsfähig wurden Investitionen angesehen, die der Verwirklichung folgender Ziele dienten: 
- Anpassung der Betriebsstruktur an die veränderten Marktbedürfnisse,

- Senkung der Produktionskosten und Verbesserung der Arbeitsbedingungen,

- Energieeinsparung,

- Verbesserung des Umweltschutzes.

Die Förderung erfolgte in Form einer Zinsverbilligung für Kapitalmarktdarlehen. Die Maschineninvestitionen konnten bis zu $40 \%$ des Anschaffungspreises bezuschusst werden. Dabei mussten die baren Eigenleistungen mindestens $60 \%$ betragen. Unter bestimmten Bedingungen konnten von den in einzelnen Regionen für die Förderungsvergabe zuständigen Behörden die erforderlichen Anteile des Eigenkapitals auf $40 \%$ gesenkt werden. Die Anschaffungspreise der förderungsfähigen Maschinen durften nicht über 40.000 DM pro Betrieb hinausgehen. Die Zinsverbilligung reichte bis $\mathrm{zu} 3 \%$ in nicht benachteiligten Gebieten und bis $\mathrm{zu} 5 \%$ in benachteiligten Gebieten. Die Zinsverbilligung wurde nach Abzinsung als einmaliger Zinszuschuss ausgezahlt, nachdem die Aufnahme des Darlehens und die Durchführung der Investitionen nachgewiesen worden waren (vgl. BML 1987, S. 30 ff.).

Die Förderung der investiven Maßnahmen im Rahmen der GAK wird auch heute fortgeführt. Das zentrale Förderungsinstrument der einzelbetrieblichen Investitionen ist das Agrarinvestitionsförderungsprogramm. Im Rahmen dieses Programms wird nach wie vor eine Investitionshilfe in Form von Zinsverbilligung für Kapitalmarktdarlehen und Zuschüssen gewährt. Die Zinsverbilligung kann bis zu $5 \%$ des Kapitalmarktdarlehens reichen. Eine besondere Bedeutung kommt hier der Förderung der Investitionen in dem Umweltschutz (Emissionsminderung, Energieeinsparung), dem ökologischen Landbau und den tiergerechten Haltungsverfahren zu. Nicht mehr förderungsfähig sind grundsätzlich die Investitionen in Maschinen für die Außenwirtschaft. Eine Ausnahme gilt für Maschinen und Geräte für eine besonders umweltgerechte Ausrichtung der Produktion und für nachwachsende Rohstoffe. Dementsprechend gefördert werden Anschaffungen von:

- $\quad$ Pflanzenschutzgeräten, die mit anerkannten technischen Eichrichtungen ausgestattet sind,

- Geräten zur bodennahen Flüssigmistausbringungs- und direkten -einarbeitungstechnik, Geräten für bodenschonende Bearbeitungs- und Bestellungstechnik, 
- Empfangsgeräten und Software von globalen Positionierungssystemen (GPS) zur

Erfassung von Erntemengen, Boden- und Pflanzeneigenschaften bei der teilflächenspezifischen Bewirtschaftung (vgl. BMELV 2006) ${ }^{10}$.

\subsubsection{Einkommensteuerwirksame Förderung}

Im Gegenteil zur Methodik der Absetzung für Abnutzung (AfA) für Gebäude sind in Deutschland für bewegliche Wirtschaftsgüter (WG) keine Abschreibungsdauer und kein -satz gesetzlich vorgeschrieben. Das Finanzministerium gibt auf der Grundlage der bisherigen Erfahrungen der steuerlichen Praxis eine AfA- Tabelle, die die in etwa einzuhaltenden AfAZeiten enthält. Abweichungen von den vorgegebenen Werten sind soweit der Betroffene dies begründen kann, zulässig. Im Einkommensteuergesetz werden drei Arten von AfA unterschieden:

- nach der betriebsgewöhnlichen Nutzungsdauer (ND),

- nach der Maßgabe der Leistung,

- für außergewöhnliche wirtschaftliche und technische Abnutzung (AfaA).

Eine Besonderheit im deutschen EStG betrifft die so genannte Sonder-AfA. Laut ursprünglicher Regelung konnte in landwirtschaftlichen Betrieben - neben der linearen AfA im ersten und in den zwei nach der Anschaffung einer Maschine folgenden Jahren eine Sonderabschreibung in Höhe von 50 \% der Maschinenanschaffungskosten vorgenommen werden. Die Wirkung dieser Abschreibungsmethodik erstreckte sich im Wesentlichen auf:

- die Gewinnglättung und damit Milderung der Steuerprogression,

- den Steueraufschub und Zinsgewinn,

- die zeitweise Gewinnsenkung für die Inanspruchnahme von öffentlichen Vergünstigungen wie z.B. Steuerermäßigungsbetrag, Freibetrag nach $\S 14$ a Abs.4 EStG, Wohnungsbauprämie oder Bafög für Kinder (vgl. KöHNE, Wesche 1982, S. 235).

Die Sonder- AfA in ursprünglicher Form barg die Gefahr, zu Fehlinvestitionen zu verleiten. Dabei handelt es sich um die Vornahme von Investitionen, die aus wirtschaftlicher Sicht nicht

\footnotetext{
${ }^{10}$ Die im Rahmen der Gemeinschaftsaufgabe realisierte Förderung der Investitionen in die oben genannte Technik wird mit Ende des Jahres 2006 beendet. Es bedeutet, dass ab 2007 keine Investitionen in die Maschinen für die Außenwirtschaft in der hier genannten Form bezuschusst werden. Die Förderung der Maschineninvestitionen kann weiterhin auf der Ebene der einzelnen Bundesländer im Rahmen von länderspezifischen Förderprogrammen erfolgen (Rosenauer 2006).
} 
gerechtfertigt werden konnten und trotzdem für das Erreichen der oben angesprochenen Vergünstigungen realisiert wurden. Die negativen Erfahrungen aus der Praxis führten dazu, dass die Sonder- AfA im Laufe der Zeit substantiell modifiziert wurde. Heute ist sie nur den kleineren und mittleren Betrieben vorbehalten. Dabei handelt es sich um Betriebe, deren Einheitswert zum Zeitpunkt der Anschaffung des Wirtschaftsgutes, für das die Sonder- AfA in Anspruch genommen wird, nicht über 122.710 EUR hinausgehen soll $(\S 7 \mathrm{~g} \mathrm{EStG})^{11}$. Die Sonder- AfA darf insgesamt nicht über $20 \%$ der Anschaffungskosten im Jahr der Investitionsvornahme und in den vier folgenden Jahren hinausgehen. Die Sonder- AfA ist nur dann erlaubt, wenn vorher für die Investitionsgüter eine Ansparrücklage gebildet wurde. Gemäß $§ 7 \mathrm{~g}$ Abs. 3 EStG darf die Rücklage 40 \% der Anschaffungs- oder Herstellungskosten des begünstigten Wirtschaftsguts nicht überschreiten, das voraussichtlich bis zum Ende des zweiten auf die Bildung der Rücklage folgenden Wirtschaftsjahres angeschafft oder hergestellt wird. Sobald das WG, für das die Rücklage vorgenommen wurde, angeschafft wird, ist sie in voller Höhe, d.h. bis zu $40 \%$ der Anschaffungskosten gewinnerhöhend aufzulösen. Im Fall, in dem die Rücklage am Ende des zweiten Wirtschaftsjahres noch vorhanden ist, d.h. die geplante Investition nicht vorgenommen wurde, tritt die Konsequenz ein, dass die Rücklage für jedes Wirtschaftsjahr, in dem sie bestanden hat, um $6 \%$ des gebildeten Rücklagebetrages zu erhöhen ist.

Die Wahrnehmung der Sonder- AfA kann von Betrieb zu Betrieb unterschiedliche Bedeutung haben. Eine Analyse der Wirkung dieser Abschreibungsmethodik bedarf einer fallspezifischen Betrachtung. Vorbehaltlich dessen wird in Tabelle 2-12 eine Leitlinie bei der Beurteilung des Einflusses der aktuell geltenden Sonder- AfA auf die zeitliche Verteilung der Steuerlast und bei dem damit verbunden Zinsgewinn bei Abschreibung eines exemplarischen Wirtschaftsgutes aufgezeigt. Im ersten Teil dieser Tabelle werden die einzelnen Posten bei der Inanspruchnahme der linearen AfA dargestellt. Es wird hier ebenso wie im weiteren Teil der Kalkulation einheitlich ein Steuersatz von $25 \%$ unterstellt. In der mittleren Zeile der Tabelle wird neben der Normalabschreibung auch die Sonder- AfA und zwar in den ersten zwei Jahren je 4.000 EUR vorgenommen. Dies führt zu einer Verminderung des zu versteuernden Einkommens, folglich $\mathrm{zu}$ einer Steuerersparnis von 1.000 EUR pro Jahr. In den darauf folgenden Jahren wird das zu versteuernde Einkommen wieder angehoben. Dies ist darauf zurückzuführen, dass nach der Wahrnehmung der Sonder- AfA nur noch ein geringeres

\footnotetext{
${ }^{11}$ Als Einheitswert wird der Wert des luf Vermögens, des Grundvermögens und des Betriebsvermögens des Steuerpflichtigen bezeichnet ( $33 \mathrm{f}$. BewG). Dieser Wert wird in erster Linie als Grundlage für die Ermittlung der Bemessungsgrundlage für die Grundsteuer, die Vermögensteuer, die Erbschaftsteuer und die Grunderwerbsteuer herangezogen.
} 
Abschreibungsvolumen zur Verfügung bleibt. Die geringen Abschreibungen vom dritten Jahr bis Ende der ND im Fall der Sonder- AfA haben zur Folge, dass der ursprüngliche Vorsprung nivelliert und die Steuerlast in beiden Varianten ausgeglichen wird. Dieses Ergebnis wird umgewandelt, wenn die zeitliche Verschiebung der Steuerlast und die damit verbunden Zinsvorteile berücksichtigt werden. Unter Einbeziehung eines Zinsvorteils von $6 \%$ beträgt die Ersparnis bei Inanspruchnahme der Sonder- AfA in dem dargestellten Beispiel insgesamt 530 EUR.

Tabelle 2-12: Beispielsdaten zur Wirkung der Sonderabschreibung bei konstanten Gewinnen

\begin{tabular}{|c|c|c|c|c|}
\hline Jahr & I & II & III-X & $\sum \mathrm{I}-\mathrm{X}$ \\
\hline Zu versteuerndes Einkommen & 50000 & 50000 & 50000 & 500000 \\
\hline Anschaffungskosten & 40000 & - & - & 40000 \\
\hline $\mathrm{Zu}$ versteuerndes Einkommen & & & & \\
\hline bei linearer- AfA (von $10 \%$ pro Jahr) & 46000 & 46000 & 46.000 & 460000 \\
\hline fällige Steuer & 11500 & 11500 & 11500 & 115000 \\
\hline Sonder- AfA & 4000 & 4000 & - & - \\
\hline $\mathrm{Zu}$ versteuerndes Einkommen & & & & \\
\hline bei Sonder- AfA & 42000 & 42000 & 47000 & 460000 \\
\hline fällige Steuer & 10500 & 10500 & 11750 & 115000 \\
\hline Steuerersparnis & 1000 & 1000 & -250 & - \\
\hline Gegenwartswert der Steuerersparnis & 943 & 890 & -1303 & 530 \\
\hline
\end{tabular}

Diese Überlegungen können um weitere Aspekte erweitert werden. Bei der Kombination der Sonder- AfA mit der degressiven Abschreibungsmethodik können die Abschreibungen noch stärker vorgezogen werden. Dies ist besonders vorteilhaft, wenn ein relativ gutes Wirtschaftsjahr vorliegt und/oder in den nächsten Jahren weitere aufwendige Investitionen geplant sind. Neben dem erläuterten Zinsvorteil ist die Sonder- AfA besonders vorteilhaft, wenn größere Gewinnschwankungen bestehen. Dann können diese geglättet werden, was bei progressivem Tarif zu einer Steuerminderung führt.

\subsection{Zusammenfassung}

In der westdeutschen Landwirtschaft wurde in den letzten Jahrzehnten ein tiefgreifender Strukturwandel vollzogen. Seit dem Jahr 1970 wurde die Anzahl aller landwirtschaftlichen Betriebe ungefähr um den Faktor drei verkleinert. Die Gruppe aller in der Landwirtschaft tätigen Personen ist im gleichen Zeitraum um mehr als die Hälfte vermindert.

Trotz dem ständigen Trend zur wenigen und größeren Einheiten herrschen in den alten Bundesländern auch heutzutage kleine Betriebe vor. Wesentlichen Änderungen unterliegt die soziökonomische Funktion der landwirtschaftlichen Betriebe. Dies spiegelt sich in einer stetigen Zunahme der Anteile der Nebenerwerbsbetriebe wider. 
Für viele kleine Betriebe ist eine effiziente Verwendung der vorhandenen bzw. zugänglichen Faktoren in einzelbetrieblicher Lösung nicht möglich. Unter diesen Bedingungen kommt eine wesentliche Bedeutung der überbetrieblichen Zusammenarbeit zu. Denn auf diese Weise werden Voraussetzungen geschaffen zum einen die Nachteile der ungünstigen Strukturen zu mindern und zum anderen die Möglichkeiten weiter Entwicklung zu vergrößern. Die Steigerung der Bedeutung der überbetrieblichen Zusammenarbeit ist nicht zuletzt an der Zunahme der Betriebe, die in Rechtsform der Personengesellschaften geführt werden, erkennbar.

Schließlich zeigen die Erfahrung aus der Praxis, dass die staatliche Förderung von Maschineninvestitionen, sei es in direkter oder indirekter Form, die Gefahr birgt zu Maschinenanschaffungen $\mathrm{zu}$ verleiten, die aus wirtschaftlicher Sicht nicht gerechtfertigt werden können. Die falsch gesetzten Signale können unter bestimmten Bedingungen zur Übermechanisierung führen, die statt $\mathrm{zu}$ fördern sich negativ auf die Betriebsentwicklung auswirken. 


\section{Entwicklung, Formen, Erfolge und Probleme der kooperativen Maschinennutzung in Deutschland}

Nach der vorangegangenen kurzen Beschreibung des deutschen Agrarsektors werden im Folgenden die Ergebnisse der Untersuchung zur überbetrieblichen Zusammenarbeit beim Maschineneinsatz dargestellt. Dies geschieht v.a. in der Absicht daraus Schlussfolgerungen für mögliche Kooperationsformen in Polen abzuleiten.

Die Zusammenarbeit im Maschineneinsatz bildet lediglich einen Teil aller in der deutschen Landwirtschaft vorhandenen Kooperationsformen. Um eine systematische Abgrenzung der Formen der Zusammenarbeit im Bereich der Maschinennutzung vornehmen zu können, werden zunächst alle in der Praxis relevanten Kooperationsformen komprimiert dargestellt. Diese Aufgabe erfolgt anhand einer Auswertung der einschlägigen Literatur. In diesem Zusammenhang werden allgemeine Begriffe und Ziele sowie der unterschiedliche Bindungsgrad der Kooperationsformen erläutert. Alsdann wird allgemein der Rechtsrahmen der überbetrieblichen Maschinennutzung kurz dargestellt. Im weiteren Teil dieses Kapitels wird das Augenmerk punktuell auf die dem Thema der Arbeit immanenten Formen der Zusammenarbeit von der Nachbarschaftshilfe bis hin zur Auslagerung der Maschinenarbeit an Lohnunternehmen (LU) gerichtet.

\subsection{Begriff und Ziele}

Unter dem Begriff Kooperation wird im engeren Sinn eine freiwillige, vertraglich (mündlich oder schriftlich) vereinbarte Zusammenarbeit selbständig wirtschaftender Unternehmen verstanden. Kennzeichnend für die Zusammenarbeit ist, dass sie nicht administrativ verordnet ist, auf Eigeninitiative beruht, auf längere Dauer angelegt ist und sich nicht auf eine einmalige oder sporadische Geschäftsabwicklung beschränkt (vgl. DoLUSCHITZ 2001, S.375).

Im Bereich der Landwirtschaft unterscheidet man grundsätzlich zwischen zwei Typen der Kooperation. Die erste Form, die horizontale Kooperation, betrifft die Relationen zwischen einzelnen Landwirten, die meistens gleichartige oder ähnliche Güter anbieten. Die zweite Form dagegen, die als vertikale Kooperation bezeichnet wird, umfasst die Zusammenarbeit zwischen bestimmten landwirtschaftlichen Betrieben und Betrieben der vor- und nachgelagerten Bereiche, wie z.B. einem Milchkuhhaltungsbetrieb und einer Molkerei (vgl. GABLERS WIRTSCHAFTLEXIKON 1980, S.2420,THEUVSEN 2003, S.4). 
Die allgemeinen Ziele der Kooperation im Bereich Landwirtschaft lassen sich grundsätzlich in zwei Gruppen einteilen (vgl. z.B. LINK 1995, S.40, DoLusCHITZ 2001, S.375). Zu der ersten Gruppe, die aus den auf die Verbesserung der Einkommenssituation einzelner Betriebe gerichteten Faktoren besteht, zählen vor allem:

- Kostensenkung, die z.B. auf dem Weg der Nutzungsrationalisierung und Verbesserung der Auslastung von vorhandenen oder $\mathrm{zu}$ beschaffenden Einrichtungen (Maschinen, Anlagen) erzielt wird,

- $\quad$ Leistungssteigerung durch den Einsatz moderner Verfahren,

- $\quad$ Senkung des Kapitalbedarfs für Investitionen,

- Verbesserung der Vertrauenswürdigkeit bei Kreditaufnahme, Steigerung der Investitionsmöglichkeiten,

- $\quad$ Erleichterung durch eine bessere Maschinenauslastung und einen rechtzeitigen Maschinenwechsel der Teilnahme am technischen Fortschritt,

- $\quad$ Freisetzung von Kapazitäten, besonders in Form von Arbeit und Kapital, die für eine weitere Betriebsentwicklung oder das Nachgehen einer einkommenswirksamen Beschäftigung außerhalb der Landwirtschaft verwendet werden können,

- Stärkung der Position einzelner Betriebe beim gemeinsamen Absatz der Erzeugnisse,

- $\quad$ Sicherung und Verbilligung des Bezugs von Produktionsmitteln,

- $\quad$ Funktionsteilung zwischen den zusammenarbeitenden Landwirten,

- $\quad$ Stabilisierung und Sicherung der Unternehmensführung durch Bindung und Nutzen von Wissen der Kooperationspartner,

- $\quad$ Minderung des Risikos von Fehlinvestitionen.

Zu der zweiten Gruppe, die die sozialen Ziele umfasst, gehören:

- Arbeitserleichterung, Verringerung der Aufgabenvielfalt und damit der psychischen und physischen Belastung der im landwirtschaftlichen Betrieb beschäftigten Personen,

- Zeitersparnisse, die für weitere berufliche Spezialisierung oder Erholung in Anspruch genommen werden können,

- Entlastung von stark beanspruchten Frauen, Kindern oder sonstigen Familienangehörigen, 
- $\quad$ Sicherung der Kontinuität des Betriebsablaufs im Fall von Urlaub, Krankheit oder Tod des Betriebsleiters.

Den skizzierten Zielen kommt bei der Gestaltung gegenseitiger Beziehungen eine unterschiedlich große Bedeutung zu. Welche Beweggründe und Ziele für die Anknüpfung und Fortsetzung der Kooperationsbeziehungen den Ausschlag geben, hängt vom Einzelfall ab (vgl. z.B. ManN, Muziol 2001, S. 55-97). Das Spektrum von Vorteilen, die hier realisiert werden, nimmt i.d.R. parallel zu der Steigerung des Umfangs und der Bindungsintensität der Zusammenarbeit zu.

\subsection{Praxisrelevante Kooperationsformen und ihre Bindungsintensität}

Bezüglich der Systematik der in der Praxis vertretenen Kooperationsformen bietet die Fachliteratur grundsätzlich zweierlei Unterscheidungskriterien. Das erste Kriterium ist die Bindungsintensität (vgl. Doluschitz 2001, S. 378), das zweite hingegen erlaubt die Unterteilung der Kooperationsformen nach der Art der gemeinsamen Aktivitäten (vgl. KÖHNE 2002, S.13). Die vorgeschlagenen Unterscheidungssysteme sind eng miteinander verflochten, ihre Zusammenstellung bietet eine umfassende Darstellung der gesamten in der deutschen Landwirtschaft beachteten Kooperationsformen. Die Übersicht 3-1 ermöglicht die Klassifikation der praktikablen Kooperationsformen nach dem letztgenannten Kriterium.

Übersicht 3-1: Kooperationsformen nach der Art der gemeinsamen Aktivitäten

\begin{tabular}{|c|c|c|c|c|c|}
\hline $\begin{array}{l}\text { Art der } \\
\text { gemein. } \\
\text { Aktivität }\end{array}$ & Kooperation in Märkten & Arbeitsteilung & $\begin{array}{l}\text { Gemeinsame } \\
\text { Nutzung von } \\
\text { Arbeitskräften } \\
\text { und Maschinen }\end{array}$ & $\begin{array}{c}\text { Gemeinsame } \\
\text { Bewirtschaftung } \\
\text { von Betriebsteilen }\end{array}$ & $\begin{array}{c}\text { Gemeinsame } \\
\text { Bewirtschaftung } \\
\text { ganzer Betriebe }\end{array}$ \\
\hline Beispiele & $\begin{array}{c}\text { Einkaufsgemeinschaften } \\
\text { Verträge über Austausch } \\
\text { von Zwischenprodukten } \\
\text { (z.B. Wirtschaftsfutter, } \\
\text { Ferkel) } \\
\text { Verkaufs- } \\
\text { Erzeugergemeinschaften }\end{array}$ & $\begin{array}{c}\text { vertraglich } \\
\text { geregelte } \\
\text { Nachbarschaftshilfe } \\
\text { Maschinenring } \\
\text { Zusammenarbeit mit } \\
\text { Lohnunternehmen } \\
\text { Bewirtschaftungsver- } \\
\text { trag }\end{array}$ & $\begin{array}{c}\text { gemeinsame AK } \\
\text { Bruchteilsgemeinschaft } \\
\text { Maschinengesellschaft } \\
\text { Dienstleistungsgesell- } \\
\text {-schaft }\end{array}$ & $\begin{array}{c}\text { gemeinsame } \\
\text { Flächennutzung } \\
\text { gemeinsame } \\
\text { Anlagennutzung } \\
\text { (z.B. Düngerlager, } \\
\text { Getreidetrocknung } \\
\text { und Lagerung) } \\
\text { Gemeinschaftsställe }\end{array}$ & $\begin{array}{l}\text { Betriebsgesellschaft } \\
\text { aus ehemals } \\
\text { selbständigen } \\
\text { Betrieben } \\
\text { (überwiegend in } \\
\text { Westdeutschland) } \\
\text { Neugründung von } \\
\text { Betriebsgesellschaften } \\
\text { (meistens in } \\
\text { Ostdeutschland) }\end{array}$ \\
\hline
\end{tabular}

Quelle: Köhne 2002, S.13

Den geringsten Bindungsgrad weisen Kooperationen in Märkten auf, wie z.B. Zusammenschlüsse im Beschaffungs- und Absatzbereich. Die Bindung betrifft in diesen Fällen ausschließlich den Erwerbs- und Vermarktungsbereich. Dabei werden andere 
Betriebsteile zumeist völlig eigenständig weitergeführt. Es wird hierzu generell zwischen drei Arten der Zusammenarbeit unterschieden. Bei der ersten Art handelt es sich um den gemeinsamen Einkauf von Produktionsmitteln bei bestimmten Verkaufsunternehmen. Es wird hier versucht, durch die Interessenbindung einzelner Landwirte ihre Marktposition zu verbessern. Dabei ist es von wesentlicher Bedeutung, dass die Realisation der festgelegten Ziele im Ermessen der einzelnen Beteiligten liegt. Die Entscheidungsfreiheit über die Modalitäten der einzelbetrieblichen oder gemeinsamen Anschaffung der Produktionsmittel und der Vermarktung der hergestellten Erzeugnisse bleibt i. d. R. jedem Betrieb überlassen (vgl. Theuvsen 2003, S. 3 f.). Bei der zweiten Kooperationsart handelt es sich um einen vertraglich geregelten Austausch von Zwischenprodukten und die dadurch erzielbare gegenseitige Absicherung der Beschaffung sowie des Absatzes bestimmter Produkte der beteiligten Betriebe. Die dritte Gruppe umfasst den gemeinsamen Absatz von hergestellten Erzeugnissen. Bei diesen drei Kooperationsformen wird ein besonderer Wert auf die Stärkung der Marktposition sowie Einsparung von Kosten, Zeit und Arbeit gelegt.

Eine vergleichbar geringe Bindungsintensität weisen Kooperationen im Bereich der Arbeitsteilung auf. Die erste Form der Zusammenarbeit dieses Bereiches stellt die Nachbarschaftshilfe dar. Eine Abart dieser Form ist die sog. vertraglich geregelte Nachbarschaftshilfe. Die vertragliche Regelung dieser Zusammenarbeit soll dem Zweck dienen, die Betriebsplanung $\mathrm{zu}$ erleichtern und eine formelle Basis für die monetäre Leistungsabrechnung zu schaffen. In der Nachbarschaftshilfe sowohl in ihrer ursprünglichen Form als auch auf einer vertraglich geregelten Basis können verschiedene Leistungen von einer sporadisch erfolgenden Hilfe bei relativ einfachen Arbeiten bis hin zum Einsatz von spezieller Technik erbracht werden.

Als nächste Form wird unter dieser Gruppe die Zusammenarbeit mit Lohnunternehmen (LU) subsumiert. Es handelt sich hier um die Auslagerung bestimmter Maschinenarbeiten an professionelle Unternehmen der Landtechnik, die die Arbeitsaufträge mit eigenen Maschinen und eigenem Personal erledigen. Die Zusammenarbeit dieser Form kann unterschiedliche Arbeiten umfassen, von relativ einfachen Bodenbearbeitungsmaßnahmen bis hin zur Organisation bestimmter Arbeitsketten z.B. Zuckerrübenernte und -transport oder gar einer Bewirtschaftung des gesamten Ackerlandes. Die letzte Form dieser Gruppe ist die Zusammenarbeit im Rahmen der sog. Bewirtschaftungsverträge. In diesem Fall übernimmt meistens ein aktiver Landwirt oder ein LU die Bewirtschaftung bestimmter Betriebszweige oder des ganzen Betriebes eines anderen Landwirts. Die Arbeitserledigung erfolgt hier 
entweder mit dem Inventar des Auftraggebers oder, was häufiger der Fall ist, mit dem Inventar des Bewirtschafters. Unter Umständen kann diese Bewirtschaftung eine Alternative zur Verpachtung oder Einbringung des Betriebes in eine Gesellschaft bieten.

Eine höhere Stufe des Bindungsgrades betrifft die gemeinsame Nutzung von Produktionsfaktoren, insbesondere Maschinen. Bei diesen Formen der Zusammenarbeit kommt es zur Interessenbindung einiger Landwirte mit dem Ziel, die Probleme der Kapitalaufbringung für Investitionen und im Anschluss der Auslastung von Maschinen zu bewältigen. In der Praxis wird die Zusammenarbeit auf diesem Niveau in Form von Maschinengemeinschaften (Bruchteilsgemeinschaft), Maschinengesellschaften (GbR oder $\mathrm{GmbH}$ ) oder als gesonderte Form von Dienstleistungsgesellschaften organisiert. Im Fall einer Bruchteilsgemeinschaft handelt es sich i.d.R. um Zusammenschlüsse von zwei oder drei Landwirten, die eine bestimmte Maschine z.B. einen Mähdrescher oder eine Pflanzenschutzspritze gemeinsam kaufen und sie dann vorwiegend in den eigenen Betrieben aber auch bei Dritten einsetzen. Die Gesellschaften liegen i.d.R. bei Zusammenarbeit mehrerer Landwirte beim Einsatz schlagkräftiger Aggregate vor. Eine Dienstleistungsgesellschaft erstreckt sich nicht nur auf den Maschineneinsatz, sondern umfasst auch den Einkauf, die Vermarktung und u.U. weitere Tätigkeiten. Dabei beschränkt sie sich nicht notwendigerweise auf ihre Mitglieder.

In der nächsten Intensitätsstufe kann die überbetriebliche Zusammenarbeit die gemeinsame Bewirtschaftung von Betriebsteilen umfassen. Hierzu zählen ferner die gemeinsame Nutzung der Flächen und Einrichtungen wie z.B. Getreidetrocknungsanlagen, Düngerlager, Wachsstellen oder Brückenwagen und schließlich die Führung von Gemeinschaftsställen.

Die höchste Stufe der Bindung im Rahmen der landwirtschaftlichen Zusammenarbeit betrifft die Vollfusionen. In diesem Fall handelt es sich um einen Vorgang, in dem die bisher selbständig agierenden Landwirte ihre Selbständigkeit aufgeben und zusammen mit anderen Betrieben ein neues, i.d.R. größeres Unternehmen gründen. Diese Form der Zusammenarbeit bietet ein sehr breites Spektrum an Vorteilen. Ein wichtiges Ziel der Vollfusionen ist es, die Voraussetzungen für die Verbesserung der Auslastung und dadurch die Steigerung der Entlohnung der vorhandenen Produktionsfaktoren durch die Aktivierung der Größeneffekte zu schaffen. Darüber hinaus können in Vollfusionen die Möglichkeiten erschlossen werden, jene Entwicklungsschritte vollzubringen, die einzelbetrieblich oder in einer anderen Kooperationsform, sei es aufgrund niedriger Kapitalverfügbarkeit oder Einschränkungen 
durch wirtschaftliche Rahmenbedingungen nicht realisierbar wären. Von Bedeutung bei dieser Form der Zusammenarbeit sind auch die sozialen Vorteile, die z.B. in Verbesserung der Arbeitsbedingungen, der Funktionsteilung und Schaffung von Freiräumen für die Entwicklung in bestimmten inner- oder außerbetrieblichen Bereichen zum Ausdruck kommen. Die Vollfusionen kommen auch zwischen aktiven und nicht aktiven Landwirten zustande. Auf der Seite der aktiven Kooperationspartner bestehen die Vorteile in erster Linie in der Erweiterung der Produktionsfaktoren, ohne für die Kosten der Pacht und/oder der Anschaffung von Wirtschaftsgütern (z.B. Lieferrechte, Gebäude) aufkommen zu müssen. Bei den nicht aktiven Landwirten erleichtert diese Form der Zusammenarbeit oft die Betriebsaufgabe, ohne dabei die vorhandenen Produktionsfaktoren zu verkaufen oder zu verpachten. Dies ist besonders wichtig für Landwirte, die an dem Erhalt der Vermögenssubstanz und Existenzsicherung des Betriebes interessiert sind. Die Vorteilhaftigkeit der Zusammenarbeit für die nicht aktiven Landwirte wird oft von einer anderweitigen Verwertung von freigesetzten Zeitkapazitäten mitbestimmt. Bei allen Vorteilen der Vollfusionen, die hier nur angeschnitten wurden, ist zu bemerken, dass diese Form der Zusammenarbeit auch ein besonders hohes Konfliktpotenzial birgt.

\subsection{Rechtsrahmen für Kooperationen in Deutschland}

Die rechtlichen Möglichkeiten für die Ausgestaltung einer Kooperation sind in dem deutschen Rechtssystem sehr umfangreich. Die Typenspanne von Rechtsformen, in denen Kooperationen rechtlich betrieben werden, beinhaltet Personengesellschaften, juristische Personen sowie Mischformen. Eine Übersicht der Rechtsformen des deutschen Gesellschaftsrechts stellt die Abbildung 3-1 dar. 
Abbildung 3-1: Rechtsformen für kooperative Unternehmen

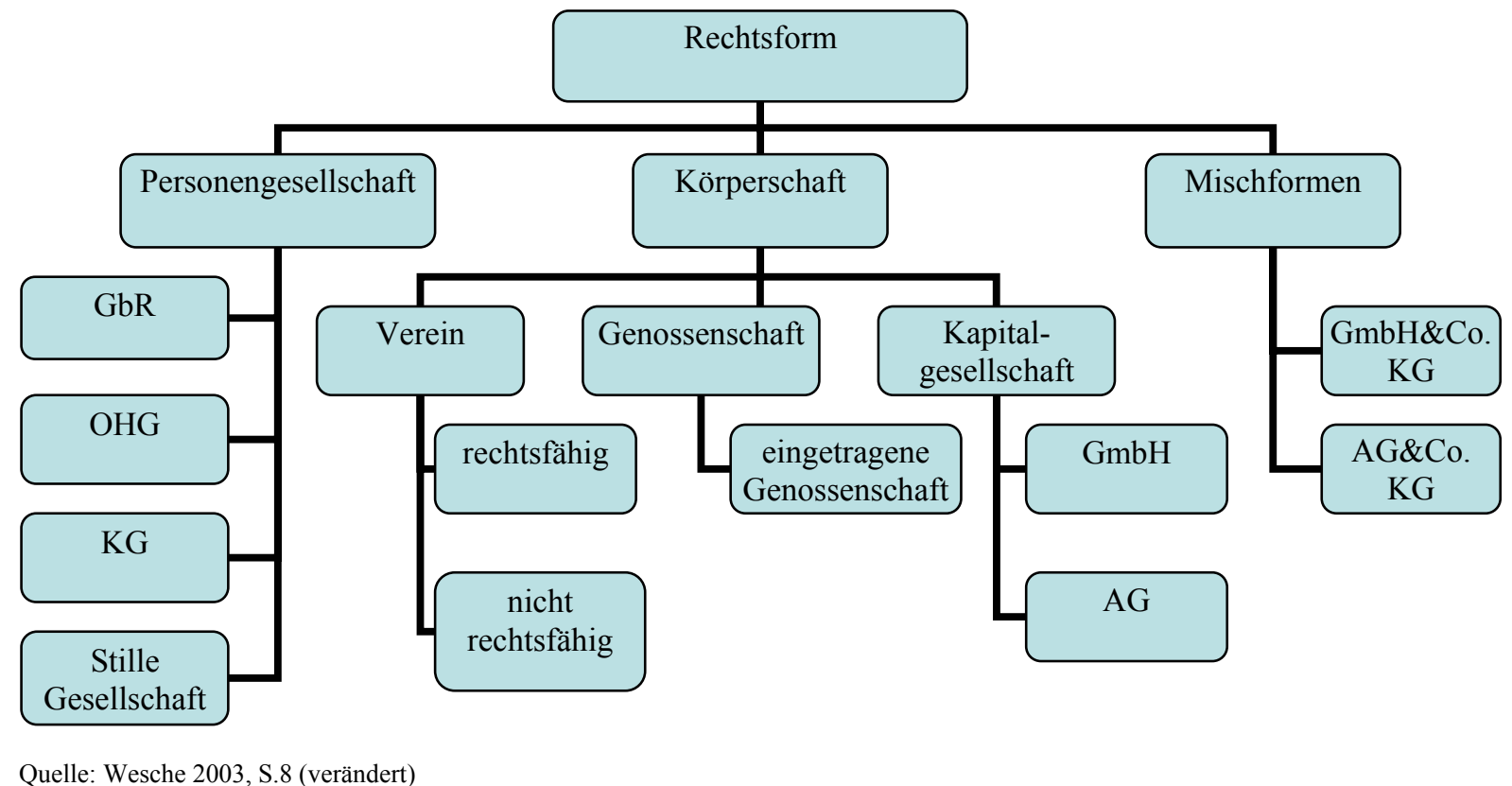

Die dargestellten Rechtsformen unterscheiden sich stark voneinander. Ihre Eignung für das Verfolgen eines bestimmten wirtschaftlichen Zwecks hängt von den Gegebenheiten des Unternehmens ab. Bei der Wahl einer konkreten Rechtsform werden unterschiedliche Kriterien mit berücksichtigt wie z.B. Beteiligungsform, Haftung, steuerliche Veranlagung, Möglichkeit der Inanspruchnahme von Fördermitteln (siehe Anhang A. 3).

Bei Kooperationen im Bereich der Maschinenutzung spielen v.a. Bruchteilsgemeinschaften, GbR sowie Vereine eine wesentliche Rolle. Eine Bedeutung kann hier auch einer GmbH zukommen. In der Praxis kommt diese Rechtsform u.a. beim Bau von gemeinsamen Biogasanlagen oder bei Tochtergesellschaften der Maschinenringe vor. Als Beispiel hierzu kann auch die überbetriebliche Organisation der Arbeitsketten von Zuckerrübenernte und transport angeführt werden. Da diese Rechtsform auf der Ebene der einzelnen landwirtschaftlichen Betriebe in Westdeutschland generell eine unerhebliche Bedeutung besitzt, wird auf sie im weiteren Teil nicht näher eingegangen. Im Folgenden wird lediglich der Rechtsrahmen von Bruchteilsgemeinschaft, GbR und eingetragenem Verein in groben Zügen skizziert ${ }^{12}$.

12 Für eine umfangreichere Studie der Rechtsformen landwirtschaftlicher Unternehmen wird auf die Veröffentlichungen von Grimm 2004, Wesche 2003 oder Winkler 1997 hingewiesen. 


\section{Bruchteilsgemeinschaft}

An der ersten Stelle der Rechtsformen, die im Bereich der kooperativen Maschinennutzung zur Anwendung kommen, wird die i.S.d. $\S \S 741 \mathrm{ff}$. BGB beschriebene Bruchteilsgemeinschaft skizziert. Die Bruchteilsgemeinschaft ähnelt von der Struktur weitgehend der GbR. Die beiden Formen unterscheiden sich im Wesentlichen im Hinblick auf die Beteiligung, Haftung und steuerliche Abgrenzung. Die Gründung einer Bruchteilsgemeinschaft bedarf keiner schriftlichen Form, somit kann sie mündlich oder sogar durch schlüssige Handlung erfolgen. Die Ein- und Verkaufstätigkeiten können die Bruchteilseigentümer ausüben. Zu den wesentlichen Eigenschaften dieser Form zählen:

- die in eine Bruchteilsgemeinschaft eingebrachten Anteile bilden im Gegensatz zur GbR kein Gesamthandseigentum, sondern sog. Bruchteilsvermögen. Im Fall einer Maschine sind das fiktive Bruchteile, die den einzelnen Gesellschaftern gehören,

- die Bruchteilsgemeinschaft ist aus der Einkommensteuersicht als ein nichteigenständiges Steuerobjekt zu betrachten. Die Bruchteile werden bei jedem einzelnen Mitglied ausgewiesen,

- $\quad$ die Rechte der einzelnen Mitglieder stehen hier im Vordergrund, es besteht dabei allerdings die Möglichkeit der Anteilsveräußerung (vgl. KÜBLER 1994, S. 35),

- die Gründung einer Bruchteilsgemeinschaft ist im Gegensatz zur Gründung einer GmbH nicht per se dem Übergang von Landwirtschaft zu Gewerbe gleich,

- das Recht auf die landwirtschaftliche Förderung einzelner Betriebe ist in der Bruchteilsgemeinschaft nicht beeinträchtigt,

- die Haftung der Bruchteilseigentümer für Verbindlichkeiten ist unbeschränkt, wenn nicht anders vereinbart worden ist.

Da die Bruchteilsgemeinschaft relativ einfach zu handhaben ist, hat sie besondere Bedeutung bei Kleingemeinschaften mit einer geringen Mitgliederanzahl.

\section{Gesellschaft bürgerlichen Rechts}

Die im bürgerlichen Gesetzbuch (BGB) unter $§ \S 705$ ff. verankerte Gesellschaft bürgerlichen Rechts wird allgemein als eine Personenvereinigung zum Erreichen eines gemeinsamen Zwecks bezeichnet. Die Eigenschaften dieser Rechtsform lassen sich komprimiert, wie folgt, auflisten: 
- $\quad$ die Gründung der Gesellschaft sowie die Herstellung ihres Vertrages bedürfen wie bei der Bruchteilsgemeinschaft keiner besonderen Form. Der Gesellschaftsvertrag kann mündlich oder sogar konkludent abgeschlossen werden (vgl. GRIMM 2004, S. 281),

- alle Gesellschafter der GbR verfügen über gleiche Rechte. Sie sind in puncto Geschäftsführung und Haftung in gleichem Maße berechtigt und verpflichtet,

- $\quad$ da die GbR wie die Bruchteilsgemeinschaft keine juristische Person ist, besitzt sie auch keine besonderen Leitungsorgane (vgl. KüBLER 1994, S. 68),

- $\quad$ der Beitrag, der bei der Entstehung einer GbR einzubringen ist, kann die Zahlung von Geld, Überlassung von Gegenständen oder Einbringung von Dienstleistungen, Herstellung eines Werkes oder Verschaffung von sonstigen Rechten und Vermögenswerten umfassen. Die Gesellschafter können verschiedene Anteile in Form von z.B. Kapital, Maschinen oder Arbeit in die GbR einbringen. Es bleibt den Gesellschaftern überlassen über die Höhe der $\mathrm{zu}$ leistenden Beiträge zu entscheiden sowie die Verteilungsregeln von Gewinn oder Verlust festzulegen,

- $\quad$ die in die Gesellschaft eingebrachten Anteile bilden Gesamthandseigentum. Dies schließt die alleinige Verfügung über das Vermögen ohne Zustimmung der anderen Gesellschafter aus,

- $\quad$ in der GbR werden die Anschaffungs- und weiteren Unterhaltungskosten nicht wie bei Bruchteilsgemeinschaft bei jedem einzelnen Mitglied, sondern in einer getrennten Buchführung verzeichnet.

Als Nachteil einer GbR kann die unbeschränkte Haftung betrachtet werden, nach der alle Gesellschafter als Gesamtschuldner sowohl mit dem Gesellschafts- als auch mit dem Privatvermögen für auftretende Schulden einstehen. Da bei der Maschinengesellschaft wegen des Arbeitsumfanges aus steuerlicher Sicht häufig der Rahmen der landwirtschaftlichen Tätigkeit überschritten wird, sind weiterhin die Abgrenzungskriterien zwischen Landwirtschaft und Gewerbe besonders zu beachten (vgl. z.B. DoLUSCHITZ, SchwenNingER 2003, S. 157; WESCHE 2003, S. 29 ff.).

\section{Verein}

Ein Verein ist eine auf die Erreichung eines gemeinsamen, privatautonom gesetzten Zwecks gerichtete, körperschaftlich verfasste Personenvereinigung. Es wird grundsätzlich zwischen rechtsfähigen und nichtrechtsfähigen Vereinen unterschieden. Relevant für den überbetrieblichen Maschineneinsatz ist der rechtsfähige Verein. Die rechtsfähigen Vereine 
werden in eingetragene Vereine (e.V.) und wirtschaftliche Vereine (w.V.) unterteilt. Dabei kommt für spezifische Zwecke der Land- und Forstwirtschaft der w.V. zum Tragen. Eine Ausnahme bilden hier die als e.V. agierenden Maschinenringe. Die Organisationen dieser Form verfolgen zwar wirtschaftliche Zwecke, begehen aber in diesem Punkt keinen Rechtsverstoß, weil sie keinen Geschäftsbetrieb führen, in Ausübung ihrer Tätigkeit prinzipiell über den Bereich der Mitgliedsbetriebe nicht hinausgehen und keine Gewinne erzielen. Die vereinfachte Charakteristik der Vereine wurde in der Übersicht 3-2 dargestellt.

Übersicht 3-2: Vereinsformen

\begin{tabular}{|c|c|c|c|c|}
\hline & Zweck & Haftung & Erforderliches Vermögen & $\begin{array}{c}\text { Erforderliche } \\
\text { Mitgliederzahl }\end{array}$ \\
\hline $\begin{array}{c}\text { eingetragener } \\
\text { Verein (e.V.) }\end{array}$ & $\begin{array}{c}\text { nicht } \\
\text { wirtschaftlich }\end{array}$ & Vereinsvermögen & kein & mindestens 7 \\
wirtschaftlicher \\
$\begin{array}{c}\text { Verein (w.V.) } \\
\text { wirtschaftlich }\end{array}$ & Vereinsvermögen & $\begin{array}{c}\text { Voraussetzung für Verleihung der } \\
\text { Rechtsform juristischer Person } \\
\text { (verschiedener Betrag, generell an } \\
\text { GmbH angenähert ca. 25 000EUR) }\end{array}$ & mindestens 7 \\
\hline
\end{tabular}

Zu den wichtigsten Eigenschaften eines Vereins zählen:

- $\quad$ ein Verein tritt unter dem eigenen Namen auf und ist vom Wechsel der Mitglieder weniger abhängig als andere Rechtsformen,

- $\quad$ ein eingetragener Verein erwirbt die Rechte einer juristischen Person allein durch die Anmeldung zum Vereinsregister,

- $\quad$ im Gegensatz zur GbR bilden die ins Vereinsvermögen eingebrachten Anteile kein Gesamthandseigentum, was die Verfügungsfreiheit unberührt lässt,

- die vorgeschriebenen Organe sind Mitgliederversammlung und Vorstand. Bei Maschinenringen liegen in der Praxis zusätzlich ein Ausschuss und ein Beirat vor.

$\mathrm{Zu}$ den wichtigsten Rechten und Pflichten der Organe eines Vereins zählen (vgl. KÜBLER 1994, S. 241):

a. Mitgliederversammlung:

- Bestellung und Entlastung des Vorstandes,

- Änderung des Vereinszwecks (erforderlich ist die Zustimmung aller Mitglieder) oder der Satzung (bei wenigstens Dreiviertelmehrheit der erschienen Mitglieder), 
- die Entscheidung in allen den Verein berührenden Fragen bis hin zur tatsächlichen Vereinsführung z.B.:

- $\quad$ Festsetzung der Mitgliederbeiträge und Verrechnungssätze,

- $\quad$ Entscheidung über Aufnahme und Ausschluss von Mitgliedern,

- $\quad$ Genehmigung des Jahresabschlusses.

b. Vorstand:

- Vertretung des Vereins nach außen,

- Erledigung der normalen Angelegenheiten z.B.:

- Vermittlung der Maschinen,

- $\quad$ Berechnung der Dienstleistungskosten.

c. Ausschuss:

- Entscheidungen in allen Angelegenheiten, die nicht den anderen Organen unterliegen.

d. Beirat:

- Beratung des Ausschusses.

Die Mitgliedschaft in einem e.V. endet im Fall von Austritt, Ausschluss oder Tod. Die Mitgliederrechte sind nicht übertragbar und nicht vererblich. Die Beendigung des Vereins erfolgt prinzipiell durch Verlust der Rechtsfähigkeit und Auflösung des Vereins. Anders als bei der GbR bedeutet das Ausscheiden von einzelnen Mitgliedern nicht die Auflösung des Vereins (vgl. KÜBLER 1994, S.171).

\subsection{Entstehung, aktuelle Bedeutung und weitere Entwicklung verschiedener Formen der überbetrieblichen Zusammenarbeit beim Maschineneinsatz}

Die Mechanisierung spielt in der Landwirtschaftsentwicklung schon seit Jahrzehnten eine gewichtige Rolle. Der Einsatz von moderner Technik war seit dem Übergang von pferdegezogenen Landmaschinen am Anfang des zwanzigsten Jahrhunderts bis zur derzeitigen Verbreitung moderner Verfahren mit erheblichen ökonomischorganisatorischen Problemen verbunden. In Deutschland übte der, in den fünfziger Jahren angefangene wirtschaftliche Aufschwung einen erheblichen Einfluss auf die gesamte Struktur der Landwirtschaft aus. Infolge der Abwanderung der $\mathrm{AK} \mathrm{zu}$ außerlandwirtschaftlichen Bereichen, der sukzessiven Vergrößerung der Betriebe, ihrer weiteren Spezialisierung sowie 
der permanent steigenden Produktionsanforderungen erlangte der Einsatz moderner leistungsfähiger Technik im Laufe der Zeit eine immer größere Bedeutung.

Die Entwicklung der Mechanisierung in Deutschland ist neben den vorher geschilderten Änderungen im Agrarsektor und den Maßnahmen der Agrarpolitik auch in einem Zusammenhang mit der Entwicklung zu analysieren. Bei Berücksichtigung dieser Faktoren zeigt sich ein Entwicklungsverlauf der in vielen Fällen in der Schaffung von erheblichen Überkapazitäten und einer überproportionalen Steigerung der Arbeitserledigungskosten mündete. Oft wurden die Maschineninvestitionen nicht vor dem Hintergrund des wirklichen Bedarfs, sondern aufgrund überzogener Selbständigkeit und teils auch Prestigedenken unter Hintanstellung der Wirtschaftlichkeit vorgenommen. Trotzdem gewann die Idee der überbetrieblichen Maschinenverwendung (ÜMV) an Bedeutung.

Das Spektrum der hier vorhandenen Formen umfasst:

- Nachbarschaftshilfe,

- $\quad$ gemeinsame Anschaffung und den Einsatz bestimmter Maschinen im Rahmen einer Gemeinschaft oder Gesellschaft,

- $\quad$ Maschinenringe (MR),

- $\quad$ Zusammenarbeit mit Lohnunternehmen (LU)

- $\quad$ Kombination der genannten Formen, z.B. die Mitarbeit von Lohnunternehmen in Maschinenringen.

Im Folgenden wird auf die Entstehung, die aktuelle Bedeutung, die spezifischen Vorteile und die weitere potenzielle Entwicklung einzelner Formen eingegangen. Für die Realisation dieser Aufgabe wurde außer der Literaturrecherche eine Expertenbefragung durchgeführt. Das Expertenforum umfasste insgesamt 26 Vertreter aus den fünf folgenden Berufsbereichen: die Lohnunternehmen, die Maschinenringe, die landwirtschaftliche Praxis, die landwirtschaftliche Beratung und die Ausbildung. Die Befragung erfolgte mündlich anhand eines Gesprächsleitfadens mit 22 Fragen, die in 4 Gruppen eingeteilt wurden (siehe Anhang A. 4.1). Es war das Ziel der Befragung, praxisrelevante Erkenntnisse zu gewinnen, die die Literaturstudie vervollständigen sollen. Wegen der relativ kleinen Stichprobe wird auf eine detaillierte explizite Analyse der Befragungsergebnisse verzichtet. Die in der Befragung gewonnenen Erkenntnisse werden in Form von Tendenzaussagen in Bezug auf die einzelnen Kooperationsformen dargestellt. 


\subsubsection{Vertraglich geregelte Nachbarschaftshilfe}

Eine ursprüngliche Form der Zusammenarbeit im landwirtschaftlichen Bereich bildet die Nachbarschaftshilfe. Unter dem Begriff der Nachbarschaftshilfe wird ein informell, ohne besondere Vereinbarung, auf Basis von Vertrauen und Gegenseitigkeit, gelegentlich i.d.R. ohne Geldfluss erfolgender Leistungsaustausch zwischen benachbarten Betrieben verstanden. Der Nachbarschaftshilfe in der herkömmlichen Form kam eine besondere Bedeutung in den fünfziger und den sechziger Jahren zu. Dies gründet sich zum einen in den damaligen Strukturen der Landwirtschaft und zum anderen in der fortschreitenden Mechanisierung, die immer häufiger den Einzug in den kleinen, im Wesentlichen nur auf das Arbeitspotenzial der Familienmitglieder gerichteten Betrieben hielt (SCHMIDT 2004). Bei den arbeitsaufwendigen Verfahren bot die Nachbarschaftshilfe häufig die einzige Möglichkeit, durch den Einsatz fremder Maschinen die Arbeit effizienter zu gestalten und am technischen Forschritt teilzunehmen. Der auf der Grundlage der Nachbarschaftshilfe erfolgende Leistungsaustausch bildete in vielen Fällen eine Basis zur dauerhaften Interessenbindung der beteiligten Landwirte. Die vor diesem Hintergrund entwickelte Zusammenarbeit erreichte in einigen Fällen das Ausmaß einer formell vertraglich geregelten Nachbarschaftshilfe. Eine wichtige Eigenschaft dieser Form der Zusammenarbeit besteht darin, dass der Bindungsgrad der einzelnen Betriebe nach wie vor unveränderlich gering bleibt. Die einzelnen Maschinen, deren Leistung den Vertragsgegenstand bildet, bleiben im Eigentum der einzelnen Landwirte. Daher bestehen in diesem Fall keine Kapitalverflechtungen oder langfristigen Verpflichtungen. Der wesentliche Unterschied zwischen der herkömmlichen Nachbarschaftshilfe und der vertraglich festgelegten Zusammenarbeit besteht in der Verrechnungsmethode von Kosten und Leistungen zwischen Auftraggeber und -nehmer. Zur Vermeidung potenzieller Missverständnisse werden im Kooperationsvertrag bestimmte Kostenvoranschläge vorgenommen, die eine Grundlage für eine monetäre Leistungsabrechung bilden. Den formellen Rahmen der Zusammenarbeit gibt i.d.R. ein wechselseitiger Werksvertrag. Ein wesentlicher Unterschied zwischen diesem Vertrag und anderen Vertragsformen, wie z.B. Arbeits- oder Dienstvertrag, besteht in der Abrechnungsform. Während bei den anderen Vertragsformen die zu leistende Arbeit die Abrechungsbasis bildet, handelt es sich im Fall eines Werksvertrages um das endgültige Leistungsergebnis. Ein Beispiel eines solchen Vertrages wird in einem DLG-Beitrag dargestellt (vgl. BunDEL et al. 1989, S. 52 ff.). Aus diesem auf Ackerbaubetriebe bezogenen Entwurf lassen sich folgende relevante Punkte einer Vereinbarung ableiten: 
1. Festlegung des Auftrages und Definition der zu erbringenden Leistungen sowie des angestrebten Ergebnisses,

2. Abrechnungs- und Vergütungsmethode für erbrachte Leistungen,

3. Fristensetzungen für:

a. Vertragsdauer,

b. Bewirtschaftungsplan,

c. Kostenabrechnung,

4. Haftung und Gewährleistung,

5. Schiedsverfahren bei Meinungsverschiedenheiten über den Leistungsumfang, den Verrechnungssatz oder Schadenersatzanspruch, zu deren Lösung Dritte herangezogen werden müssen.

Der Umfang des aus dem jeweiligen Arbeitsbedarf resultierenden Maschineneinsatzes wird rechtzeitig nach gemeinsamer Absprache in Form eines Bewirtschaftungsplanes festgelegt. Die Vereinbarungen können je nach der Arbeitsart entweder einmal saisonal oder vermehrt in kurzen, z.B. wöchentlichen Abständen stattfinden. Die Abrechnung der Leistung erfolgt auf Basis von Aufzeichnungen in Tagebüchern, deren Führung und Kontrolle vor Ort festzulegen sind. Die Abrechnungsbasis bilden hier bestimmte Verrechnungssätze für geleistete Arbeit in Stunden oder ha LF. Von den primär ermittelten Preisen für Leistungen werden u.U. bestimmte Ab- und Zuschläge vorgenommen. Dies hat zum Ziel, die unterschiedlichen Kosten und den unterschiedlichen Zeitaufwand, der z.B. durch steinhaltige Fläche oder stärkere Bodendichte verursacht wird, in der Abrechnung $\mathrm{zu}$ berücksichtigen. Die Kostenabrechung kann mittelfristig z.B. für eine bestimmte Saison oder langfristig für ein Jahr erfolgen. So erfolgt die Zahlung je nach der vorliegenden Vereinbarung direkt nach der Arbeitsleistung oder in einem weiteren Zeitabstand.

Eine wesentliche Bedeutung kommt in einem Werkvertrag der Festlegung der Haftung und Gewährleistung zu. $\mathrm{Zu}$ den Posten, die an dieser Stelle zu erfassen sind, gehören die Rechtzeitigkeit sowie die Ordnungsmäßigkeit der erbrachten Leistungen. Besondere Schwierigkeiten können in der Abgrenzung der Verantwortung und der Pflicht evtl. Nachbesserungen der Leistungen bestehen.

Die Entwicklung der vertraglich geregelten Nachbarschaftshilfe kann von Betrieb zu Betrieb unterschiedlich erfolgen. Günstige Voraussetzungen für die Zusammenarbeit liegen insbesondere bei solchen Betrieben vor, die eine unterschiedliche Faktorausstattung 
aufweisen, gleichzeitig aber wegen ähnlicher Produktionsstrukturen die zwischenbetriebliche Auslastung der freien Kapazitäten ermöglichen. Bei Betrieben mit homogener Struktur ist das erforderliche Potenzial der Nachfrage und des Angebots i.d.R. nicht vorhanden. Ein erhebliches Hindernis in der Entwicklung der Nachbarschaftshilfe stellt die oben angesprochene Termingerechtheit der zu erbringenden Leistungen dar. Dies kommt besonders zum Ausdruck in Betrieben, in denen wegen weitgehend identischer Produktionsstruktur die saisonalen Arbeitsspitzen zur gleichen Zeit entstehen.

Der Nachbarschaftshilfe kam eine erhebliche Bedeutung in der Entstehungsphase der Organisation der Maschinenringe zu. Bei fortschreitender Spezialisierung der Betriebe bildete sie häufig ein Übergangsstadium zur Lohnmaschinendienstleistung oder zur weiteren Intensivierung der Kooperation in Maschinengemeinschaften (ISERMEYER 2004). Obwohl die Nachbarschaftshilfe in der Praxis in unterschiedlicher Ausprägung auch heute eine große Bedeutung besitzt, ist es angesichts fehlender statistischer Angaben schwer, konkrete Aussagen hinsichtlich der Anzahl der Fälle zu treffen. Die Verbreitung dieser Form der Zusammenarbeit hängt mit den von Region zu Region unterschiedlichen Rahmenbedingungen zusammen. Eine relativ große Bedeutung hat diese Form nach wie vor in den südlichen Regionen Deutschlands, wo die Landwirtschaftstruktur durch vergleichsweise kleine Familienbetriebe gekennzeichnet ist. Sie spielt hier eine wesentliche Rolle v.a. bei relativ einfachen und wenig aufwendigen Arbeiten. Typische Beispiele hierzu sind die gegenseitige Unterstützung bei der Heuernte oder die Nutzung von Maschinen für die Bestellung und den Pflanzenschutz. Im Fall großer Spezialmaschinen bietet diese Form der Zusammenarbeit i.d.R. keine ausreichende Sicherung der erforderlichen Kapazitätenauslastung. Dies hat zur Folge, dass sie bei aufwendigen und umfangreichen Verfahren zzt. eher nur sporadisch zum Tragen kommt (KADNER 2003).

\subsubsection{Maschinenringe}

Eine besondere Stellung in der Entwicklungsgeschichte der überbetrieblichen Maschinennutzung nimmt in der deutschen Landwirtschaft die Organisation der Maschinenringe ein. Der erste MR, ursprünglich als Maschinenbank bezeichnet, wurde im Jahr 1958 in Bayern gegründet und als ein Zusammenschluss einer größeren Anzahl von Bauern definiert. Kennzeichnend für die MR war, dass diese selbst keine eigenen Maschinen besaßen, sondern als eine Art Vermittlungsorganisation agierten und die Aufgabe, die potenzielle Nachfrage und das Angebot der Maschinenleistungen zwischen Mitgliedsbetrieben zu erschließen, verfolgten. Dementsprechend war das ursprüngliche Ziel 
dieser Organisation, unter Berücksichtigung der gegebenen Betriebsstrukturen, den überbetrieblichen gewinnwirksamen Austausch der freien Maschinen- oder Arbeitskapazitäten zu fördern und dadurch zur Vermeidung von teuren Investitionen beizutragen (vgl. GEIERSBERGER 2000, S. 14).

Signifikant für die Entwicklungsphase der Organisation ist die Gründung von zahlreichen kleinen, überwiegend von nebenberuflichen Geschäftsführern geleiteten MR. So nahm die Zahl der MR in Westdeutschland in den Jahren 1960-1965 von 23 auf 502 zu. Trotz der zahlenmäßig starken Verbreitung der MR, blieb die Beteiligung der Landwirte an den Organisationen sehr klein. Im Jahr 1970 waren lediglich 4 \% aller Landwirte in MR vereint. Ein Umschwung in der Entwicklung der Organisation zeichnete sich seit dem Anfang der siebziger Jahre ab. Während im Jahr 1970 auf dem gesamten Gebiet der alten Bundesländer noch 751 MR existierten, blieben im Jahr 1980 lediglich 259 MR bestehen. Die weitere Entwicklung der MR vollzog sich verstärkt durch die Vergrößerung des Flächenumfangs und die Zunahme der Mitgliederanzahl. So wurde der Anteil der an den MR beteiligten landwirtschaftlichen Betriebe bis 1990 nahezu verdreifacht. Folgerichtig stieg der Flächenumfang von 1 Mio. ha LF im Jahr 1970 auf 7,1 Mio. ha LF im Jahr 2003. An den Anteilen der gesamten LF gemessen, bedeutet das eine Zunahme von $8 \%$ auf insgesamt $62 \%$ (siehe Tabelle 3-1).

Tabelle 3-1: Entwicklung der MR im früheren Bundesgebiet in den Jahren 1960-2003

\begin{tabular}{|c|c|c|c|c|c|c|}
\hline Jahr & $\begin{array}{c}\text { Anzahl } \\
\text { der MR }\end{array}$ & $\begin{array}{c}\text { davon mit } \\
\text { hauptberuflichem } \\
\text { Geschäftsführer }\end{array}$ & $\begin{array}{c}\text { Anzahl der } \\
\text { Mitglieder }\end{array}$ & $\begin{array}{c}\text { Anteil an } \\
\text { allen Betrieben }\end{array}$ & $\begin{array}{c}\text { LF in MR } \\
\text { in } 1000 \text { ha }\end{array}$ & $\begin{array}{c}\text { Anteil an } \\
\text { gesamter LF } \\
\text { in } \%\end{array}$ \\
\hline 1960 & 23 & 1 & - & - & - & - \\
1965 & 502 & 13 & - & - & - & 8 \\
1970 & 751 & 72 & 45000 & 4 & 1000 & 19 \\
1975 & 271 & 164 & 114260 & 14,3 & 3174 & 26 \\
1980 & 259 & 161 & 181206 & 18,5 & 5047 & 30 \\
1990 & 284 & 184 & 195870 & 44 & 6558 & 38 \\
2000 & 228 & 216 & 194132 & 48,5 & 7147 & 62 \\
2003 & 234 & 191 & &
\end{tabular}

Die Entwicklung der MR verlief vor dem Hintergrund der differenzierten Strukturen bestimmter Regionen unterschiedlich. Besonders günstige Entwicklungsbedingungen fand diese Organisation in den südlichen Bundesländern, insbesondere in Bayern, wo die 
Landwirtschaftstruktur geschichtlich bedingt sehr stark durch kleine Familienbetriebe geprägt wurde. Eine wesentliche Bedeutung für die weitere Verbreitung der Organisation gewann die gegen Ende der sechziger Jahre in einzelnen Ländern eingeführte finanzielle Förderung. Die Förderung erfolgte ursprünglich durch eine teilweise Übernahme der Organisationskosten in Form einer sog. Starthilfe (vgl. ISERMEYER 1981, S.37). In den Jahren 1968-1972 beteiligte sich zum erheblichen Teil der Bund an der Förderung der MR. So wurden die Fördermittel zu ca. $60 \%$ durch den Bund und zu $40 \%$ durch einzelne Länder bereitgestellt. In dem angesprochenen Programm flossen den MR insgesamt 1,7 Mio. DM zu. Der Förderbetrag bewegte sich je nach Bundesland auf unterschiedlichem Niveau. Die meisten Fördermittel kamen den MR in Bayern zu. Die Förderungsquote in diesem Bundesland übertraf ungefähr 80-mal den Betrag, der in diesem Programm den MR in Hessen zugeteilt wurde (vgl. CRÖNLEIN 1979, S. 70).

Das in dem Förderungsprogramm designierte Ziel, eine weitere Vergrößerung der einzelnen MR zu beschleunigen, erzielte besondere Effekte in Bayern und in Rheinland-Pfalz. In diesen Ländern wurden teilweise schon in den siebziger Jahren MR mit über 1.000 Mitgliedern entwickelt. Signifikant für die damalige Mitgliederstruktur der MR war eine relativ hohe Präsenz größerer Betriebe bei geringer Vertretung der flächenmäßig kleineren Betriebe. Während die durchschnittliche Betriebsgröße eines landwirtschaftlichen Betriebes in Bayern im Jahr 1978 bei 12,6 ha LF lag, verfügten die Mitgliedsbetriebe der MR zu dieser Zeit über durchschnittlich 20,4 ha LF. Die Gruppe der Vollerwerbsbetriebe bildete ca. $62 \%$ aller Mitgliedsbetriebe. Die Anteile der Nebenerwerbsbetriebe an der Mitgliederanzahl erreichten nur ca. 20 \% (vgl. GRIMM 1980, S. 34).

Die damalige Entwicklung der MR schlägt sich auch in ihrer Führungsform nieder. Im Laufe der Zeit kam es zu zahlreichen Zusammenschlüssen von kleineren nebenberuflich geführten MR. Die auf diesem Wege umgewandelten MR wurden fast ausschließlich durch hauptberufliche Geschäftsführer geleitet. So verblieb beispielhaft im Jahr 1979 von den insgesamt $90 \mathrm{MR}$ in Bayern lediglich einer, der nebenberuflich geführt wurde (vgl. ISERMEYER 1981, S.38). An dieser Stelle ist $\mathrm{zu}$ bemerken, dass das bayerische Landwirtschaftförderungsgesetz einen wesentlichen Beitrag zu dieser Entwicklung leistete. Demgemäß wurde die hauptberufliche Führung zwingend erforderlich, damit ein MR als Mitglied einer Landesvereinigung registriert werden und eine staatliche Förderung beantragen konnte (vgl. BAYLwFÖG Art. 8). 
Mit der allmählichen Minderung der Teilnahme des Bundes an der Förderung der MR und der Übernahme der Finanzierungslast durch die einzelnen Länder, wurde das ursprünglich definierte Ziel, ausschließlich hauptberufliche $\mathrm{MR} \mathrm{zu}$ fördern, teilweise gelockert. In den Ländern Hessen, Nordrhein - Westfalen und Baden - Württemberg wurde bei der Förderung fortan nicht mehr zwischen den neben- und hauptberuflich geführten MR differenziert. Die deutliche Dominanz der MR mit einem hauptberuflichen Geschäftsführer wurde dadurch generell zwar nicht beeinträchtig. Diese Regelung leistete jedoch einen Beitrag zu einer gleichzeitigen Weiterentwicklung der beiden Varianten der MR. Ein besonderes Beispiel bildet die Struktur der MR in Baden - Württemberg. Zu dieser Zeit gab es in diesem Land rund $33 \mathrm{MR}$, davon wurde nur einer hauptberuflich geführt.

Die Entwicklung der MR mit haupt- oder nebenberuflicher Geschäftsführung kann in einem engen Zusammenhang mit der von Region zu Region unterschiedlichen Verbreitung anderer Kooperationsformen analysiert werden. In Regionen mit einer starken Verbreitung der ÜMV oder mit Angebot der Fremdmaschinenarbeit fanden v.a. die nebenberuflich geführten Maschinenringe günstige Entwicklungsbedingungen. Die Funktion der MR beschränkte sich in diesen Regionen über längere Zeit im Wesentlichen auf die Unterstützung des vorhandenen Leistungsaustausches. Dadurch lässt sich die Struktur der MR in Baden-Württemberg erklären. Die MR hatten in ihrer Entwicklungsphase in diesem Bundesland eine besonders starke Konkurrenz in Maschinengemeinschaften (vgl. CRÖNLEIN 1979, S. 103). Zu Regionen mit einem großen Angebot an Fremdmaschinenleistung zählten schon damals Schleswig Holstein und der nördliche Teil von Niedersachsen. Die Tätigkeit der MR verstärkte hier die Tendenz weiterer Spezialisierung und Aufteilung auf Leistungsempfänger auf der einen Seite und Betriebe, die als professionelle Dienstleister agierten auf der anderen Seite. In Regionen, wo der Maschineneinsatz vornehmlich im Rahmen der Eigenmechanisierung erfolgte und keine anderen Formen der Mechanisierung verbreitet waren, mussten zunächst Voraussetzungen geschaffen werden, die potenzielle Nachfrage und das Angebot der Fremdmaschinenleistung zu erschließen. Eine gewichtige Bedeutung kam in diesem Vorgang der hauptberuflichen Geschäftsführung der MR zu (vgl. ISERMEYER 1981, S.38).

Die Unterschiede in der Entwicklung der MR in den einzelnen Bundesländern bleiben auch gegenwärtig erkennbar. Zahlenmäßig sind die MR mit deutlichem Abstand am stärksten in den südlichen Bundesländern vertreten. Allein in Bayern, Baden - Württemberg und Hessen befinden sich zzt. über $58 \%$ aller MR Deutschlands. Die höchsten Anteile der Mitgliedsbetriebe weisen die MR in Nordrhein - Westfalen und Bayern auf. Demzufolge 
befanden sich in der Bewirtschaftung der Mitgliedsbetriebe der MR dieser Länder jeweils $76 \%$ bzw. $82 \%$ der gesamten LF.

Die heutigen Differenzen in der Entwicklungsstruktur der MR in den einzelnen Bundesländern resultieren nach wie vor aus den früher erläuteten, regional unterschiedlichen Rahmenbedingungen der Landwirtschaft. In Bayern, wo zzt. fast ein Drittel aller Maschinenringe Deutschlands aktiv ist, dominieren nach wie vor bäuerliche Familienbetriebe mit einer relativ kleinen Flächenausstattung und einem großen Anteil an Grünland. Die Maschinenarbeitserledigung erfolgt in diesen Betrieben traditionell bedingt überwiegend in Eigenmechanisierung. Unter diesen Bedingungen bietet die Zusammenarbeit mit einem MR eine Möglichkeit, den Maschinenauslastungsgrad zu steigern und dadurch die Effizienz der Mechanisierung zu verbessern.

In Norddeutschland, wo größere Betriebe vorherrschen, allen voran in Schleswig- Holstein, und im nördlichen Teil Niedersachsens, spielt die in MR erschlossene Nachbarschaftshilfe einzelner Landwirte eine relativ geringe Rolle. In den großstrukturierten Ackerbaubetrieben dieser Regionen kann die Eigenmechanisierung weitaus effizienter gestaltet werden als in den kleinen Betrieben der südlichen Regionen. Weiterhin liegen in den zahlreich in dieser Region vertretenen spezialisierten Veredlungsbetrieben günstige Voraussetzungen vor, bestimmte Arbeiten an Dritte auszulagern. Geschichtlich bedingt entwickelte sich in dieser Region im Bereich der Maschinennutzung eine spezifische Form der Zusammenarbeit zwischen den einzelnen Betrieben. Dabei handelt es sich um eine schrittweise Ausbildung der Abhängigkeitsstrukturen zwischen Betrieben des Leistungsangebotes und der -nachfrage.

Eine relativ niedrige Bedeutung haben die $M R$ in den östlichen Bundesländern. Die MR dieser Regionen sind zwar flächenmäßig größer als die MR der westlichen Bundesländer, wird jedoch der Flächenumfang der Mitgliedsbetriebe den gesamten LF der Länder gegenübergestellt, so zeigt sich, dass die MR trotz einer Aufwärtsentwicklung lediglich einen relativ kleinen Teil der LF dieser Regionen erschließen (siehe Tabelle 3-2). Aufgrund der Betriebsgrößenstrukturen in diesen Regionen spielt die Maschinenarbeitsvermittlung in den MR eine geringe Rolle. Dies betrifft auch die Organisation der aus Westdeutschland bekannten, von MR geführten, größeren Maschinengemeinschaften (siehe hierzu S. 64). An Bedeutung in den MR der östlichen Bundesländer nimmt demgegenüber ständig die Organisation des Einsatzes der Betriebshelfer in den landwirtschaftlichen Betrieben zu (Ahnert 2006; siehe hierzu auch S. 55 f.). 
Tabelle 3-2: Maschinenringe in ausgewählten Bundesländern in den Jahren 1979-2003

\begin{tabular}{|c|c|c|c|c|c|c|c|c|c|c|c|}
\hline Bundesland & 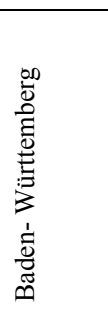 & 竞 & 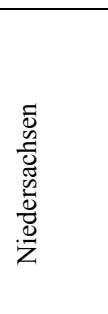 & 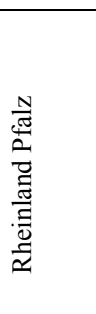 & 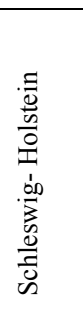 & 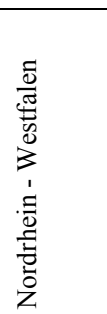 & 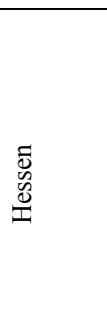 & 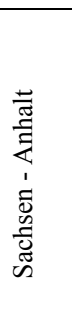 & 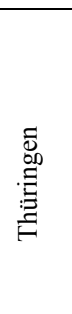 & $\begin{array}{c}\tilde{D} \\
\overline{0} \\
\overline{\tilde{c}} \\
\tilde{n} \\
\tilde{n}\end{array}$ & 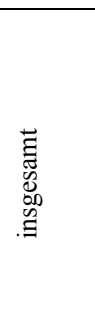 \\
\hline 1979 & & & & & & & & & & & \\
\hline Anzahl der MR & 34 & 90 & 65 & 20 & 13 & 37 & - & & - & - & 259 \\
\hline davon hauptberufliche MR & 1 & 89 & 41 & 12 & 6 & 12 & - & & - & - & 161 \\
\hline Mitglieder insgesamt & 8007 & 64357 & 15524 & 9157 & 3124 & 17560 & - & - & - & - & 117729 \\
\hline Anteil an allen Betrieben & 5,6 & 23,4 & 12 & 13 & 10,3 & 27 & - & - & - & - & 14,7 \\
\hline $\begin{array}{l}\text { Verrechnungswert } \mathrm{DM} / \mathrm{ha} \\
\text { davon in } \%\end{array}$ & 76 & 82 & 75 & 56 & 58 & 47 & - & - & - & - & 67 \\
\hline Betriebshilfe & 15 & 23 & 5 & 19 & 5 & 60 & - & - & - & - & - \\
\hline Maschinenvermittlung & 85 & 77 & 95 & 81 & 95 & 40 & - & - & - & - & - \\
\hline 2003 & & & & & & & & & & & \\
\hline Anzahl der MR & 31 & 76 & 37 & 14 & 12 & 19 & 45 & 6 & 10 & 10 & 260 \\
\hline davon hauptberufliche MR & 29 & 76 & 36 & 10 & 12 & 13 & 15 & 6 & 10 & 10 & 212 \\
\hline Mitglieder insgesamt & 25400 & 100500 & 23300 & 10800 & 6500 & 17300 & 10300 & 900 & 900 & 850 & 196750 \\
\hline Anteil aller Betriebe in $\%$ & 41,2 & 77,0 & 48 & 47,9 & 34 & 85 & 38,0 & 17,9 & 20,4 & 18,0 & 50,6 \\
\hline $\begin{array}{l}\text { Verrechnungswert EUR/ha } \\
\text { Davon in } \%\end{array}$ & 100,5 & 115 & 103 & 230 & 52 & 32,5 & 97 & 17 & 14 & 18 & 92 \\
\hline Betriebshilfe & 12 & 15 & 3 & 3 & 9 & 45 & 1 & 20 & 19 & 1 & 12 \\
\hline Maschinenvermittlung & 31 & 69 & 54 & 60 & 34 & 45 & 53 & 54 & 81 & 98 & 57 \\
\hline Tochtergesellschaften & 57 & 16 & 43 & 37 & 57 & 10 & 46 & 26 & - & 1 & 31 \\
\hline LF in den MR in 1000 ha & 997 & 2687 & 1330 & 364 & 452 & 980 & 338 & 334 & 319 & 116 & 7917 \\
\hline Anteil an gesamter LF in \% & 69 & 82 & 45 & 52 & 44 & 76 & 44 & 29 & 40 & 15 & 47 \\
\hline
\end{tabular}

Quelle: Isermeyer 1981, S.39 nach KTBL; BMR e.V. 2004

Von der bereits geschilderten regional unterschiedlichen Verbreitung der MR in bestimmten Bundesländern abgesehen, bleibt das Gefüge und das herkömmliche Ziel der Organisation, die Maschinenarbeit zu vermitteln, unverändert. Die bevorzugte Rechtsform ist hier nach wie vor der e.V.. Für die Leitung eines MR ist ein Geschäftsführer zuständig. Die Geschäftsführer besitzen heute meistens den Status hauptberuflich tätiger Mitarbeiter und werden auf Basis von Umsatzprovision bezahlt. In den früheren Jahren wurde die Finanzierung der Stelle des Geschäftsführers, zumindest teilweise in das Programm der MR- Förderung eingebunden. Derzeit wird in jedem einzelnen Land getrennt entschieden, ob MR bezuschusst werden und ggf. in welcher Form dies erfolgen wird. Generell kann festgehalten werden, dass die MR heutzutage im Vergleich zu früheren Jahren in einem eher nur geringen Maße gefördert 
werden. Eine wesentliche Bedeutung spielt die Förderung der MR nach wie vor in Bayern. Diese Förderung findet rechtliche Grundlage in dem bayrischen Landwirtschaftsförderungsgesetz. Laut Art. 8 dieses Gesetzes können die MR als Selbsthilfeeinrichtungen in den Genuss der staatlichen Fördermittel gelangen. Dies bedeutet allerdings nicht, dass die Fördermittel den einzelnen MR unmittelbar zufließen, sondern vielmehr, dass sie in erster Linie einer staatlich anerkannten Vereinigung, dem Kuratorium der bayerischen Maschinenringe (KBM), zur Verfügung gestellt werden. Von dieser Stelle aus werden die Fördermittel an die einzelnen MR verteilt. Bis zum Jahr 2001 bestand die Förderung in der Erstattung von bis zu $70 \%$ der anfallenden Personalkosten (hierzu zählte z.B. das Gehalt des Geschäftsführers) und bis zu 40 \% der Geschäftskosten. Im Jahr 2001 wurde die institutionelle Förderung als Prozentsatz der anfallenden Kosten anhand eines Förderungsvertrages mit dem bayerischen Staatsministerium für Landwirtschaft und Forsten (StMLF) in eine Programmförderung von drei Haupttätigkeitsbereichen überführt. Hierzu zählt die Organisation:

- $\quad$ der zwischenbetrieblichen nebenberuflichen sozialen Betriebshilfe,

- $\quad$ der zwischenbetrieblichen wirtschaftlichen Betriebshilfe,

- $\quad$ des zwischenbetrieblichen Maschineneinsatzes (einschließlich Bedingungspersonal).

Gemäß der ursprünglichen Vereinbarung wurde die anfängliche Erstattungsquote von $40 \%$ der anfallenden Kosten der o.g. Bereiche nach fünf Jahren auf 33 \% reduziert.

Jedes Mitglied eines MR hat einen finanziellen Beitrag zu leisten. Die Höhe dieser Beiträge ist vor Ort satzungsmäßig festgelegt. In der Praxis kommen diesbezüglich unterschiedliche Verrechnungsmethoden vor. Die Beiträge können an dem Flächenumfang einzelner Betriebe, dem Status der Beteiligten oder teilweise an beiden Faktoren orientiert werden. So wird hierzu als Maßstab in der Mehrheit der MR der Flächenumfang und Mitgliedsstatus herangezogen. In Bayern erfolgt die Aufteilung der Mitgliedsbetriebe i.d.R. nach folgenden Flächengrößen 5-20 ha, 20-50 ha und über 50 ha. In Ostdeutschland werden auch drei Flächenkategorien unterschieden und zwar bis 100 ha, 100-1.000 ha, über 1.000 ha. Nach dem Status werden die Mitglieder in drei Gruppen unterteilt: Landwirte, gewerbliche Mitglieder oder Lohnunternehmer sowie fördernde Mitglieder. Der jeweils fällige Beitrag wird in Abhängigkeit von der Zuordnung $\mathrm{zu}$ einer bestimmten Status- und Flächenumfangsgruppe bemessen. 
Die Verrechnung der Arbeitsleistung wird anhand von einer in jedem MR vorliegenden Preisliste vorgenommen. Die Preise für bestimmte Arbeiten werden bei Berücksichtigung der regionalen Relation von Angebot und Nachfrage in jedem einzelnen MR unterschiedlich gestaffelt. Die Abrechnung der Arbeitskosten erfolgt in der Praxis nach einem einheitlichen Muster. Jedes Mitglied, das eine bestimmte Leistung für einen anderen erbringt, hat am Ende der Arbeit einen Arbeitsbeleg auszufüllen, in dem er die nach der Aktivitätsart erfassten Leistungseinheiten in Stunden (Schlepper), Flächenleistung (Mähdrescher) oder Mengenleistung (Pressballen) einträgt. Der ausgefüllte Arbeitsbeleg wird dann vom Auftraggeber und vom Dienstleister unterschrieben und dem Geschäftsführer übermittelt. Die Ermittlung des Leistungsentgeltes erfolgt anhand einer Preisliste. Anschließend wird dem Arbeitnehmer der zustehende Betrag gutgeschrieben, das Konto des Auftraggebers wird mit dem gleichen Betrag belastet. Die prozentual erfasste, zusätzliche Vermittlungsgebühr, die bei jedem Fall erhoben wird, beträgt durchschnittlich $5 \%$ des Leistungsentgelts. Diese Gebühr wird je nach der örtlichen Regelung entweder von dem Auftragnehmer oder Auftraggeber oder u.U. von beiden zu gleichen Teilen entrichtet.

Der Wirkungsschwerpunkt eines Maschinenringes, der originär auf die Verbesserung der Wettbewerbsfähigkeit der landwirtschaftlichen Betriebe abzielte, geht zzt. weit über die klassische Kostensenkung und überbetriebliche Maschinenauslastung hinaus. Es wird heute ein immer größerer Wert der Arbeitsentlastung, dem Erschließen außerlandwirtschaftlicher Zuerwerbsmöglichkeiten sowie zusätzlichen Kosteneinsparungen durch z.B. gemeinsame Einkaufsaktivitäten beigemessen. Die Aufgaben, die zzt. in den MR realisiert werden, können grundsätzlich in fünf Gruppen unterteilt werden (siehe Übersicht 3-3).

Übersicht 3-3: Aufgabenbereiche der MR

\begin{tabular}{|lll|}
\hline \multicolumn{2}{|c|}{ Landtechnik: } & \multicolumn{1}{c|}{ Personal: } \\
\hline $\begin{array}{l}\text { - Maschinenvermittlung und -vermietung } \\
\text { - landtechnische Beratung }\end{array}$ & - Betriebshilfe \\
\hline Umweltservice & Handel: & Rohstoffveredlung \\
\hline -Bodenservice & - Knickbörse & - Rapsmethylesteranlagen \\
(z.B. Bodenprobendienst, Nährstoffbilanz & - Handelsgeschäfte & - Biogasgemeinschaften \\
Flächenvermessung) & - Stromeinkauf & \\
-Knick- und Forstarbeiten & - Solartechnik & \\
-Landschaftspflege & \\
\hline
\end{tabular}


In der ersten Gruppe befinden sich unverändert die Landtechnikaufgaben. Hierzu zählt außer der herkömmlichen Maschinenarbeitsvermittlung auch die Maschinenvermietung, die in Zusammenarbeit mit Maschinenhändlern erfolgt. Unter dieser Gruppe ist weiterhin die landtechnische Beratung zu subsumieren, die die einzelnen Geschäftsführer im Hinblick auf die regionale Relation von Angebot und Nachfrage vornehmen können.

Die zweite Gruppe der Maßnahmen betrifft das Personalpotenzial. Ein wichtiger Tätigkeitsbereich ist hier das Programm der Betriebshilfe, nach dem ein MR Betriebshelferarbeit vermittelt. Der Anfang der Initiative der Vermittlung der Betriebshelfer geht auf die siebziger Jahre zurück. In dieser Zeit hatte die Vermittlung von Betriebshelfern die Aufgabe, das Angebot von Alterskassen, kirchlichen Organisationen und Landwirtschaftskammern zu erweitern und gleichzeitig zur Bewältigung des Problems der Arbeitsspitzen oder notwendiger Vertretung der Landwirte im Fall von z.B. Krankheit oder Fortbildung beizutragen. Die Arbeitsvermittlung erfolgte vornehmlich durch den Einsatz von nebenberuflich tätigen Betriebshelfern, deren Bezahlung landwirtschaftliche Sozialversicherungsträger übernahmen. Wegen der geringen Kosten der so organisierten Betriebshilfe beschränkten sich die Aufgaben, zu deren Realisation Betriebshelfer in Anspruch genommen waren, nicht auf Notlagen oder saisonale Engpässe, sondern umfassten auch bestimmte Arbeiten im Haus- oder Waldbereich, die Bauhilfe sowie die Bedienung von betriebseigenen Maschinen. Hieraus etablierten sich in bestimmten Regionen MR, in denen im Zuge der weiteren Spezialisierung die herkömmliche Rolle der Betriebshelfer durch festangestellte Mitarbeiter übernommen wurde. Die Betriebshilfe gewann in den einzelnen Ländern einen recht unterschiedlichen Wert. Eine besondere Bedeutung erreichte die Betriebshelfervermittlung in Westfalen, wo im Jahr 1979 der Anteil dieser Aktivitäten rund $60 \%$ des gesamten Verrechnungswertes der MR ausmachte. Eine vergleichbar große Bedeutung kam der Betriebshelfervermittlung auch in Bayern zu. So wurde der Verrechnungswert der bayerischen MR im Jahr 1979 zu ca. $28 \%$ im Rahmen der Betriebshilfe generiert. Am schwächsten war der Tätigkeitsbereich in Schleswig - Holstein und Niedersachsen entwickelt, wo der Anteil der Betriebshilfe am gesamten Verrechnungswert generell nicht über $5 \%$ hinausging (vgl. ISERMEYER 1981, S.39). Derzeit ist die Betriebshilfe unverändert besonders stark in Bayern und in Baden - Württemberg verbreitet (siehe Tabelle 3-3). 
Tabelle 3-3: Organisation der Betriebshilfe in den MR im Jahr 2003

\begin{tabular}{|c|c|c|c|c|c|c|c|c|c|c|}
\hline $\begin{array}{l}\text { Gegenstand } \\
\text { der Nachweisung }\end{array}$ & 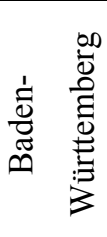 & 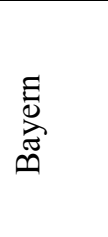 & 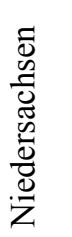 & 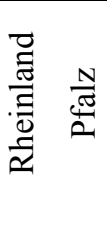 & 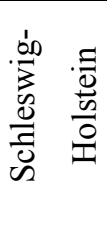 & $\begin{array}{l}\frac{\tilde{D}}{\tilde{T}} \\
\stackrel{\overrightarrow{0}}{0} \\
3\end{array}$ & $\begin{array}{l}\overline{0} \\
0 \\
0 \\
0 \\
\text { I }\end{array}$ & 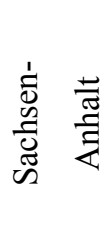 & 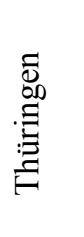 & 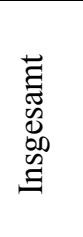 \\
\hline Verrechungswert in Mio. & 12,5 & 46,8 & 4,1 & 2,6 & 2,1 & 14,3 & 0,3 & 1,1 & 0,9 & 84,7 \\
\hline Hauptb. Betriebshelfer & 453 & 0 & 73 & 28 & 76 & 350 & 1 & 62 & - & - \\
\hline Nebenb. Betriebshelfer & 180 & 7724 & 453 & 420 & 17 & 234 & 14 & 73 & - & - \\
\hline Arbeitsstunden in 1000 & 685,6 & 4223,3 & 366 & 242,6 & 124,1 & 604,5 & 4,4 & 68,1 & 55,6 & 6374 \\
\hline davon soziale Betriebshilfe & 389,5 & 1645 & 147 & 114 & 74,6 & 472,2 & - & 65,3 & - & 2908 \\
\hline soziale Betriebshilfe in \% & 56,8 & 39 & 40 & 47 & 60 & 78 & - & 96 & - & 45,6 \\
\hline
\end{tabular}

Auf weitere Unterschiede in der Organisation der Betriebshilfe in bestimmten Bundesländern deutet auch die Verteilung der Arbeitszeit nach der Art der ausgeübten Tätigkeiten hin. Während in Sachsen - Anhalt ca. $96 \%$ und in Westfalen - Lippe nahezu $80 \%$ der in der Betriebshilfe geleisteten Arbeitstunden auf den Einsatz in sozialer Notlage entfallen, besitzt der Einsatz in den Zeitspitzen und für die Bewältigung gewöhnlicher Aufgaben in Bayern eine vorrangige Bedeutung.

Des Weiteren ist unter der Gruppe der Aufgaben aus dem Personalbereich der durch viele MR organisierte Zuerwerb für die Mitglieder zu subsumieren. Hierzu zählen z.B. Arbeiten im Winter- und Kehrdienst, Grünflächenpflege, Aufräumung und Durchforstung. Die Gruppe der Auftraggeber bilden u.a. Gebäudereinigungsunternehmen, Supermarktketten, Bahn oder Post. Weiterhin kommt den MR eine gewisse Bedeutung in der Beschäftigung von Langzeitarbeitslosen zu. Als Beispiel hierzu kann der MR - Mittelholstein dienen, in dem die Langzeitarbeitslosen im Bereich von Erhaltungs- und Pflegemaßnahmen in Landschaft und Wald beschäftigt werden. Die Initiative wird im Rahmen des SAM Projektes (Strukturanpassungsmaßnahmen) realisiert ${ }^{13}$.

Ein weiteres Betätigungsfeld heutiger Maschinenringe liegt im Bereich des Umweltservice. Unter Maßnahmen dieser Gruppe befinden sich z.B. komplexe Bodenanalysen (Bodenentnahme, Weiterleitung an Untersuchungszentren, Rücklauf der Proben mit

${ }^{13}$ In dem SAM (ehemals $\S 249 \mathrm{~h}$ des Arbeitsförderungsgesetzes), das mit der Beteiligung des europäischen Sozialfonds kofinanziert wird, kann die Bundesagentur (ehemalige Bundesanstalt) für Arbeit mit Lohnkostenzuschüssen für die Arbeitgeber vorübergehend bei der Beschäftigung von Arbeitslosen fördernd eingreifen. Die in diesem Programm erschlossenen Arbeiten sollen u.a. der Verbesserung der Umwelt, der sozialen Dienste, der Unterstützung der Behinderten, der Jugendhilfe, der Kulturarbeit und der Denkmalpflege dienen. Die Zielgruppe des Programms bilden v.a. die Langzeitarbeitslosen. 
Düngeempfehlungen), Flächenvermessung, Erstellung der Nährstoffbilanz aufgrund von Betriebsdaten sowie Verrichtung bestimmter Forst- und Landschaftspflegearbeiten.

Im Bereich der Handelsmaßnahmen, wird versucht, auf dem Wege der Interessenkopplung mehrerer Landwirte bestimmte Einkaufseinsparungen zu erzielen. Die Handelsgeschäfte umfassen z.B. den Bezug von Betriebstoffen (Diesel, Schmierstoffe und Heizöl), das Abschließen von Mobilfunkverträgen, die Anschaffung von bestimmten Maschinen und Autos, den Handel mit Knickholz oder den auf überregionaler Ebene durch eine Tochtergesellschaft BMR Service GmbH koordinierten Stromeinkauf.

Ein neues Betätigungsfeld, das in direktem Bezug auf umweltpolitische Maßnahmen etabliert wird, finden die MR in der Rohstoffveredlung. Die Entwicklung erfolgt auf bundesweiter Ebene und erfasst Aktivitäten, die die Potenziale einzelner MR überschreiten. Für diese Initiativen sind Gründungen von neuen Tochtergesellschaften signifikant, in denen die MR in Zusammenarbeit mit großen Umweltunternehmen als Gesellschafter auftreten. Als praktisches Beispiel hierzu können die Baargold $\mathrm{GmbH}$ in Baden-Württemberg oder die Marinabiodiesel GmbH \& Co. KG in Schleswig - Holstein angeführt werden. In beiden Fällen handelt es sich um die Rapsölherstellung und -verarbeitung.

Ein Rückblick auf die Entwicklung der MR bis hin zu ihrer gegenwärtigen Funktion veranschaulicht, dass die ursprünglichen Ziele der Organisation im Verlaufe der Zeit mit unterschiedlichem Erfolg realisiert wurden. Besondere Schwierigkeiten in den MR sind im Hinblick auf eine der wichtigsten Aufgaben, die erfolgreiche Einflussnahme auf den Umfang und die Art von Neu- und Ersatzanschaffung von Maschinen zu erkennen. Es ist nur teilweise gelungen das Grundziel, die Kosten zu senken und der bestehenden Übermechanisierung entgegen zu wirken, zu verwirklichen. $\mathrm{Zu}$ bedenken sind an dieser Stelle v.a. zwei Aspekte. Es handelt sich hier zum einen um Maschineninvestitionen, die in vielen landwirtschaftlichen Betrieben häufig unwirtschaftlich ohne Sicherung der erforderlichen Auslastung vorgenommen wurden und zum anderen um die von vielen Landwirten genutzte Möglichkeit auf schlagkräftigere Technik Dritter zurückzugreifen, wobei eigenbetriebliche Maschinen ungenutzt verblieben. Die auf diese Weise entstehende sog. Doppelmechanisierung hatte u.U. zur Folge, dass viele Betriebe andauernd in einer ungünstigen Kostenstruktur verharrten (vgl. GRIMM at all. 1993, S.2).

Im Hinblick auf die Funktionen, die die MR ursprünglich bei der Organisation des Maschineneinsatzes wahrnehmen sollen, ist eine konstante Bedeutungsabnahme der 
Nachbarschaftshilfe zu erkennen. Diese Tendenz ist, gemessen an den Anteilen der Maschinenarbeitsvermittlung an den gesamten Verrechnungswerten, von MR in Nordrhein Westfalen abgesehen, in allen Bundesländern zu verzeichnen. Besonders prägnant ist der Vorgang in Regionen mit größeren Betrieben, wie Schleswig - Holstein und im nördlichen Teil Niedersachsens. Die Rolle von vielen MR dieser Regionen konzentriert sich auf die Vermittlung der Dienstleistungen der LU. Es wird geschätzt, dass die Verrechnungswerte der MR in einigen Regionen bis zu $90 \%$ durch die Vermittlung der LU- Arbeiten generiert werden (Z.B. Traulsen 2004, PentZlin 2004).

Die Maschinenarbeitsvermittlung scheint eine relativ große Bedeutung in den bayerischen MR zu haben. Werden aber zur weiteren Analyse der Bedeutung der Maschinenarbeitsvermittlung die Daten zur Aufteilung der Arbeitszeit der Mitarbeiter von repräsentativen bayerischen MR herangezogen, so zeigt sich, dass für die reine Maschinenarbeitsvermittlung lediglich $7 \%$ der Arbeitszeit beansprucht werden. Ungefähr $21 \%$ der Arbeitszeit in den MR werden der Organisation der Betriebshilfe gewidmet. Die Hälfte der Arbeitszeit entfällt auf solche Aktivitäten wie Handel in den oben angesprochenen Bereichen, Organisation des Zuerwerbs für Mitglieder oder Vereinsarbeit (siehe Abbildung 3-2).

Abbildung 3-2: Anteile der Tätigkeitsbereiche an der gesamten Arbeitszeit der Mitarbeiter (inkl. Geschäftsführer) in den bayerischen MR

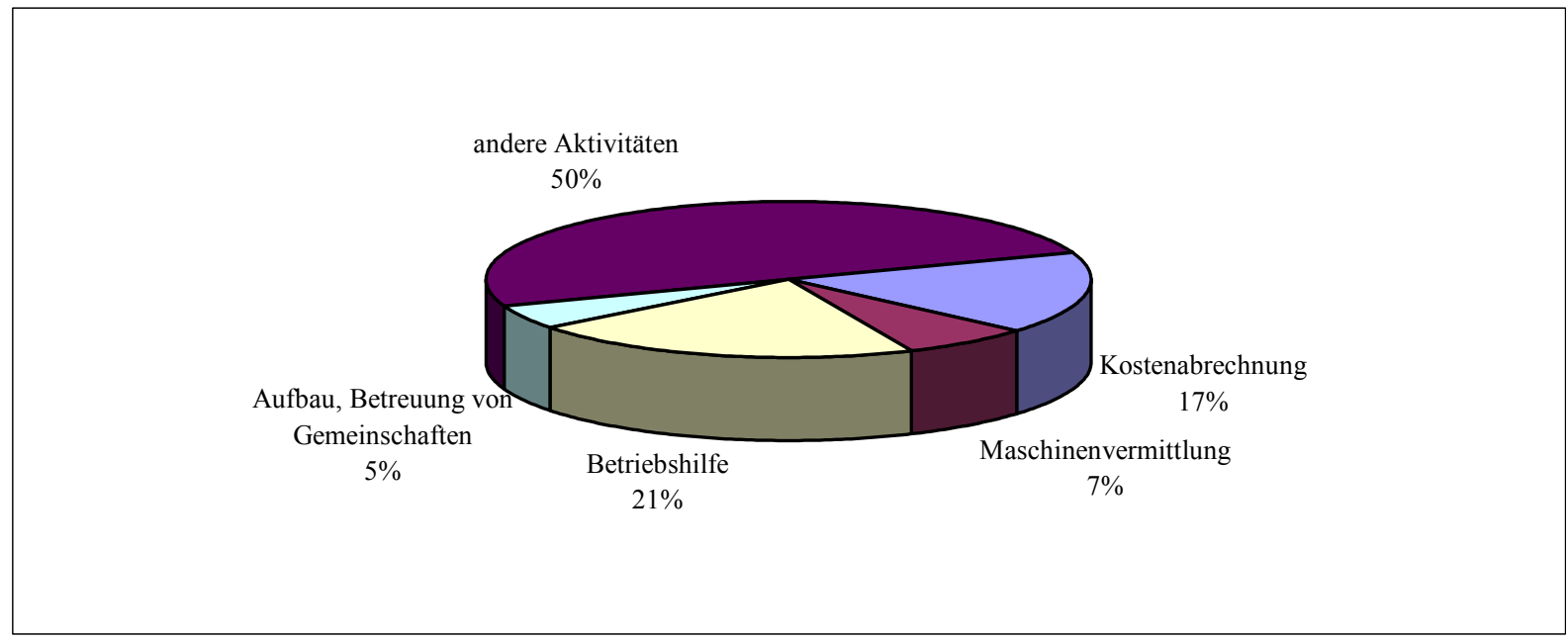

Quelle: Pellot KBM e.V. 2003

Eine weitere Analyse der statistischen Daten aus den MR in Bayern zeigt, dass das Ziel, außer potenziellen Auftraggebern einen möglichst breiten Kreis von Auftragnehmern zu erschließen, auf Dauer nicht erreicht wurde (siehe Tabelle 3-4). Die Gegenüberstellung der Daten aus dem Jahr 1992 mit denen aus dem Jahr 2004 zeigt zwar eine positive 
Entwicklungstendenz in der Zunahme der Anzahl der Auftraggeber. Werden jedoch die Zahlen unter Berücksichtigung anderer Aktivitäten wie z.B. die oben angesprochene Betriebshelfervermittlung analysiert, so wird deutlich, dass die Anzahl der Betriebe, in denen die Maschinenarbeiten im Rahmen eines Maschinenringes organisiert werden, relativ gering ist. Weiterhin nimmt die Anzahl der gleichzeitig als Auftragnehmer und Auftraggeber auftretenden Landwirte zugunsten der inaktiven Mitglieder deutlich ab.

Tabelle 3-4: Prozentualer Anteil der Auftraggeber und Auftragnehmer in den bayerischen Maschinenringen in den Jahren 1992- 2005 in \%

\begin{tabular}{|l|c|c|c|c|}
\hline \multicolumn{1}{|c|}{ Jahre } & 1992 & 2002 & 2004 & 2005 \\
\hline ausschließlich Auftraggeber & 29,6 & 37,3 & 38,4 & 37 \\
\hline ausschließlich Auftragnehmer & 6,3 & 6,8 & 7,5 & 6,5 \\
\hline Gleichzeitig Auftraggeber und -nehmer & 40,4 & 27,2 & 26,6 & 26 \\
\hline inaktive Mitglieder & 23,7 & 28,7 & 27,5 & 30,5 \\
\hline
\end{tabular}

Quelle: Walter, KBM e.V. 2005

Bestimmte Erfolge in der Maschinenarbeitserledigung sind für solche MR kennzeichnend, die die Nutzung leistungsfähiger und besserer als in der Mehrheit der landwirtschaftlichen Betriebe vorhandener Technik ermöglichten. Gegenwärtig spielen die MR eine wesentliche Rolle bei komplizierten und aufwendigen Verfahren, die die einzelbetrieblichen Kapazitäten übersteigen und in der Praxis häufig das Entstehen von ganzen Arbeitsketten erfordern. Bei relativ einfachen Arbeiten wie z.B. Heuernte, Säen oder Pflügen ist die Rolle der MR generell eher unbedeutend (TRAULSEN 2004).

Den MR kommt heute eine wesentliche Bedeutung als Koordinationszentren der Interessenbindung einzelner Landwirte zu. Aufgrund regional bestehender Nachfrage nach bestimmten Maschinenarbeiten kommt es zur Gründung zahlreicher Gemeinschaften, wo der Maschineneinsatz durch einen MR organisiert und abgerechnet wird. Ein Beispiel solcher Initiative stellt der Zusammenschluss von Landwirten dar, die mit organisatorischer Beteiligung eines MR eine GbR für Zuckerrübenernte und -transport gründen.

Zusammenfassend kann festgestellt werden, dass die MR heutzutage in der deutschen Landwirtschaft nach wie vor eine wesentliche Rolle spielen. Dies betrifft allerdings im konstant abnehmenden Ausmaß die ursprüngliche Aufgabe der Maschinenarbeitsvermittlung. Eine größe Bedeutung in der durch die MR organisierten Maschinearbeitserledigung spielen die Lohnunternehmen. In den MR handelt es sich derzeit häufig vielmehr um die Bindung der Kapazitäten der einzelnen Mitglieder mit dem Zweck, neue, häufig außerlandwirtschaftliche 
Arbeitsbereiche zu erschließen oder bestimmte Einsparungspotenziale zu aktivieren. Hierzu zählen Tätigkeiten im Handels- und Rohstoffveredlungsbereich, Organisation von Arbeiten im Kommunalbereich oder Pflege- und Sanierungsmaßnahmen. In diesem Zusammenhang kommt es zur Gründung von verschiedenen Tochtergesellschaften, in denen die MR über die in der Form des e.V. auferlegten Grenzen hinaus ihre Position als selbständiges landwirtschaftsnahes Unternehmen, meist als $\mathrm{GmbH}$, zu sichern versuchen.

\subsubsection{Bruchteilsgemeinschaft und Maschinengesellschaft}

Eine wesentliche Bedeutung in der Organisation des überbetrieblichen Maschineneinsatzes kommt in der deutschen Landwirtschaft den Maschinengemeinschaften zu. Im Hinblick auf die Anzahl der Beteiligten und der eingesetzten Maschinen sowie des Arbeitsumfangs wird in der Praxis zwischen Klein- und Großgemeinschaften unterschieden. Im ersten Fall handelt es sich i.d.R. um Zusammenschlüsse von 2 bis 4 Landwirten, die sich zur gemeinsamen Anschaffung und Nutzung bestimmter Maschinen vereinigen. Die Wirkung einer solchen Gemeinschaft bleibt im Regelfall auf den Arbeitsbedarf in den Mitgliedsbetrieben beschränkt. Ein eventueller Maschineneinsatz bei Dritten spielt hier nur eine Ergänzungsrolle. In den Großgemeinschaften, die entweder in GbR oder in $\mathrm{GmbH}$ organisiert werden, sind sowohl der Umfang der erbrachten Arbeiten als auch die Mitgliederanzahl i.d.R. größer als im Fall der ersten Form. Entsprechend der Struktur der Mitgliedsbetriebe und der daraus resultierenden Erfordernisse kommen in den Maschinengemeinschaften unterschiedliche Vorteile zum Tragen. Im Wesentlichen handelt es sich hier um die Reduktion der Fremdkapitalbelastung bei Investitionen und die Steigerung der Maschinenauslastung.

Die Entwicklungsphase der Maschinengemeinschaften begann in den fünfziger und der ersten Hälfte der sechziger Jahre. Die dynamische Verbreitung dieser Kooperationsform steht in einem engen Zusammenhang mit dem Übergang von der überwiegenden Handarbeit zur Vollmechanisierung. Dieser Vorgang, dies betrifft insbesondere die südlichen Regionen, erfolgte unter Bedingungen einer vorherrschenden ungünstigen Größenstruktur und einer generell niedrigen Kapitalausstattung der Betriebe. Der wachsenden Nachfrage nach Fremdmaschinenarbeit stand ein relativ karges Angebot gegenüber. Die Maschinengemeinschaften galten damals als besonders geeignetes Mittel, den überwiegend kleinen Betrieben den Anschluss an die Mechanisierung zu ermöglichen. Einen wesentlichen Beitrag zur Entwicklung dieser Form der Maschinennutzung leistete die Förderungspolitik der Bundesregierung (vgl. ISERMEYER 1981, S. 34). Die finanzielle Unterstützung der Maschinengemeinschaften, die von der Seite des Bundes in den Jahren 
1951-1965 erfolgte, umfasste v.a. die Zinsverbilligung für Maschinenkredite sowie die Finanzierung der Ausbildung für Fachberater. Die Förderung erfolgte in zwei separaten Stufen. Die ersten Maßnahmen wurden im Rahmen des Projekts der „Förderung der Bildung von Schlepper- und Mähdreschergemeinschaften“ in den Jahren 1951-1954 getroffen. Die Genehmigung der Fördermittel war von der Erfüllung bestimmter Voraussetzungen abhängig. Bei den Schleppergemeinschaften sollte die Anzahl der Mitglieder zwischen 2 und 6 liegen, wobei die gesamte von den Beteiligten bewirtschaftete LF insgesamt 60 ha nicht übersteigen durfte. Bei den Mähdreschergemeinschaften konnte die Mähdruschfläche von 30 bis 70 ha reichen (vgl. CRÖNLEIN 1979, S. 64). Mit den damals als verlorene Zuschüsse und Zinsverbilligungen verausgabten Mitteln wurden Kredite für Maschineninvestitionen von insgesamt 6,6 Mio. DM verbilligt (vgl. IsERMEYER 1967, S. 694). Die zweite Phase der Förderung der Maschinengemeinschaften erfolgte in den Jahren 1954-1965 im Rahmen der sog. Maßnahmen zur „Beschaffung von Gemeinschaftseinrichtungen und Gemeinschaftsmaschinen“. Ähnlich wie beim ersten Programm bildeten hier die Zielgruppe die relativ kleinen und mittleren Betriebe. Die Summe der verausgabten Fördermittel, die am Anfang des Programms (1954-1959) ca. 7 Mio. DM betrug, wurde in den nächsten Jahren mehr als verdreifacht (vgl. CRÖNLEIN 1979, S. 67).

Im Jahr 1960 wurde die Zahl der Maschinengemeinschaften auf dem gesamten Bundesgebiet auf ungefähr 41.000-45.000 geschätzt. Mit deutlichem Abstand entfielen die meisten Gemeinschaften auf die südlichen Bundesländer wie Baden - Württemberg, Hessen und Rheinland - Pfalz (vgl. CRÖNLEIN 1980, S. 178). Mit ungefähr 162.000 Beteiligten umfassten die Maschinengemeinschaften ca. $11 \%$ aller landwirtschaftlichen Betriebe. Mit der Einstellung der Förderung seit dem Jahr 1965 (mit Ausnahme von Hessen) ist ein allmählicher Rückgang der Anzahl der Zusammenschlüsse zu verzeichnen. So gab es im Jahr 1980 ungefähr 21.000 Maschinengemeinschaften (siehe Tabelle 3-5). Folgerichtig ging im Vergleich zu dem Jahr 1960 auch die Anzahl der in den Maschinengemeinschaften aktiven Landwirte zurück. Nach wie vor blieben aber ca. $11 \%$ aller zu dieser Zeit existierenden landwirtschaftlichen Betriebe in den Maschinengemeinschaften organisiert (vgl. ISERMEYER 1981, S.36). 
Tabelle 3-5: Entwicklung der Maschinengemeinschaften in der Bundesrepublik Deutschland in den Jahren 1960-1980

\begin{tabular}{|c|c|c|c|c|c|}
\hline & 1960 & 1970 & 1973 & 1977 & 1980 \\
\hline Anzahl der MG & 41000 & 40000 & 35000 & 29000 & 21000 \\
\hline Mitglieder der MG & 162000 & 130000 & 100000 & 90000 & 87000 \\
\hline
\end{tabular}

In der analysierten Zeitspanne 1960-1980 ist eine permanente Abnahme der Anzahl der Großgemeinschaften zu erkennen. Diesem Geschehen steht eine wachsende Bedeutung der Kleingemeinschaften gegenüber. Dies lässt sich teilweise durch die Vorteilhaftigkeit der kleinen gegenüber den großen Gemeinschaften erklären. Nicht zuletzt hängt der Rückgang der Anzahl der Maschinengemeinschaften mit der erfolgreichen Entwicklung anderer Formen der überbetrieblichen Maschinennutzung, insbesondere der MR zusammen (vgl. ISERMEYER 1981, S. 34).

Eine vollständige Datenerhebung zur Weiterentwicklung der Maschinengemeinschaften wurde nach der geschilderten Zeitspanne nicht erneut vorgenommen. Es liegen zu der Verbreitung der Maschinengemeinschaften aktuell keine exakten Zahlen vor. Aussagen zu der gegenwärtigen Bedeutung dieser Form überbetrieblicher Maschinennutzung können lediglich anhand allgemeiner Schätzungen getroffen werden.

Die kleineren Maschinengemeinschaften haben eine besondere Bedeutung in den Ländern, in denen verhältnismäßig kleinen Betriebe vorherrschen. Dies betrifft insbesondere Baden Württemberg, Bayern und Hessen. Die Gemeinschaften werden i.d.R. gegründet, um bestimmte Maschinen einzusetzen, die einzelbetrieblich nicht ausgelastet werden könnten. Hierzu zählen z.B. Mähdrescher, Futtermischwagen, Pflanzenschutzspritze und Güllefass. Vor diesem Hintergrund kann auch die Entwicklung der Maschinengemeinschaften in den östlichen Bundesländern betrachtet werden. Im Gegenteil zu den westlichen Regionen ist hier diese Form der Zusammenarbeit bei dem Einsatz der o.g. Maschinen wenig verbreitet. Eine Ausnahme bilden in den östlichen Bundesländern Maschinengemeinschaften für die Organisation der Zuckerrübenernte. Es handelt sich dabei um Einheiten, die nach dem Vorbild der westlichen Zusammenschlüsse mehrere Landwirte vereinigen und in denen schlagkräftige sechsreihige Zuckerrübenroder eingesetzt werden (Mann 2006).

Das Gelingen einer Maschinengemeinschaft ist von zahlreichen Faktoren abhängig. Einen der wichtigsten Problembereiche bildet die oben angedeutete Betriebsstruktur. Es ist von großer Bedeutung, dass die Betriebe der kooperierenden Landwirte eine ähnliche Produktionsstruktur 
aufweisen. Gleichzeitig aber soll der Einsatz bestimmter Maschinen zu unterschiedlichen Zeitpunkten ermöglicht werden. Eine wesentliche Rolle spielt hier u.a. die Aufteilung der einzelnen Feldschläge. Bei kleinen Parzellen kann eine große schlagkräftige Maschine meistens nicht effizient eingesetzt werden. Andererseits nimmt mit der erforderlichen Steigerung des Umfangs der LF, die im Rahmen einer Maschinengemeinschaft bewirtschaftet werden, das Risiko der Verspätung in der Arbeitsleistung deutlich zu. In der Praxis kann die Verteilung der Einsatzzeit einer gemeinsamen Maschine zu Schwierigkeiten führen. Eine weitaus größere Bedeutung als in anderen Kooperationsformen besitzen in den Maschinengemeinschaften die sozialen Aspekte der Zusammenarbeit. Daher ist es von vorrangiger Bedeutung, dass die Kooperationspartner bestimmte interpersonelle Fähigkeiten aufbringen. Ein erfolgreiches Bestehen einer Maschinengemeinschaft ist häufig stärker von dem sozialen Umgang der Partner miteinander als von ökonomischen Überlegungen abhängig. Die Missverständnisse die auf dem sozialen Grund entstehen, sind auch häufig die Ursache für das Scheitern der kleineren Gemeinschaften (vgl. z.B. WÜST 2003, S. 8, GRIMM 1995, S. 240).

Des Weiteren wird in der Praxis ein Trend beobachtet, im Rahmen einer Maschinengemeinschaft ganze Arbeitsketten beispielsweise beim Silieren, für Bodenbearbeitung, Bestellung, Ernte und den Transport von Zuckerrüben zu gestalten (vgl. LiNK 1995, S. 31). Der in dieser Form organisierte Maschineneinsatz geht häufig über den Rahmen der Landwirtschaft zum Gewerbebereich hinaus ${ }^{14}$. Ein praktisches Beispiel hierzu stellen die angesprochenen Zusammenschlüsse mehrerer Landwirte für die Organisation von Zuckerrübenernte und -transport dar. Gemeinschaften dieser Art werden häufig unter organisatorischer Betreuung von Maschinenringen geführt. Zum Einsatz kommen dabei in der Regel selbstfahrende Zuckerrübenroder. Die Maschinen werden nicht von jedem einzelnen Landwirt, sondern nur von bestimmtem Personal bedient. Die gesamte Anbaufläche solcher Gemeinschaften erstreckt sich i.d.R. von ca. 500 ha bis zu über 1.000 ha. Neben Gemeinschaften, die aus einer relativ kleinen Gruppe von Landwirten mit großen Anbauflächen bestehen, sind in der Praxis Zusammenschlüsse von mehreren Landwirten bekannt, bei denen die durchschnittliche Flächengroße des Rübenanbaus oft unter 2 ha liegt. Die Rolle der MR bei einer solchen Gemeinschaft besteht in der Organisation des Maschineneinsatzes bei der Rübenkampagne, in der Kostenabrechnung und der Buchführung.

\footnotetext{
${ }^{14}$ Der außerbetriebliche Maschineneinsatz gilt so lange als landwirtschaftliche Tätigkeit, wie die Einnahmen daraus nicht mehr als 51.500 EUR oder ein Drittel des Gesamtumsatzes des Betriebes pro Jahr übersteigen. Falls die Maschinen im nichtlandwirtschaftlichen Bereich eingesetzt werden (z.B. Kommunalarbeiten) dürfen die Betriebseinnahmen 10.300 EUR pro Jahr nicht übersteigen (vgl. EStR 15.5 Abs.10).
} 
Die Planung des Maschineneinsatzes erfolgt i.d.R. einige Wochen vor der Rübenernte anhand von Informationen zu der Struktur der Rübenschläge, die von jedem einzelnen Landwirt in einem Formblatt angegeben werden. Die Struktur der Anbauschläge der einzelnen Mitglieder wird meistens mithilfe eines Computerprogramms hinterlegt und nach Bedarf z.B. in jährlichen Abständen auf den aktuellen Stand gebracht. Im Vorfeld der Kampagne erhält jeder Beteiligte eine Liste mit den entsprechenden Terminen für das Rübenroden und die -abfuhr. Die Abfuhrtermine werden durch einen MR mit der Zuckerrübenfabrik vereinbart. Die Kostenabrechung des Maschineneinsatzes erfolgt aufgrund der tatsächlich erbrachten Arbeitsleistung auf den Flächen eines jeden Mitglieds bei Berücksichtigung seiner bei Gründung der Gemeinschaft eingebrachten Anteile. Die evtl. erwirtschafteten Überschüsse werden, nach einem im Vorfeld festgelegten Verteilungsschlüssel an die einzelnen Mitglieder ausgezahlt.

In großen Maschinengemeinschaften kann dank Interessenbindung vieler Landwirte der Kapitalbedarf für Maschineninvestitionen je Mitglied beachtlich gesenkt werden. Dies hat eine besondere Bedeutung bei der Anschaffung großer schlagkräftiger Aggregate, deren Einsatz in Eigenmechanisierung oder sogar im Rahmen von kleineren Maschinengemeinschaften wirtschaftlich nicht effizient erfolgen könnte. Bei der Überlassung der tatsächlichen Führung einer solchen Maschinengemeinschaft an dritte Personen, mit Vorbehalt der Stimmrechte der Mitglieder, können die potenziellen Probleme sozialer Natur, die die Zusammenarbeit vieler Mitglieder mit sich bringt, relativ leicht umgangen werden.

\subsubsection{Lohnunternehmen}

Eine weitere Form des überbetrieblichen Maschineneinsatzes, die in der deutschen Landwirtschaft eine wesentliche Stellung einnimmt, ist die Zusammenarbeit mit den Lohnunternehmen. Es handelt sich in diesem Fall um die Inanspruchnahme der Dienstleistungen von Unternehmen, die ihre eigenen Maschinen i.d.R. mit eigenem Bedienungspersonal zu einem bestimmten Preis dem Auftraggeber zur Verfügung stellen. Im Gegensatz zu anderen Kooperationsformen mit höherer Bindungsintensität bleibt die Entscheidungsfreiheit der einzelnen landwirtschaftlichen Betriebe in Zusammenarbeit mit den LU unberührt. Zu weiteren spezifischen Vorteilen, die in Zusammenarbeit mit den LU zum Tragen kommen können, gehören:

- $\quad$ Abbau von Arbeitsspitzen, Sicherung der Termingerechtheit,

- $\quad$ Nutzen vom Fachwissen der Lohnunternehmen, 
- $\quad$ Einsatz von moderner schlagkräftiger Technik, dank derer die Ordnungsmäßigkeit und Qualität der ausgerichteten Tätigkeiten gesichert werden,

- $\quad$ Erhöhung der Betriebsflexibilität durch Freisetzung von liquiden Finanzmitteln,

- $\quad$ Verringerung des Risikos von Fehlinvestitionen.

Eine systematische Abgrenzung der in der Praxis vertretenen Formen des überbetrieblichen Lohnmaschineneinsatzes kann allgemein wie folgt dargestellt werden:

1. Landwirtschaftliche Lohnunternehmen, in denen Einzelpersonen neben der Hauptbeschäftigung in eigenen landwirtschaftlichen Betrieben im Rahmen von freien Kapazitäten bestimmte Lohmaschinenarbeiten anbieten.

2. Gewerbliche Lohnunternehmen, in denen Einzelpersonen oder Gesellschaften den Lohnmaschineneinsatz als alleinige oder überwiegende Tätigkeit betreiben.

3. Lohnmaschinenhaltung von Kommunen, Verbänden (Wasser- und Bodenverbände), öffentlicher Hand (Maschinenstationen) sowie von landwirtschaftlichen Genossenschaften.

Der Lohnmaschineneinsatz erfolgte ursprünglich in der ersten der o.g. Formen, also im Nebenerwerb der überdurchschnittlich mechanisierten Betriebe, die ihre Maschinen im Nachbarkreis einsetzten. Die auf dieser Basis angebotene Maschinendienstleistung entwickelte sich unter bestimmten Bedingungen $\mathrm{zu}$ einer häufig über die Grenzen der landwirtschaftlichen Tätigkeit hinausgehenden, gewerblichen hauptberuflichen Tätigkeit. Die dritte genannte Form spielte zwar in der Entwicklungsphase des Lohnmaschineneinsatzes in bestimmten Regionen eine wichtige Rolle. Ihre Bedeutung nahm jedoch danach wesentlich ab, so dass sie heute nur selten anzutreffen ist (Schmidt 2004).

Die Entwicklung des Lohnmaschineneinsatzes nahm vor dem Hintergrund der regionalen Differenzen der landwirtschaftlichen Struktur einen unterschiedlichen Charakter an. Besonders günstige Voraussetzungen fanden die LU in Bereichen, in denen Spezialmaschinen benötigt wurden. Dies betrifft v.a. große Betriebe, in denen wegen wachsender Spezialisierung im Veredlungsbereich oder Vorhandensein günstiger außerlandwirtschaftlicher Erwerbsmöglichkeiten bestimmte Arbeiten an Dritte ausgelagert wurden. Vor diesem Hintergrund entwickelten sich die LU besonders stark in den nord- und nordwestlichen Ländern wie Schleswig - Holstein, dem nördlichen Teil Niedersachsens sowie Nordrhein-Westfalen. Fördernd auf die Entwicklung dieser Form der Maschinenarbeitserledigung wirkte hier die große Anzahl von Veredlungsbetrieben. In 
Nordrhein- Westfalen kommt die mit der direkten Nähe der Industriegebiete verbundene Möglichkeit der Aufnahme einer außerlandwirtschaftlichen Erwerbstätigkeit hinzu. Der Eigenmechanisierung stehen unter diesen Bedingungen, insbesondere infolge teurer Arbeitskraft sowie der Konzentration auf kapitalaufwendige Produktionsbereiche, hohe Opportunitätskosten gegenüber. Die Nachfrage nach Fremdmaschinenarbeit, die durch Erschließen der freien Teilkapazitäten im Rahmen der Nachbarschaftshilfe oder gelegentlicher Dienstleistung nicht befriedigt werden konnte, förderte verstärkt die Entwicklung der hauptberuflichen LU in diesen Regionen (vgl. RiEMANN 1980, S. 32). In Gebieten, in denen verhältnismäßig kleine Betriebe vorherrschen und die Maschinenarbeiten vornehmlich in Eigenmechanisierung oder im Rahmen der Nachbarschaftshilfe organisiert wurden, beschränkte sich der Lohnmaschineneinsatz längere Zeit auf gelegentliche Dienstleistung von landwirtschaftlichen Betrieben, die ihre Maschinenkapazitäten oft im Rahmen eines MR zusätzlich auslasteten. Die hauptberuflichen professionellen LU waren in diesen Regionen relativ selten. Als Indiz unterschiedlicher Entwicklung kann hier die Ausstattung der LU bestimmter Regionen dienen. Während im Jahr 1979 in Norddeutschland die Hälfte aller LU verschiedene Maschinen mit dem Anschaffungswert zwischen 500.000 und 1 Mio. DM besaß, verfügte in Süddeutschland nur ein Fünftel der Lohnunternehmer über Maschinen ähnlicher Größe. Über $40 \%$ der LU der südlichen Regionen besaßen Maschinen deren Wert nicht über 40.000 DM hinausging (vgl. SCHMIDT 1983, S.52).

Die angesprochenen Unterschiede in der Entwicklungsstruktur der LU in den einzelnen Ländern sind immer noch existent. Nach wie vor gibt es neben professionellen LU solche, die von eigener landwirtschaftlicher Produktion ausgehend ihre Maschinen in dritten Betrieben verhältnismäßig selten einsetzen. In diesem Fall ist es häufig nicht das Ziel, das Angebot langfristig zu erweitern, sondern vielmehr eine höhere Auslastung von Maschinen und/oder eigenen Arbeitskapazitäten zu erzielen. Die Dienstleistung in dieser Ausprägung geht i.d.R. nicht über die Grenzen der landwirtschaftlichen Tätigkeit hinaus.

Die Verbreitung der LU ist trotz großer praktischer Bedeutung dieser Einrichtungen zahlenmäßig nicht zu erfassen. Es liegt kein vollständiges Datenmaterial vor, auf das man bei der Analyse der Verbreitung des Lohnmaschineneinsatzes zurückgreifen könnte. Laut Schätzungen gibt es in ganz Deutschland ungefähr 3.000 professionelle LU (SCHMIDT 2004). 
Die LU spielen zzt. in der ÜMV eine wichtige Rolle. Die Zusammenarbeit mit den LU entwickelt sich als logische Konsequenz des technischen Fortschritts und der Strukturentwicklung insbesondere in den Regionen mit einer hohen Konzentration von Veredlungsbetrieben. In diesen Fällen sind häufig nicht direkt die Mechanisierungskosten ausschlaggebend, sondern vielmehr die Möglichkeit die bei der Arbeitsauslagerung freigesetzten Faktoren anderweitig günstiger zu verwenden. Es handelt sich hier v.a um die Freistellung von Zeit- und Finanzkapazitäten für eine weitere Betriebsentwicklung oder das Nachgehen einer außerlandwirtschaftlichen Tätigkeit (KowALEWSKI 2004). So sind die LU nach wie vor besonders stark in den nordwestlichen Regionen vertreten. Allein in Niedersachsen, Schleswig Holstein und Nordrhein - Westfalen sind ungefähr $60 \%$ aller LU ansässig (vgl. BLU e.V. 2002). Mit deutlichem Abstand stellen die LU in diesen Regionen die wichtigste Form der ÜMV dar. Der Anteil der LU an den in allen Formen der ÜMV erbrachten Leistungen wird auf über $80 \%$ geschätzt (SCHMIDT 2004). Die LU werden verstärkt bei Vorgängen in Anspruch genommen, in denen spezielle schlagkräftige Maschinen zur Anwendung kommen. $\mathrm{Zu}$ den häufigsten Arbeiten zu denen die LU herangezogen werden, gehört Mähdrusch, Gülleausbringung, Silagebereitung, Pflanzenschutz, Zuckerrübenernte, Transport der landwirtschaftlichen Erzeugnisse und Futterverteilung mit einem Futtermischwagen. Neben den LU, die ihre Dienste nur in bestimmten Bereichen anbieten, sind in diesen Regionen zahlreiche LU vertreten, die das gesamte Spektrum der Arbeiten bis hin zur kompletten Betriebsbewirtschaftung erschließen. Die Bedeutung dieser Form der überbetrieblichen Maschinennutzung in diesen Regionen kann außer der angesprochenen strukturellen Voraussetzungen auch durch die historisch gewachsene Bereitschaft der Betriebsleiter, partielle Unternehmensfunktionen aus dem Betrieb auszugliedern, begründet werden (vgl. SCHMIDT 1983, S. 52).

In Regionen, in denen relativ kleine Ackerbau- oder Gemischtbetriebe vorherrschen und gleichzeitig keine Möglichkeiten gegeben sind, eigene Arbeitskraft außerhalb der Landwirtschaft günstig einzusetzen, sind die professionellen LU zzt. relativ selten vertreten. Die Lohnmaschinenleistung wird in diesen Regionen i.d.R. durch landwirtschaftliche Betriebe angeboten und geht generell nicht über den Einsatz der einzelnen Maschinen hinaus.

Zahlenmäßig sind die LU auch in den östlichen Bundesländern wenig verbreitet. Auf diese Regionen entfallen nach allgemeinen Schätzungen ca. 200 LU (Schmidt 2006). Unter Bedingungen, in denen große schlagkräftige Maschinen im Rahmen der Eigenmechanisierung effizient eingesetzt werden können, sind die Entwicklungschancen der LU eingeengt. Es 
bestehen hier allerdings einige spezialisierte LU, die mit den Leistungskapazitäten die Größen vieler LU der westlichen Bundesländer übersteigen. Diese LU sind aufgrund der günstigen Flächenstrukturen der landwirtschaftlichen Betriebe dieser Regionen in der Lage die Dienstleistungen besonders günstig anzubieten. Zu den typischen Beispielen der Arbeiten, in den die LU in Anspruch genommen werden, zählen die Gras- und Maissilagebereitung, die Gülleausbringung oder die Zuckerrübenernte (Mann 2006).

\subsection{Zusammenfassung}

Die vorangegangenen Erörterungen veranschaulichen einen vielfältigen Zusammenhang zwischen dem gesamten Wandel in der Landwirtschaft Deutschlands und der Entwicklung der überbetrieblichen Maschinennutzung. Von Bedeutung sind an dieser Stelle regionale Strukturunterschiede und die daraus resultierenden Ausgangsbedingungen. In den süddeutschen Regionen, in denen ungünstige Kleinstrukturen vorherrschten, erfolgte der überbetriebliche Maschineneinsatz ursprünglich vornehmlich auf dem Wege der Nachbarschaftshilfe. Eine zunehmende Bedeutung dieser Form der Zusammenarbeit findet den Ausdruck in einer starken Verbreitung der MR. Die ursprüngliche Aufgabe der MR war die Optimierung des Maschineneinsatzes unterschiedlich ausgestatteter Betriebe zu fördern. In den kleineren Betrieben bildete die überbetriebliche Zusammenarbeit oft eine Voraussetzung für weitere Entwicklung der Mechanisierung. In den größeren Betrieben, die als Leistungsanbieter agierten, war der überbetriebliche Maschineneinsatz mit der Steigerung der Maschinenauslastung und der dadurch erzielbaren Kostensenkung verbunden. Des Weiteren kam in diesen Regionen auch eine Bedeutung den Maschinengemeinschaften zu. Dabei handelte es sich vornehmlich um die Zusammenarbeit von mittleren Betrieben, die bestimmte, meistens Spezialmaschinen, gemeinsam einsetzten. Einen anderen Charakter hatte die Entwicklung in den nördlichen Regionen. Schon geschichtlich bedingt wurden hier viele Maschinenarbeiten durch LU verrichtet. Die von vielen Betrieben dieser Region angestrebte innerbetriebliche Entwicklung und die damit verbundene Bereitschaft bestimmte Maschinenarbeiten auszulagern, förderte dauerhaft die Verbreitung von professionellen, gewerblichen LU. Darüber hinaus entwickelten sich in Deutschland, als eine Alternative für die Eigenmechanisierung oder Fremdmaschineneinsatz, auch die Maschinengemeinschaften. Dies betrifft insbesondere mittlere ackerbaulich orientierte Betriebe, in denen die Zusammenarbeit im Rahmen einer Maschinengemeinschaft eine bessere Verwendung der vorhandenen Produktionsfaktoren ermöglichte. 
Die verschiedenen Formen der überbetrieblichen Zusammenarbeit von Nachbarschaftshilfe über Maschinengemeinschaften bis zur Inanspruchnahme von Fremdmaschinenleistung haben einen unterschiedlichen Arbeits- und Kapitalbedarf. Daraus ergeben sich unterschiedliche Auswirkungen auf die Betriebsstruktur und weitere Entwicklungswege. In der Gruppe der kleineren und mittleren wachstumorientierten Betriebe eröffnete die Zusammenarbeit, sei es in Nachbarschaftshilfe oder in einer Maschinengemeinschaft, zunächst die Möglichkeit die knappen Produktionsfaktoren besser auszunutzen und dadurch eine Kostensenkung zu erzielen. Unter bestimmten Bedingungen verlagerte sich der Schwerpunkt der in einer Zusammenarbeit angestrebten Vorteile häufig von der Kostenreduzierung auf weitere betriebsstrukturelle Effekte. Diese waren umso größer, je effizienter die freigesetzten Faktoren in Form von Kapital und Arbeit anderweitig eingesetzt werden konnten.

Die überbetriebliche Zusammenarbeit förderte die Arbeitsteilung zwischen unterschiedlich strukturierten Betrieben und ermöglichte eine optimale Betriebsentwicklung durch die Aufoder Abstockung der Produktionsfaktoren. Dies wiederum schaffte die Voraussetzungen für die Diversifizierung der landwirtschaftlichen Betriebe nach ihren sozioökonomischen Funktionen. In diesem Zusammenhang leistete die überbetriebliche Zusammenarbeit einen wesentlichen Beitrag zur Anpassung der Landwirtschaftsstruktur an die Anforderungen der sich schnell entwickelnden Volkswirtschaft. Es ist dabei allerdings anzumerken, dass allein aus der überbetrieblichen Zusammenarbeit im Bereich der Mechanisierung keine Impulse für die Einstellung der landwirtschaftlichen Tätigkeit oder eine gravierende Änderung der Betriebsgrößenstrukturen hervorgingen (vgl. ISERMEYER 1981, S. 142).

Eine erhebliche Bedeutung in der Entwicklung der Kooperation im Bereich der Maschinennutzung kam in Deutschland der staatlichen Förderung zu. Dabei handelt es sich im Wesentlichen um eine finanzielle Unterstützung für die Maschinengemeinschaften und die MR. Insbesondere im Hinblick auf die letzteren wird häufig der Sinn der jahrelangen Förderung in Frage gestellt. Befürwortet wird an dieser Stelle die ursprüngliche Anspornhilfe, die die Probleme der Anlaufphase zu überbrücken half. Kritisch wird eine Förderung beurteilt, die ein Fortdauern der MR ohne Prüfung des tatsächlichen Nutzens der Organisation für die Landwirte ermöglichte (Traulsen 2005).

Mit der Änderung der Rahmenbedingungen der Landwirtschaft ändert sich die Struktur der überbetrieblichen Maschinennutzung. Stark vereinfachend kann man von einer permanenten Bedeutungsabnahme der primären Nachbarschaftshilfe zugunsten anderer Formen der 
Zusammenarbeit sprechen. Dies kommt auch bei der Analyse der Bedeutung der MR zum Ausdruck. Die Organisation, derer Hauptfunktion in der Förderung des Maschinenleistungsaustausches zwischen benachbarten Betrieben lag, veränderte sich im Laufe der Zeit zu einem landwirtschaftsnahen gewinnorientierten Unternehmen, in dem zzt. die Vermittlung der Maschinenarbeiten generell nur eine geringe Rolle spielt. Bewährt haben sich in der deutschen Landwirtschaft, insbesondere bei mittleren Betrieben die Maschinengemeinschaften von zwei, drei Landwirten. Diese Form der Zusammenarbeit wird in vielen Betrieben als ein Instrument zur Anpassung an die sich ändernden technischen und wirtschaftlichen Rahmenbedingungen betrachtet. Eine wichtige Stelle nehmen in Deutschland auch die großen Gemeinschaften ein. Sie kommen zum Tragen insbesondere bei der Organisation ganzer Arbeitsketten mit Anwendung von kapitalaufwendigen Aggregaten.

Weiterhin vergrößert sich in der deutschen Landwirtschaft konstant auch die Bedeutung des professionell organisierten Lohnmaschineneinsatzes. Dies betrifft sowohl solche Betriebe, die auf den vorhandenen Flächen bestimmte Maschinen nicht effizient anwenden können als auch solche, in denen die Eigenmechanisierung konträr $\mathrm{zu}$ rentablen innerbetrieblichen Investitionen steht.

Einer separaten Betrachtung bedarf die Entwicklung der Mechanisierung in den östlichen Bundesländern. Aufgrund der Betriebsgrößenstrukturen sind hier die Voraussetzungen für Eigenmechanisierung wesentlich günstiger als in vielen Betrieben der westlichen Regionen. Unter diesem Gesichtspunkt kann auch die Verbreitung verschiedener Formen der überbetrieblichen Zusammenarbeit in diesen Gebieten analysiert werden.

Wenig verbreitet sind in Ostdeutschland die MR. Die MR dieser Regionen sind zwar flächenmäßig größer als die Mehrheit der MR in den westlichen Bundesländern, ihr Tätigkeitsbereich ist jedoch deutlich enger. Die Funktion der MR bei der Organisation des überbetrieblichen Maschineneinsatzes beschränkt sich hier im Wesentlichen auf die Vermittlung der Maschinenarbeit in den Zeiten der Arbeitsspitzen. Nachgefragt wird in diesen Fällen Maschinenleistung mit Bedienungspersonal. Von den Auftraggebern wird dabei das Ziel verfolgt, die Arbeitsspitzen bei relativ niedrigen Kosten der Mechanisierung und der AK $\mathrm{zu}$ brechen. Als Leistungsgeber agieren hier entweder landwirtschaftliche Betriebe mit freien Maschinenkapazitäten oder die LU. Des Weiteren ist hier, bis auf die Organisation der Betriebshilfe die Funktion der MR auch in andern Bereichen wie z.B. Rohstoffveredlung oder Handel beschränkt. Im Hinblick auf die letztere gründet sich die niedrige Aktivität der MR 
v.a. darin, dass sie nicht in der Lage sind, den großstrukturierten Betrieben über das zzt. bestehende Netz der professionellen Händler hinaus konkrete Vorteile zu bieten.

Ferner ist in diesen Regionen auch die Nachfrage nach der Dienstleistung der LU im Vergleich zu den nordwestlichen Gebieten relativ klein. Sie beschränkt sich generell auf die oben angesprochenen Arbeitsspitzen und den Einsatz spezieller Aggregate.

Ebenso wenig verbreitet ist in den östlichen Bundesländern auch der Einsatz von gemeinsamen Maschinen im Rahmen von Maschinengemeinschaften. Die gemeinsamen Maschinen werden in diesen Regionen i.d.R. in Kopperationen benutzt, die weit über den Bereich der Mechanisierung hinausgehen und primär für andere Zwecke gegründet wurden. Der Einsatz von Maschinen in kleineren Maschinengemeinschaften, der nicht ein Bestandteil von weitreichenden Zusammenschlüssen wäre, ist hier selten der Fall (Mann 2006).

Es kann festgestellt werden, dass die Gründe für die überbetriebliche Zusammenarbeit beim Maschineneinsatz zzt. weit über die ursprünglichen Ziele der Kostensenkung hinausgehen. In den Vordergrund rücken verstärkt die betriebsstrukturellen Ziele. In den westlichen Bundesländern wird die überbetriebliche Zusammenarbeit als Instrument zur Verwirklichung von Entwicklungsinvestitionen benutzt, die nur bei Interessenbindung mehrerer Betriebe durchgeführt werden können. Die Kooperation ist in vielen Fällen unausweichlich, um sich den permanent ändernden Rahmenbedingungen anzupassen oder gar die Betriebsexistenz zu sichern. Vor dem Hintergrund dieser Zielsetzung kommt die überbetriebliche Zusammenarbeit, wenn auch in wesentlich kleinerem Maße auch in den östlichen Bundesländern zum Tragen. 


\section{Der polnische Agrarsektor}

Die in den vorangegangenen Kapiteln vorgenommenen Analysen haben gezeigt, dass die natürlichen, strukturellen und organisatorischen Verhältnisse der Landwirtschaft wesentlichen Einfluss auf die Mechanisierung und die relative Vorzüglichkeit von Kooperationsformen ausüben. Daher werden hier die Verhältnisse in der polnischen Landwirtschaft als Grundlage weiterer Untersuchungen beschrieben.

\subsection{Natürliche Standortbedingungen - Klima und Boden}

Polen gliedert sich in 16 Woiwodschaften deren Gesamtfläche $312.685 \mathrm{~km}^{2}$ umfasst. Davon entfallen auf die Landfläche $304.465 \mathrm{~km}^{2}$. Vom Relief her ist Polen überwiegend als Tal- und Flachland geprägt. So liegen über $91 \%$ des Territoriums Polens unterhalb von $300 \mathrm{~m}$ über NN. Die Gebirgsgebiete befinden sich lediglich in südlichen und teilweise in landesinneren Regionen Polens. Sie gehören zu den Massiven Karpaten (mit der Tatra) und Sudeten. Die mittlere Jahresdurchschnittstemperatur in der Zeitspanne 1971- 2000 pendelt sich auf dem Niveau von $5,4^{\circ} \mathrm{C}$ im Süden (Zakopane) bis zu $8,8^{\circ} \mathrm{C}$ im Nordwesten (Szczecin) ein. Im Durchschnitt dieser Jahre beträgt die Temperatur 7,6 C. Die Jahresniederschlagsmenge liegt in den meisten Regionen des Landes bei ca. 500-700 mm/Jahr. Dabei wurde der größte Niederschlag in der Zeitspanne 1971-2000 in Zakopane (1107 mm/Jahr), der niedrigste demgegenüber in Poznan (507 L/m²/Jahr) registriert (vgl. GUS 2005).

Nach dem Bodenbewertungssystem für steuerliche Zwecke werden die Flächen in Polen in sechs Bonitätsklassen eingeteilt (siehe Übersicht 2-1, S. 7). Es werden zusätzlich für Ackerland Klassen III a und III b gebildet. Im Fall von Grünland erfolgt eine parallele Unterteilung innerhalb der Klasse IV. Während der Klasse I die Böden der besten Qualität entsprechen, werden unter der Klasse VI die schwächsten Böden subsumiert. Am stärksten sind in Polen die Böden der mittleren Qualität vertreten. So entfallen zirka 40 \% der Flächen auf die Klasse IV. Der Klasse I und II der guten und besten Böden entsprechen lediglich 3,3 \% aller Flächen.

\subsection{Landwirtschaftliche Nutzfläche und Betriebsgrößenstrukturen}

Die landwirtschaftliche Nutzfläche nahm in Polen im Jahr 2004 insgesamt 19,3 Mio. ha ein. Davon entfallen 16,9 Mio. ha auf die LF. Es herrschen in der polnischen Landwirtschaft kleine Betriebe vor. Nach der allgemeinen Definition handelt sich in Polen ab einer Flächenausstattung von 1 ha um einen landwirtschaftlichen Betrieb. Demzufolge gab es im 
Jahr 2002 fast 19,6 Mio. landwirtschaftliche Betriebe ${ }^{15}$. Ungefähr $99 \%$ dieser Gruppe machten landwirtschaftliche Einzelunternehmen des Privateigentums aus. Sie bewirtschaften auch ca. $95 \%$ aller LF des Landes. Dementsprechend entfallen auf die einigen wenigen Unternehmen des öffentlichen Sektors lediglich $5 \%$ des gesamten Areals der LF. Die durchschnittliche Flächenausstattung eines landwirtschaftlichen Betriebes beträgt ca. 8,8 ha LF. Die Unterschiede in Betriebsgrößenstruktur innerhalb des Landes werden anhand der Abbildung 4-1 verdeutlicht. Während z.B. ein landwirtschaftlicher Betrieb in Westpommern im Schnitt über 21,5 ha LF verfügt, werden in einem Betrieb in Kleinpolen oder Vorkarpaten jeweils 3,31 ha LF bzw. 3,82 ha LF bewirtschaftet.

Abbildung 4-1: Regionale Unterschiede in der Betriebsgrößenstruktur der landwirtschaftlichen Betriebe in Polen

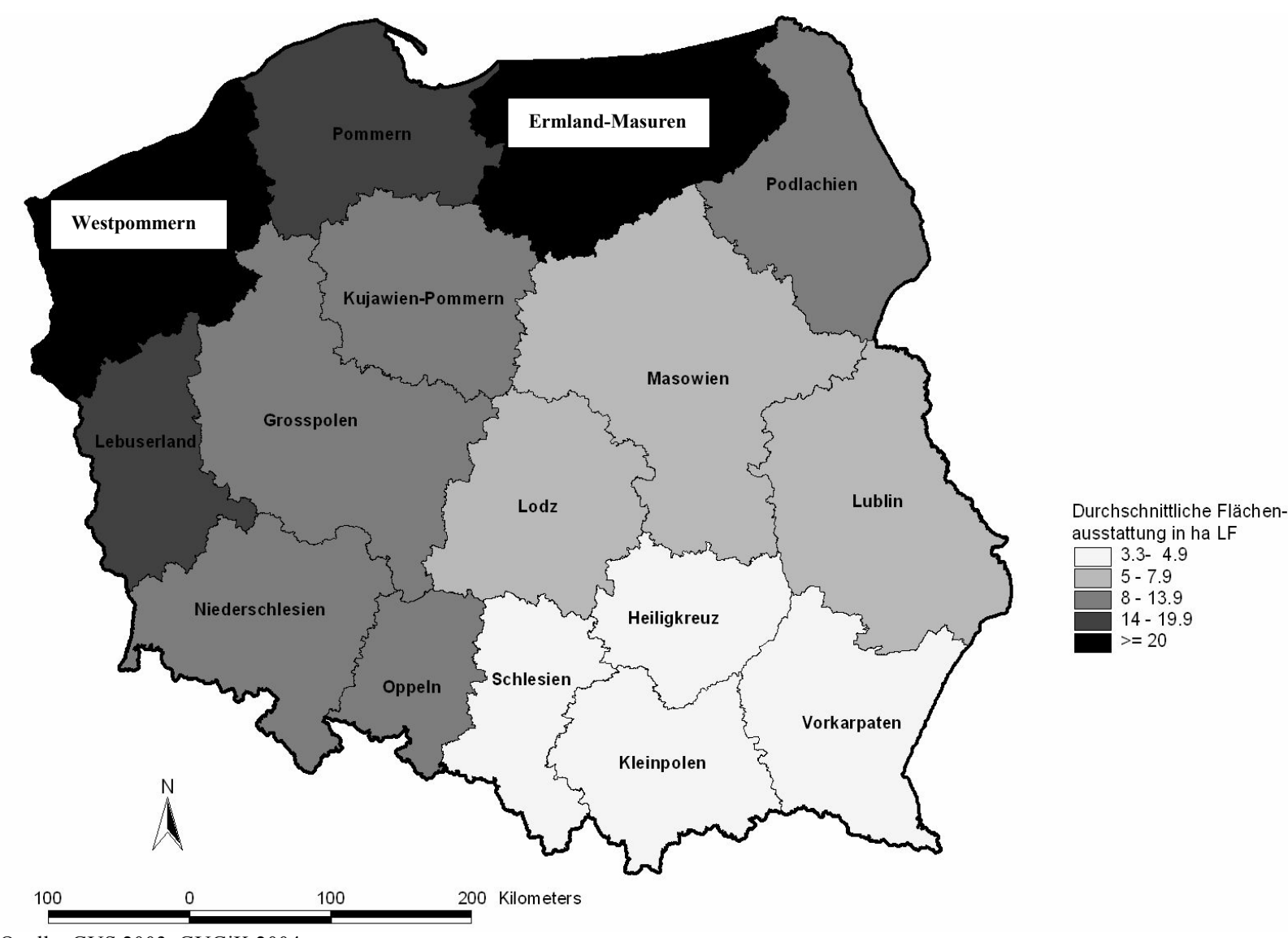

Quelle: GUS 2003, GUGiK 2004

\footnotetext{
${ }^{15}$ Die an dieser Stelle zitierten Daten stammen aus einer detaillierten flächendeckenden Landwirtschaftszählung, die in diesem Umfang zuletzt im Jahr 2002 durchgeführt wurde. Ergänzend wird hier auch auf die allgemeinen Daten aus dem Statistischen Jahrbuch 2005 zurückgegriffen. Die beiden Datenquellen bilden Grundlage aller weiteren Erörterungen dieses Kapitels.
} 
Bei einer landesweiten Betrachtung sind am stärksten vertreten die Betriebe mit einer Flächenausstattung von 1-5 ha LF. Demnach verfügen ca. $60 \%$ aller Betriebe über weniger als 5 ha LF. Die zweitgrößte Gruppe bilden Betriebe mit einer Flächenausstattung von 5-20 ha LF (35,5 \% aller Betriebe). Sehr gering ist die Anzahl der Betriebe, die mehr als 20 ha LF bewirtschaften. Lediglich 5 \% aller Betriebe Polens zählen zu dieser Gruppe.

Die Darstellung der Anteile bestimmter Größengruppen an der gesamten LF und an der Anzahl aller Betriebe verdeutlicht eine Dualität der Betriebsgrößenstruktur in der polnischen Landwirtschaft. Auf die zahlenmäßig größte Betriebsgruppe des Größenbereichs von 1-20 ha LF (ca. $94 \%$ aller Betriebe) entfallen ca. $57 \%$ aller LF. In der Bewirtschaftung einer verhältnismäßig geringen Betriebsgruppe mit einer Flächenausstattung von über 20 ha LF befinden sich ca. $47 \%$ gesamter LF. Hierzu ist auf die Abbildung 4-2 zu verweisen, welche die relativen Anteile einzelner Betriebsgroßengruppen an der gesamten LF und der Anzahl aller Betriebe darstellt.

Abbildung 4-2: Relative Anteile einzelner Betriebsgrößengruppen an der gesamten LF und der Anzahl aller Betriebe

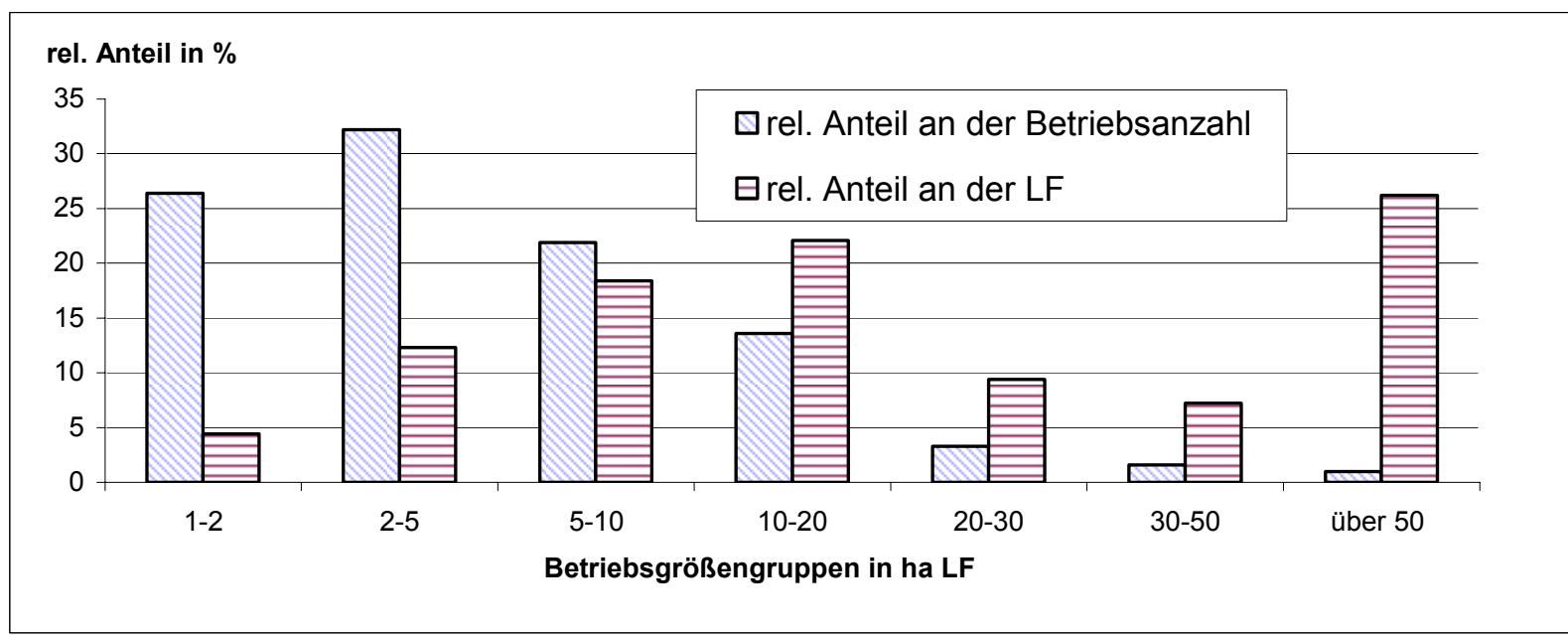

Quelle: GUS 2003

Seit dem Anfang der Transformationsprozesse zeichnen sich Änderungen in der Betriebsgrößenstruktur ab. Deutlich ging die Anzahl der mittleren Betriebe zurück. In der Zeitspanne 1996-2002 nahm die Anzahl der Betriebe mit einer Flächenausstattung von 2-20 ha LF von fast 1,5 Mio. auf 1,32 Mio. ab (siehe Tabelle 4-1). Dies entspricht einem Rückgang von über 24.500 Betriebe p.a.. Damit ging auch die Verminderung des Umfangs der in diesen Betrieben bewirtschafteten LF einher. Während auf die Betriebe dieser Größengruppe im Jahr 1996 noch 59,5 \% aller LF entfielen, schrumpfte dieser Anteil bis zum Jahr 2002 auf 52,8\%. Weiterhin ist in der angesprochenen Zeitspanne eine Zunahme der Betriebe der Größengruppe von über 20 ha LF zu verzeichnen. Die Anzahl der Betriebe dieser 
Größengruppe nahm um ca. $12 \%$ zu, zugleich vergrößerte sich das Volumen der in diesen Betrieben bewirtschafteten LF um ca. $13 \%$.

Tabelle 4-1: Struktur der landwirtschaftlichen Betriebe in den Jahren 1995- 2002

\begin{tabular}{|c|c|c|c|c|c|c|c|c|}
\hline \multirow{2}{*}{$\begin{array}{c}\text { Betriebsgröße } \\
\text { in ha LF }\end{array}$} & \multicolumn{9}{|c|}{1996} & \multicolumn{4}{|c|}{2002} \\
\cline { 2 - 9 } & $\begin{array}{c}\text { Zahl } \\
\text { in } 1000\end{array}$ & $\%$ & $\begin{array}{c}\text { Fläche } \\
\text { in } 1000\end{array}$ & $\%$ & $\begin{array}{c}\text { Zahl } \\
\text { in } 1000\end{array}$ & $\%$ & $\begin{array}{c}\text { Fläche } \\
\text { in } 1000\end{array}$ & $\%$ \\
\hline $1-2$ & 462,4 & 22,6 & 650,9 & 3,8 & 517 & 26,4 & 725,3 & 4,4 \\
$2-5$ & 667,9 & 32,6 & 2200,3 & 13 & 629,9 & 32,2 & 2039,2 & 12,3 \\
$5-10$ & 521,1 & 25,5 & 3715,7 & 21,9 & 426,9 & 21,9 & 3031,6 & 18,4 \\
$10-20$ & 306,9 & 15 & 4166,2 & 24,6 & 266,6 & 13,6 & 3656,3 & 22,1 \\
$20-30$ & 55,8 & 2,7 & 1327,3 & 7,8 & 64,3 & 3,3 & 1541,2 & 9,4 \\
$30-50$ & 19,8 & 1 & 729,7 & 4,3 & 31,7 & 1,6 & 1181,5 & 7,2 \\
50 und mehr & 12,6 & 0,6 & 4178,6 & 24,6 & 19,8 & 1 & 4327,7 & 26,2 \\
\hline gesamt & 2046,5 & 100 & 16968,7 & 100 & 1956,2 & 100 & 16502,8 & 100 \\
\hline
\end{tabular}

Des Weiteren deuten die statistischen Daten auf eine starke Zunahme der Anzahl der kleinsten Betriebe hin. Die Anzahl der Betriebe mit einer Flächenausstattung von 1-2 ha LF nahm um insgesamt $12 \%$ zu. Die Ursache hierfür liegt zumindest teilweise darin, dass mit dem Bodeneigentum, somit dem Status Landwirt, in Polen bestimmte Vorteile verbunden sind. Es handelt sich hier v.a. um den Genuss besonderer Vergünstigungen bei der landwirtschaftlichen Sozialversicherung. Hinzu kommt, dass der Bodenerwerb, insbesondere in den letzten Jahren als eine günstige Vermögensanlage betrachtet wurde. Eine besondere Bedeutung hatte an dieser Stelle der Erwerb relativ kleiner landwirtschaftlicher Flächen, die wegen ihrer Lage eine außerlandwirtschaftliche Nutzung und eine damit verbundene Werterhöhung erwarten lassen.

\subsection{Bodennutzung}

Von den insgesamt 16,9 Mio. ha LF entfallen ca. $77 \%$ auf das Ackerland und ca. $21 \%$ auf das Dauergrünland. Nahezu $2 \%$ aller LF werden der Kategorie Gartenland zugeordnet. Innerhalb des Ackerlandes wird zwischen bebauten und brachgelegten Flächen unterschieden. Dementsprechend wurden im Jahr 2002 ca. 18 \% des Ackerlandes nicht bewirtschaftet. Die Anteile verschiedener Fruchtarten an den bebauten Ackerflächen werden in der Abbildung 43 dargestellt. Die meisten AF werden für den Getreideanbau beansprucht. Dabei handelt es sich v.a. um Weizen- und Roggenanbau, auf den jeweils $37 \%$ bzw. $24 \%$ der Getreideanbauflächen entfallen. 
Abbildung 4-3: Relative Anteile der Anbauflächen an den Ackerflächen im Jahr 2002

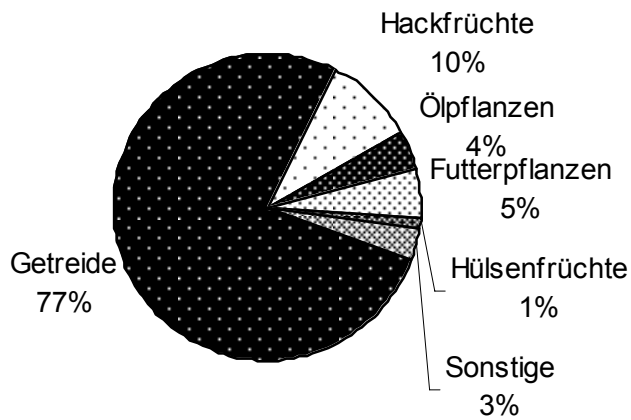

Quelle: GUS 2003

Die Anteile bestimmter Nutzungsarten an der gesamten LF sind von Region zu Region unterschiedlich. Wie die Abbildung 4-4 zeigt, ist der Anteil von bebautem Ackerland am höchsten in Woiwodschaften Oppeln und Großpolen, dabei handelt es sich um den südlichen und mittleren Teil des Landes. Die größten Anteile von Brachflächen sind für die südöstlichen Regionen kennzeichnend. Es handelt sich dabei zum großen Teil um marginale Standorte, auf denen kaum eine rentable Pflanzenproduktion durchzuführen ist. Es ist dabei allerdings anzumerken, dass die meisten Brachflächen des Landes in den nördlichen Regionen vorhanden sind. Dies ist wiederum darauf zurückzuführen, dass in diesen Gebieten viele Flächen aus den ehemaligen LPG immer noch nicht vollständig privatisiert worden sind. Die höchsten Anteile von Grünland zeichnen die nordöstlichen Regionen aus. In drei Woiwodschaften Ermland - Masuren, Podlachien und Masowien befinden sich fast $40 \%$ des gesamten Dauergrünlandes Polens. Dem Obstanbau kommt eine besondere Bedeutung in dem mittleren und östlichen Teil des Landes zu. Hier ist sowohl die Anzahl der Betriebe mit Obstanbau als auch die gesamte Fläche der Obstanlagen mit deutlichem Abstand am größten. 
Abbildung 4-4: Relative Anteile der Flächennutzungsarten nach Woiwodschaften

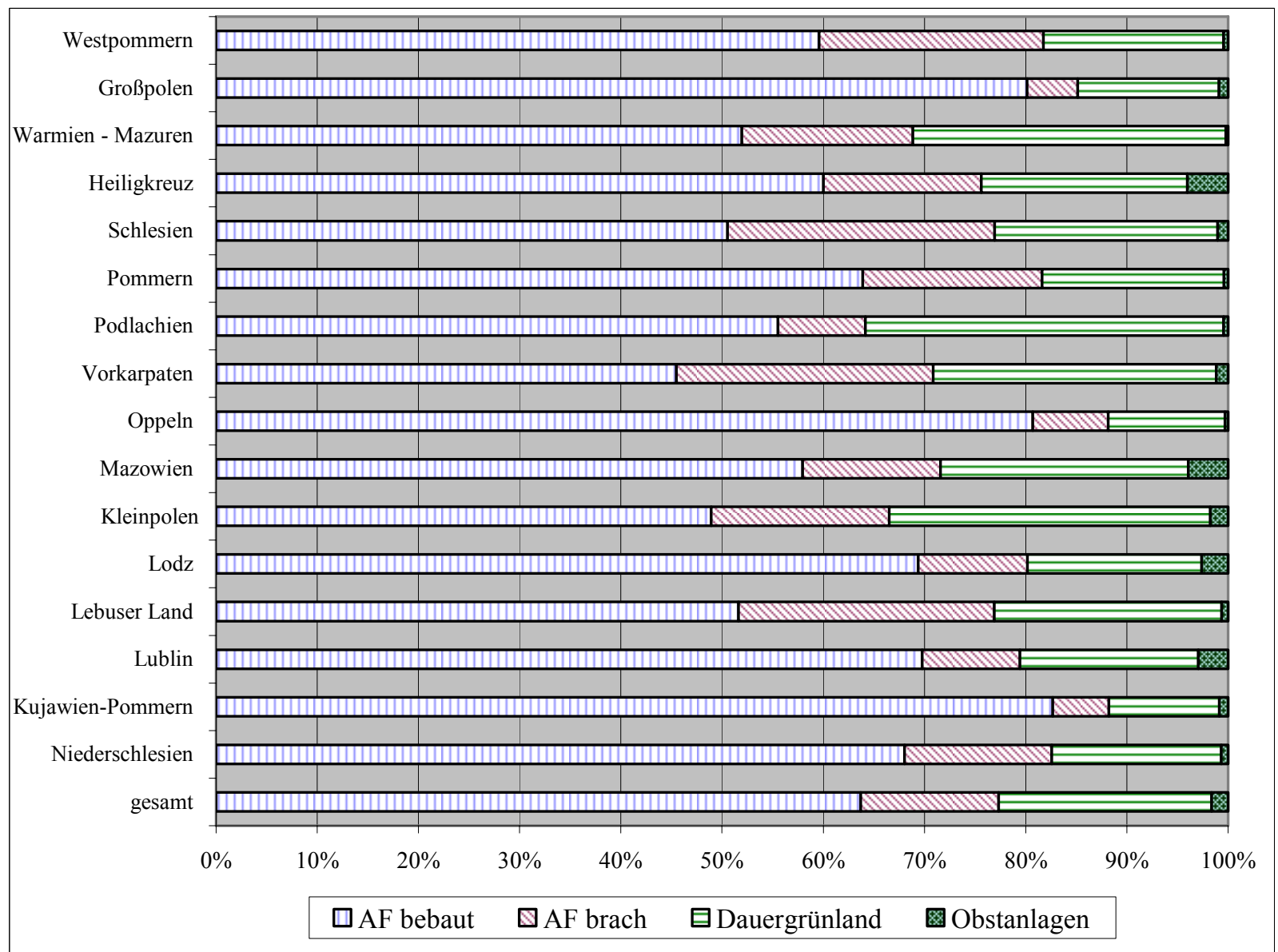

Quelle: GUS 2003

\subsection{Produktionsstruktur}

Das Gesamtbild der polnischen Landwirtschaft ist stark von Gemischtbetrieben geprägt, in denen die Produktion unter Bedingungen starker Zersplitterung erfolgt. Die Anzahl von Betrieben mit einem oder nur wenigen Betriebszweigen ist niedrig. Auf einem unveränderlich hohen Niveau beläuft sich der Anteil von Subsistenzwirtschaften. In ungefähr 1,7 \% aller im Jahr 2002 statistisch erfassten landwirtschaftlichen Betriebe wurde keine landwirtschaftliche Produktion betrieben. Von den verbliebenen Betrieben produzieren:

$-\quad 20,4 \%$

- $\quad 36,3 \%$

- $\quad 43,3 \%$ ausschließlich für den eigenen Bedarf, vorwiegend für den eigenen Bedarf und verkauften gelegentliche Überschüsse, vorwiegend und regelmäßig für den Markt. 
Weiterhin erfolgt die Tierhaltung in den landwirtschaftlichen Betrieben Polens zum großen Teil in kleinen und sehr kleinen Beständen. In ca. $48 \%$ der Rinderhaltungsbetriebe werden bis zu 2 Rinder gehalten. Der Anteil der Betriebe mit Beständen von mehr als 20 Tieren geht nicht über $5,3 \%$ aller Tierhaltungsbetriebe hinaus. Ein ähnliches Bild stellt die Produktionsstruktur in den Schweinehaltungsbetrieben dar. In ungefähr $24 \%$ aller Betriebe werden im Schnitt weniger als zwei Schweine gehalten. Der Anteil der Betriebe mit mehr als 20 Tieren liegt bei ca. $28 \%$. Die Entwicklung der Struktur der Tierhaltungsbetriebe in den Jahren 1996-2003 wird numerisch in der Tabelle 4-2 dargestellt.

Tabelle 4-2: Struktur der Rinder- und Schweinebestände in den landwirtschaftlichen Betrieben in den Jahren 1996-2002

\begin{tabular}{|c|c|c|c|c|}
\hline \multirow{2}{*}{$\begin{array}{c}\text { Tierbestand } \\
\text { in Stück }\end{array}$} & \multicolumn{4}{|c|}{ Anteil der Betriebe in \% } \\
\cline { 2 - 5 } & 1996 & 2002 & 1996 & 2002 \\
\cline { 2 - 5 } & 44,1 & 48,3 & 28,9 & 24 \\
$3-9$ & 44,3 & 36,1 & 34,1 & 28,8 \\
$10-19$ & 9,3 & 10,2 & 18,5 & 28,2 \\
20 und mehr & 2,3 & 5,4 & 18,5 & 28 \\
\hline
\end{tabular}

Die Verteilung der Tierbestände in bestimmten Betriebsgrößengruppen veranschaulicht, dass die Betriebe mit einer Flächenausstattung von 7-30 ha LF die größten Anteile an der Tierproduktion haben (siehe Abbildung 4-5). Verhältnismäßig hoch ist die Bedeutung der Tierhaltung auch in den Betrieben des Größenbereichs von 200 und mehr ha LF. Die geringen Anteile der Tierbestände in den Betrieben der Größengruppe 1-7 ha LF bestätigen die These über die Subsistenzwirtschaften, in denen die Tierhaltung im Wesentlichen der Deckung des eigenen Nahrungsmittelbedarfs dient. An dieser Stelle ist anzumerken, dass die Angaben dieser Abbildung nicht den aktuellen Stand der Tierhaltung exakt wiederzugeben vermögen. Wegen der andauernden Änderungen der Tierbestände sind die Angaben sehr zeitpunktbezogen $\mathrm{zu}$ betrachten. Daher können sie lediglich als ein Indiz der Produktionsstruktur der landwirtschaftlichen Betriebe Polens dienen. 
Abbildung 4-5: Verteilung der Rinder- und Schweinebestände auf die einzelnen Betriebsgrößengruppen

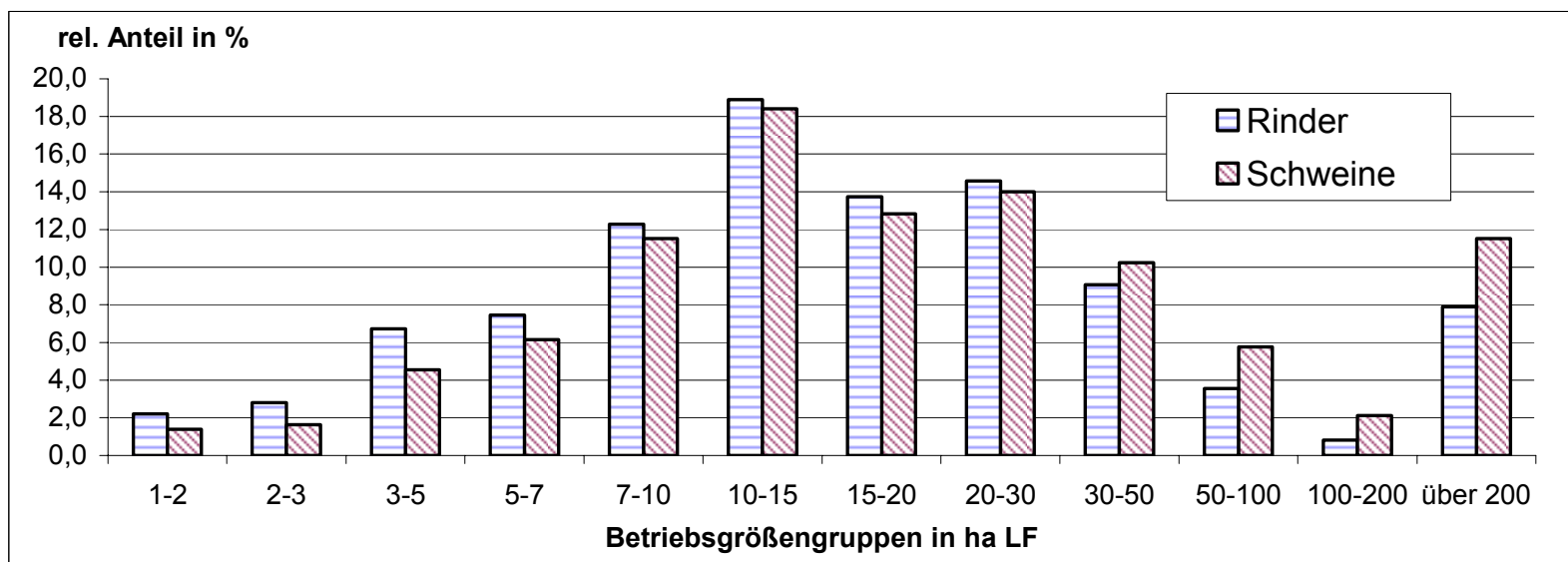

Quelle: GUS 2003

\subsection{Eigentums- und Pachtverhältnisse}

Die in den Zeiten der sozialistischen Regierung verfolgte Idee der Verstaatlichung des privaten Eigentums wurde im Fall der polnischen Landwirtschaft, im Vergleich zu anderen Ländern des sozialistischen Blocks, nur in einem beschränkten Maße verwirklicht. Der Anteil der sich im Staatseigentum befindlichen LF ging nie über $25 \%$ hinaus (vgl. GUS, DIVERSE JAHRGÄNGE). Wenngleich das Privateigentum nicht völlig zerstört wurde, leistete die Politik der Verstaatlichung einen wesentlichen Beitrag zur Stagnation und einer andauernden Dominanz der flächenmäßig kleinen Betriebe.

Derzeit werden in den landwirtschaftlichen Betrieben des privaten Sektors insgesamt 94,5\% aller LF des Landes bewirtschaftet. Der Anteil der Familienbetriebe an allen Betrieben des Privatsektors beläuft sich auf ca. 88 \%. Wie bereits angesprochen, handelt es sich dabei zum großen Teil um kleine bzw. sehr kleine Betriebe. Eine ungünstige Wirtschaftslage und ein anhaltender Mangel an Arbeitsstellen außerhalb der landwirtschaftlichen Betriebe geben keinen Ansporn für die Abwanderung der AK aus der Landwirtschaft. Die LF werden aufgrund ihrer seit Jahren konstanten Werterhöhung als langfristige Kapitalanlage betrachtet und generell ungern verkauft. Dies hat zur Folge, dass die Eigentumsumschichtungen im Bereich der LF in einem eher langsamen Tempo erfolgen. Wie die Abbildung 4-6 zeigt, hat in der Änderung der Besitzverhältnisse eine deutlich größere Bedeutung die Flächenpacht als der Flächenkauf. Der Umfang der gepachteten LF nahm zwischen 1991-1999 um ca. 93 \% zu. 
Abbildung 4-6: Kauf und Pacht der landwirtschaftlichen Flächen in den Jahren 1991-2005 ${ }^{16}$

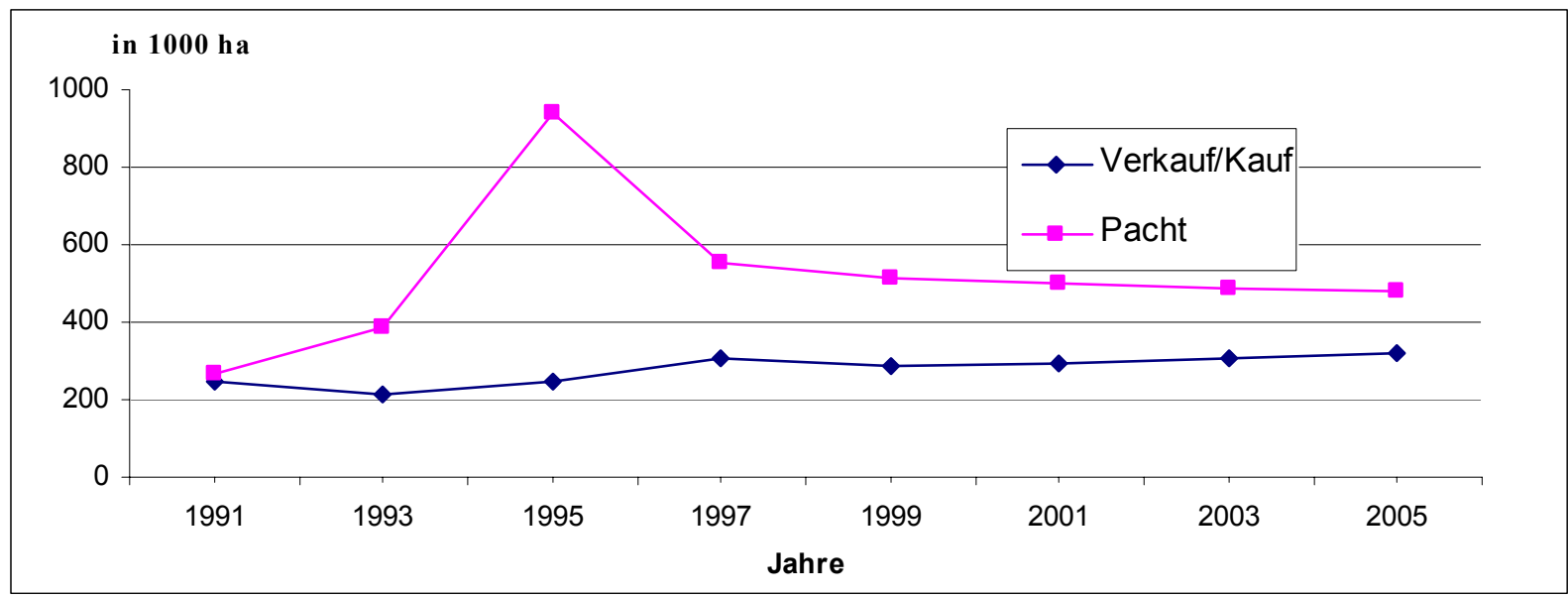

Quelle: Ostrowski 2000, S.8; ANR 2005

Trotz der zunehmenden Bedeutung der Flächenpacht ist der Anteil der Betriebe mit Pachtflächen nach wie vor niedrig. Demzufolge machten im Jahr 2003 die Betriebe mit Pachtflächen ca. $14 \%$ aller Betriebe aus. Der Anteil der Betriebe, die ausschließlich Pachtflächen bewirtschafteten, lag im demselben Jahr bei ca. $2 \%$ (vgl. SIKORSKA et. all 2004, S. 17).

Die durchschnittliche Größe der Pachtflächen ist von Region zu Region unterschiedlich. Während im Norden und Nordwesten überwiegend größere Flächen von 10 ha und mehr gepachtet werden, sind die Pachtflächen in südöstlichen Regionen generell nicht größer als 5 ha. Die durchschnittliche Größe der Pachtflächen im zwischenbetrieblichen Verkehr beläuft sich auf ungefähr 3 ha. Die Pacht zwischen einzelnen landwirtschaftlichen Betrieben erfolgt i.d.R. informell anhand von mündlichen Vereinbarungen. Zum Pachtgeschehen im privaten Verkehr liegen aktuell bis auf die genannten Anteile der Betriebe mit Pachtflächen und die durchschnittliche Flächengröße, keine einheitlichen Daten vor.

Eine wesentliche Bedeutung am Bodenmarkt haben die Flächen aus dem landwirtschaftlichen Staatseigentum (WRSP), die zzt. durch die Agentur für landwirtschaftliche Immobilien (ANR) verwaltet werden ${ }^{17}$. Das Instrument der Flächenpacht erlangte im Laufe der Umstrukturierung des Staateigentums größere Bedeutung als der Verkauf. Bis zum Jahr 2004

\footnotetext{
${ }^{16}$ Einschließlich Flächen von WRSP.

${ }^{17}$ Für die Übernahme und anschließende Privatisierung der im Staatseigentum befindlichen Liegenschaften aus dem ehemaligen Bodenfond und den staatlichen Landunternehmen wurde im Jahr 1992 die AWRSP (Agentur für landwirtschaftliches Eigentum des Staatseigentums) ins Leben gerufen (vgl. Dz.U. Nr.1991.107.464; Dz.U. Nr.1992.24.104 mit späteren Änderungen). Die AWRSP wurde gemäß dem Agrargesetz vom 11.04.03 (Dz.U.Nr.03.64.592) unbenannt und trägt zzt. den Namen Agentur für landwirtschaftliche Immobilien (ANR).
} 
wurde lediglich ein Drittel der übernommenen Flächenressourcen des landwirtschaftlichen Staatseigentums auf dem Weg des Verkaufs verwaltet (SzCZUROWNA 2004).

Ein Aufschwung in der Verpachtung der Flächen aus WRSP ist für die Jahre 1994-1995 signifikant. Wegen des in den Folgejahren erfolgten Verkaufs oder der langfristigen Verpachtung der günstigsten Flächen, nimmt die Anzahl der abgeschlossenen Pachtverträge und somit das Pachtflächenvolumen deutlich ab. In der Mehrheit der zzt. vorhandenen Verträge handelt es sich um Liegenschaften von 1 ha bis zu 10 ha. Die weiteren $10 \%$ der Verträge umfassen Liegenschaften von 10 ha bis 100 ha. Lediglich 2,3\% aller Verträge betreffen Liegenschaften der Größenordnung von 100 ha und mehr (siehe Tabelle 4-3).

Tabelle 4-3: Pachtflächen von WRSP

\begin{tabular}{|c|c|c|c|c|c|c|}
\hline Jahre & 1994 & 1996 & 1998 & 2000 & 2002 & 2004 \\
\hline Fläche in 1000 ha & 1981 & 2928 & 2810 & 2618 & 2508 & 2349 \\
\hline $\begin{array}{c}\text { Fläche in \% des } \\
\text { ges. Flächenvolumens }\end{array}$ & 47 & 65 & 60 & 56 & 51 & 50 \\
\hline \multicolumn{7}{|c|}{ Größenstruktur der Pachtflächen am 31.12.03 } \\
\hline \multirow[t]{2}{*}{ Flächengröße } & \multicolumn{2}{|c|}{ Zahl der Pachtverträge } & \multicolumn{2}{|c|}{ verpachtete Fläche } & \multirow{2}{*}{\multicolumn{2}{|c|}{ Fläche pro Vertrag }} \\
\hline & insgesamt & in $\%$ & in 1000 ha & in $\%$ & & \\
\hline $0,1-10$ ha & 130078 & 87,5 & 220,4 & 10,4 & & \\
\hline $10-100$ ha & 15019 & 10 & 427,9 & 20 & & \\
\hline $100-500$ ha & 2696 & 2 & 671,6 & 31,5 & & \\
\hline 500 und mehr & 779 & 0,5 & 811,9 & 38,1 & & \\
\hline
\end{tabular}

Quelle: Szczurowna 2004, ANR 2004

Der Pachtpreis für Flächen der ANR bildet das Äquivalent von Weizen, das je nach der Region und Struktur der Pachtflächen ein unterschiedliches Niveau erreicht (siehe Tabelle 4-4).

Tabelle 4-4: Durchschnittliche Boden- und Pachtpreise im Jahr 2004

\begin{tabular}{|l|c|c|c|}
\hline \multirow{2}{*}{ Woiwodschaften } & \multicolumn{2}{|c|}{ Bodenpreise pro 1 ha } \\
\cline { 2 - 4 } & privat & \multicolumn{2}{|c|}{ ANR } \\
\cline { 2 - 4 } Kleinpolen & Kauf in ZL & Kauf in ZL & Pachtentgelt in dt /Weizen \\
Mazowien & 7874 & 6829 & 2,8 \\
Lublin & 8039 & 4886 & 8,5 \\
Vorkarpaten & 5165 & 3493 & 2,0 \\
Ermland- Masuren & 5285 & 3061 & 1,7 \\
Westpommern & 5232 & 3989 & 2,8 \\
\hline
\end{tabular}

\footnotetext{
${ }^{18}$ Umrechnungskurs: 1 ZL=0,26 EUR.
} 


\subsection{AK- Ausstattung}

Die Analyse der Zahlen zur Beschäftigungsstruktur zeigt ein wesentliches Defizit in der Arbeitsproduktivität in der Landwirtschaft auf. Im Jahr 2004 waren in den landwirtschaftlichen Betrieben ca. 1,96 Mio. AK beschäftigt. Es handelt sich hier überwiegend um Familienarbeitskräfte. Der Anteil von familienfremden ständig beschäftigten AK an der gesamten Anzahl aller in der Landwirtschaft beschäftigten Personen liegt bei ca. $2 \%$. Der Arbeitsaufwand in JAE der in landwirtschaftlichen Betrieben tätigen Familienmitglieder wird anhand der Tabelle 4-5 zum Ausdruck gebracht. Der bereits geschilderten Betriebsgrößenstruktur entsprechend, werden die meisten $\mathrm{AK}$ in den Betrieben der Größengruppen 1-15 ha LF gebunden. Auf die Betriebe mit einer Flächenausstattung von mehr als 15 ha LF entfällt weniger als ein Fünftel aller AK.

Tabelle 4-5: Arbeitsaufwand in $1000 \mathrm{JAE}$ in landwirtschaftlichen Betrieben bestimmter Größengruppen

\begin{tabular}{|c|c|c|c|c|c|c|c|c|c|c|c|}
\hline $\begin{array}{r}\text { Betriebsgröße } \\
\text { in ha LF }\end{array}$ & $1-2$ & $2-5$ & $5-7$ & $7-10$ & $10-15$ & $15-20$ & $20-30$ & $30-50$ & $\begin{array}{c}50- \\
100\end{array}$ & $\begin{array}{c}\text { über } \\
100\end{array}$ & gesamt \\
AK nach Status & 127,7 & 289,8 & 143,8 & 160,7 & 154,2 & 74,7 & 58,5 & 28,7 & 10,5 & 3,9 & 1052,5 \\
\hline $\begin{array}{c}\text { Betriebsleiter } \\
\begin{array}{c}\text { Ehegatten des } \\
\text { Betriebsleiters }\end{array}\end{array}$ & 56,9 & 136,1 & 72,3 & 85,9 & 88,9 & 46,2 & 38,1 & 19,1 & 6,7 & 2,1 & 552,3 \\
\hline $\begin{array}{c}\text { andere } \\
\text { Familienangehörige }\end{array}$ & 30,7 & 95,9 & 50,3 & 55,6 & 55,5 & 27,9 & 23,1 & 12,2 & 4,6 & 1,5 & 357,3 \\
\hline $\begin{array}{c}\text { insgesamt } \\
\text { in \% }\end{array}$ & 215,3 & 521,8 & 266,4 & 302,2 & 298,6 & 148,8 & 119,7 & 60 & 21,8 & 7,5 & 1962,1 \\
\hline Quelle: GUS 2003 & 11,0 & 26,6 & 13,6 & 15,4 & 15,2 & 7,6 & 6,1 & 3,1 & 1 & 0,4 & 100 \\
\hline
\end{tabular}

In der Zeitspanne 1996-2002 sind wesentliche Unterschiede in der Struktur der Unterhaltungsquellen der ländlichen Bevölkerung zu verzeichnen. Wesentlich verkleinert sich die Gruppe der Personen, die ihren Lebensunterhalt ausschließlich durch das Einkommen aus der Arbeit im eigenen landwirtschaftlichen Betrieb bestreiten. Diese Entwicklung wird allerdings nur in einem geringen Maße durch die Aufnahme einer außerlandwirtschaftlichen Beschäftigung verursacht. Weiter steigt stark die Anzahl der Personen an, die von anderen Familienangehörigen finanziell unterhalten werden. Ungefähr ein Viertel aller mit den landwirtschaftlichen Betrieben verbunden Personen unterhält sich vom Einkommen aus Nichterwerbsquellen, dabei handelt es sich hauptsächlich um Personen, die ArbeitsErwerbsunfähigkeitsrenten erhalten (siehe Tabelle 4-6). 
Tabelle 4-6: Status der mit den landwirtschaftlichen Betrieben verbundenen Personen nach Art der Unterhaltsquelle in den Jahren 1996-2002 in \%

\begin{tabular}{|l|c|c|c|}
\hline & 1996 & 2002 & 2002 gegen 1996 \\
\hline $\begin{array}{l}\text { Beschäftigung ausschließlich } \\
\text { im landwirtschaftlichen Betrieb }\end{array}$ & 16 & 12,3 & $+3,7$ \\
\hline $\begin{array}{l}\text { Beschäftigung hauptsächlich } \\
\text { im landwirtschaftlichen Betrieb }\end{array}$ & 1,6 & 1,5 & $-0,1$ \\
\hline $\begin{array}{l}\text { Beschäftigung ausschließlich } \\
\text { oder hautsächlich außerhalb von land. Betrieb }\end{array}$ & 20,6 & 21,2 & $+0,6$ \\
\hline $\begin{array}{l}\text { Einkommen aus einer ausschließlich oder } \\
\text { hauptsächlich nicht erwerbsmäßigen Quelle }\end{array}$ & 28,5 & 25,8 & $-2,7$ \\
\hline Unterhalt als Familienmitglied & 32,8 & 38,8 & +6 \\
\hline Ohne Angabe der Unterhaltsquelle & 0,5 & 0,4 & -01 \\
\hline
\end{tabular}

Quelle: GUS 2003

\subsection{Arbeitsmarkt}

Die Entwicklung der Beschäftigungsstruktur in der polnischen Landwirtschaft ist eng mit den gesamten wirtschaftspolitischen Prozessen der letzten Jahre verbunden. In den sozialistischen Zeiten bildete die ländliche Bevölkerung das Arbeitskraftpotenzial für Industrie und staatliche Landwirtschaftsunternehmen. Die im Zuge der Restrukturierung der polnischen Wirtschaft registrierte erhebliche Zunahme der Arbeitslosigkeit betraf zum großen Teil die ländliche Bevölkerung. Unter diesen Umständen erlangte die Arbeit im landwirtschaftlichen Betrieb eine wesentliche Bedeutung als eine Art Beschäftigungsalternative.

Die Arbeitslosenquote in Polen nahm als Effekt der Restrukturierung von 1990 bis 1994 kontinuierlich von $6,1 \%$ auf $16 \%$ zu (siehe Tabelle 4-7). Die danach einsetzende Konjunkturbelebung führte zu einer Beschäftigungszunahme und $\mathrm{zu}$ einer Minderung der Arbeitslosenquote bis zum Jahr 1998 auf 10,4 \%. Im Jahresdurchschnitt 2000 war die Anzahl der registrierten Arbeitslosen bereits wieder auf 2,7 Mio. angestiegen (Arbeitslosenquote von 15,1\%). Seitdem nahm die Arbeitslosenquote bis zum Jahr 2002 wieder auf 20 \% zu. Im Jahr 2005 wurden fast 3 Mio. Arbeitslose registriert. Davon entfallen ca. $35 \%$ auf die ländliche Bevölkerung. Neben den registrierten Arbeitslosen existiert eine beachtliche stille Reserve an Arbeitslosen, die in den statistischen Daten nicht aufgedeckt wird. 
Tabelle 4-7: Entwicklung der Anzahl der Erwerbstätigen in den Jahren 1990-2004

\begin{tabular}{|c|c|c|c|c|}
\hline Jahr & $\begin{array}{c}\text { Anzahl aller } \\
\text { Erwerbstätigen } \\
\text { in 1 } 000\end{array}$ & $\begin{array}{c}\text { Erwerbstätige in der } \\
\text { Land- und } \\
\text { Forstwirtschaft } \\
\text { in } 1000\end{array}$ & $\begin{array}{c}\text { Anteil der } \\
\text { Erwerbstätigen in der } \\
\text { Land- und } \\
\text { Forstwirtschaft }\end{array}$ & $\begin{array}{c}\text { Arbeitslosenquote in } \\
\%\end{array}$ \\
\hline 1990 & 16474 & 4844 & 29,4 & 6,1 \\
\hline 1992 & 15010 & 4475,1 & 29,8 & 13,6 \\
\hline 1994 & 14924 & 4039,5 & 27,1 & 13,2 \\
\hline 1996 & 15487 & 4358,7 & 28,1 & 10,4 \\
\hline 1998 & 15921 & 4346,5 & 27,3 & 15,1 \\
\hline 2000 & 15489 & 4304,6 & 27,7 & 20 \\
\hline $2002^{19}$ & 12641 & 2138,3 & 16,9 & 19,1 \\
\hline 2004 & 12737 & 2144,2 & 16,8 & \\
\hline
\end{tabular}

Bei einem regionalen Vergleich innerhalb Polens sind wesentliche Disproportionen auf dem Arbeitsmarkt erkennbar. Die Arbeitslosigkeit, bedingt durch niedrige Entwicklung anderer Wirtschaftszweige, ist mit deutlichem Abstand am höchsten in den nördlichen Regionen des Landes, wo die Wirtschaft zu sozialistischen Zeiten im Wesentlichen durch die staatlichen Landunternehmen geprägt wurde. In Regionen mit gegenteiligen Bedingungen ist die Arbeitslosigkeit, insbesondere in der Nähe größerer Ballungszentren, vergleichsweise niedrig. Während im mittleren Teil des Landes die Arbeitslosenquote generell nicht über $15 \%$ hinausgeht, reicht sie im nördlichen Teil Polens bis zu 30 \%.

\subsection{Mechanisierungsstruktur}

Die Mehrheit der Maschinenarbeiten wird in den landwirtschaftlichen Betrieben in Polen in der Eigenmechanisierung organisiert. Der überbetriebliche Maschineneinsatz kam bisher generell nur in der Form der Nachbarschaftshilfe sowie der Dienstleistung der sog. Genossenschaften der landwirtschaftlichen Zirkel zutage. In junger Vergangenheit wurde versucht, die Idee der überbetrieblichen Maschinennutzung zu popularisieren. Hierzu zählt die Gründung einiger MR nach dem deutschen Vorbild und die im Jahr 1997 in Form von zinsverbilligten Krediten aktivierte Förderung für eine gemeinsame Anschaffung von bestimmten Maschinen und Geräten im sog. Branchenprogramm BR/10 (siehe hierzu auch S. 90 f).

Im Jahr 2002 gab es in der polnischen Landwirtschaft über 1,3 Mio. Schlepper. Demzufolge entfallen auf 100 ha LF etwa 8 Schlepper. Dabei bilden die zahlreichste Gruppe die Schlepper

\footnotetext{
${ }^{19}$ Infolge der Änderungen der Erfassungsmethodik der Erwerbstätigen in dem landwirtschaftlichen Verzeichnis 2002 sind die Angaben für die Jahre 2002 und 2004 mit denen der Vorjahre nicht vergleichbar. Ein wesentlicher Unterschied in der Erfassung der Erwerbstätigen betrifft die Anzahl der in der Landwirtschaft tätigen Personen. Seit dem Jahr 2002 zählen zu dieser Gruppe nicht die Personen aus den landwirtschaftlichen Betrieben, in denen die landwirtschaftliche Produktion ausschließlich für den eigenen Bedarf erfolgt.
} 
mit einer Motorleistung von 28-29 KW (ca. $49 \%$ aller Schlepper). Auf die zweitgrößte Schleppergruppe mit einer Motorleistung von 29-44 kW entfallen ca. 36,6 \% aller Schlepper. Lediglich 2,4 \% aller in den landwirtschaftlichen Betrieben vorhandenen Schlepper weist eine Motorleistung von über $80 \mathrm{~kW}$ auf (siehe Tabelle 4-8).

Die Mechanisierung der landwirtschaftlichen Betriebe nimmt, gemessen an der Anzahl und der Motorleistung der Schlepper in kW pro 100 ha LF konstant zu. Entfielen im Jahr 1996 auf 100 ha LF ca. 227 kW, so stand dieser Flächeneinheit im Jahr 2003 eine Motorleistung von 278 kW gegenüber. Das einem Schlepper entsprechende Flächenvolumen verringert sich von 14 ha auf 12,4 ha LF. Erkennbar sind wesentliche Unterschiede in dem Mechanisierungszustand bestimmter Betriebsgrößengruppen. Während in den Betrieben des Größenbereichs von 1-50 ha LF pro 100 ha des Ackerlandes durchschnittlich $400 \mathrm{~kW}$ entfallen, stehen 100 ha des Ackerlandes in den Betrieben mit 50 und mehr ha LF durchschnittlich $110 \mathrm{~kW}$ Motorleistung gegenüber (Muzalewski 2005).

Tabelle 4-8: Zahl der Schlepper nach Motorleistung und Betriebsgröße

\begin{tabular}{|c|c|c|c|c|c|c|c|c|c|}
\hline & \multicolumn{6}{|c|}{ Motorleistung in $\mathrm{kW}(\mathrm{PS})$} \\
\hline & & & & $\begin{array}{c}\text { bis } 18 \\
(24)\end{array}$ & $\begin{array}{c}18-29 \\
(24-39)\end{array}$ & $\begin{array}{c}29-44 \\
(39-60)\end{array}$ & $\begin{array}{c}44-81 \\
(60-110)\end{array}$ & $\begin{array}{c}\text { über } 81 \\
\text { (über 110) }\end{array}$ & Insgesamt \\
\hline \multicolumn{4}{|c|}{$\begin{array}{l}1996 \\
\text { Zahl der Schlepper }\end{array}$} & 40.600 & 668.572 & 479.434 & 84.332 & 24.934 & 1297.872 \\
\hline \multicolumn{4}{|c|}{ Motorleistung in $\mathrm{kW}$} & $\begin{array}{c}354 \\
(481)\end{array}$ & $\begin{array}{c}15725 \\
(21386)\end{array}$ & $\begin{array}{c}16914 \\
(23003)\end{array}$ & $\begin{array}{c}4996 \\
(6795)\end{array}$ & $\begin{array}{c}2492 \\
(3389)\end{array}$ & $\begin{array}{c}40481 \\
(55054)\end{array}$ \\
\hline \multicolumn{4}{|c|}{ Motorleistung in $\mathrm{kW}$ (PS) je 100 ha LF } & \multicolumn{6}{|c|}{$227(306)$} \\
\hline \multicolumn{4}{|l|}{$\begin{array}{l}2003 \\
\text { Zahl der }\end{array}$} & 40.934 & 680.149 & 505.759 & 119.510 & 33.650 & 1380.000 \\
\hline \multicolumn{4}{|c|}{ Motorleistung in $\mathrm{kW}$} & $\begin{array}{c}487 \\
(662)\end{array}$ & $\begin{array}{c}16015 \\
(21780)\end{array}$ & $\begin{array}{c}17885 \\
(24324)\end{array}$ & $\begin{array}{c}7069 \\
(9614)\end{array}$ & $\begin{array}{c}2464 \\
(3351)\end{array}$ & $\begin{array}{c}44920 \\
(61091)\end{array}$ \\
\hline \multicolumn{4}{|c|}{ Motorleistung in kW (PS) je 100 ha LF } & \multicolumn{6}{|c|}{$278(378)$} \\
\hline \multicolumn{10}{|c|}{ Schlepper in den Betrieben bestimmter Gruppengrößen in den Jahren 1996-2002 } \\
\hline Jahr & bis 1 & $1-2$ & $2-5$ & $5-10$ & $10-1$ & $15-20$ & $20-5$ & $50-100$ & $\geq 100$ \\
\hline 1996 & 38100 & 66316 & 241317 & 3904 & 2420 & 12316 & 1319 & 14245 & 5323 \\
\hline 2002 & 53336 & 91401 & 272147 & 3527 & 2160 & 12305 & 1821 & 31267 & 42376 \\
\hline $96-02 \%$ & +40 & $+37,8$ & $+12,8$ & $-8,6$ & -11 & $-0,1$ & +38 & $+119,5$ & $-23,4$ \\
\hline
\end{tabular}

Der gesamte Mähdrescherbestand bezifferte sich im Jahr 2002 auf 123.835 Mähdrescher. Dabei ist auffallend, dass die Mehrheit der Maschinen sich in den kleinen Betrieben befindet. Auf die Betriebe des Größenbereichs von 1-15 ha LF entfallen ca. $48 \%$ des gesamten Mähdrescherbestandes. Lediglich $11 \%$ aller Mähdrescher gehören den Betrieben mit einer Flächenausstattung von mehr als 50 ha LF. 
Die Ergebnisse des letzten landwirtschaftlichen Verzeichnisses lassen den Schluss zu, dass die Maschinen nur in geringem Maße ausgelastet werden. Wie die Tabelle 4-9 beleuchtet, wird ein Schlepper durchschnittlich 350 h pro Jahr eingesetzt. Bei einer in den polnischen Maschineneinsatzkalkulationen nach IBMER (Institut für Bauwesen Mechanisierung und Elektrifizierung der Landwirtschaft) unterstellten AfA-Dauer von 20 Jahren entspricht es ungefähr $58 \%$ der erforderlichen Auslastung. Im Fall von Mähdreschern entspricht die tatsächlich erbrachte Leistung von 64 ha pro Jahr ca. 52 \% der erforderlichen Ausstattung.

Tabelle 4-9: Die Auslastung der Schlepper und der Mähdrescher in den Jahren 1996-2002 20

\begin{tabular}{|l|c|c|}
\hline & 1996 & 2002 \\
\cline { 2 - 3 } Zahl der Mähdrescher & 97058 & 123835 \\
Mähdruschfläche in 1000 ha LF & 9049 & 8778 \\
Potenzielle Arbeitsleistung eines Mähdreschers in ha/Jahr; h/Jahr & $93 ; 124$ & $71 ; 89$ \\
Einsatzfläche der Mähdrescher in \% der ges. Mähdruschfläche & $85 \%$ & $90 \%$ \\
geschätzte Auslastung in ha/ Jahr; h/Jahr & $79 ; 105$ & $64 ; 80$ \\
erforderliche Auslastung bei 20-jähriger Abschreibung & 110 ha /Jahr; 150 h/Jahr \\
\cline { 2 - 3 } Zahl der Schlepper & 1297908 & 1364579 \\
geschätzte Auslastung eines Schleppers & $390 \mathrm{~h}$ & $350 \mathrm{~h}$ \\
\cline { 2 - 3 } erforderliche Auslastung bei 20-jähriger Abschreibung & \multicolumn{2}{|c|}{$600 \mathrm{~h}$} \\
\hline
\end{tabular}

Die eingesetzten Maschinen zeichnen sich durch ein relativ hohes Alter und einen starken Abnutzungsgrad aus. Nach Schätzungen von IBMER beträgt das Alter der in den landwirtschaftlichen Betrieben vorhandener Schlepper im Durchschnitt 23 Jahre. Dabei wird der Abnutzungsgrad auf 70 \% geschätzt. Ungefähr $77 \%$ aller Schlepper in der Landwirtschaft werden bereits länger als 10 Jahre genutzt. Die übrigen $33 \%$ der Schlepper werden schon seit über 16 Jahren gebraucht. Das Alter eines Mähdreschers beläuft sich auf 21 Jahre, dabei beträgt der Abnutzungsgrad ca. 75 \% (vgl. MUZALEWSKI 2004).

Der Zustand der technischen Ausstattung in den landwirtschaftlichen Betrieben wir durch die Ersatzinvestitionen nicht verbessert. Dies liegt zumindest teilweise daran, dass die Investitionen zum großen Teil in Anschaffung von gebrauchten oft sehr stark abgenutzten Maschinen bestehen. Dabei handelt es sich im Wesentlichen um den Import von gebrauchten Maschinen aus den westlichen Nachbarländern. So entfallen auf die insgesamt 82.100

\footnotetext{
${ }^{20}$ Die in dieser Tabelle dargestellten Zahlen gelten als allgemeine Schätzungen und sind nicht empirisch bestätigt.
} 
Schlepperinvestitionen aus den Jahren 1996-2002 nahezu $40 \%$ auf gebrauchte importierte Schlepper (vgl. GUS 2002).

\subsection{Kooperationsformen}

Die Idee der Kooperation ist in der polnischen Landwirtschaft generell kaum verbreitet. Als ein wesentlicher Grund hierfür werden die negativen Erfahrungen aus den letzen Jahrzehnten genannt, in denen der private Sektor permanent den Versuchen der Verstaatlichung oder Vergesellschaftung unterworfen war. Unter den Bedingungen der zentral gesteuerten Planwirtschaft entwickelten sich keine freiwilligen Zusammenschlüsse, sei es horizontaler oder vertikaler Kooperation. Alle staatlich initiierten Kooperationsformen hatten zum Ziel, den Übergang zur kollektiven Wirtschaftsweise vorzubereiten. Im Folgenden wird ein Überblick über praktizierte Kooperationsformen der Einzelbetriebe, beginnend in den sozialistischen Zeiten bis zur Gegenwart, dargestellt ${ }^{21}$.

\section{Landwirtschaftliche Zirkel}

Im Bereich der Mechanisierung kam eine besondere Bedeutung den sog. landwirtschaftlichen Zirkeln (LZ) zu. Ihre Tätigkeit bestand zunächst in der Organisation von Maschinengemeinschaften, mit der Aufgabe verschiedene Dienstleistungen mithilfe von eigenem Maschinenpark in einzelnen landwirtschaftlichen Betrieben zu verrichten. An den Gemeinschaften, die unter starker Förderung des Staates entstanden, waren einzelne Landwirte durch einen bestimmten Kapitalbeitrag beteiligt. Die Maschinengemeinschaften wurden durch einen von den beteiligten Landwirten gewählten Ausschuss geleitet. Die gemeinsamen Maschinen wurden durch ein fest eingestelltes Personal bedient. Die Abrechung der in den landwirtschaftlichen Betrieben erbrachten Leistungen erfolgte, bei Berücksichtigung der Anteile einzelner Mitglieder, auf monetärer Basis. Da diese Kooperationsform die ihr durch die Regierung zugedachte Funktion generell nicht erfüllte, wurde sie Anfang der siebziger Jahre organisatorisch in die sog. Genossenschaften der landwirtschaftlichen Zirkel (GLZ) eingegliedert. Dabei handelt es sich um eine den LPG ähnliche Einrichtung, die auf solchen Flächen wirtschafteten, die wegen starker Zersplitterung nicht in die Staatsgüter oder LPG einverleibt worden waren. Mit der Förderung der GLZ, die der Voraussetzung weiterer Produktionszentralisierung Rechnung tragen sollten, wurden die LZ einzelner Dörfer ihrer Selbständigkeit enthoben.

\footnotetext{
${ }^{21}$ Für eine ausführliche Studie der Kooperationsformen in der polnischen Landwirtschaft zu den sozialistischen Zeiten wird auf Hegenbarth 1973 und 1977 verwiesen
} 
Nach zahlreichen Umwandlungen ist die Organisation der LZ auch heute in der polnischen Landwirtschaft unter dem Dach des Landesverbandes der Landwirtschaftsringe und landwirtschaftlichen Organisationen (KZKiOR) präsent. Wenngleich der Mechanisierung in den heutigen Aufgaben dieser Organisation eine eher marginale Bedeutung zukommt, bleiben in bestimmten Regionen des Landes nach wie vor einzelne GLZ existent. Dabei handelt es sich um Einrichtungen, die im Rahmen des übrig gebliebenen Maschinenparks bestimmte Lohnarbeiten im Bereich von Mähdrusch, Pflanzenschutz oder Düngerstreuen anbieten

Als eine Alternative für die Mechanisierung im Rahmen der Maschinengemeinschaften oder GLZ entstanden in den kommunistischen Zeiten die sog. Einzelbauerngemeinschaften, die sich in ihrer Tätigkeit meist auf die im eigenen Ermessen festgelegten Regeln stützten. In einer Gemeinschaft mussten zumindest drei selbständige auf eigenen Flächen wirtschaftende Landwirte vereint werden. Die Landwirte erhielten finanzielle Hilfe in Form von zinsverbilligten Krediten oder u.U. verlorenen Zuschüssen, die mit Vorrecht bei Maschinenanschaffung verbunden waren ${ }^{22}$. Den Mitgliedern der Maschinengemeinschaften oblag es, die tatsächliche Ausnutzung der Maschinen anhand vorgenommener Abschreibung den LZ, die damals auch eine Monopolposition im Handel mit Landmaschinen hatten, nachzuweisen. Nachdem die Maschinen völlig abgeschrieben waren, übergingen sie ins Eigentum der Landwirte. In diesen Gemeinschaften waren meistens Schlepper, Pflanzenschutzspritzen und Düngerstreuer eingesetzt. Das Hauptziel der Einzelbauergemeinschaften, durch Bildung des gemeinsamen unteilbaren Eigentums die privaten Betriebe allmählich in den Kooperationsprozess einzubeziehen, scheiterte in der Regel daran, dass die Interessen der Gemeinschaftsmitglieder oft sehr unterschiedlich waren. Auf diesem Grund kam es zu Missverständnissen, die die Zusammenarbeit unmöglich machten.

\section{Maschinenringe}

Die Idee der Propagierung der überbetrieblichen Maschinennutzung wurde nach dem Umstrukturierungsprozess erneut von IBMER aufgenommen. In den Jahren 1992-1997 wurden Versuche unternommen bei Inanspruchnahme der Fördermittel aus dem PHARE- und FAPA- Programm unter Beteiligung der Experten aus KTBL (Kuratorium für Technik und

\footnotetext{
22 In den siebziger Jahren wurde durch einen Beschluss des Zentralverbandes der LZ ermöglicht, den Einzelbauerngemeinschaften neue und gebrauchte Maschinen unter besonderen Bedingungen zu überweisen. $\mathrm{Zu}$ den Zeiten in denen die Nachfrage nach Landmaschinen das Angebot überstieg und der Verkauf streng reglementiert war, ermöglichte diese Regelung den Einzelbauerngemeinschaften im Gegenteil zu Familienbetrieben eine Sonderstellung bei Maschinenanschaffung zu genießen
} 
Bauwesen in der Landwirtschaft), in bestimmten Regionen Polens einige MR nach dem deutschen Vorbild zu etablieren. In der ersten Phase der Popularisierung dieser Form der Zusammenarbeit ist es gelungen, $14 \mathrm{MR}$ zu gründen. Rund $10 \mathrm{MR}$ entstanden in den südlichen Regionen Polens. Auf einen MR entfielen im Schnitt 31 Mitglieder. Die Funktion des Geschäftsführers wurde ehrenamtlich von einer unter den Mitgliedern ausgewählten Person wahrgenommen. Nur in einem der MR wurde auf einer halben Stelle ein Geschäftsführer beschäftigt. Die Finanzierung der MR erfolgte mittels der Mitgliedsbeiträge und zusätzlicher Zuschüsse von Gemeinden. Lediglich in $2 \mathrm{MR}$ wurde eine Provision für erbrachte Dienstleistungen erhoben (vgl. OLSZEWSKI, MuZALEWSKI 1999, S. 5).

Wenngleich die Idee der Zusammenarbeit im Rahmen der MR in den ersten Jahren gute Resonanz gefunden hat, wurde eine Weiterentwicklung dieser Kooperationsform in den Folgejahren wesentlich gebremst. Direkt nach der Einstellung der intensiven Propagierung der MR ist eine Rückentwicklung zu verzeichnen. Im Jahr 2003 funktionierten nur 9 der ursprünglich gegründeten MR. Dabei handelt es sich um Einrichtungen, die wegen ihrer Funktion und Struktur mit den deutschen MR nicht verglichen werden können. Die meisten Zusammenschlüsse haben einen informellen Charakter. Sowohl die Dienstleistung als auch die diesbezügliche Kostenabrechung werden direkt zwischen einzelnen Landwirten abgesprochen, ohne dabei dritte Personen in Anspruch $\mathrm{zu}$ nehmen (vgl. MuZALEWSKi 2003, S. 10 ff.).

Das Missfallen bei der Verbreitung dieser Form der Zusammenarbeit ist auf vielfältige Gründe zurückzuführen. In der polnischen Landwirtschaft fehlt es an positiven Erfahrungen mit Kooperationen. Relativ wenig verbreitet ist die Überzeugung von der Vorzüglichkeit der überbetrieblichen Zusammenarbeit. Aufgrund der individualistischen und den Eigenbesitz wahrenden Motivationen, wird in den landwirtschaftlichen Betrieben Eigenmechanisierung angestrebt. Die in vielen Betrieben eingesetzten Maschinen sind wegen der technischen Abnutzung und des damit einhergehenden hohen Ausfallrisikos für den überbetrieblichen Einsatz nicht geeignet. Weiterhin ist die Akzeptanz der Landwirte für die organisierten Formen der Zusammenarbeit gering. Die Schaffung oder Wahrnehmung von Führungsfunktionen stoßen auf dem Lande i.d.R. auf Abneigung. Hinzu kommen auch die Probleme organisatorischen Charakters. Zum Zeitpunkt der Gründung der MR fehlte es an rechtlichen Grundlagen für die Förderung der Entwicklung der Zusammenarbeit. Erst im Jahr 2000 wurde ein Gesetz über die Organisationen der Landwirte erlassen (siehe hierzu S. 92). Die Tätigkeit der MR, die von Anfang an als selbständige Wirtschaftssubjekte agieren, war 
mit der Buchführungspflicht verbunden. Der diesbezügliche große Aufwand sowie das restriktive Vorgehen der Finanzbehörden gegenüber MR, die dieser Pflicht nicht nachgingen, war ein weiteres wesentliches Hindernis für die Entwicklung dieser Form der Zusammenarbeit. Nicht ohne Bedeutung ist, dass die örtlichen Behörden und landwirtschaftlichen Beratungsstellen (LBS) nur in der ersten Phase der Propagierung der MR in dem Prozess aktiv mitwirkten. Es ist auf Dauer nicht gelungen, sie für die Fortsetzung der Maßnahmen zur weiteren Verbreitung dieser Form der Zusammenarbeit zu gewinnen. Schließlich ist als Ursache für das Scheitern der getroffenen Propagierungsmaßnahmen, die generell immer noch schwach entwickelte Infrastruktur der ländlichen Regionen zu nennen (Muzalewski 2005).

\section{Branchenkreditprogramm}

Für die Förderung der Entwicklung überbetrieblicher Zusammenarbeit beim Maschineneinsatz wurde im Jahr 1997 im Rahmen des sog. Branchenprogramms eine Kreditlinie für die Maschineninvestitionen gebildet. Es handelt sich dabei um zinsverbilligte Kredite, die von bestimmten Banken in Zusammenarbeit mit der Agentur für Umstrukturierung und Modernisierung der Landwirtschaft (ARiMR) angeboten werden. Diese Förderung ist gerichtet sowohl an einzelne Landwirte, die bestimmte Maschinen gemeinsam anschaffen und anschließend in eigenen Betrieben einsetzten, als auch an Unternehmen die Lohmaschinenleistungen für andere Betriebe $\mathrm{zu}$ erbringen beabsichtigen. Mit diesem Kredit können Investitionen in neue und gebrauchte Maschinen finanziert werden. Letzteres gilt allerdings nur für Maschinen die höchstens drei Jahre alt sind. Im Rahmen des Programms werden im Wesentlichen die Anschaffungen von Schleppern, Mähdreschern, Anhängern und Transportwagen finanziert. Das Programm umfasst zusätzlich die Errichtung oder Modernisierung von bestimmten Anlagen zur Verarbeitung und Lagerung von landwirtschaftlichen Erzeugnissen. Hierzu zählen u.a. Sortieranlagen für Obst und Gemüse sowie Trocknungs- und Lagerungsanlagen für Getreide. Bei der ersten Adressatengruppe, also einzelnen Landwirten die eine Maschinengemeinschaft gründen, sind die Grundlagen geplanter Zusammenarbeit in Form eines Zivilvertrages festzulegen. In dem Vertrag müssen folgende Punkte bestimmt werden:

- Gegenstand der gemeinsamen Nutzung,

- aus der Finanzierung resultierenden Anteile an dem Gegenstand,

- Dauer und Umfang der geplanten Zusammenarbeit, 
- Art und Frist der mit den gemeinsamen Maschinen zu verrichteten Arbeiten,

- genaue Regeln zur Abrechung, der während der Nutzungszeit anfallenden Kosten.

Die Inanspruchnahme des Kredits setzt eine Anerkennung der geplanten Investition durch eine örtliche LBS voraus. Somit steht sie nur den Betrieben zu, die als existenzsicher beurteilt werden. Aus den jährlichen Berichten der ARiMR geht hervor, dass die meisten Kreditanträge im ersten Jahr nach der Aktivierung des Programms gestellt wurden. In den Folgejahren nahm die Zahl der Anträge tendenziell ab. Bis Ende 2004 wurden insgesamt 1.911 Kredite vergeben (siehe Tabelle 4-10). Dies entspricht ca. $77 \%$ aller von örtlichen Beratungsstellen positiv begutachteten Anträge. Über die Hälfte der Kredite entfällt auf Unternehmen der Lohnmaschinenleistung. Sowohl die Anzahl als auch die Höhe der gewährten Kredite ist regional unterschiedlich. Dabei ist die höchste Aktivität für die landwirtschaftlichen Betriebe der westlichen Gebiete des Landes kennzeichnend. Am wenigsten Kredite wurden in den südlichen und südöstlichen Regionen aufgenommen. Insgesamt kann festgehalten werden, dass die Empfänger der Förderung v.a. die größeren Betriebe sind, die zum einen das erforderliche Eigenkapital aufbringen können und zum anderen eine entsprechende Maschinenauslastung sichern. Unter den Bedingungen der kleineren Betriebe können die Voraussetzungen für die Inanspruchnahme der Kredite oft nicht erfüllt werden. Dabei handelt es sich u.a. um die Sicherung der erforderlichen Auslastung der Maschinen. Die Kreditbedingungen setzen nämlich voraus, dass der aus der Produktionsstruktur resultierende Arbeitsbedarf in den Mitgliedsbetrieben eine ausreichende Auslastung der Maschinen gewährleistet. Die Maschinen sollen nicht in dritten Betrieben eingesetzt werden. Dem niedrigeren Leistungsbedarf in den kleineren Betrieben kommt auch die beschränkte Möglichkeit der Anschaffung gebrauchter Maschinen kaum entgegen.

Tabelle 4-10: Kredite des Branchenprogramms für gemeinsame Maschinennutzung in den Jahren 1997-2004

\begin{tabular}{|c|c|c|c|c|c|c|}
\hline \multirow{2}{*}{ Jahr } & \multicolumn{2}{|c|}{ Anzahl der von Beratungsstellen anerkannten Anträge } & \multicolumn{2}{c|}{ Gewährte Kredite } \\
\cline { 2 - 7 } & Insgesamt & Gemeinschaften & $\begin{array}{c}\text { Lohn- } \\
\text {-unternehmen }\end{array}$ & $\begin{array}{c}\text { Spezielle } \\
\text { Bereiche }\end{array}$ & $\begin{array}{c}\text { Anzahl } \\
\text { Betrag pro } \\
\text { Kredit } \\
\text { in 1000 ZL }\end{array}$ & $\begin{array}{c}\text { ZL } \\
1997\end{array}$ \\
\hline 1998 & 303 & 233 & 380 & 1 & 476 & 133,2 \\
1999 & 276 & 148 & 155 & 0 & 191 & 170,1 \\
2000 & 351 & 123 & 151 & 2 & 214 & 192,0 \\
2001 & 338 & 180 & 182 & 1 & 268 & 249,2 \\
2002 & 345 & 212 & 134 & 1 & 298 & 227,3 \\
2003 & 169 & 108 & 61 & 0 & 145 & 204,3 \\
2004 & 81 & 52 & 28 & 1 & 74 & 233,4 \\
\hline insgesamt & 2477 & 1224 & 1243 & 10 & 1911 & 215,2 \\
\hline in \% & 100 & 49,4 & 50,2 & 0,4 & 77,1 & - \\
\hline
\end{tabular}




\section{Erzeugergruppen}

Eine Rolle in dem von Ministerium für Landwirtschaft und ländliche Entwicklung (MRiRW) realisierten Entwicklungsprogramm der Landwirtschaft spielt die Förderung der Erzeugergruppen der Pflanzen- und Tierproduktion. Rechtliche Grundlagen hierzu schafft das Gesetz über landwirtschaftliche Erzeugergruppen und Verbände aus dem Jahr 2000 (vgl. Dz.U. 00.88.9983). Hier wurden zunächst die Ziele der Erzeugergruppen definiert. Dabei handelt es sich um die Anpassung der landwirtschaftlichen Produktion an die Marktbedingungen, die Bündelung des Angebots hergestellter Erzeugnisse, die Verbesserung der Produktionseffizienz unter Berücksichtigung der Qualität und Quantität der Erzeugnisse sowie um die Aspekte des Umweltschutzes. Gemäß ursprünglicher Regelung konnten den Status der Erzeugergruppen nur die Zusammenschlüsse führen, deren Umsätze aus dem gemeinsamen Verkauf der Erzeugnisse insgesamt $200.000 \mathrm{ZL} / \mathrm{Jahr}$ überstiegen. Diese Bestimmung wurde im Jahr 2003 abgeschafft, dabei wurden die minimalen Produktionsmengen einer Erzeugergruppe unter Berücksichtigung der betriebsstrukturellen Unterschiede bestimmter Regionen festgeschrieben (vgl. Dz.U. Nr. 03.138.1319).

Eine Erzeugergruppe muss als selbständiges Wirtschafssubjekt in Form einer Gemeinschaft, einer Gesellschaft oder eines Vereins ins Handelsregister eingetragen werden. Es wird vorausgesetzt, dass eine Erzeugergruppe zumindest 5 Mitglieder zählt, von denen keiner in der Mitglieder- bzw. Gesellschafterversammlung über mehr als $20 \%$ aller Stimmen verfügt. Der Umfang der Zusammenarbeit ist schriftlich in einem Vertrag festzulegen. In diesem Vertag können u.a. die Bedingungen der gemeinsamen Maschinennutzung bestimmt werden. Die Tätigkeit der Erzeugergruppen wird finanziell gefördert. Die Förderung, die teils aus dem europäischen Ausrichtungs- und Garantiefonds für die Landwirtschaft (EAGFL) und teils aus dem polnischen Staatshaushalt stammt, ist ein Bestandteil des polnischen Planes für die Entwicklung des ländlichen Raums (PROW) für die Jahre 2004-2006. Die Inanspruchnahme der Fördermittel setzt die Eintragung der Erzeugergruppe in ein von ARiMR geführtes Register voraus. Die Förderung kann höchstens fünf Jahre erfolgen. Dabei wird ihre Höhe für jedes einzelne Jahr prozentual zu dem Umsatz der Erzeugergruppe berechnet. Hierzu ist auf die Tabelle 4-11 zu verweisen. 
Tabelle 4-11: Förderungshöhe für Erzeugergruppen nach Jahren und realisiertem Umsatz

\begin{tabular}{|c|c|c|c|}
\hline Jahr & $\begin{array}{c}\text { in \% von gesamten } \\
\text { Einnahmen bis zu } \\
100.000 \text { EUR }\end{array}$ & $\begin{array}{c}\text { in \% von gesamten } \\
\text { Einnahmen über } \\
100.000 \text { EUR }\end{array}$ & $\begin{array}{c}\text { Obergrenze der Förderungsquote } \\
\text { in EUR }\end{array}$ \\
\hline 1 & 5 & 2,5 & 100000 \\
\hline 2 & 5 & 2,5 & 100000 \\
\hline 3 & 3 & 2 & 80000 \\
\hline 4 & 4 & 1,5 & 60000 \\
\hline 5 & 2 & 1,5 & 50000 \\
\hline
\end{tabular}

Quelle: ARiMR 2005, S.4 ff.

Im Jahr 2006 gab es in ganz Polen insgesamt 120 registrierte Erzeugergruppen mit 17.500 Mitgliedern. Am stärksten vertreten sind hier die Erzeugergruppen der Schweinehalter und der Obst- und Gemüseanbauer. In einer Erzeugergruppe der Schweinehalter werden im Schnitt 70 Personen vereinigt. Auf eine Erzeugergruppe der Obst- und Gemüseanbauer entfallen ungefähr 37 Personen. Eine ähnliche Anzahl der Mitglieder kennzeichnet die Erzeugergruppen der Getreideanbauer. So zählt ein Zusammenschluss der Getreideanbauer durchschnittlich 35 Mitglieder. Einen Überblick über ausgewählte Erzeugergruppen bestimmter Woiwodschaften gibt die Tabelle 4-12.

Tabelle 4-12: Anzahl ausgewählter Erzeugergruppen in bestimmten Woiwodschaften

\begin{tabular}{|c|c|c|c|c|c|c|c|c|}
\hline Erzeugnisse & Getreide & Ölfrüchte & $\begin{array}{c}\text { Hack- } \\
\text { früchte }\end{array}$ & Rinder & Schweine & Geflügel & $\begin{array}{c}\text { Obst und } \\
\text { Gemüse }\end{array}$ & Hopfen \\
\hline Niederschlesien & 5 & 2 & 0 & 0 & 0 & 0 & 0 & 1 \\
\hline Kujawien- Pommern & 5 & 2 & 2 & 1 & 6 & 1 & 0 & 0 \\
\hline Lublin & 0 & 0 & 0 & 0 & 0 & 1 & 3 & 6 \\
\hline Lebuser- Land & 1 & 0 & 0 & 1 & 0 & 1 & 0 & 0 \\
\hline Lodz & 1 & 0 & 0 & 0 & 0 & 0 & 0 & 0 \\
\hline Kleinpolen & 0 & 0 & 0 & 0 & 2 & 2 & 6 & 2 \\
\hline Masowien & 0 & 0 & 1 & 1 & 2 & 0 & 5 & 0 \\
\hline Oppeln & 2 & 6 & 0 & 0 & 0 & 1 & 0 & 0 \\
\hline Vorkarpaten & 1 & 1 & 0 & 0 & 2 & 1 & 1 & 0 \\
\hline Podlachien & 0 & 0 & 0 & 0 & 0 & 2 & 1 & 0 \\
\hline Pommern & 1 & 1 & 0 & 1 & 1 & 0 & 0 & 0 \\
\hline Heiligkreuz & 0 & 0 & 0 & 0 & 0 & 1 & 1 & 1 \\
\hline Ermland- Masuren & 1 & 0 & 0 & 0 & 1 & 0 & 2 & 0 \\
\hline Großpolen & 0 & 0 & 0 & 1 & 13 & 1 & 3 & 1 \\
\hline Westpommern & 1 & 0 & 1 & 1 & 0 & 0 & 0 & 0 \\
\hline Insgesamt & 18 & 12 & 4 & 6 & 27 & 11 & 22 & 11 \\
\hline
\end{tabular}

Quelle: MRiRW 2006

\section{Lohnunternehmen}

Im Jahr 2005 wurden in Polen fast 12.000 landwirtschaftliche Lohnunternehmen mit der Dienstleistung im Maschinenbereich verzeichnet (vgl. IERiGZ 2006) ${ }^{23}$. Es handelt sich dabei

\footnotetext{
${ }^{23}$ Da es sich bei dieser Datengrundlage zum großen Teil um Schätzungen und nicht klar abgegrenzte Definition der Lohnunternehmen handelt, kann die hier dargestellte Zahl nur mit Vorbehalten interpretiert werden.
} 
um die Unternehmen des Landmaschineneinsatzes inkl. Transports, der Pflege und Instandsetzung der luf Maschinen. Diese Gruppe besteht fast ausschließlich (99,7 \%) aus den privaten Unternehmen. Es dominieren Einheiten mit 1 bis 2 AK. Dabei handelt es sich zum großen Teil um landwirtschaftliche Betriebe, die im Rahmen der vorhandenen freien Kapazitäten bestimmte Arbeiten in anderen Betrieben verrichten. Als eine zweite einheitliche Gruppe, die in den letzten Jahren an Bedeutung gewinnt, können hier die Maschinenhändler betrachtet werden. Dies betrifft v.a. kleinere Unternehmen, die mit den vorhandenen, zumeist gebrauchten Maschinen bestimmte Dienstleistungen erbringen.

In der Verbreitung der statistisch erfassten LU sind deutliche regionale Unterschiede zu erkennen. Zahlenmäßig sind die LU am stärksten im mittleren und südlichen Teil Polens vertreten, also in Regionen, in denen kleine und sehr kleine Betriebe vorherrschen. Relativ niedrig ist die Anzahl der LU in den nördlichen Regionen. Die regionalen Unterschiede in der Verbreitung der LU kommen auch zum Ausdruck beim Vergleich des auf einen LU entfallenden Flächenumfangs. Während in der Woiwodschaft Schlesien auf einen LU im Schnitt 500 ha entfallen, stehen einem LU in der Woiwodschaft Podlachien oder Warmien Masuren jeweils 4.500 ha bzw. 3.400 ha gegenüber (siehe Tabelle 4-13)

Tabelle 4-13: Lohnunternehmen nach Woiwodschaften im Jahr 2005

\begin{tabular}{|c|c|c|c|}
\hline Woiwodschaften & $\begin{array}{c}\text { Anzahl der LU des } \\
\text { Lohnmaschinen- } \\
\text { einsatzes }\end{array}$ & $\begin{array}{c}\text { ha LF pro LU } \\
\text { in 1 000 }\end{array}$ & $\begin{array}{c}\text { Anzahl der LU der } \\
\text { Pflege und } \\
\text { Instandsetzung der luf. } \\
\text { Maschinen }\end{array}$ \\
\hline Westpommern & 423 & 2,4 & 88 \\
\hline Großpolen & 1136 & 1,5 & 376 \\
\hline Warmien-Masuren & 316 & 3,4 & 71 \\
\hline Heiligkreuz & 277 & 2,1 & 38 \\
\hline Schlesien & 943 & 0,5 & 73 \\
\hline Pommern & 443 & 1,7 & 125 \\
\hline Podlachien & 240 & 4,5 & 53 \\
\hline Vorkarpaten & 481 & 1,6 & 61 \\
\hline Oppeln & 426 & 1,3 & 67 \\
\hline Masowien & 1504 & 1,4 & 198 \\
\hline Kleinpolen & 782 & 0,9 & 42 \\
\hline Lodz & 592 & 1,8 & 186 \\
\hline Lebuser Land & 390 & 1,7 & 167 \\
\hline Lublin & 589 & 2,5 & 116 \\
\hline Kujawien-Pommern & 938 & 1,1 & \\
\hline Niederschlesien & 682 & 1,5 & \\
\hline
\end{tabular}




\subsection{Zusammenfassung}

Die wirtschaftlichen und sozialen Änderungen, die in den neunziger Jahren in Polen vollzogen wurden, haben tiefe Defizite des polnischen Agrarsektors ans Licht gebracht. Dies betrifft sowohl die Organisation der einzelnen landwirtschaftlichen Betriebe als auch das gesamte landwirtschaftliche Umfeld. In der Landwirtschaft Polens werden nach wie vor übermäßig viele $\mathrm{AK}$ gebunden. Dies schlägt sich, in Verbindung mit einfachen Produktionsverfahren in der niedrigen Produktivität nieder.

In der Größenstruktur der landwirtschaftlichen Betriebe sind wesentliche regionale Unterschiede erkennbar. Während der südöstliche und mittlere Teil des Landes durch kleine und sehr kleine Betriebe geprägt ist, zeichnen sich die nördlichen Regionen durch einen hohen Anteil an flächenmäßig größeren Betrieben. Der Strukturwandel in der polnischen Landwirtschaft im Hinblick auf die Flächenumschichtungen und die Änderung der AKAusstattung erfolgt in einem moderaten Tempo. Die ungünstige Wirtschaftslage und hohe Arbeitslosigkeit bilden keinen Ansporn für die Abwanderung der AK aus der Landwirtschaft. Unter den bestehenden Bedingungen wird der Boden in vielen Familienhaushalten häufig als ein Garant des existenziellen Minimums betrachtet. Schließlich prägen eine niedrige Kapitalausstattung und Schwierigkeiten bei Inanspruchnahme von Fremdkapital das generell gemäßigte Investitionsverhalten.

Der Maschinenbestand in der polnischen Landwirtschaft ist stark veraltet. In den letzten Jahren nimmt der Mechanisierungsgrad der landwirtschaftlichen Betriebe zwar zu. Jedoch handelt es hier zum großen Teil um die Anschaffung von gebrauchten, oft schon sehr stark abgenutzten Maschinen. Dies trägt nicht zur Verbesserung des generell schlechten technischen Zustandes der eingesetzten Maschinen bei.

Der überbetriebliche Maschineneinsatz ist wenig entwickelt. In der jüngsten Vergangenheit wurden einige Versuche unternommen, die Idee der überbetrieblichen Zusammenarbeit zu verbreiten. Besonders interessant erscheint die Förderung der Erzeugergruppen. Das vorrangige Ziel der hier entstehenden Zusammenschlüsse ist die Marktposition der Mitgliedsbetriebe durch die Organisation des gemeinsamen Absatzes der landwirtschaftlichen Erzeugnisse zu verbessern. Die Zusammenarbeit dieser Art kann unter bestimmten Bedingungen als Ausgangspunkt zur Entwicklung der Zusammenarbeit im Maschineneinsatz betrachtet werden. Dies trifft v.a. für die Produktionsverfahren zu, in denen schlagkräftige Spezialmaschinen benötigt werden. 


\section{Untersuchung in zwei Regionen}

Das Ziel dieses Kapitels ist es, den Hintergrund für die Modernisierung der Mechanisierung in den landwirtschaftlichen Betrieben zweier Untersuchungsregionen aufzuzeigen. Daher wird das Hauptaugenmerk zunächst auf die Rahmenbedingungen der Landwirtschaft dieser Regionen gerichtet. In dem darauf folgenden Teil werden kurz die Befragungsergebnisse der landwirtschaftlichen Betriebe beider Regionen dargestellt.

\subsection{Abgrenzung der Untersuchungsregionen}

Die Untersuchungsregionen wurden unter der Prämisse ausgewählt, die regionalen Unterschiede in der Entwicklungsstruktur der polnischen Landwirtschaft möglichst weit zu erfassen. Auf diese Weise soll der Anforderung Rechnung getragen werden, die potenziellen Mechanisierungsszenarien unter unterschiedlichen Ausgangsbedingungen zu analysieren. Die erste Region ist die im nordöstlichen Teil Polens gelegene Woiwodschaft Ermland - Masuren, die zweite hingegen die südöstliche Woiwodschaft Lublin (siehe Abbildung 4-1, S. 73). Die Woiwodschaft Ermland-Masuren zeichnet sich durch eine hohe Anzahl von verhältnismäßig großen Betrieben aus. Die Betriebsgrößenstruktur dieser Region, zum großen Teil geschichtlich bedingt, ist kennzeichnend für die nördlichen Gebiete Polens. Die Landwirtschaftsstruktur in der Woiwodschaft Lublin ist demgegenüber durch flächenmäßig kleine oder sehr kleine, für den übrigen Teil Polens signifikante Betriebe geprägt. Beide Untersuchungsregionen zeichnet eine schwache Industrieentwicklung aus. Demzufolge klein ist der Anteil der Wirtschaften beider Regionen an dem Bruttoinlandsprodukt (BIP) Polens. Die Wirtschaften beider Woiwodschaften tragen jeweils ca. $3 \%$ bzw. $4 \%$ zum gesamten BIP bei. Das BIP ist in Lublin, gemessen an der Anzahl der Einwohner mit deutlichem Abstand am kleinsten in ganz Polen. Die Region Ermland Masuren rangiert bei diesem Vergleich vor Podlachien an der drittletzten Stelle.

\subsection{Charakteristik der Untersuchungsregion Ermland - Masuren}

\subsubsection{Natürliche Standortbedingungen}

Die Woiwodschaft Ermland-Masuren umfasst eine Gesamtfläche von $24.192 \mathrm{~km}^{2}$. Topographisch ist es eine hügelige Landschaft mit einem großen Anteil an Wasserflächen. Es überwiegen hier Böden von mittlerer und schlechter Qualität, d.h. sie entsprechen den Klassen III bis VI des Bonitierungssystems (siehe Übersicht 2-1, S. 7). Die mittlere Jahresdurchschnittstemperatur der Jahre 1991-2000 liegt in Ermland - Masuren bei 7,2 ${ }^{\circ}$ C. 
Die Niederschlagsmengen pendeln sich in derselben Zeitspanne auf dem Niveau von $588 \mathrm{~mm} / \mathrm{Jahr}$ ein.

\subsubsection{Betriebsgrößenstruktur}

Gemessen an der Flächenausstattung der landwirtschaftlichen Betriebe unterscheidet sich die Landwirtschaftsstruktur dieser Region wesentlich von dem Gesamtbild des Agrarsektors Polens. Im Jahr 2002 gab es in dieser Region 52.072 landwirtschaftliche Betriebe mit einer Flächenausstattung von mehr als 1 ha LF. Die durchschnittliche Flächenausstattung eines Betriebes beläuft sich auf 21,5 ha LF. Die zahlreichste Gruppe bilden die Betriebe des Größenbereichs von 1-5 ha LF. Zahlenmäßig am schlechtesten vertreten sind die Betriebe mit einer Flächenausstattung von mehr als 50 ha LF. Während auf die erste angesprochene Gruppe fast $36 \%$ aller Betriebe entfallen, verfügen lediglich 4,7\% aller Betriebe über eine Fläche von mehr als 50 ha LF. Eine Besonderheit der Betriebsstruktur der Region zeigt sich beim Vergleich der Anzahl der Betriebe bestimmter Größengruppen mit den ihnen entsprechenden Anteilen an der LF. Die zahlenmäßig größte Gruppe der Betriebe des Größenbereichs von 1-20 ha LF (77,4 \% aller Betriebe) verfügt über knapp $27 \%$ aller LF der Region. Die Betriebe mit einer Flächenausstattung von über 50 ha LF (4,7\% aller Betriebe) bewirtschaften $48,5 \%$ aller LF (siehe Tabelle 5-1).

Tabelle 5-1: Anzahl und LF der landwirtschaftlichen Betriebe der Woiwodschaft Ermland Masuren

\begin{tabular}{|c|c|c|c|c|c|c|c|c|c|}
\hline Größengruppe in ha LF & $1-2$ & $2-5$ & $5-10$ & $10-20$ & $20-30$ & $30-50$ & $50-100$ & $>100$ & gesamt \\
\hline Betriebe in 1000 & 9,6 & 9 & 7,9 & 13,8 & 5,6 & 3,7 & 1,6 & 0,8 & 52 \\
Betriebe in \% & 18,4 & 17,4 & 15,1 & 26,5 & 10,9 & 7 & 3,1 & 1,6 & 100 \\
\hline Fläche in 1000 ha LF & 13,3 & 28,8 & 58,9 & 199,2 & 137,1 & 137,3 & 107,9 & 435,6 & 1118,1 \\
Fläche in \% & 1,2 & 2,6 & 5,3 & 17,8 & 12,3 & 12,3 & 9,6 & 38,9 & 100 \\
\hline
\end{tabular}

\subsubsection{Bodennutzung und Struktur der Nutztierbestände}

Von der gesamten LF der Woiwodschaft Ermland - Masuren (1,1 Mio. ha) entfallen ca. 69 \% auf die Ackerflächen und 30,8 \% auf das Dauergrünland. Eine geringe Bedeutung kommt hier dem Gartenbau zu. So werden insgesamt 2.800 ha (0,2 \% aller LF) dieser Nutzungskategorie zugeordnet. Kennzeichnend für die Flächenstruktur dieser Region ist ein hoher Anteil an Brach- und Ödland. Es wurden im Jahr 2002 insgesamt 116.800 ha oder 17,8 \% des Ackerlandes nicht bewirtschaftet. 
Innerhalb des Ackerlandes nimmt der Getreideanbau eine besondere Stellung ein. Es werden ca. $82 \%$ des Ackerlandes für Getreideanbau beansprucht. Davon entfallen ca. $30 \%$ auf Weizen und ca. 12 \% auf Roggen. Die zweitgrößte Bedeutung kommt in der Region dem Anbau von Raps- und Rübsen zu. So werden für diese Kulturen insgesamt 33.402 ha LF (ca. $7 \%$ ) beansprucht. Weiterhin besitzen flächenmäßig eine große Bedeutung die Futterpflanzen. Diesen Anbaukulturen entsprechen 21.604 ha LF, was ca. $4 \%$ aller Ackerflächen ausmacht (siehe Abbildung 5-1).

Abbildung 5-1: Relative Anteile der Anbauflächen an dem Ackerland in Ermland - Masuren

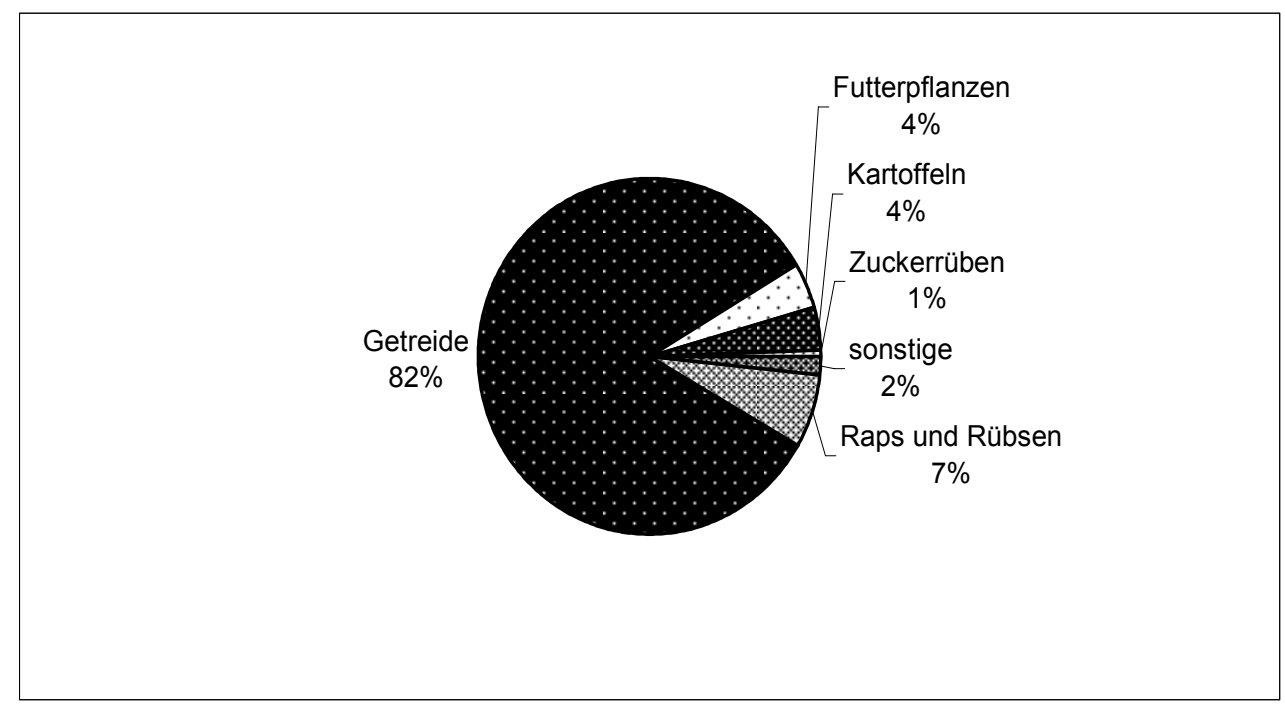

Quelle: GUS Olsztyn 2004

In allen landwirtschaftlichen Betrieben dieser Region wurden im Jahr 2002 insgesamt 391.923 Rinder gehalten. Davon entfallen ca. $46 \%$ auf Kühe. Der Rinderbestand pro 100 ha LF ist in dieser Region der höchste in ganz Polen. Auf 100 ha LF entfallen insgesamt 35 Rinder. In den Betrieben mit einer Flächenausstattung von 1-7 ha LF befinden sich 3,73 \% aller Rinderbestände. Auf die Gruppe der Betriebe von 7-20 ha LF entfallen insgesamt 32,7 \% aller Rinder. Die größten Anteile sind für die Betriebe mit einer Flächenausstattung von 20-100 ha LF signifikant. Es werden in den Betrieben dieser Gruppe insgesamt 52,7 \% aller Rinder gehalten (siehe Abbildung 5-2).

Wird das Augenmerk auf die Bestandsgröße in den einzelnen Betrieben gerichtet, so zeigt sich, dass die Rinderhaltung vorwiegend in kleinen Beständen erfolgt. Ungefähr $16 \%$ aller Betriebe haben weniger als 2 Rinder. Relativ stark vertreten sind hier Betriebe mit 5-9 und 10-19 Tieren. Die Anteile der Betriebe dieser Gruppen an allen Rinderhaltungsbetrieben 
reichen zu jeweils 19,6\% bzw. 26,6 \%. Lediglich in $3 \%$ aller Betriebe werden mehr als 50 Rinder gehalten.

Der Schweinebestand in dieser Region betrug im Jahr 2002 insgesamt 861.000 Tiere. Dies entspricht 4,6\% aller Schweinebestände Polens. Auf 100 ha LF entfallen im Schnitt 111 Tiere. Die größten Anteile an den Schweinebeständen sind für die Betriebe mit einer Flächenausstattung von über 50 ha LF kennzeichnend. Relativ wenige Schweine entfallen auf die kleinen Betriebe mit einer Flächenausstattung von 1-7 ha LF. Diesen Betrieben entsprechen ca. $4 \%$ des gesamten Schweinebestands der Region. Ähnlich wie im Fall der Rinderhaltungsbetriebe erfolgt auch die Schweinehaltung größtenteils im Rahmen von kleinen Wirtschaften. So verfügen ca. $41 \%$ aller Schweinehaltungsbetriebe über weniger als 10 Tiere. Die zahlreichste Gruppe bilden Betriebe mit 20 bis 49 Tieren. Sie machen ca. $22 \%$ aller Betriebe aus. Der Anteil der Betriebe mit 100 und mehr Tieren pendelt sich auf dem Niveau von $7,8 \%$ ein.

Abbildung 5-2: Relative Anteile bestimmter Betriebsgrößengruppen an den Rinder- und Schweinebeständen in Ermland-Masuren

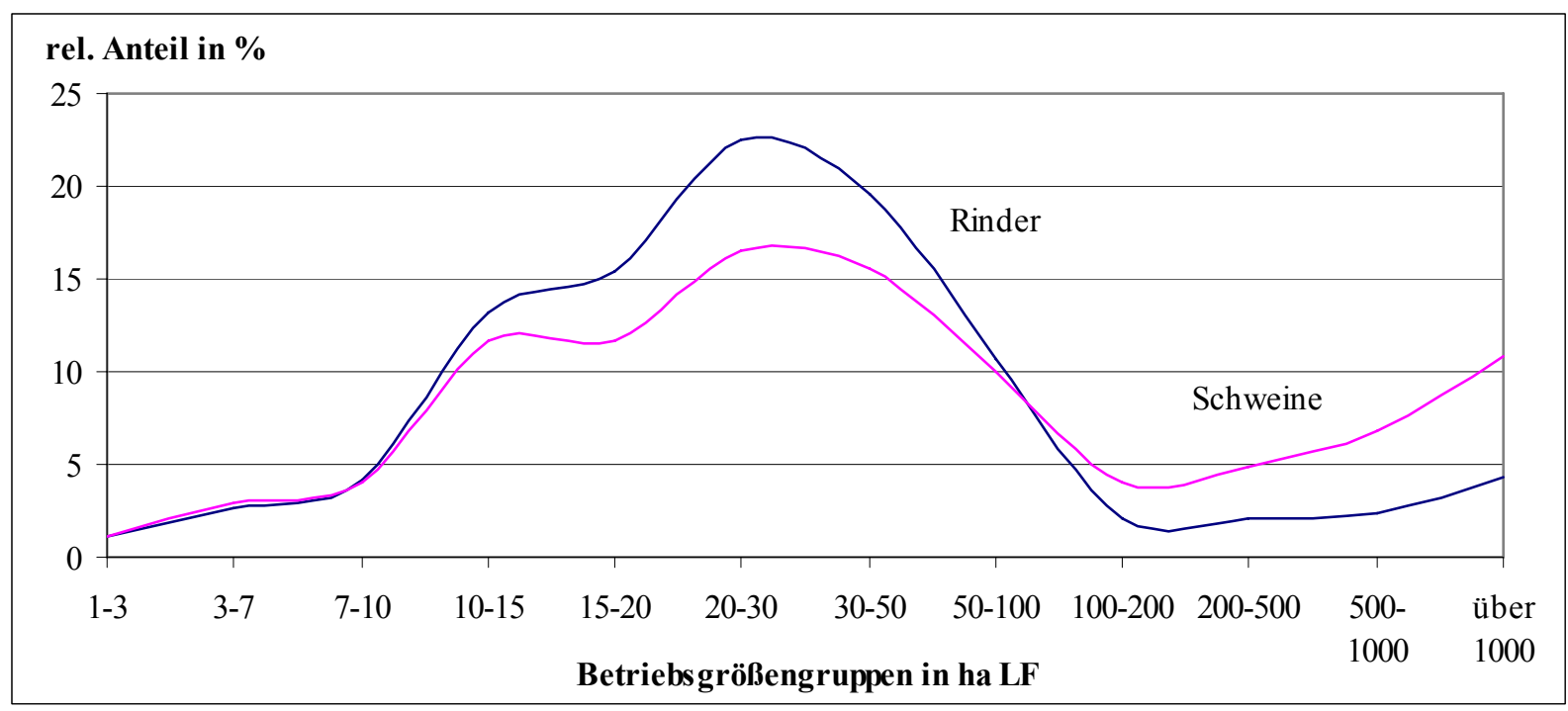

Quelle: GUS Olsztyn 2004

Es kommt in dieser Region der Geflügelhaltung eine erhebliche Bedeutung zu. Kennzeichnend ist seit einigen Jahren eine Zunahme der Geflügelbestände. Demzufolge stieg der Geflügelbestand zwischen den Jahren 1996-2002 von 0,82 Mio. auf 1,03 Mio. an. Neben der Hühnerhaltung hat in der Region auch die Putenhaltung eine wesentliche Bedeutung. So bestehen die Geflügelbestände zu 42,9 \% aus den Truthühnern. Dies entspricht nahezu 40 \% des gesamten Putenbestands Polens. 


\subsubsection{Eigentums- und Pachtverhältnisse}

Die Woiwodschaft Ermland - Masuren zählte in den sozialistischen Zeiten zu den Regionen, in denen die Verstaatlichung des Agrarsektors weit fortgeschritten war. Demgemäß niedrig war hier der Anteil von privaten Familienbetrieben. Im Vorgang der Privatisierung wurden zwar die meisten Flächen wieder in das Privateigentum überführt. Ein wesentlicher Teil der Flächen befindet sich jedoch auch heute noch in der staatlichen Verwaltung. Während im Landesdurchschnitt lediglich 5,5\% aller LN dem Staatseigentum angehören, verbleiben in dieser Region über $13 \%$ aller LN im staatlichen Sektor.

Im Vergleich zu anderen Regionen Polens hat hier die Bewirtschaftung der Pachtflächen eine große Bedeutung. Mit insgesamt 177.416 ha landwirtschaftlichen Pachtflächen weist Ermland -Masuren, hinter Großpolen, das zweitgrößte Areal der Pachtflächen in Polen auf. Ungefähr $30 \%$ aller Betriebe dieser Region verfügen über Pachtflächen. Davon entfällt ein Fünftel auf Betriebe, die ausschließlich Pachtflächen bewirtschaften. Die durchschnittliche Pachtflächengröße liegt bei 20,8 ha (vgl. GUS OLSZTYN 2004, S. 102).

\subsubsection{Bevölkerungsstruktur und AK-Ausstattung}

Auf dem Lande in der Woiwodschaft Ermland - Masuren leben insgesamt 560.000 Menschen Davon sind 195.300 Personen durch landwirtschaftliche Betriebe direkt mit der Landwirtschaft verbunden. Nahezu 57,5\% der Gruppe bilden Personen mit eigener Unterhaltungsquelle. Die übrigen 42,5 \% werden durch andere Personen finanziell unterhalten.

Im Jahr 2003 wurden im Agrarsektor in Ermland - Masuren insgesamt 66.982 Erwerbstätige gezählt. Dies entspricht 17,5\% aller Erwerbstätigen dieser Region. Für nahezu 54.000 Personen (über $96 \%$ ) bildete die Beschäftigung im eigenen Betrieb die einzige Einkommensquelle. Wie die Tabelle 5-2 zeigt, sind 91,4\% aller in den landwirtschaftlichen Betrieben tätigen Personen im Produktionsalter. Dabei entfallen 56,8 \% der Gruppe auf Personen im Alter von 18 bis 44 Jahren. 
Tabelle 5-2: Anzahl der in den landwirtschaftlichen Betrieben beschäftigten Personen nach Altersgruppen und Arbeitsumfang in Ermland - Masuren

\begin{tabular}{|c|c|c|c|}
\hline & \multirow{2}{*}{ Gesamt } & \multicolumn{2}{|c|}{ der Arbeitsumfang } \\
\cline { 3 - 4 } & & ausschließlich & hauptsächlich \\
\hline Gesamt in 1000 $^{*}$ & 56 & 54 & 2,0 \\
\hline Altersgruppen $^{24}$ & \multicolumn{3}{|c|}{ Gesamt in \% } \\
\hline Vorproduktion $_{\text {Produktion davon: }}$ & 2,1 & 2,1 & 0,4 \\
$18-44$ & 91,4 & 91,2 & 98,6 \\
$45-59 / 64$ & 56,8 & 56,5 & 64,7 \\
Nachproduktion & 34,6 & 34,7 & 33,9 \\
Quelle: GUS 2003 & 6,5 & 6,7 & 1,0 \\
\hline
\end{tabular}

\subsubsection{Arbeitsmarkt}

Im Jahr 2005 wurden in der Woiwodschaft Ermland-Masuren 158.600 Arbeitslose registriert. Dies entspricht insgesamt 28,6\% aller Erwerbsfähigen dieser Region. Die Arbeitslosenquote in der Woiwodschaft geht deutlich über den Landesdurchschnitt hinaus, zugleich gehört sie zu den höchsten in ganz Polen. Die Tatsache rührt im Wesentlichen von der schwachen Wirtschaftsentwicklung dieser Region her. Es wurden im Jahr 2004 insgesamt 108.900 Wirtschaftssubjekte registriert ${ }^{25}$. Außer den landwirtschaftlichen Betrieben handelt es sich hier v.a. um die Lebensmittelindustrie (Milch-, Fleisch- und Spirituosensektor). Eine verhältnismäßig große Bedeutung kommt in der Region auch der Holz- und Möbelindustrie zu. Alles in allem zeichnet diese Region eine relativ niedrige ökonomische Aktivität der Bevölkerung aus. Es entfallen auf 1.000 Einwohner insgesamt 75 Unternehmen. Einem Arbeitsplatzangebot stand im Jahr 2004 eine Nachfrage von insgesamt 328 registrierten Arbeitslosen gegenüber (vgl. GUS OLSZTYN 2006).

\subsection{Charakteristik der Untersuchungsregion Lublin}

\subsubsection{Natürliche Standortbedingungen}

Die Woiwodschaft Lublin ist eine im äußeren Südosten gelegene Region Polens. Die Gesamtfläche dieser Woiwodschaft umfasst $25.114 \mathrm{~km}^{2}$, davon entfallen ca. $68 \%$ auf die

\footnotetext{
${ }^{24}$ Die in der Tabelle ausgewiesenen Altersgruppen werden nach GUS wie folgt definiert: Vorproduktionsalter bis zum 17 Lebensjahr; Produktionsalter von 18 bis zum 59 Lebensjahr im Fall von Frauen bzw. von 18 bis zum 65 Lebensjahr im Fall von Männern; Nachproduktionsalter über 59 Lebensjahr im Fall von Frauen bzw. über 65 im Fall von Männern.

${ }^{25}$ Hierzu zählen juristische Personen, Organisationen ohne Rechtsform juristischer Person, natürliche Personen inkl. landwirtschaftliche Betriebe (vgl. Gesetz über öffentliche Statistik von 26.06.1995, Dz.U. Nr. 95.88.439 mit späteren Änderungen).
} 
Landwirtschaftsfläche. Kennzeichnend für diese Region ist ein relativ großer Anteil von Böden guter und sehr guter Qualität. Topographisch ist die Region als Flachland geprägt. Die mittlere Jahresdurchschnittstemperatur der Jahre 1981-2002 liegt in Lublin bei 7,4 ${ }^{\circ} \mathrm{C}$. Die Niederschlagsmengen pendeln sich in derselben Zeitspanne auf dem Niveau von $605 \mathrm{~mm} / \mathrm{Jahr}$ ein. Dabei sind Juni, Juli und September die regenreichsten Monate.

\subsubsection{Betriebsgrößenstruktur}

Im Jahr 2002 gab es in Woiwodschaft Lublin insgesamt 223.342 landwirtschaftliche Betriebe ab 1 ha LF. Die durchschnittliche Flächenausstattung eines landwirtschaftlichen Betriebes liegt unter dem Landesdurchschnitt und beträgt 6,9 ha LF. Dementsprechend befinden sich die meisten Betriebe dieser Region in den Größengruppen der geringen Flächenausstattung. Es werden nahezu $83 \%$ aller Betriebe der Größengruppe von 1-10 ha LF zugeordnet. Auf die Betriebe des Größenbereichs von 10-30 ha LF entfallen insgesamt 15,8 \% aller Betriebe. Lediglich $1,4 \%$ aller Betriebe dieser Region verfügen über mehr als 30 ha LF. Der Betriebsgrößenstruktur entsprechend, sind hier auch die Flächenanteile gestaltet. In der Bewirtschaftung der zahlenmäßig am stärksten vertretenen Betriebsgruppe mit einer Flächenausstattung bis zu 10 ha LF befinden sich ca. 50 \% aller LF dieser Region. Auf die Betriebe des Größenbereichs von 10-30 ha LF entfallen ungefähr 34,5 \% aller LF. Schließlich verfügen die Betriebe mit einer Flächenausstattung von mehr als 30 ha LF über 15,5\% aller LF (siehe Tabelle 5-3)

Tabelle 5-3: Anzahl und LF der landwirtschaftlichen Betriebe der Woiwodschaft Lublin

\begin{tabular}{|c|c|c|c|c|c|c|c|c|c|}
\hline Betriebsgröße in ha LF & $1-2$ & $2-5$ & $5-10$ & $10-20$ & $20-30$ & $30-50$ & $50-100$ & $>100$ & gesamt \\
\hline Betriebe in 1000 & 45,9 & 75 & 64,1 & 30,2 & 5,2 & 2,05 & 0,58 & 0,27 & 223,3 \\
Betriebe in \% & 20,5 & 33,6 & 28,7 & 13,5 & 2,3 & 1 & 0,3 & 0,1 & 100 \\
\hline Fläche in 1000 ha LF & 65 & 251,4 & 453,7 & 405 & 123,9 & 76 & 38 & 126,5 & 1539,5 \\
Fläche in \% & 4,2 & 16,3 & 29,4 & 26,5 & 8 & 4,9 & 2,5 & 8,2 & 100 \\
\hline
\end{tabular}

\subsubsection{Bodennutzung und Struktur der Nutztierbestände}

Von der gesamten LF der Woiwodschaft Lublin (1,5 Mio. ha) entfallen 1,2 Mio. ha oder $80 \%$ auf das Ackerland und 17,6 \% auf das Dauergrünland. Die übrigen 2,4 \% der LF werden als Gartenbau bewirtschaftet. Im Jahr 2002 wurden insgesamt 151.800 ha des Ackerlandes (ca. 10\%) nicht bewirtschaftet. Davon entfielen 114.100 auf das Brachland. Die meisten Ackerflächen werden für Getreideanbau beansprucht. Auf diese Fruchtgruppe entfallen über 
$77 \%$ des Ackerlandes. Die größte Bedeutung hat hier der Weizenanbau (ca. $34 \%$ der Getreideanbauflächen). An der zweiten Stelle rangiert Gerste (17,4\% der Getreideanbauflächen). Die drittwichtigste Getreideart ist Roggen (16,6\% aller Getreideanbauflächen). Nach wie vor nimmt der Kartoffel- sowie Zuckerrübenanbau eine bedeutende Stelle in dieser Region ein. Es werden jeweils ca. $7 \%$ bzw. $4 \%$ der Ackerflächen für den Anbau dieser Pflanzen beansprucht (siehe Abbildung 5-3).

Abbildung 5-3: Relative Anteile der Anbauflächen an dem Ackerland in Lublin

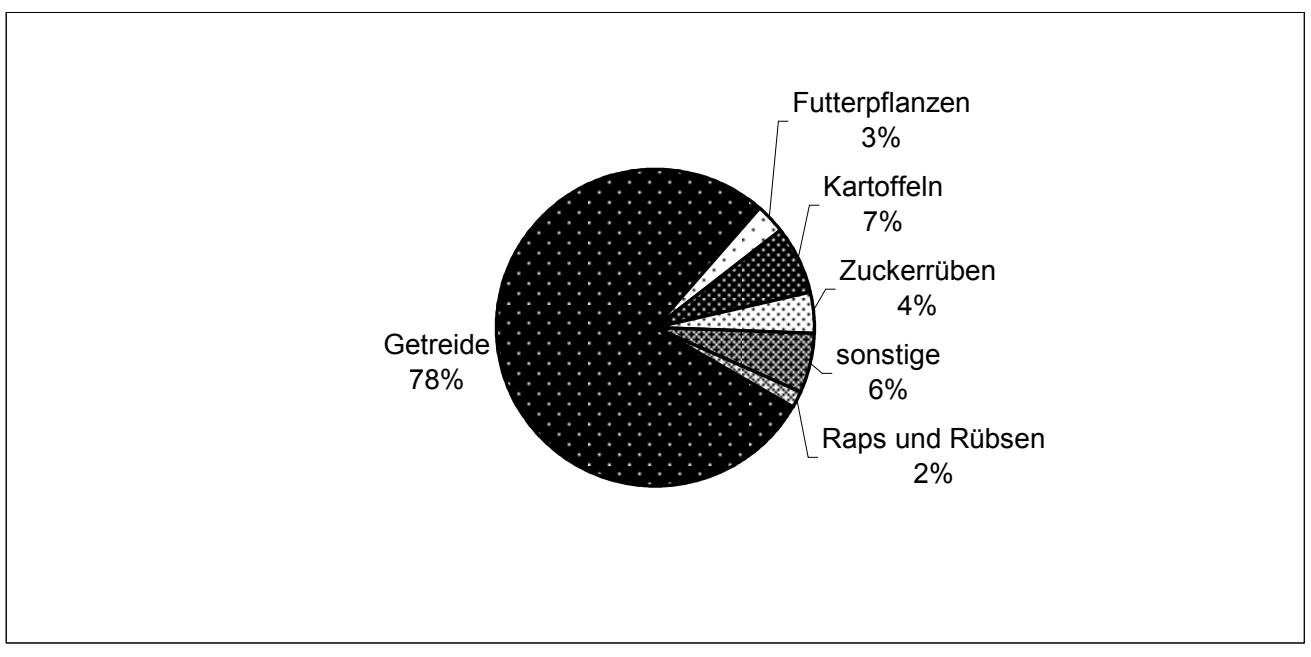

Quelle: GUS 2003

Der Rinderbestand der landwirtschaftlichen Betriebe dieser Region wurde im Jahr 2002 auf 429.934 beziffert. Davon entfielen ca. 59 \% auf Kühe (254.387). Die Zahl der Rinder dieser Region machte 7,7\% des gesamten Rinderbestandes Polens aus. Gemessen an den Flächen entfallen auf 100 ha LF im Schnitt 28 Rinder. Die Tierhaltung erfolgt gemäß der örtlichen Betriebsgrößenstruktur zum überwiegenden Teil in kleinen und sehr kleinen Familienwirtschaften. Es entfallen auf die Betriebe der Größengruppe 1-7 ha LF insgesamt 27,3\% des Rinderbestandes. Es handelt sich hier hauptsächlich um die Produktion in Beständen von 2-3 Tieren für die Deckung des eigenen Nahrungsmittelbedarfs. Die höchsten Anteile der Rinderbestände sind für die Betriebe der Größengruppe 7-15 ha LF signifikant. Es befinden sich in diesen Betrieben ca. $42 \%$ aller Rinderbestände der Region (siehe Abbildung 5-4).

Der Schweinebestand wurde im Jahr 2002 auf insgesamt 1,34 Mio. Tiere beziffert. Davon entfallen ca. $11 \%$ auf Sauen. Je 100 ha LF werden 108 Tiere gehalten. Ähnlich wie im Fall der Rinderhaltung erfolgt auch die Schweinehaltung zum großen Teil im Rahmen von kleinen 
Familienwirtschaften. Demzufolge entfallen auf die Betriebe der Größengruppe 1-7 ha LF ca. $42 \%$ des Schweinebestands.

Abbildung 5-4: Relative Anteile bestimmter Betriebsgrößengruppen an den Rinder- und Schweinebeständen in Lublin

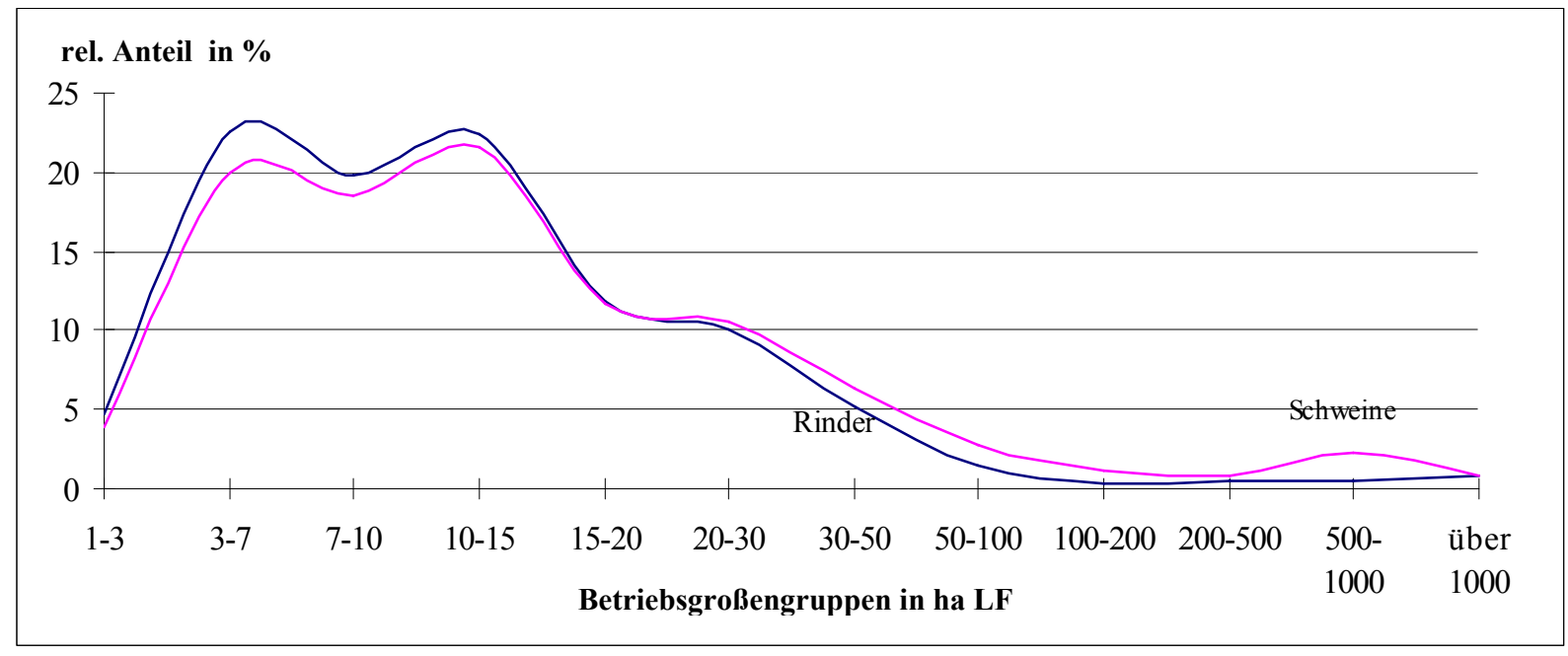

Quelle: GUS 2003

\subsubsection{Eigentums- und Pachtverhältnisse}

Das Gesamtbild der Agrarstruktur der Woiwodschaft Lublin ist durch die Betriebe des privaten Sektors geprägt. Von den gesamten 1,53 Mio. ha LF der Region befinden sich ca. $98 \%$ in der Bewirtschaftung von Privatbetrieben. Im Jahr 2002 gab es in der Region lediglich 83 Betriebe des öffentlichen Sektors.

Dominierend bleibt in dieser Region nach wie vor die Bewirtschaftung eigener Flächen. Der Flächenpacht kommt hier lediglich eine kleine Bedeutung zu. Ungefähr $18 \%$ aller Betriebe dieser Region bewirtschaften Pachtflächen. Dabei handelt es sich bei 1,2 \% um Betriebe, die über keine eigenen Flächen verfügen. Den Gegenstand der Pacht bilden generell kleine Flächen, die i.d.R. in der Nachbarschaft ohne Vertrag gepachtet werden. So lag die durchschnittliche Pachtflächengröße pro Betrieb im Jahr 2002 bei 5,6 ha LF und zählte damit zu den kleinsten in ganz Polen (vgl. GUS OLSZTYN 2004, S. 102).

\subsubsection{Bevölkerung und AK- Ausstattung}

Die Woiwodschaft Lublin zählt zu den Regionen mit den größten Anteilen der ländlichen Bevölkerung, die durch einen landwirtschaftlichen Betrieb direkt mit der Landwirtschaft verbunden ist. So zählten zu dieser Gruppe im Jahr 2002 insgesamt 863.200 Personen. Diese Gruppe besteht zu ungefähr $60 \%$ aus Personen, die über eine eigene Lebensunterhaltsquelle 
verfügen. Die restlichen $40 \%$ entfallen auf Personen, die durch andere finanziell unterhalten werden.

Im Agrarsektor der Woiwodschaft Lublin sind insgesamt 283.400 Personen beschäftigt. Dies entspricht ca. $36 \%$ aller Erwerbstätigen dieser Region. Dabei handelt es sich bei ca. $95 \%$ der Gruppe um Erwerbstätige, die ausschließlich oder hauptsächlich im eigenen landwirtschaftlichen Betrieb beschäftigt sind. Wie die Tabelle 5-4 veranschaulicht, ist hier besonders stark die Gruppe der Personen im Produktionsalter vertreten. Dabei entfallen 48,5 \% der Gruppe auf die Personen im Alter von 18 bis 44 Jahren.

Tabelle 5-4: Anzahl der in den landwirtschaftlichen Betrieben beschäftigten Personen nach Altersgruppen und Arbeitsumfang in Lublin

\begin{tabular}{|c|c|c|c|}
\hline \multirow{2}{*}{} & \multirow{2}{*}{ Gesamt } & \multicolumn{2}{|c|}{ Arbeitsumfang } \\
\cline { 3 - 4 } & & ausschließlich & überwiegend \\
\hline Gesamt in 1000 & 269,5 & 260,2 & 0,3 \\
\hline Altersgruppen & \multicolumn{3}{|c|}{ Gesamt in \% } \\
\hline Vorproduktion & 2,1 & 2,1 & 98,1 \\
Produktion davon & 84,6 & 84,1 & 65,3 \\
$18-44$ & 48,5 & 47,9 & 33,8 \\
$45-59 / 65$ & 36,1 & 36,2 & 1,8 \\
Nachproduktion & 13,3 & 13,8 & \\
\hline
\end{tabular}

\subsubsection{Arbeitsmarkt}

Im Jahr 2005 wurden in der Woiwodschaft Lublin insgesamt 167.880 Arbeitslose registriert. Diese Zahl besteht zu $54 \%$ (90.396 Personen) aus arbeitslosen Dorfeinwohnern. (vgl. GUS 2005, S. 35 ff.). Es sind hier wesentliche regionale Unterschiede des Arbeitsmarkts erkennbar. Während die Arbeitslosenquote im zentralen Teil der Woiwodschaft generell nicht über 16,8 \% hinausgeht, bleibt in östlichen Teilen ungefähr ein Viertel aller Erwerbsfähigen ohne Beschäftigung. Dieser Sachverhalt hängt mit der Entwicklung der Wirtschaftsstruktur einzelner Gebiete zusammen. Der zentrale Teil der Woiwodschaft mit der gleichnamigen Stadt Lublin zeichnet sich durch eine verhältnismäßig starke Industrialisierung aus. Allen voran handelt es sich hier um Schwer- und Chemieindustrie. Eine relativ große Bedeutung kommt in dieser Region auch der Lebensmittel- sowie der Holzindustrie zu. Wesentlich schlechter schneiden an dieser Stelle die östlichen Regionen, insbesondere die Grenzgebiete, ab. Unter den in der Woiwodschaft Lublin funktionierenden Unternehmen überwiegen relativ kleine Betriebe mit weniger als fünf Angestellten. So entsprechen dieser Gruppe ca. $93 \%$ 
aller im Jahr 2004 verzeichneten Wirtschaftssubjekte dieser Region. Die Zahl der Unternehmen mit 6 bis 25 Angestellten entspricht 3,5\% aller Unternehmen. Lediglich 105 Unternehmen $(0,08 \%)$ beschäftigen mehr als 500 Personen. Davon befinden sich 50 Unternehmen in der Stadt Lublin. Charakteristisch für diese Region ist eine relativ niedrige ökonomische Aktivität der Bevölkerung. So entfallen auf 1.000 Einwohner ca. 68 Unternehmen. Einem Arbeitsplatzangebot stand im Jahr 2004 eine Nachfrage von 246 registrierten Arbeitslosen gegenüber (vgl. GUS LUBLIN 2006).

\subsection{Auswahl typischer Betriebe}

Das Anliegen der folgenden Ausführungen ist es, zum einen die grundsätzlichen Kriterien für die Auswahl der für die weiteren Modellbetrachtungen geeigneten Betriebe aufzuzeigen und zum anderen die Befragungsergebnisse darzustellen. Bei der Auswahl der Betriebe wird das Ziel verfolgt, regionale Rahmenbedingungen und die daraus resultierenden Entwicklungsperspektiven der Mechanisierung der landwirtschaftlichen Betriebe zu identifizieren. Es wird an dieser Stelle auf die arithmetische Modellierung von Durchschnittsbetrieben verzichtet. Als Grundlage für die weiteren Überlegungen werden reale Betriebe herangezogen, die als typisch für die Untersuchungsregion eingestuft wurden. Für die Auswahl geeigneter Betriebe wird neben der Auswertung von statistischem Datenmaterial auf das Fachwissen einer Expertengruppe zurückgegriffen, die aus landwirtschaftlichen Beratern, Wissenschaftlern, Verwaltungsangestellten sowie Landwirten vor Ort bestand. Auf diese Art und Weise wird der Voraussetzung Rechnung getragen, die Gruppe der Untersuchungsbetriebe, im Hinblick auf die kardinalen Ziele des Forschungsvorhabens praxisnah auszuwählen.

Das grundsätzliche Unterscheidungskriterium bei der Bildung der Betriebsgruppen ist die Flächenausstattung. Im Fall beider Woiwodschaften werden fünf Betriebsgrößengruppen gebildet. Dabei wird das Augenmerk, abgesehen von der Gesamtzahl aller statistisch erfassten Betriebe, auf die für die Landwirtschaftsstruktur der Untersuchungsregionen signifikanten Betriebsgruppen gerichtet. Die Stichprobe besteht aus 29 Betrieben in der Region Ermland Masuren und 27 Betrieben in der Region Lublin (siehe Tabellen 5-5, 5-7 S. 110, 116). Die Verteilung der Stichprobe in den Betriebsgrößengruppen ist unregelmäßig. Es werden vielmehr die Unterschiede in der Produktionsrichtung, der AK- und Maschinenausstattung sowie die daraus resultierenden Kooperationsmöglichkeiten einzelner Betriebe beachtet. 
In der nördlichen Region finden keine Berücksichtigung Betriebe mit einer Flächenausstattung von weniger als 20 ha LF. Den Betrieben dieser Größengruppe kommt nach den Schätzungen der Experten in dieser Region keine praktische Bedeutung zu. Es handelt sich dabei zum großen Teil um Betriebe, die nur nominell existent sind, d.h. sie funktionieren als keine selbständigen Wirtschaftseinheiten und sind entweder verpachtet oder verkaufen allmählich ihre Flächen. Unter Berücksichtigung dieser Tatsache werden in der Stichprobe Betriebe des Größenbereichs 20-300 ha LF untersucht. Über diese Grenze hinausgehend wird ein, für weitere Überlegungen als wertvoll eingeschätzter Betrieb herangezogen ${ }^{26}$.

In der südlichen Region liegt der Schwerpunkt der Befragung bei kleineren Betrieben. Dementsprechend verfügen 17 Betriebe dieser Stichprobe über weniger als 20 ha LF. Es wurden hier nur drei Betriebe mit einer Flächenausstattung von mehr als 50 ha LF herangezogen. Davon bewirtschaftet der größte Betrieb dieser Gruppe 75 ha LF.

\subsection{Betriebsbefragung}

Die Befragung erfolgte schriftlich anhand eines Fragebogens (siehe Anhang A.4.2). Es wurden dabei Informationen zu der Produktionsstruktur, der Faktorausstattung und der praktikablen Formen der Zusammenarbeit der landwirtschaftlichen Betriebe erfragt. Außerdem wurden hier Fragen nach den subjektiven Einschätzungen, bezüglich der Entwicklungsmöglichkeiten der überbetrieblichen Zusammenarbeit im Maschineneinsatz gestellt. Anschließend wurden die Landwirte nach weiteren Betriebsentscheidungen wie ggf. weiteren Betriebsinvestitionen befragt. Im Folgenden werden die Ergebnisse bei Verzicht auf eine explizite statistische Auswertung, in Form von allgemeinen, für das Forschungsvorhaben relevanten Aussagen dargestellt. Die detaillierten Ergebnisse der Befragung werden im Anhang zusammengestellt (siehe A. 5)

\subsubsection{Befragungsergebnisse der Region Ermland - Masuren}

Die Gruppe der untersuchten Betriebe besteht ausschließlich aus privaten Betrieben in Form von Einzelunternehmen. Wie bereits erwähnt ist der Anteil der Betriebe, die in einer anderen Rechtsform organisiert werden, in ganz Polen sehr niedrig. Diese Betriebe haben in der

\footnotetext{
${ }^{26}$ Die Vorgehensweise bei der Gestaltung dieser Stichprobe basiert auf in den bereits angeführten Aspekten der Flächenanteile an der gesamten LF der Region. Nach dem polnischen Gesetz sowie dem Landwirtschaftsentwicklungsprogramm des Landwirtschaftsministeriums werden in Polen in erster Linie die landwirtschaftlichen Familienbetriebe bis $\mathrm{zu}$ einer Flächenausstattung von 300 ha LF gefördert (vgl. Dz.U.03.64.592).
} 
Befragungsregion erst ab einer Größenordnung von 1.000-1.500 ha LF eine Bedeutung. Es handelt sich dabei zum großen Teil um Betriebe mit Beteiligung von ausländischem Kapital. Aus den bereits genannten Gründen wurden diese Betriebe nicht in die Betrachtung einbezogen.

Die Böden in den Betrieben der Stichprobe sind i.d.R. mittlerer und schwacher Qualität, d.h. die Spannbreite reicht von Klasse III bis VI. Eine relativ große Bedeutung kommt hier der Flächenpacht zu. Der Anteil der Pachtflächen an der gesamten LF der untersuchten Betriebe beläuft sich auf einem Niveau von fast $28 \%$. Dabei sind Unterschiede zwischen einzelnen Betriebsgrößengruppen offensichtlich. Während der Anteil der Pachtflächen in den Betrieben der Größengruppe 20-30 ha LF lediglich 18,4 \% beträgt, bestehen die LF der Betriebe der Größengruppe 100-300 ha LF zu ca. 44 \% aus Pachtflächen (siehe Tabelle 5-5, S. 110).

Im Hinblick auf die Art der Flächennutzung sind für die untersuchten Betriebe relativ hohe Anteile an Grünland signifikant. Dies betrifft insbesondere die Betriebe der ersten zwei Größengruppen, wo die LF zu $45 \%$ bzw. $50 \%$ aus Grünland besteht. Eine geringere Bedeutung kommt dem Grünland in den Betrieben der Größengruppen 50-100 ha LF und 100-300 ha LF zu. Bei diesen Betriebsgruppen werden 23 \% bzw. 37,2 \% der LF als Grünland bewirtschaftet.

Die Betriebe der Größengruppe von 20-50 ha LF sind im Wesentlichen Gemischtbetriebe. Bei der Tierhaltung handelt es sich vorwiegend um die Milchkuhhaltung. In zwei Betrieben werden Zuchtpferde in Beständen von 11 bzw. 12 Pferden gehalten. Darüber hinaus enthält die Gruppe einen Betrieb mit Ferkelaufzucht. Der Flächenausstattung entsprechend erfolgt die Tierhaltung in relativ kleinen Beständen. Im Durchschnitt befinden sich in einem Betrieb nur 24 VE. Ähnlichen Produktionscharakter weisen die Betriebe der Größengruppe 50-100 ha LF auf. In diese Gruppe werden neben den Milchkuhhaltungs- auch zwei Schweinehaltungsbetriebe gefasst. In beiden Fällen handelt es sich um Schweinemast. Bei dieser Gruppe entfallen auf einen Betrieb im Schnitt 29 VE. Im Rahmen der in diesen Betrieben erfolgenden Pflanzenproduktion kommt eine besondere Bedeutung dem Getreideanbau zu, allen voran dem Weizen- und Triticaleanbau.

Eine zunehmende Spezialisierung ist in den Betrieben der Größengruppe 100-300 ha LF festzustellen. Zwei Betriebe dieser Gruppe können als reine Marktfruchtbetriebe eingestuft werden. Drei weitere Betriebe können als spezialisierte Milchkuhhaltungsbetriebe mit einer ergänzenden Pflanzenproduktion bezeichnet werden. Im Vergleich zu den Betriebsgruppen 
mit geringerer Flächenausstattung sind in diesem Fall die Tierbestände wesentlich größer. Es entfallen auf einen Betrieb ca. 62 VE.

Bei dem Arbeitskräftebesatz je Flächeneinheit sind zwischen den einzelnen Betriebsgrößengruppen erhebliche Unterschiede festzustellen. Während in den Betrieben der Größengruppe 20-30 ha LF ungefähr 7,5 AK pro 100 ha LF vorliegen, ist die AK-Ausstattung in den Betrieben der Größengruppe 100-300 ha LF wesentlich niedriger und geht im Schnitt nicht über 2 AK pro 100 ha LF hinaus. Es dominieren die Familienarbeitskräfte gegenüber den familienfremden AK. Während in drei Betrieben mit einer Flächenausstattung von über 100 ha LF eine bis zwei Personen fest eingestellt werden, beschränkt sich der Einsatz der Fremdarbeitskräfte in den kleineren Betrieben i.d.R. auf gegenseitige Nachbarschaftshilfe oder Inanspruchnahme von Fremdenleistung in saisonalen Arbeitsspitzen. Im Fall der Familienarbeitskräfte handelt es sich v.a. um Personen, für die die Beschäftigung im eigenen landwirtschaftlichen Betrieb die einzige erwerbsmäßige Einkommensquelle darstellt. Lediglich in drei Fällen gehen die in einem landwirtschaftlichen Betrieb tätigen Personen einer zusätzlichen, außerlandwirtschaftlichen Tätigkeit nach.

Kennzeichnend für die untersuchten Betriebe ist eine hohe Maschinenausstattung. In vielen der Betriebe gibt es mehr als einen Schlepper (siehe Anhang A.5 Tabelle 4). Auch an dieser Stelle sind wesentliche Unterschiede zwischen den einzelnen Betriebsgrößengruppen zu verzeichnen. Einer Motorleistung der Schlepper in den Betrieben der Größengruppe 20-30 ha LF von insgesamt 527 PS pro 100 ha LF, steht eine Motorleistung von 196 PS bei den Betrieben der Größengruppe 100-300 ha LF gegenüber. Dies spiegelt sich in dem auf einen Schlepper entfallenden Flächenvolumen wider. Während einem Schlepper in den Betrieben 20-30 ha LF ca. 13 ha LF entsprechen, entfallen auf einen Schlepper in den Betrieben der Betriebsgröße 100-300 ha LF ca. 41 ha LF. Der überwiegende Teil der Schlepper besteht aus polnischen Fabrikaten. Es handelt sich dabei vornehmlich um Schlepper der Motorleistung von 30-60 PS. Außerdem sind in den untersuchten Betrieben Schlepper aus ehemaligen sozialistischen Ländern relativ stark vertreten. Es handelt sich dabei um Schlepper mit einer Motorleistung von über 60 PS, allen voran um die Marken Zetor und MTZ. 
Tabelle 5-5: Ausgewählte Ergebnisse der Befragung in Ermland - Masuren

\begin{tabular}{|c|c|c|c|c|c|}
\hline Betriebsgrößen in ha LF & $20-30$ & $30-50$ & $50-100$ & $100-300$ & über 300 \\
\hline Stichprobenumfang & 5 & 8 & 10 & 5 & 1 \\
\hline $\begin{array}{l}\text { LF in ha insgesamt } \\
\text { Anteile an LF in \% } \\
\text { - Pachtfläche } \\
\text { - Grünland } \\
\text { - Ackerland }\end{array}$ & $\begin{array}{c}142 \\
\\
13,4 \\
45,3 \\
54\end{array}$ & $\begin{array}{c}342 \\
\\
25 \\
50,3 \\
43,3\end{array}$ & $\begin{array}{c}627,1 \\
\\
33,7 \\
23 \\
64,6\end{array}$ & $\begin{array}{l}701,1 \\
43,8 \\
37,2 \\
62,2\end{array}$ & $\begin{array}{c}335 \\
\\
9 \\
0 \\
100\end{array}$ \\
\hline $\begin{array}{l}\text { Getreideanbau in ha } \\
\text { - Roggen } \\
\text { - Weizen } \\
\text { - Triticale } \\
\text { - Raps } \\
\text { - Mais }\end{array}$ & $\begin{array}{c}5 \\
32,7 \\
18,5 \\
- \\
8,8\end{array}$ & $\begin{array}{c}8 \\
51,7 \\
33,5 \\
24,3 \\
16,8\end{array}$ & $\begin{array}{c}8,2 \\
306 \\
51,3 \\
69 \\
18,5\end{array}$ & $\begin{array}{c}19 \\
243 \\
104 \\
69 \\
23\end{array}$ & $\begin{array}{c}0 \\
240 \\
0 \\
95 \\
0\end{array}$ \\
\hline ø Viehbesatz pro Betrieb in VE & 25 & 24 & 21 & 62 & 0 \\
\hline Arbeitskräfte in AK/ 100 ha LF & 7,4 & 4,73 & 3,7 & 1,95 & 2,3 \\
\hline $\begin{array}{l}\text { Motorleistung der Schlepper in PS/100 ha LF } \\
\text { Alter der Schlepper (Jahre) } \\
\text { Auslastung der Schlepper in h/Jahr }\end{array}$ & $\begin{array}{r}527 \\
20,1 \\
175\end{array}$ & $\begin{array}{c}243 \\
19 \\
228\end{array}$ & $\begin{array}{l}249 \\
19,2 \\
340\end{array}$ & $\begin{array}{l}196 \\
18,5 \\
480\end{array}$ & $\begin{array}{c}170 \\
14 \\
562\end{array}$ \\
\hline $\begin{array}{l}\text { Durchschnittsalter der Mähdrescher (Jahre) } \\
\text { Auslastung der Mähdrescher in h/Jahr } \\
\text { davon überbetrieblich }\end{array}$ & $\begin{array}{l}24 \\
70 \\
38\end{array}$ & $\begin{array}{l}23 \\
80 \\
24\end{array}$ & $\begin{array}{l}25 \\
82 \\
10\end{array}$ & $\begin{array}{c}16 \\
175 \\
70\end{array}$ & $\begin{array}{c}20 \\
190 \\
0\end{array}$ \\
\hline
\end{tabular}

Quelle: eigene Erhebung 
Charakteristisch für die eingesetzten Schlepper ist ein hohes Alter. Dabei sind die Schlepper in den Betrieben der Größengruppe 100-300 ha LF geringfügig jünger als die Schlepper in anderen Betrieben. Ungefähr $41 \%$ der Schlepper werden bereits über 20 Jahre gebraucht. Nahezu $50 \%$ der Maschinen sind der Altersgruppe von 10-20 Jahren zuzuordnen. Den restlichen Teil der Gruppe machen Schlepper aus, die weniger als 10 Jahre eingesetzt werden (6 Schlepper). Der Auslastungsgrad der eingesetzten Schlepper steigt proportional zu der Flächengröße der einzelnen Betriebe. Während ein Schlepper in den Betrieben der Größengruppe 10-30 ha LF im Schnitt 175 Stunden pro Jahr eingesetzt wird, werden die Schlepper in den Betrieben der Größengruppen 50-100 ha und 100-300 ha durchschnittlich je 340 Stunden bzw. 480 Stunden pro Jahr genutzt.

Ferner ist für die untersuchten Betriebe eine große Anzahl von Mähdreschern kennzeichnend. Ähnlich wie bei den Schleppern handelt es sich hier vornehmlich um die Mähdrescher polnischer Produktion. Nur fünf Betriebe der Stichprobe verfügen über Mähdrescher ausländischer Herkunft. In vier Betrieben sind dies gebrauchte, importierte Maschinen mit einer Motorleistung von weniger als 100 PS und einem Schneidwerk, das kleiner als 2,8 m ist. Die Mähdrescher zeichnen sich durch ein hohes Alter aus. Von den insgesamt 18 Mähdreschern weisen 12 Maschinen ein Alter von über 20 Jahren auf. Weitere fünf Mähdrescher sind der Altersgruppe von 15-20 Jahren zugeordnet. Der jüngste und gleichzeitig der einzige Mähdrescher der Altersgruppe von 1-10 Jahren ist sieben Jahre alt. Die Auslastung der Mähdrescher steigt parallel zu der Flächengröße der Betriebe an. Sie pendelt sich von ca. 73 Stunden pro Jahr in den Betrieben der Größengruppe 20-30 ha LF bis zu ca. 192 ha in den Betrieben der Größengruppe 100-300 ha LF ein.

Die Maschinenarbeiten werden in den Betrieben der Stichprobe hauptsächlich im Rahmen der Eigenmechanisierung erledigt. Bis auf eine der letzten GLZ liegen in dieser Region keine organisierten Formen der Zusammenarbeit im Bereich der Maschinennutzung vor. In drei Betrieben der Stichprobe werden Dienstleistungen der GLZ bei der Düngerausbringung und dem Mähdrusch in Anspruch genommen. Darüber hinaus sind in der Befragungsregion Zusammenschlüsse von einigen benachbarten Landwirten bei der Anschaffung von Pflanzenschutzspritzen und Düngerstreuern anzutreffen, die bei staatlicher Unterstützung vor Jahren gegründet wurden (siehe hierzu S. 87 f.). Sowohl die Einteilung der Einsatzzeit als auch die Wartungs-, Pflege und Reparaturverantwortung werden hier generell nicht im Vorfeld bestimmt. Die Maschinen werden nach jeweiligem Bedarf nach einer Absprache in den beteiligten Betrieben eingesetzt und repariert. In der Stichprobe gibt es drei Betriebe, in 
denen Pflanzenschutzspritzen in diesen Zusammenschlüssen gemeinsam eingesetzt werden. Ferner kommt hier eine relativ große Bedeutung der Nachbarschaftshilfe zu. Es handelt sich dabei vornehmlich um Grassilagearbeiten oder Einsatz der eigenen AK bei Erntearbeiten. In ähnlicher Form wird der Mähdrusch organisiert, dabei handelt es sich allerdings bis auf drei Fälle um entgeltliche Dienstleistung.

Die Eigenmechanisierung bleibt hier die bevorzugte Form der Maschinenarbeitserledigung. Dies spiegelt sich weiterhin in den Antworten auf die Frage nach den Entwicklungsmöglichkeiten verschiedener Formen der gemeinsamen Maschinennutzung wider. In keinem der untersuchten Betriebe wurde die Entwicklung von Maschinengemeinschaften als zukunftsträchtig bezeichnet. Besondere Schwierigkeiten bei dieser Form der Zusammenarbeit liegen nach den Angaben aller Landwirte im sozialen Bereich. Gemeint wird v.a. das Fehlen von Verantwortungsgefühl der Beteiligten beim Einsatz, Wartung und Reparatur der gemeinsamen Maschinen. Es ist dabei anzumerken, dass der Begriff Maschinengemeinschaft im Kreis der befragten Landwirte mit den oben genannten GLZ sowie den Zusammenschlüssen beim Einsatz von Pflanzenschutzspritzen und Düngerstreuern assoziiert wird. Die in diesen Formen der Zusammenarbeit gesammelten negativen Erfahrungen werden als ein wesentliches Hindernis bei weiterer Entwicklung der gemeinsamen Maschinennutzung aufgefasst. Die Idee des organisierten Austausches der Maschinenleistung nach dem Vorbild der deutschen MR ist in der Mehrheit der Betriebe nicht bekannt. Nach der Klärung der Grundsätze dieser Form der Zusammenarbeit wird sie von den befragten Landwirten als wenig geeignet für diese Region bezeichnet. Als Argument hierfür wird zumeist der organisatorische Aufwand angeführt. In lediglich vier Betrieben werden gute Entwicklungschancen den professionellen Lohnunternehmen eingeräumt. Genannt werden dabei Arbeiten im Bereich von Pflanzenschutz und Mähdrusch. In der Mehrheit der Antworten kommt als bevorzugte Form der überbetrieblichen Maschinennutzung die Nachbarschaftshilfe vor (siehe Tabelle 5-6).

Tabelle 5-6: Akzeptanz verschiedner Kooperationsformen in den untersuchten Betrieben in Ermland- Masuren

\begin{tabular}{|c|c|c|c|}
\hline & \multicolumn{3}{|c|}{ Anzahl der Aussagen } \\
\cline { 2 - 4 } & positiv & negativ & indifferent \\
\hline Nachbarschaftshilfe & 25 & - & 4 \\
Kleine Gemeinschaften & 0 & 25 & 4 \\
Große Gemeinschaften & 0 & 29 & 0 \\
Zusammenarbeit mit LU & 4 & 15 & 10 \\
\hline
\end{tabular}


Die Fragen nach Entwicklungsentscheidungen wurden in 19 Betrieben mit der Absicht einer weiteren Betriebsvergrößerung, sei es durch Flächenexpansion oder Bestandsaufstockung, beantwortet. Als bevorzugte Art der Flächenexpansion wird der Kauf genannt. Eine besondere Rolle spielt dabei der Sicherheitsaspekt. Darüber hinaus wird der Flächenkauf, insbesondere bei Genuss von Vorzugskrediten und im Hinblick auf die künftige Bodenpreisentwicklung, als besonders lohnende Investition betrachtet. Die verhältnismäßig geringe Bedeutung, die der Flächenpacht in den Zukunftsplänen der untersuchten Betriebe beigemessen wird, gründet sich in einem geringen Angebot von arrondierten und günstigen Pachtflächen in der Befragungsregion.

Im Fall von Maschineninvestitionen wird dem Schlepperersatz eine große Bedeutung beigemessen. In vier Betrieben wird der Schlepperkauf als eine Prioritätsinvestition bezeichnet. Dabei wird der Kauf von gebrauchten importierten Schleppern beabsichtigt. Bei der Frage nach der voraussichtlichen Finanzierung der geplanten Investitionen wird in der Mehrheit der Betriebe die Inanspruchnahme von Vorzugskrediten mit einem erforderlichen Anteil des Eigenkapitals von 20 \% erwähnt. In zwei Betrieben der Stichprobe wird, wegen der Ausschöpfung der zugänglichen Kreditlinien aus dem Förderungsprogramm, die Wahrnehmung von herkömmlichen Krediten bedacht. Lediglich in zwei Betrieben wird eine Investitionsfinanzierung durch Leasing in Betracht gezogen. Diese Finanzierungsform wird in den meisten Betrieben aufgrund ihrer großen Unsicherheit abgelehnt.

In 10 Betrieben der Stichprobe wird keine weitere Entwicklung angestrebt. Dabei handelt es sich in drei Fällen um Betriebe, die nach der Übergabe dem Nachfolger, aller Voraussicht nach, im Nebenerwerb mit der Möglichkeit der Verpachtung eines Teils der Flächen weiter bewirtschaftet werden. In zwei weiteren Betrieben wird als Argument gegen eine weitere Entwicklung eine bereits ausreichende Betriebsgröße angeführt. Die übrigen 5 Betriebe, in denen keine weitere Entwicklung beabsichtigt wird, werden wegen der Nachfolgeunsicherheit, entweder durch Verkauf oder Verpachtung, allmählich aufgelöst. In keinem der untersuchten Betriebe wird die Möglichkeit der Produktionsextensivierung und Aufnahme einer außerlandwirtschaftlichen Berufstätigkeit in Betracht gezogen.

\subsubsection{Befragungsergebnisse der Region Lublin}

In der Stichprobe der Region Lublin wurden, der dortigen Landwirtschaftsstruktur entsprechend, ähnlich wie in der ersten Region ausschließlich private Familienbetriebe erfasst. Signifikant für diese Betriebe ist ein großer Anteil von Flächen höherer Qualität. Es überwiegen hier Böden der Klassen III a, III b und IV a. Die Bedeutung der Flächenpacht ist 
von Betrieb zu Betrieb unterschiedlich und nimmt tendenziell mit der wachsenden Betriebsgröße zu. Während in den Betrieben des Größenbereichs 2-20 ha LF insgesamt knapp $10 \%$ aller Flächen aus Pachtflächen bestehen, beträgt der Anteil dieser Flächen ca. 27 \% bzw. $42 \%$ in den Betrieben der Größengruppe 20-50 ha LF und über 50 ha LF. Die vorhandenen Flächen werden nahezu ausschließlich als Ackerland bewirtschaftet. Der Anteil des dauerhaften Grünlands ist in den Betrieben der Untersuchungsprobe, unabhängig von der Betriebsgröße sehr niedrig (siehe Tabelle 5-7, S.116).

Kennzeichnend für die Betriebe ist eine hohe Produktionszersplitterung. Die Mehrzahl der Betriebe kann als Gemischtbetriebe bezeichnet werden. Dabei handelt es sich zum großen Teil um Subsistenzwirtschaften. Im Rahmen der Pflanzenproduktion kommt dem Getreideanbau vorrangige Bedeutung zu. Es werden ca. $78 \%$ aller Ackerflächen der Untersuchungsbetriebe für Getreideanbau, allen voran für Weizen- und Gersteanbau beansprucht. Die Hackfrüchte als zweitwichtigste Pflanzenart nehmen ca. $16 \%$ aller Ackerflächen in Anspruch. Die Tierhaltung erfolgt in den Untersuchungsbetrieben in kleinen Tierbeständen, die zum großen Teil der Deckung des eigenen Nahrungsbedarfs dienen.

Eine zunehmende Spezialisierung ist in den Betrieben mit einer Flächenausstattung von über 20 ha LF erkennbar. In dieser Größengruppe befinden sich zwei Betriebe mit jeweils 16 und 27 Milchkühen. In einem Betrieb dieser Größengruppe, der als reiner Tierhaltungsbetrieb bezeichnet werden kann, werden 340 Mastschweine gehalten. In weiteren vier Betrieben erfolgt die Schweinehaltung in Beständen von jeweils 20 Mastschweinen.

In den Betrieben dieser Stichprobe werden viele AK gebunden. Dabei handelt es sich nahezu ausschließlich um Familien- AK. Die Beschäftigung von fremden AK geht hier generell nicht über den Rahmen der gelegentlichen Nachbarschaftshilfe hinaus. Für ca. $37 \%$ der befragten Betriebsleiter stellt die Beschäftigung im landwirtschaftlichen Betrieb die einzige erwerbsmäßige Einkommensquelle dar. In jedem zweiten Betrieb gibt es zumindest eine Person, die hauptberuflich außerhalb des landwirtschaftlichen Betriebes beschäftigt ist. Davon handelt es sich in vier Fällen um Personen, die selbständig sind.

Die Betriebe der Stichprobe zeichnen sich durch eine hohe Maschinenausstattung aus. Je 100 ha LF entfällt eine Motorleistung der Schlepper von insgesamt 490 PS. Die zahlreichste Gruppe bilden hier relativ kleine Schlepper mit einer Motorleistung von 25 bis 55 PS (ca. $70 \%$ der Stichprobe). Es handelt sich dabei fast ausschließlich um polnische Fabrikate. Als ein weiterer Indikator der Mechanisierung dieser Betriebe kann die einem 
Schlepper gegenüberstehende Flächeneinheit in ha LF herangezogen werden. Demzufolge entfallen auf einen Schlepper ca. 12 ha LF. Die eingesetzten Maschinen sind zum großen Teil veraltet und verschlissen. Fast die Hälfte aller Schlepper der Betriebe wird bereits über 20 Jahre gebraucht. Der Anteil der Schlepper, die über 10 bis 20 Jahre genutzt werden, beträgt ca. 40 \%. Lediglich 5 Schlepper werden weniger als 5 Jahre eingesetzt. Der Flächengrößen der untersuchten Betriebe entsprechend niedrig ist der Auslastungsgrad der eingesetzten Schlepper. Lediglich in 5 Betrieben reicht die jährliche Leistung eines Schleppers über 300 Stunden hinaus. Signifikant für die Stichprobe ist eine relativ große Anzahl von Mähdreschern. In 12 Betrieben sind eigene Mähdrescher vorhanden. Es handelt sich dabei nahezu ausschließlich um polnische Fabrikate mit einer Motorleistung von 120 PS und einem Schneidwerk von 4,2 m. Die Mähdrescher zeichnen sich ähnlich wie die Schlepper durch ein hohes Alter aus. Die Mehrheit der Mähdrescher wird bereits über zwanzig Jahre genutzt. Der jährliche Auslastungsgrad beläuft sich auf sehr niedrigem Niveau und geht nur in einem Fall über 100 Stunden pro Jahr hinaus.

Bekannt aus der frühen Vergangenheit sind in dieser Region, wie in Ermland - Masuren, Zusammenschlüsse von einigen Landwirten zur Anschaffung und anschließenden gemeinsamen Nutzung von Pflanzenschutzspritzen. Es wird zzt. in keinem der untersuchten Betriebe diese Form der Zusammenarbeit praktiziert. Des Weiteren funktioniert in der Untersuchungsregion eine GLZ. Das Angebot dieser Organisation, das sich auf den Einsatz von Schleppern, Mähdreschern, Anhängern und Grasmähern erstreckt, wird jedoch von keinem der untersuchten Betriebe in Anspruch genommen. Ferner werden von drei Betrieben ein Sägerät, ein Schlepper und ein Düngerstreuer gemeinsam benutzt. Die Maschinen bilden kein gemeinsames Eigentum. Sie wurden separat angeschafft und werden nach Absprache auf Basis gegenseitigen Leistungsaustausches eingesetzt. In all diesen Fällen handelt es sich um einen Verwandtenkreis. Weiterhin hat in dieser Region auch die Nachbarschaftshilfe eine wesentliche Bedeutung. Dabei handelt es sich um den Einsatz von Mähdreschern und Rübenbunkerköpfrodern, der nicht auf Basis von gegenseitiger Dienstleistung, sondern vielmehr im Rahmen von entgeltlicher Lohnmaschinenarbeit erfolgt. 
Tabelle 5-7: Ausgewählte Ergebnisse der Befragung in Lublin

\begin{tabular}{|c|c|c|c|c|c|}
\hline Betriebsgrößen in ha LF & $2-5$ & $5-10$ & $10-20$ & $20-50$ & über 50 \\
\hline Stichprobenumfang & 3 & 7 & 7 & 7 & 3 \\
\hline $\begin{array}{l}\text { LF in ha insgesamt } \\
\text { Anteile an LF in \% } \\
\text { - Pachtfläche } \\
\text { - Grünland } \\
\text { - Ackerland }\end{array}$ & $\begin{array}{c}13,6 \\
0 \\
0 \\
100\end{array}$ & $\begin{array}{c}45,5 \\
\\
17 \\
0 \\
100\end{array}$ & $\begin{array}{l}110 \\
11 \\
1,8 \\
98\end{array}$ & $\begin{array}{c}206,5 \\
20 \\
0 \\
100\end{array}$ & $\begin{array}{c}189 \\
\\
35 \\
0 \\
100\end{array}$ \\
\hline $\begin{array}{l}\text { Getreideanbau in ha } \\
\text { - Roggen } \\
\text { - Weizen } \\
\text { - Gerste } \\
\text { - Triticale } \\
\text { - Raps } \\
\text { - Mais }\end{array}$ & $\begin{array}{c}0 \\
7 \\
4,0 \\
0 \\
2,5 \\
0\end{array}$ & $\begin{array}{c}0 \\
17,5 \\
9,5 \\
0 \\
5,5 \\
0\end{array}$ & $\begin{array}{c}0 \\
35,5 \\
23,8 \\
0 \\
6 \\
6\end{array}$ & $\begin{array}{c}6 \\
70,5 \\
45 \\
16 \\
16 \\
13\end{array}$ & $\begin{array}{c}0 \\
63 \\
35 \\
18,5 \\
25,5 \\
14\end{array}$ \\
\hline $\begin{array}{l}\text { Hackfrüchte in ha } \\
\text { - Zuckerrüben }\end{array}$ & - & 10 & 15,5 & 30,5 & 31 \\
\hline$\varnothing$ Viehbesatz pro Betrieb in VE & 3,5 & 20 & 6 & 15 & 27 \\
\hline Arbeitskräfte in AK/ 100 ha LF & 28 & 13,4 & 10,5 & 6,7 & 3,3 \\
\hline $\begin{array}{l}\text { Motorleistung der Schlepper in PS/100 PS } \\
\text { Alter der Schlepper (Jahre) } \\
\text { Auslastung der Schlepper in h/Jahr }\end{array}$ & $\begin{array}{c}1020 \\
31 \\
220\end{array}$ & $\begin{array}{c}494 \\
24 \\
290\end{array}$ & $\begin{array}{c}580 \\
25 \\
260\end{array}$ & $\begin{array}{c}560 \\
21 \\
260\end{array}$ & $\begin{array}{c}390 \\
12 \\
300\end{array}$ \\
\hline $\begin{array}{l}\text { Durchschnittsalter der Mähdrescher (Jahre) } \\
\text { Auslastung der Mähdrescher in h/Jahr } \\
\text { davon überbetrieblich }\end{array}$ & $\begin{array}{l}20 \\
32 \\
25\end{array}$ & $\begin{array}{c}25 \\
15 \\
0\end{array}$ & $\begin{array}{l}19 \\
70 \\
27\end{array}$ & $\begin{array}{l}18 \\
66 \\
20\end{array}$ & $\begin{array}{c}19 \\
115 \\
25\end{array}$ \\
\hline
\end{tabular}

Quelle: eigene Erhebung 
In den Betrieben der Stichprobe wird eine weitere Eigenmechanisierung angestrebt. Als einzige praktikable Form überbetrieblicher Maschinennutzung wird Nachbarschaftshilfe angesehen. In keinem der untersuchten Betriebe werden Entwicklungschancen einer Maschinengemeinschaft oder einem Lohunternehmen beigemessen. Als Gegenargumente für Maschinengemeinschaften werden soziale Aspekte der Zusammenarbeit angeführt. Die Auslagerung der Maschinenarbeiten an Lohnunternehmen wird v.a. aus finanziellen Gründen als undurchführbar bezeichnet. Diese Form der Mechanisierung wird von den befragten Landwirten als zu teuer bezeichnet. Ähnlich wie in der nördlichen Region wird hier die Idee der Zusammenarbeit beim Maschinenaustausch im Rahmen von MR in meisten Fällen als unrealistisch beurteilt (siehe Tabelle 5-8).

Tabelle 5-8: Akzeptanz verschiedner Kooperationsformen in den untersuchten Betrieben in Lublin

\begin{tabular}{|c|c|c|c|}
\hline & \multicolumn{3}{|c|}{ Anzahl der Aussagen } \\
\cline { 2 - 4 } & positiv & negativ & 5 \\
Nachbarschaftshilfe & 22 & - & 3 \\
Kleine Gemeinschaften & 1 & 24 & 0 \\
Große Gemeinschaften & 0 & 27 & 3 \\
Zusammenarbeit mit LU & 0 & 24 & \\
\hline
\end{tabular}

Eine weitere Betriebsentwicklung, sei es in Form von Bestandsaufstockung oder Flächenzukauf, wird von 14 der befragten Landwirte angestrebt. Bei Flächenexpansion wird der Pacht eine vorrangige Bedeutung beigemessen. Als Grund hierfür wird v.a. der hohe Bodenpreis in dieser Region genannt. Im Fall von Maschineninvestitionen wird eine große Bedeutung der Erneuerung des vorhandenen Maschinenparks beigemessen. Es handelt sich dabei vornehmlich um Schlepperersatz und Anschaffung von modernen Geräten zur Bodenbearbeitung. In zwei Betrieben wird eine Anschaffung eines Mähdreschers bedacht. Die geplanten Investitionen sollen vorrangig durch konventionelle Kredite finanziert werden. Ebenso selten wie in der nördlichen Untersuchungsregion wird hier die Finanzierung durch Leasing in Betracht gezogen.

In der Gruppe der weiteren 13 Betriebe in denen kein Wachstum angestrebt wird, befinden sich 7 Betriebe, die weiterhin im Nebenerwerb bewirtschaftet werden. Die restlichen 6 Betriebe werden aus Altersgründen des Betriebsleiters und wegen unsicherer Nachfolge durch Flächenverkauf oder -Verpachtung allmählich aufgelöst. 


\subsection{Zusammenfassung}

Die Betrachtungen dieses Kapitels veranschaulichen deutliche Unterschiede in der Landwirtschaftsstruktur beider Untersuchungsregionen. Während es in der nördlichen Region im Jahr 2002 zusammengerechnet ca. 52.000 landwirtschaftliche Betriebe gab, ging die Anzahl der Betriebe in der Region Lublin über 223.000 hinaus. Dabei ist der Umfang der gesamten LF in der südlichen Region lediglich um knapp ein Viertel größer als in der Woiwodschaft Ermland - Masuren. Für die Woiwodschaft Ermland - Masuren ist ein hoher Anteil von größeren Betrieben signifikant. In der Bewirtschaftung der Betriebe mit einer Flächenausstattung von über 100 ha $\mathrm{LF}$ befinden sich ca. $40 \%$ aller LF. Das Erscheinungsbild der Landwirtschaft in der Region Lublin prägen demgegenüber kleine und sehr kleine Betriebe. Allein auf die Betriebe des Größenbereichs 1-20 ha LF entfallen ca. $80 \%$ aller LF dieser Region.

Die dargestellten Ergebnisse erlauben, Tendenzaussagen über die Struktur und die daraus resultierenden allgemeinen Entwicklungsmöglichkeiten der Landwirtschaft $\mathrm{zu}$ treffen. In Ermland-Masuren kann von günstigen Entwicklungsbedingungen für größere spezialisierte Einheiten gesprochen werden. Dabei handelt es sich um Betriebe des Veredlungsbereichs und des Marktfruchtanbaus. Eine wesentliche Bedeutung in der Bewirtschaftung der Betriebe dieser Region kommt der Flächenpacht zu. Daraus kann allerdings nur bedingt der Schluss abgeleitet werden, dass sich der Strukturwandel in puncto Flächenbesitz auf dem Wege der Pacht vollzieht. Dies gründet sich darin, dass ein hoher Anteil der Pachtflächen dieser Region auf die immer noch nicht privatisierten LF der ANR entfällt. Für die Region Lublin ist eine starke Produktionszersplitterung signifikant. Der Anteil von Betrieben, die als spezialisierte Betriebe bezeichnet werden, ist hier außerordentlich niedrig. Die meisten LF in dieser Region werden von ihren Eigentümern bewirtschaftet. Die Bedeutung von Flächenpacht nimmt zwar in den größeren Betrieben zu, ist aber insgesamt sehr niedrig. Der Wandel zu größeren existenzsicheren Einheiten wird hier im Vergleich zu der nördlichen Region wesentlich langsamer verlaufen. Eine Chance besonders für die kleineren Betriebe dieses Gebiets kann in der Entwicklung von alternativen arbeitsintensiveren Produktionszweigen bestehen.

Die Untersuchungsbetriebe beider Regionen weisen bestimmte strukturelle Ähnlichkeiten auf. Dies betrifft zunächst die AK- Ausstattung. Von einzelnen Fällen abgesehen, sind in den Betrieben der Stichprobe überproportional viele AK gebunden. Dies schlägt sich auch in der Arbeitsproduktivität nieder. Ferner charakteristisch für beide Regionen ist eine rückständige 
technische Ausstattung. Die vorhandenen Maschinen zeichnen sich durch ein hohes Alter und einen großen Abnutzungsgrad aus. Die vorhandenen Maschinen werden wenig ausgelastet. Die Möglichkeiten der Vornahme von Ersatzinvestitionen sind durch die Kapitalknappheit eingeschränkt. Die überbetriebliche Zusammenarbeit hat in den Betrieben beider Stichproben eine marginale Bedeutung und geht generell nicht über den Rahmen der informellen Nachbarschaftshilfe hinaus. Die Verbreitung der Idee der Kooperation ist stark durch die aus den früheren Erfahrungen resultierende Abneigung der Landwirte gegenüber jeglichen Formen der Zusammenarbeit erschwert. Lediglich die Nachbarschaftshilfe wird als Kooperationsform zzt. akzeptiert. Schließlich sind für die ländliche Bevölkerung in beiden Regionen kaum Möglichkeiten für die Aufnahme einer erwerbsmäßigen außerlandwirtschaftlichen Tätigkeit vorhanden. 


\section{Potenzielle Mechanisierungspfade für die Untersuchungsregionen}

Es ist Ziel dieses Kapitels, anhand von Kostenkalkulationen ausgewählter Maschinen exemplarische Mechanisierungsszenarien für beide Untersuchungsregionen herauszuarbeiten. Um dieser Aufgabe hinreichend Rechnung zu tragen, werden zunächst die Kosten des Einsatzes der zzt. vorhandenen Maschinen und die Bedeutung moderner Mechanisierung für die weitere Betriebsentwicklung erörtert. Darauf aufbauend wird unter Berücksichtigung des Kooperationspotenzials und des Modernisierungsbedarfs der untersuchten Betriebe die Auswahl geeigneter Kooperationsformen diskutiert. In einem weiteren Schritt werden nach einer kurzen Schilderung der theoretischen Kalkulationsgrundlagen und der daraus resultierenden Kalkulationsmethodik exemplarische Kostenkalkulationen ausgewählter Maschinen vorgestellt. Es wird dabei neben der Anschaffung und anschließendem Einsatz neuer Maschinen auch die Variante der Auswahl gebrauchter Maschinen analysiert. Um den Praxisbezug der Arbeitsergebnisse zu sichern wurden die herausgearbeiteten Vorschläge den Beratern und anderen betroffenen Experten vor Ort zu einer kritischen Beurteilung vorgelegt. Die diesbezüglichen Erkenntnisse werden in diesem Kapitel abschließend in Form einer Diskussion dargestellt.

\subsection{Kosten des Einsatzes der vorhandenen Maschinen und die Bedeutung moderner Mechanisierung für die Betriebsentwicklung}

Um die Vorteile der Modernisierung zahlenmäßig darzustellen, müssten zunächst die Kosten des Maschineneinsatzes bei der vorliegenden Mechanisierungsstruktur erfasst und anschließend mit den Kosten der künftigen Investitionen verglichen werden. Eine genaue Abbildung der gegenwärtigen Maschinenarbeitserledigungskosten stellt im Fall der Untersuchungsprobe ein wesentliches Problem dar. Hierfür gibt es mehrere Gründe.

Wie vorher geschildert, ist für die Maschinen der Untersuchungsbetriebe ein hohes Alter kennzeichnend. Bei Unterstellung einer Abschreibungsdauer von 15 Jahren, sind ca. $78 \%$ aller Schlepper und ca. 89 \% aller Mähdrescher der Stichprobe in der Region Olsztyn sowie ca. $77 \%$ aller Schlepper und $75 \%$ aller Mähdrescher der Stichprobe in der Region Lublin abgeschrieben worden. Die festen Kosten der Maschinen sind unter diesen Bedingungen niedrig. Hinzu kommt, dass die Mehrheit der Maschinen bedingt durch einen hohen Abnutzungsgrad keinen großen Restwert hat. Daher ist die Bedeutung der erzielbaren 
Veräußerungserlöse bei der Kapitalbeschaffung für die Ersatzinvestitionen in den untersuchten Betrieben eher marginal.

Die Reparaturen werden in den untersuchten Betrieben hauptsächlich eigenständig, ohne Inanspruchnahme von Dienstleistungen Dritter erledigt. Als Auszahlungen kommen hier im Wesentlichen die Kosten der Ersatzteile vor. Da der eigenen Arbeit bei Reparaturen i.d.R. kaum Opportunitätskosten gegenüberstehen, kann hier kein hoher Lohnansatz unterstellt werden. Die aktuellen Reparaturkosten können anhand der Befragungsergebnisse in den Kalkulationen dargelegt werden. Ein weiterer Verlauf dieser Kosten kann allgemein prognostiziert werden. Es ist dabei jedoch zu beachten, dass der gesamte Reparaturaufwand relativ niedrig ist. Mithin fallen die Reparaturkosten, als einer der eventuellen Bestimmungsfaktoren für die Ersatzinvestitionen kaum ins Gewicht.

Einen Überblick über die Struktur der Schlepper- und Mähdrescherkosten in den untersuchten Betrieben beider Regionen gibt die Tabelle 6-1.

Tabelle 6-1: Anzahl der Betriebe nach der Höhe der Einsatzkosten der Schlepper und Mähdrescher in beiden Untersuchungsregionen

\begin{tabular}{|c|c|c|c|c|}
\hline Schlepperkosten ZL/h & $22-32$ & $>32-55$ & $>55-77$ & $>77$ \\
\hline $\begin{array}{c}\text { Anzahl der Betriebe in } \\
\text { Ermland - Masuren }\end{array}$ & 5 & 9 & 6 & 10 \\
\hline $\begin{array}{c}\text { Anzahl der Betriebe in } \\
\text { Lublin }\end{array}$ & 8 & 4 & 5 & - \\
\hline \hline Mähdrescherkosten ZL/h & $90-140$ & $>140-175$ & 4 \\
\hline $\begin{array}{c}\text { Anzahl der Betriebe in } \\
\text { Ermland - Masuren }\end{array}$ & 10 & 8 & & \multicolumn{2}{|c|}{} \\
\hline $\begin{array}{c}\text { Anzahl der Betriebe in } \\
\text { Lublin }\end{array}$ & 3 & 5 & & \\
\hline
\end{tabular}

Eine Gegenüberstellung der gegenwärtigen Mechanisierungskosten und der Kosten des Einsatzes neuer Maschinen kann $\mathrm{zu}$ falschen Schlussfolgerungen verleiten. Bei ausschließlicher Untersuchung der Kostenstruktur können die Maschinenarbeiten in vielen Fällen kaum günstiger erledigt werden, als dies derzeit geschieht. Die Einführung moderner Technik wird in vielen Betrieben eine Kostensteigerung zur Folge haben. Vor diesem Hintergrund werden die Mechanisierungskosten bei gegenwärtiger Mechanisierungsstruktur mit den Kosten des Einsatzes neuerer Maschinen nicht verglichen. Der Übersichtlichkeit halber werden die Kosten der zzt. in den analysierten Betrieben befindlichen Maschinen tabellarisch im Anhang komprimiert zusammengestellt (siehe Anhang A. 5) ${ }^{27}$.

\footnotetext{
${ }^{27} \mathrm{Zu}$ der Berechnungsmethodik der Kosten des Einsatzes der bisher in den untersuchten Betrieben vorhandenen Maschinen siehe S. $135 \mathrm{f}$.
} 
Der Kostenvergleich ist allein kein ausreichendes Kriterium für die Entscheidung über die künftige Mechanisierung. Es müssen an dieser Stelle auch andere Faktoren berücksichtigt werden. Dabei handelt es sich zunächst um die Effizienz des Einsatzes einzelner Produktionsfaktoren. Während diese Aspekte bei Auslaufbetrieben, deren Besitzer die landwirtschaftliche Produktion z.B. wegen des Erreichen des Renteneintrittalters bald einzustellen beabsichtigen, kaum ins Gewicht fallen, sind sie für alle Betriebe, in denen die Beschäftigung weiterhin als Haupteinkommensquelle betrachtet wird, von vorrangiger Bedeutung. In diesem Fall ist ein weiteres Betriebswachstum unausweichlich. Dieser Prozess wird zum großen Teil vom Tempo der Modernisierung der Mechanisierung determiniert. Denn die Verbesserung der Mechanisierungsstruktur steht in einem vielfältigen Zusammenhang mit der Betriebsentwicklung.

Die Einführung moderner schlagkräftiger Technik kann zur Verbesserung der Betriebsorganisation beitragen. Es handelt sich dabei im Wesentlichen um die Freisetzung von Arbeitskapazitäten für die Entwicklung in Bereichen, die im Vergleich zur bisherigen Produktionsstruktur auf eine höhere Rentabilität rechnen lassen. Hierzu zählt z.B. die innerbetriebliche Entwicklung mit Konzentration im Veredlungsbereich oder der Aufbau der Produktion mit arbeitsintensiveren Verfahren. In bestimmten Betrieben kann die Einführung moderner Technik als Ausgangspunkt zur Entwicklung professioneller Dienstleistungen betrachtet werden. Dies trifft besonders bei der Annahme zu, dass ein Teil der kleineren Betriebe beider Regionen künftig im Nebenerwerb bei Inanspruchnahme der Maschinendienstleistungen bewirtschaftet wird.

Nicht ohne Bedeutung ist der Einfluss der modernen Technik auf die Ertragsleistung. Die Steigerung der Naturalerträge, die als Voraussetzung für die Verbesserung der Einkommenssituation der Landwirte und ihrer Familienangehörigen gilt, ist im Wesentlichen von der Anwendung moderner präziser Produktionsverfahren abhängig. Dies kann wiederum ohne neue schlagkräftige Technik nicht verwirklicht werden. Von Bedeutung ist hier die Vermeidung von Ernteverlusten sowie die Termingerechtigkeit, die bei größeren Flächen den Einsatz von zuverlässigen und schlagkräftigen Maschinen voraussetzt. Bei der Minderung des Verspätungsrisikos bei bestimmten Arbeiten kann der Produktionsverlauf besser organisiert werden. Dies kann wiederum einen positiven Einfluss auf die Organisation der Vermarktung der Erzeugnisse haben. Hinzu kommt auch der Aspekt der Qualität der Erzeugnisse. Moderne Technik ist eine Voraussetzung für die Herstellung von qualitativ hochwertigen Produkten, die den steigenden Marktanforderungen entsprechen. 
Des Weiteren geht die Erfüllung der Umweltauflagen mit der Einführung moderner Technik einher. Dabei handelt es sich z.B. um die Reduktion der Emission von Abgasen oder die Einschränkung der Verluste beim Einsatz von Dünge- und Pflanzenschutzmitteln. Nicht zuletzt kann die moderne Technik mit dem Einsatz von GPS-Systemen eine Linderung für das Problem der Flurzersplitterung bieten. Mit einer elektronischen Flächenkartierung und einer präzisen Ermittlung der Erträge kann künftig die Flächenstruktur in Zusammenarbeit mehrerer Landwirte wesentlich verbessert werden, ohne dabei den mit der Änderung der Eigentumsverhältnisse verbundenen hohen Aufwand hervorzurufen.

\subsection{Geeignete Kooperationsformen}

An dieser Stelle werden zusammenfassend die Entwicklungschancen bestimmter Kooperationsformen in beiden Regionen analysiert. Dies wird im Lichte des Kapitalaufwands, der Konstellation der Produktionsfaktoren, der voraussichtlichen Betriebsentwicklung und der Akzeptanz für bestimmte Kooperationsformen erfolgen.

\section{Ermland - Masuren}

Die Befragungsergebnisse veranschaulichen, dass eine zentrale Bedeutung in der überbetrieblichen Zusammenarbeit der landwirtschaftlichen Betriebe der Region Ermland - Masuren der Nachbarschaftshilfe zukommt. Diese Form der Zusammenarbeit kann in ihrer traditionellen Ausprägung noch über längere Zeit eine vorrangige Rolle in der Mechanisierung der kleineren Betriebe spielen. Ohne gravierende Änderungen in der Konstellation der Produktionsfaktoren hervorzurufen, kann die Mehrheit der Maschinenarbeiten bei Anwendung der bereits vorhandenen Technik bei gegenseitiger Unterstützung günstig erledigt werden. Diese Form der Zusammenarbeit findet zzt. in den kleineren Betrieben auch die höchste Akzeptanz. Mit einem weiteren Wandel in den Betriebsgrößenstrukturen und einer damit einhergehenden Spezialisierung der bestehenden Betriebe wird die Nachbarschaftshilfe allmählich an Bedeutung verlieren. Es können hier neben der Eigenmechanisierung die Formen der Zusammenarbeit in den Vordergrund rücken, bei denen die steigenden Anforderungen an die Leistungsfähigkeit, die Planungssicherheit und die Arbeitsqualität leichter zu erfüllen sind. Die zwischen bestimmten Betrieben bereits bestehende lose Zusammenarbeit kann als Ausgangspunkt für die Entwicklung neuer Mechanisierungsformen, von Maschinengemeinschaften über Lohnmaschineneinsatz bis hin zur Übergabe kompletter Betriebsbewirtschaftung an Dritte, betrachtet werden. 
Der Übergang von Nachbarschafshilfe zu anderen Formen der überbetrieblichen Zusammenarbeit kann durch Aktivierung des bereits vorhandenen Potenzials von Angebot und Nachfrage der Maschinenleistung beschleunigt werden. Daher ist eine nach dem Vorbild der deutschen MR organisierte Maschinenarbeitsvermittlung für diese Region grundsätzlich zu begrüßen. Die Gründung einer gesonderten Institution mit dem Ziel, den überbetrieblichen Austausch der Maschinenleistung zu erleichtern, kann jedoch sachlich nicht gerechtfertigt werden. Ausgehend von guten Wachstumsmöglichkeiten der landwirtschaftlichen Betriebe dieser Region und der Entwicklung anderer Formen der Zusammenarbeit, könnte in den Maschinenringen schon bald das Gleichgewicht des Angebots und der Nachfrage der Maschinenleistung nicht mehr erhalten bleiben. Benötigt sind hier Maßnahmen, die die Modernisierung ohne den großen Aufwand, den der Aufbau einer neuen Organisation erfordert, zu verwirklichen helfen. In diesem Zusammenhang kommt der landwirtschaftlichen Beratung vor Ort eine zentrale Bedeutung zu. Sie hat den Modernisierungsprozess aktiv zu begleiten und entsprechend zu lenken. Neben dem direkten Zugehen auf die einzelnen landwirtschaftlichen Betriebe und damit Aufzeigen konkreter Vorteile der überbetrieblichen Mechanisierung ist hier die Beratung in Form von Fachveranstaltungen und Publikationen in Zeitschriften von wesentlicher Bedeutung.

Weiterhin liegen in dieser Region günstige Bedingungen für die Entwicklung von Maschinengemeinschaften von zwei oder drei Landwirten vor. Dies betrifft insbesondere die mittleren und größeren Betriebe. Diese Betriebe haben einen großen Wachstums- und Kapitalbildungsbedarf. Der gemeinsame Maschineneinsatz im Rahmen einer Maschinengemeinschaft kommt diesem Sachverhalt entgegen. Es handelt sich dabei im Wesentlichen um die Minderung des Kapitalbedarfs für die erforderlichen Maschinenersatzinvestitionen und um die Reduktion der Produktionskosten durch die Steigerung der Maschinenauslastung. Von Bedeutung ist hier auch die Freisetzung von Kapazitäten, sei es in Form eigener Arbeit oder finanzieller Mittel, die für eine weitere Betriebsentwicklung angewendet werden können. In der Region Ermland - Masuren bietet sich für die Gemeinschaften, in denen die Maschinenkapazitäten in den Mitgliedsbetrieben nicht hinreichend ausgelastet werden, die Möglichkeit an, als Leistungsanbieter aufzutreten. An dieser Stelle ist allerdings $\mathrm{zu}$ bemerken, dass die Maschinengemeinschaften als eine Alternative zur Eigenmechanisierung nicht für jeden Betrieb in Betracht kommen. Denn diese Form der Zusammenarbeit stellt an die Beteiligten besondere Anforderungen sozialer Natur. 
Wenig geeignet erscheinen für diese Region die Maschinengemeinschaften mit größerer Anzahl der Beteiligten. In dieser Kooperationsform sind die Mechanisierungskosten zwar besonders niedrig, eine erfolgreiche Zusammenarbeit mehrerer Landwirte in einer solchen Maschinengemeinschaft ist jedoch unter den Bedingungen der untersuchten Betriebe eher unrealistisch. Die Ursache hierfür liegt v.a. im Fehlen der Kooperationsbereitschaft, des gegenseitigen Vertrauens oder des Verantwortungsgefühls. Einer Maschinengemeinschaft mit mehreren Beteiligten werden von den befragten Landwirten, nicht zuletzt wegen der negativen Erfahrungen mit den ehemaligen GLZ, kaum Entwicklungschancen eingeräumt (siehe hierzu auch S. 87 f.).

Eine wesentliche Rolle in der weiteren Entwicklung der Mechanisierung in dieser Region kann der Lohnmaschineneinsatz spielen. Zu den Arbeitsgängen in denen in vielen Betrieben bereits jetzt die Inanspruchnahme der Lohnmaschinenleistung interessant sein kann, zählen z.B. der Mähdrusch oder der Pflanzenschutz. Die Funktion der Leistungsanbieter können in erster Linie größere Betriebe mit freien Maschinenkapazitäten sowie die oben angesprochenen kleinen Maschinengemeinschaften wahrnehmen. Für die Leistungsanbieter bietet sich dabei die Möglichkeit, die Auslastung der vorhandenen Maschinenkapazitäten zu steigern und u.U. ein zusätzliches Einkommen zu erwirtschaften. Bei einem relativ geringeren Aufwand kann die Maschinenleistung durch diese Gruppe der Betriebe zu günstigen Preisen angeboten werden. Für den Auftraggeber ergibt sich daraus der Vorteil, dass die Maschinenarbeiten in seinem Betrieb zu einem günstigen Preis erledigt werden können.

In diesem Zusammenhang kann sich hier künftig auch der Lohnmaschineneinsatz von professionellen Dienstleistungsanbietern der Landtechnik entwickeln. Als Beispiel hierzu dient die Organisation einer Silierkette mit dem Einsatz eines größeren Häckslers. Die Möglichkeit der Auslagerung der Arbeiten bei Grassilagebereitung kann besondere Bedeutung für spezialisierte Milchkuhhaltungsbetriebe haben. Dabei handelt es sich neben der Leistungssteigerung auch um die Freisetzung von Kapazitäten für eine weitere Betriebsentwicklung. $\mathrm{Zu}$ weiteren Arbeitsgängen, bei denen der professionelle Lohnmaschineneinsatz eine wesentliche Bedeutung haben kann, zählen der Mähdrusch und der Pflanzenschutz. Dabei ist neben der Leistungsfähigkeit auch die Qualität der geleisteten Arbeit wichtig. Das zweite Argument trifft besonders beim Pflanzenschutz zu, wo die Arbeitspräzision nicht nur im Hinblick auf die Naturalerträge, sondern auch wegen der steigenden Umweltauflagen an Bedeutung gewinnen wird. 


\section{Lublin}

Ähnlich wie in der nördlichen Region spielt gegenwärtig auch in Lublin die Nachbarschaftshilfe eine vorrangige Rolle in dem überbetrieblichen Maschineneinsatz. Von der Persistenz der aktuellen Betriebsgrößenstruktur in diesem Untersuchungsgebiet ausgehend, kann dieser Form der Zusammenarbeit in ihrer ursprünglichen Ausprägung noch über längere Zeit eine wesentliche Bedeutung in der Maschinenarbeitserledigung zukommen. Dies trifft besonders auf die Produktionsverfahren $\mathrm{zu}$, in denen keine Spezialmaschinen benötigt werden und solche Aspekte wie Termingerechtheit oder Leistungsfähigkeit eine untergeordnete Rolle spielen. Eine allmähliche Steigerung der Anforderungen an die Mechanisierung legt die Entwicklung der gegenseitigen Naschbarschaftshilfe zu einer entgeltlichen Dienstleistung nahe. Der Innovationseffekt der informellen Nachbarschaftshilfe, gemessen an dem Hauptkriterium der Modernisierung der technischen Ausstattung, kann insgesamt als eingeschränkt bezeichnet werden. Ein deutlicher Effekt könnte nur bei Entwicklung einer dauerhaften Zusammenarbeit zwischen den Auftragnehmern und -gebern unter der Voraussetzung einer gegenseitigen Anpassung der anstehenden Maschinenersatzinvestitionen erzielt werden. Unter Bedingungen, in denen die Nachbarschaftshilfe zwar auf monetärer Basis erfolgt, jedoch nicht über den Rahmen einer sporadischen Dienstleistung hinausgeht und somit keine nennenswerte Wirkung auf das Investitionsverhalten ausübt, könnte der Modernisierungseffekt höchstens bis zu den in den dienstleistenden Betrieben aus eigener Kraft vorgenommenen Investitionen erzielt werden.

Ähnlich wie in der nördlichen Region kann hier ein gewisser Modernisierungseffekt durch die Mobilisierung und den Austausch der bereits vorhandenen Maschinenkapazitäten angestrebt werden. Diese Entwicklung soll von außen gefördert werden. Wenig geeignet für die Verwirklichung dieser Aufgabe erscheint allerdings die Gründung von MR. Dabei treffen grundsätzlich dieselben Argumente zu, wie im Fall der nördlichen Region. Darüber hinaus wäre in dieser Region die Tätigkeit der MR aufgrund einer weitgehend homogenen Produktionsstruktur und einer vergleichbaren technischen Ausstattung der landwirtschaftlichen Betriebe wesentlich erschwert. Die MR müssten hier weit überregional aktiv werden. Dies würde den Aufwand bei der Entwicklung der Organisation steigern und zugleich die Chancen ihrer effizienten Wirkung verkleinern.

In vielen Betrieben dieser Region sind günstige Bedingungen für die Entwicklung von Maschinengemeinschaften mit zwei oder drei Mitgliedern vorhanden. Dies betrifft in erster Linie die mittleren und größeren Betriebe. Neben dem Argument der Minderung des 
Kapitalbedarfs für die Ersatzinvestitionen hat in diesem Fall auch der Aspekt der Auslastung der Maschinenkapazitäten eine wesentliche Bedeutung. Denn auch in vielen größeren Betrieben dieser Region kann die Sicherung der erforderlichen Auslastung moderner schlagkräftiger Maschinen erhebliche Probleme bereiten. Durch kooperative Maschinennutzung können diese Probleme entschärft werden. Durch Zusammenarbeit in Maschinengemeinschaften kann in Betrieben dieser Region ein schnellerer Maschinenwechsel und damit die Teilnahme am technischen Fortschritt erleichtert werden.

In vielen kleinen Betrieben dieser Region können als eine Alternative zur Eigenmechanisierung, insbesondere bei Verfahren in denen große Aggregate benötigt werden, die Maschinengemeinschaften von mehreren Beteiligten eine große Bedeutung haben. Dabei handelt sich um alle Arbeiten in denen gemeinsame Ziele verfolgt werden können. Als Beispiel dient hier die Organisation der Zuckerrübenernte und des -transports, die Getreideernte- und Transport oder auch die Gülleausbringung. Die Entwicklung einer solchen Gemeinschaft aus der Eigeninitiative und bei gemeinsamer Führung mehrerer Landwirte scheint zzt. nicht realistisch zu sein. Es handelt sich hier, ähnlich wie in der Region Ermland Masuren, v.a. um die Barrieren der sozialen Natur. Dennoch denkbar ist für diese Region eine Maschinengemeinschaft, die nach dem Vorbild der in Westdeutschland organisierten Maschinengemeinschaften zwar viele Landwirte vereinigt, tatsächlich aber nur von einer Person oder einer außen stehenden Institution geführt wird. Auf diesem Wege könnten die oben genannten Hindernisse erfolgreich gemindert werden. Ein Anstoß, der für diese Entwicklung erforderlich ist, kann von der Seite der örtlichen Beratung kommen. Hierzu nötig ist auch die Teilnahme von Pionierlandwirten, die in ihrem Umfeld eine Führungsrolle bei solchen Initiativen übernehmen. Günstige Rahmenbedingungen für größere Gemeinschaften können z.B. in Erzeugergruppen geschaffen werden.

In dieser Region kann sich künftig auch der Lohnmaschineneinsatz weiterentwickeln. Diese Entwicklung kann allerdings im Vergleich zu Ermland-Masuren einen wesentlich langsameren Verlauf haben. Die Ursache hierfür liegt v.a. darin, dass die Landwirtschaft dieses Untersuchungsgebiets $\mathrm{zu}$ kleinstrukuriert ist, als dass der Einsatz moderner schlagkräftiger Technik wirtschaftlich zum Tragen kommen könnte. Die Dienstleistung eines professionellen Lohnunternehmens könnte hier unter Berücksichtigung der durch die Flächenzersplitterung verursachten häufigen Rüstzeiten und Leerfahrten nur ab einem hohen Leistungspreis verrichtet werden. Zusätzlich müssten die Verwaltungskosten eines solchen Unternehmens einkalkuliert werden. Die Funktion der Lohnunternehmen kann in dieser 
Region viel günstiger durch größere Betriebe und Maschinengemeinschaften, die über freie Maschinenkapazitäten verfügen, realisiert werden. Die professionellen LU können hier künftig eine wesentliche Bedeutung bei Verfahren haben, die die Anwendung spezieller Maschinen und/oder des nötigen Fachwissens voraussetzen. Dabei handelt es sich z.B. um den Pflanzenschutz oder um die Organisation der Zuckerrübenernte.

\subsection{Auswahl zweckmäßiger Maschinen unter Berücksichtigung des Kooperationspotenzials}

Vor dem geschilderten Hintergrund wird im Folgenden die Auswahl zweckmäßiger Maschinen für landwirtschaftliche Betriebe beider Regionen erörtert. Dies wird im Lichte des Zustands der bereits in den Betrieben der Stichprobe vorhandenen technischen Ausstattung, des daraus resultierenden Modernisierungsbedarfs und der potenziellen Chancen der Entwicklung der überbetrieblichen Zusammenarbeit erfolgen.

\section{Ermland - Masuren}

Eine wesentliche Bedeutung in der weiteren Modernisierung der Mechanisierung der untersuchten Betriebe der nördlichen Region wird der Schlepperersatz haben. Wie bereits angesprochen, überwiegen in den Betrieben der Stichprobe Schlepper mittlerer Größe, für die ein hohes Alter und hoher Abnutzungsgrad kennzeichnend sind. Der Schlepperzustand kann als ein beträchtlicher Begrenzungsfaktor beim Einsatz von modernen Geräten und weiterer Modernisierung der Produktionsverfahren angesehen werden. Nicht ohne Bedeutung ist die Tatsache, dass die Mehrheit der untersuchten Betriebe eine, an den Flächengrößen gemessen, überproportionale Motorleistung aufweist. Bei anstehenden Ersatzinvestitionen soll stärker der aus der vorliegenden Flächen- und Produktionsstruktur sowie weiteren Entwicklungsplänen resultierende Bedarf an Technik bedacht werden. Eine gemeinsame Anschaffung und anschließender Einsatz von Schleppern in zwei oder drei benachbarten Betrieben scheint in der Befragungsregion generell zu radikal zu sein. Abgesehen von den technischen Aspekten wäre diese Form der Zusammenarbeit auch wegen der mit dem Verzicht auf einen eigenen Schlepper verbundenen Vorbehalten der Landwirte nicht realisierbar. Ein wesentlicher Modernisierungseffekt kann jedoch durch eine Interessenkopplung von Betrieben unterschiedlicher Produktionsstruktur erzielt werden. Als Beispiel hierzu kann eine Zusammenarbeit von Rinderhaltungsbetrieben und Ackerbaubetrieben angeführt werden. In den Betrieben dieser Stichprobe, die eine weitere Spezialisierung im Veredlungsbereich anstreben, kann eine Anschaffung von Schleppern mit einer Motorleistung von über 90 PS wirtschaftlich nicht gerechtfertigt werden. Von der 
Bereitschaft zur Zusammenarbeit beim Schleppereinsatz ausgehend, können für einige wenige Verfahren, bei denen in diesen Betrieben größere und leistungsfähigere Maschinen benötigt werden, die Schlepper der flächenmäßig größeren Ackerbaubetriebe herangezogen werden.

Ferner ist in den untersuchten Betrieben ein Modernisierungsbedarf bei der Grassilagebereitung zu erkennen. In der Mehrheit der untersuchten Betriebe handelt es sich dabei um ein relativ einfaches Verfahren, in dem das Futter nach der Futterwerbung mit einem Silierwagen gehäckselt, geladen und zum Silo gebracht wird. Eine wesentliche Bedeutung bei diesen Arbeiten kommt der Nachbarschaftshilfe zu. Es handelt sich dabei um den Einsatz von Silierwagen sowie Walz- und Transportschleppern. Die Arbeiten werden i.d.R. mit kleinen und zum Teil ausgedienten Maschinen erledigt. Beim Mähen kommen Trommelmäher mit zwei Trommeln zur Anwendung. Beim Häckseln und Transport werden Silierwagen mit einem Ladevolumen von weniger als $15 \mathrm{~m}^{3}$ eingesetzt. Die Modernisierung dieses Arbeitsgangs kann durch Anwendung von größeren leistungsfähigeren Maschinen erzielt werden. Es handelt sich dabei vornehmlich um Silierwagen mit einem Ladevolumen von ca. $26 \mathrm{~m}^{3}$. Eine Alternative für die derzeitige Eigenmechanisierung bietet die Organisation einer Silierkette in einer Maschinengemeinschaft von drei oder vier benachbarten Landwirten.

Des Weiteren ist in den untersuchten Betrieben ein Verbesserungsbedarf im Bereich des Mähdrusches deutlich. Ähnlich wie im Fall der Schlepper sind die meisten Mähdrescher veraltet und weitgehend verschlissen. Die zzt. in der Mehrheit der Untersuchungsbetriebe bewirtschafteten Getreideanbauflächen sichern in Eigenmechanisierung keine erforderliche Auslastung für schlagkräftige moderne Mähdrescher. Diesen Problemen kann mit einer Maschinengemeinschaft begegnet werden. Als Beispiel hierzu dient eine Maschinengemeinschaft von drei Landwirten, die einen gemeinsamen Mähdrescher mit einer Motorleistung von 200 PS in eigenen Betrieben mit einer Funktion der zusätzlichen Dienstleistung bei Dritten einsetzten.

\section{Region Lublin}

Ähnlich wie in der Untersuchungsregion Ermland-Masuren besteht auch im Fall der untersuchten Betriebe in Lublin ein wesentlicher Modernisierungsbedarf beim Schleppereinsatz. Die gemeinsame Anschaffung und ein weiterer Einsatz von Schleppern in zwei bzw. drei benachbarten Betrieben scheinen derzeit eher nicht durchsetzbar zu sein. Dabei treffen die bereits im Fall der nördlichen Region angesprochenen Argumente zu. Der 
Wandel in der Betriebsgrößenstruktur legt eine Veränderung der technischen Ausstattung und den Einsatz neuerer Maschinen nahe. Die Investitionen in neuere und leistungsfähigere Schlepper sind aus wirtschaftlicher Sicht eigentlich nur in den größeren Betrieben der Stichprobe angebracht. In den kleineren Betrieben, in denen kein weiteres Wachstum angestrebt wird, sollen für die relativ einfachen Arbeiten nach wie vor alte Maschinen angewendet werden. In einigen wenigen Verfahren, in denen größere Schlepper benötigt werden, kann die Ausstattung der benachbarten größeren Betriebe zum Zuge kommen. Dementsprechend kann sich künftig die Zusammenarbeit beim Schleppereinsatz entweder in einer den Betrieben der nördlichen Region ähnlichen Form oder auf der Basis der entgeltlichen Dienstleitung entwickeln.

Des Weiteren ist ein Modernisierungsbedarf im Bereich der Zuckerrübenernte zu erkennen. Die Zuckerrüben werden zzt. im Wesentlichen im Rahmen von Eigenmechanisierung gerodet. Zum Einsatz kommen dabei zweireihige angehängte Bunkerköpfroder. Bei Betrieben, die über keine entsprechenden Maschinen verfügen, werden die Erntearbeiten im Rahmen der Nachbarschaftshilfe erledigt. Es handelt sich dabei i.d.R. um unentgeltliche Maschinenausleihung. Die Rüben werden entweder selbständig durch einzelne Landwirte zu einer ca. $20 \mathrm{~km}$ entfernten Zuckerfabrik in Lublin geliefert, oder durch diese Fabrik in Zusammenarbeit mit Speditionsfirmen vom Schlag abgeholt. Bei der Organisation des Transports durch die Zuckerfabrik werden im Vorfeld die Liefermengen sowie die Abholtermine festgelegt. Die Transportkosten werden durch einen entsprechenden Abschlag bei dem Zuckerrübenpreis abgerechnet. Ein Modernisierungseffekt bei diesem Verfahren könnte durch die Organisation einer Arbeitskette von Rübenroden einschließlich Transport in einer Gemeinschaft von einigen Landwirten erzielt werden. Die Leistungskapazitäten der gemeinsamen Maschinen sollen dem aus den Rübenanbauflächen der beteiligten Landwirte resultierenden Bedarf angepasst werden. Demzufolge kann z.B. ein sechsreihiger Selbstfahrer, wegen kleiner Schlaggrößen als auch des hohen Kapitalbedarfs der Investition nicht als eine Alternative für die bestehende Eigenmechanisierung angesehen werden. Der Leistungsbedarf einer Maschinengemeinschaft für die Rübenernte kann mit einem dreireihigen angehängten Rübenköpfroder mit einem Bunkervolumen von ca. $12 \mathrm{~m}^{3}$ gedeckt werden. In einer solchen Gemeinschaft können zunächst, ohne teure Investitionen vornehmen zu müssen, weiterhin die bereits vorhandenen Schlepper und Anhänger eingesetzt werden. Mit weiterer Änderung der Rahmenbedingungen des Zuckerrübenanbaus, insbesondere bei dem Bedarf die Rüben über weitere Entfernungen zu transportieren, kann hier die Entwicklung einer Logistikkette mit dem Einsatz leistungsfähiger Ausstattung an Bedeutung gewinnen. 
Eine wesentliche Bedeutung in der weiteren Entwicklung der Mechanisierung der Betriebe dieser Region wird auch die Modernisierung des Mähdrusches haben. Soweit eine gemeinsame Anschaffung und anschließender Einsatz von Mähdreschern in mehreren kleinen Betrieben allein aus organisatorischen Gründen nicht als realistisch angesehen werden kann, ist ein Zusammenschluss von zwei größeren Betrieben, die einen Mähdrescher mit einer Motorleistung von 130 PS gemeinsam kaufen und einsetzen, denkbar. Die vorhandenen freien Kapazitäten eines solchen Mähdreschers können durch die Dienstleistung in dritten Betrieben ausgelastet werden.

\subsection{Modellkalkulationen}

\subsubsection{Reparaturkostenverlauf}

Der Reparaturkostenverlauf kann einen von Betrieb zu Betrieb unterschiedlichen Charakter annehmen. In der gesamten Nutzungsdauer bestimmter Maschinen gibt es unterschiedlich lange Perioden mit einem unterschiedlich hohen Reparaturaufwand. Bei Bestimmung der Reparaturkosten (RK) ist man stärker als in anderen Fällen auf allgemeine Schätzungen angewiesen.

In Deutschland werden diese Kosten in Anlehnung an die Daten für Betriebsplanungen vom KTBL berechnet. Es wird hier ein über die gesamte Nutzungsdauer in Jahren $(\mathrm{N})$ und der Leistungseinheiten (n) der Maschinen und Anlagen gleichmäßig verteilter Reparaturkostenaufwand angegeben. Es besteht jedoch auch eine Möglichkeit, mithilfe von sog. Anpassungsfaktoren, die Reparaturkosten von bestimmten Nutzungsstadien ausgehend, bis zum Ende der voraussichtlichen Nutzungsdauer annäherungsweise zu berechnen. Diese Faktoren bieten eine Orientierungshilfe für die Ermittlung der Reparaturkosten bei Anschaffung gebrauchter Maschinen, bei denen der Reparaturaufwand üblicherweise höher ist als bei Maschinen, die nur in einer ersten Phase ihrer Nutzungskapazität eingesetzt und anschließend verkauft werden. Mit dieser Vorgehensweise wird versucht, die Progression der Reparaturkosten in Abhängigkeit vom zunehmenden Abnutzungsgrad abzubilden. Trotz der Anpassungsfaktoren kann mithilfe der Kalkulationsdaten der gesamte Reparaturkostenverlauf nicht dargestellt werden.

In Polen wird für die Ermittlung der Reparaturkosten der Landmaschinen ein Koeffizient herangezogen, der prozentual eine Relation des gesamten Reparaturaufwands (Kosten der Ersatzteile und Lohnansatz) während der gesamten Nutzungsdauer zu dem Anschaffungswert 
einer Maschine widerspiegelt. Der Koeffizient kann in Abhängigkeit von Maschinenart von 0,4 bis 1,5 reichen. Die Reparaturkosten pro $\mathrm{h}$ errechnet man aus folgender Formel (vgl. MuZALEWSKI 2004, S. 8 ff.):

$R K=\frac{K \times A}{n}$

dabei bedeuten:

K- Koeffizient,

A- Anschaffungspreis,

$\mathrm{n}$ - Nutzungsdauer in Leistungseinheiten in h oder ha.

Diese Methodik wird im Hinblick auf ihre Zuverlässigkeit oft kritisiert. Es wird dabei vorgetragen, dass die Datengrundlagen unter Bedingungen großer staatlicher Landesunternehmen in den achtziger Jahren herausgearbeitet wurden. Aufgrund der wesentlichen Änderungen der Leistungsfähigkeit und der Verhältnisse der Reparaturkosten zu dem Maschinenanschaffungspreis werden die unterstellten Daten oft als Ursache weitgehender Ungenauigkeit in der Erfassung der Kosten des Maschineneinsatzes angesehen (vgl. LORENCOWICZ 1997, S. 75).

\subsubsection{Restwertermittlung}

Unter dem Restwert (RW) wird im engen Sinn der sich aus der Angebots- und Nachfragerelation auf dem regionalen Gebrauchtmaschinenmarkt ergebende Verkaufspreis einer Maschine zum Zeitpunkt des Endes ihrer Nutzungsdauer verstanden. Dem Restwert einzelner Maschinen kann eine wesentliche Bedeutung in den Investitionsentscheidungen zukommen. Bei der Ermittlung dieser Größe können als Orientierungshilfe verschiedene Datenquellen herangezogen werden. Eine besondere Bedeutung haben in der Praxis in Deutschland die Veröffentlichungen von Eurotax-Schwacke. In der sog. Schwackeliste werden Notierungen für bestimmte Maschinen mit einer durchschnittlichen, in Betriebsstunden angegebenen, Leistung dargestellt. Es wird beispielhaft bei Schleppern von einer jährlichen Leistung von 600 h, bei Mähdreschern von $200 \mathrm{~h}$ und bei Häckslern von $300 \mathrm{~h}$ ausgegangen. Bei evtl. Mehr- oder Minderleistung sind entsprechende prozentuale Korrekturen vorzunehmen. In diesen Notierungen wird zwischen Preisen, die die Landwirte beim Verkauf bestimmter Maschinen erzielen und den Preisen, die sie beim Kauf gebrauchter Maschinen bei Händlern bezahlen, differenziert. Diese Datenquelle kann allerdings nur als eine grobe Orientierungshilfe herangezogen werden. Diese Feststellung gründet sich u.a. 
darin, dass die in den Notierungen dargestellten Werte die regionalen Disparitäten in der Entwicklung der Verkaufspreise nicht aufzuzeigen vermögen ${ }^{28}$.

\subsubsection{Datenquellen}

Für die Kostenkalkulation des Maschineneinsatzes liegen zwei bereits erwähnte Datenquellen vor. Es handelt sich dabei zum einen um die in Polen gültigen Kalkulationsdaten vom IBMER und zum anderen um die in Form eines Standardwerkes periodisch veröffentlichte Datensammlung für Betriebskalkulationen vom KTBL. Ausgehend von der in beiden Quellen unterstellten Nutzungsdauer in Jahren können die voraussichtlichen je Leistungseinheit anfallenden Kosten des Maschineneinsatzes berechnet werden.

Bei den Daten vom IBMER wird eine Nutzungsdauer von 20 Jahren für Schlepper und von 18 Jahren für Mähdrescher unterstellt. Dies scheint für die Kalkulationen künftiger Mechanisierung wesentlich zu lang zu sein. Aufgrund dieser Tatsache und wegen der oben angesprochenen Kritiken an der Zuverlässigkeit dieser Daten werden in weiteren Kalkulationen die Daten vom KTBL angewendet. Dabei sind allerdings einige Modifikationen erforderlich. Die üblicherweise in Deutschland unterstellte N von 12 Jahren für Schlepper und 10 Jahren für Mähdrescher kann unter polnischen Verhältnissen nicht unterstellt werden. Um den realen Bedingungen angemessen Rechnung zu tragen, wird im Folgenden für Schlepper und Mähdrescher eine einheitliche N von 15 Jahren angenommen. Der voraussichtliche Nutzungsumfang in Leistungseinheiten ausgewählter Maschinen wird unverändert aus den KTBL - Daten entnommen. Dies hat zur Folge, dass bei der für weitere Kalkulationen verlängerter $\mathrm{N}$ die Abschreibungsschwelle im Vergleich zu den deutschen Daten herabgesetzt wird.

Eine weitere Modifizierung betrifft die Reparaturkosten. Die in den KTBL-Daten unterstellten Reparaturkosten enthalten u.a. den Lohnansatz der technischen Fachkräfte. Bei der vom KTBL für die Datengewinnung angewendeten Methodik ist es nicht möglich, diese Kostenposition getrennt auszuweisen (WEIERSHÄUSER). Wegen der zwischen Deutschland und Polen bestehenden Unterschiede im Lohnniveau können die Reparaturkosten nicht direkt vom KTBL übernommen werden. Daher wird zunächst die Proportion des Anschaffungspreises (A) zu den innerhalb der gesamten $\mathrm{N}$ entstehenden Reparaturkosten bestimmter Maschinen berechnet.

\footnotetext{
${ }^{28}$ Ausführlich zur Bewertung der Landmaschinen in Deutschland siehe Köhne 2000, S.472 ff.
} 
Die Formel hierzu kann folgendermaßen geschrieben werden:

$$
X=\frac{R K}{A}
$$

Für die Ermittlung der Reparaturkosten unter polnischen Bedingungen werden unter Berücksichtigung der realen Anschaffungspreise bestimmter Maschinen auf dem polnischen Markt die, mithilfe der angegebenen Formel aus den KTBL - Daten abgeleiteten Proportionen herangezogen. Die Anschaffungspreise, die für die analysierten Maschinen unterstellt werden, stammen aus den Informationen von Fachzeitschriften, Landmaschinenherstellern sowie aus einer eingeschränkten Befragung der Landmaschinenhändler in Polen. Dieselbe Quelle wird auch für die Kalkulationen der Preise bei Inanspruchnahme der Lohnmaschinenleistungen in den analysierten Arbeitsgängen herangezogen. Schließlich werden die Unterbringungs- und Versicherungskosten in Anlehnung an die Daten vom IBMER berechnet.

\subsubsection{Kalkulationsmethodik}

Die durchschnittlichen Maschinenkosten (DK) werden nach approximativer Kalkulation als Summe der durchschnittlichen jährlichen Abschreibung, des durchschnittlichen Zinsanspruchs (Zi), der durchschnittlichen Reparaturen und der jährlich anfallenden Unterbringungs- und Versicherungskosten berechnet (vgl. BRANDES, ODENING 1992, S. 40 ff.).

Für die Berechnung der Abschreibung ist der Auslastungsgrad der Maschinen von Bedeutung. Unter Berücksichtigung der sog. Abschreibungsschwelle, die dann erreicht wird, wenn die jährliche Auslastung (j) in h oder ha der Nutzungsdauer in Leistungseinheiten in $\mathrm{h}$ oder ha durch die Nutzungsdauer in Jahren gleich ist, also $\mathrm{j}=\frac{n}{N}$ werden zwei Fälle unterschieden.

Fall A. Die jährliche Auslastung liegt unterhalb der Abschreibungsschwelle, also $\mathrm{j}<\frac{n}{N}$ und die Abschreibung wird zu festen Kosten gezählt, gilt:

$$
A f A=\frac{A-R W}{N}
$$

Fall B. Die jährliche Auslastung liegt oberhalb der Abschreibungsschwelle, also $\mathrm{j}>\frac{n}{N}$ und die Abschreibung wird zu variablen Kosten gezählt, gilt:

$$
A f A=\frac{A-R W}{n}
$$


Für die Ermittlung des durchschnittlichen Zinsanspruchs $\left(\mathrm{Z}_{\mathrm{i}}\right)$ wird folgende Gleichung zugrunde gelegt:

$Z i=(A+R W) \times 0,5 \times i$

dabei bedeutet:

$i$ - Zinssatz.

Die Formel für die Ermittlung der durchschnittlichen Maschinenkosten kann zusammenfassend wie folgt beschrieben werden:

Fall A. $\mathrm{j}<\frac{n}{N}$

1. Feste Kosten (je Jahr)

$\frac{A-R W}{N}+((A+R W) \times 0,5) \times i+U+V$

2. Variable Kosten (je ha oder h)

$\frac{\sum R K}{N \times j}+$ Betriebsstoffe

Fall B. $\mathrm{j}>\frac{n}{N}$

1. Feste Kosten (je Jahr)

$((A+R W) \times 0,5) \times i+U+V$

2. Variable Kosten (je ha oder h)

$\frac{A-R W}{n}+\frac{\sum R K}{n}+$ Betriebsstoffe

In allen Kalkulationen wird einheitlich ein RW von $10 \%$ des Anschaffungspreises berücksichtigt. Ferner wird angenommen, dass die Investitionen überwiegend mithilfe eines Kredits finanziert werden. Daher wird, gemäß den aktuellen Informationen über Kreditverleihe aus den polnischen Banken, einheitlich ein Zinssatz von $6 \%$ unterstellt. Die Währung aller folgenden Kalkulationen ist der polnische ZL. Die unterstellten Maschinenanschaffungspreise enthalten eine Umsatzsteuer (USt) von $22 \%$.

Vorgehensweise bei den Kostenkalkulationen der bisherigen Ausstattung

Für die Ermittlung der festen Kosten der zzt. in den untersuchten Betrieben befindlichen Maschinen wird einheitlich eine lineare Abschreibung von ihrem Marktwert (MW) ausgehend vorgenommen. Die unterstellte Restnutzungsdauer (RN) der Maschinen entspricht den Schätzungen der befragten Landwirte. Aus Vereinfachungsgründen wird angenommen, dass die Maschinen am Ende der Nutzungsdauer keinen Restwert haben. Der Zinssatz beträgt in 
den Kalkulationen $6 \%$. Bei der Ermittlung der variablen Kosten wird der zeitliche Anstieg der Reparaturauszahlungen nicht explizit berücksichtigt. Es wird mit durchschnittlichen jährlichen Reparaturkosten während der RN gerechnet. Sie werden in der Höhe angesetzt, wie sie in der Befragung von den Landwirten angegeben wurden. Für die Ermittlung der Kosten der Betriebsstoffe sowie der Unterbringungs- und Versicherungskosten wird auf die Kalkulationsdaten vom IBMER zurückgegriffen.

Es kann zusammenfassend folgende Formel geschrieben werden:

$D K=\frac{M W}{R N}+(M W \times 0,5) \times i+\frac{\sum R K}{R N}+U+V+$ Betriebsstoffe (je Jahr)

Bei der Dividierung der so ermittelten Kosten durch die jährlichen Auslastungen ergeben sich die Kosten je h oder ha. Die Kalkulationsergebnisse enthalten die Tabellen 5-10 sowie 15-20 im Anhang A.5.

\subsubsection{Kalkulationen des Einsatzes neuer und gebrauchter Maschinen}

In Bezug auf die vorangegangenen Ausführungen werden an dieser Stelle die Kosten des Einsatzes bestimmter Maschinen in den Produktionsverfahren Getreideernte, Silagebereitung und Zuckerrübenernte analysiert.

\section{Schlepper}

Eine wesentliche Bedeutung für die Entwicklung der Zusammenarbeit in den zwei letzten Verfahren hat der gemeinsame Schleppereinsatz. Vor diesem Hintergrund werden in der Tabelle 6-2 exemplarisch die Kosten eines Schleppers mit einer Motorleistung von 90 PS dargestellt. Es werden hier zwei Alternativen gegenübergestellt. Die erste Spalte enthält die Kosten eines neuen Schleppers, in der zweiten werden hingegen die Kosten eines gebrauchten Schleppers aufgezeigt. Dabei wird hier und im Folgenden in erster Linie an importierte Maschinen (z.B. aus Deutschland) gedacht. In dieser Tabelle werden zunächst die festen Kosten pro Jahr und die variablen je h berechnet. Anschließend werden sie zu den gesamten Kosten zusammengefasst, indem die festen Kosten durch die jährliche Auslastung dividiert und die variablen Kosten hinzuaddiert werden. Die Reparaturen bei der gebrauchten Maschine werden um $20 \%$ im Vergleich zur ersten Variante angehoben. Dem unteren Teil der Übersicht sind die Gesamtkosten pro Stunde zu entnehmen. Nach dem dargestellten Schema werden alle folgenden Kalkulationen aufgebaut. 
Tabelle 6-2: Kostenkalkulation eines Schleppers mit einer Motorleistung von 90 PS (ZL)

\begin{tabular}{|c|c|c|c|c|c|c|c|}
\hline Datengrundlagen & \multicolumn{4}{|c|}{ Neuer Schlepper } & \multicolumn{3}{|c|}{ Gebrauchter Schlepper (5 Jahre alt) } \\
\hline Anschaffungspreis & \multicolumn{4}{|c|}{140000} & \multicolumn{3}{|c|}{70000} \\
\hline Nutzungsumfang in $\mathrm{N} / \mathrm{n}$ & \multicolumn{4}{|c|}{15 Jahre/ $10000 \mathrm{~h}$} & \multicolumn{3}{|c|}{10 Jahre/ $6400 \mathrm{~h}$} \\
\hline Restwert & \multicolumn{4}{|c|}{14000} & \multicolumn{3}{|c|}{14000} \\
\hline \multicolumn{8}{|c|}{ Feste Kosten/ Jahr } \\
\hline AfA & \multicolumn{4}{|c|}{8400} & \multicolumn{3}{|c|}{5600} \\
\hline Zinsanspruch & \multicolumn{4}{|c|}{4620} & \multicolumn{3}{|c|}{2520} \\
\hline $\begin{array}{l}\text { Unterbringung und } \\
\text { Versicherung }\end{array}$ & \multicolumn{4}{|c|}{600} & \multicolumn{3}{|c|}{600} \\
\hline Summe feste Kosten & \multicolumn{4}{|c|}{13620} & \multicolumn{3}{|c|}{8720} \\
\hline \multicolumn{8}{|c|}{ Variable Kosten/ h } \\
\hline Reparaturen & \multicolumn{4}{|c|}{13} & \multicolumn{3}{|c|}{15} \\
\hline Betriebsstoffe $^{29}$ & \multicolumn{4}{|c|}{31} & \multicolumn{3}{|c|}{31} \\
\hline Summe variable Kosten & \multicolumn{4}{|c|}{44} & \multicolumn{3}{|c|}{46} \\
\hline \multicolumn{8}{|c|}{ Gesamte Kosten } \\
\hline Einsatzumfang in $\mathrm{h} / \mathrm{Jahr}$ & 200 & 400 & 600 & $800^{30}$ & 200 & 400 & 600 \\
\hline Feste Kosten pro h & 68 & 34 & 23 & 17 & 44 & 22 & 15 \\
\hline Variable Kosten pro h & \multicolumn{4}{|c|}{44} & \multicolumn{3}{|c|}{46} \\
\hline Summe pro $\mathrm{h}$ & 112 & 78 & 67 & 61 & 90 & 68 & 61 \\
\hline
\end{tabular}

Quelle: eigene Darstellung

Wie die dargestellte Kalkulation zeigt, ist der Einsatz der gebrauchten Maschine aus der Kostensicht günstiger. Die Kostendegression ist besonders deutlich bei niedrigerer Auslastung und schwächt sich parallel zu dem steigenden Einsatzumfang ab.

\section{Mähdrescher 200 PS}

In der Tabelle 6-3 werden die Kosten eines Mähdreschers mit einer Motorleistung von 200 PS und einer Arbeitsbreite des Schneidwerks von 4,5 Metern erläutert. Wegen der Unterschiede im Anschaffungspreis ist der Einsatz der Gebrauchtmaschine wesentlich billiger. Der Kostenvorsprung nimmt zwar mit der steigenden Auslastung ab, die gebrauchte Maschine bleibt jedoch bis zur Abschreibungsschwelle, die in diesem Fall bei $200 \mathrm{~h} / \mathrm{Jahr}$ liegt, um zumindest ein Viertel billiger als der neue Mähdrescher. Die zahlenmäßig dargestellte Annahme kann leicht aufgehoben werden. Bei einem stärkeren Anstieg der Reparaturausgaben können die Kostenvorteile bei der Arbeitserledigung mit gebrauchten Maschinen wesentlich beeinträchtigt werden. Unter diesen Bedingungen müsste zusätzlich das erhöhte Ausfallrisiko bei unerwartet auftretenden Reparaturen berücksichtigt werden. Vorbehaltlich dessen wurden, v.a. wegen prägnanter Kostenvorteile für das konkrete Beispiel einer Maschinengemeinschaft, die Kosten des gebrauchten Mähdreschers herangezogen.

\footnotetext{
${ }^{29}$ Die Kosten der Betriebsstoffe setzen sich aus den Kosten für Diesel und Schmieröl zusammen. Es wird in allen Kalkulationen einheitlich ein Preis für $1 \mathrm{~L}$ Diesel von 3,9 ZL und für $1 \mathrm{~L}$ Schmieröl von 14 ZL unterstellt. Der Betriebsstoffverbrauch für jede einzelne Maschine wird in Anlehnung an KTBL berechnet.

${ }^{30}$ Obwohl die hier unterstellte Auslastung oberhalb der Abschreibungsschelle liegt, wird die AfA zur Wahrung der Übersichtlichkeit vereinfacht zu festen Kosten gezählt. Dies gilt auch für alle folgenden Kalkulationen.
} 
Der in der Kalkulation für bestimmte Getreidearten unterstellte Zeitbedarf in $\mathrm{h} / \mathrm{ha}$ wird in Anlehnung an die KTBL-Daten zur Betriebsplanung für einen 2 ha großen Schlag mittelschweren Bodens berechnet. Der jährliche Einsatz des Mähdreschers von insgesamt 146 h sichert nicht die erforderliche Auslastung. Die vorhandenen freien Kapazitäten können zusätzlich in benachbarten Betrieben verwendet werden. Solange die zusätzliche Auslastung nicht über die voraussichtliche Abschreibungsschwelle hinausgeht und die Nutzungsdauer des Mähdreschers nicht verringert wird, bleiben die festen Kosten unverändert. Somit errechnet sich das Zusatzeinkommen als Differenz zwischen dem erzielbaren Verrechungswert und den zusätzlich anfallenden variablen Kosten zuzüglich des Lohnansatzes. In der Tabelle 6-5 werden die Kosten und die zusätzlichen Einnahmen bei einer um 50 ha (50 h) erweiterten Auslastung des Mähdreschers in anderen Betrieben aufgezeigt. Für dieses Beispiel wird ein Preis der Dienstleistung von $270 \mathrm{ZL} /$ ha unterstellt. Unter Berücksichtigung der variablen Kosten einschließlich eines Lohnansatzes von $18 \mathrm{ZL} / \mathrm{h}$ wird in den Mitgliedsbetrieben ein Überschuss von 6.350 ZL generiert. Dieser Betrag wird analog zu der früheren Kalkulation nach dem Maßstab der Anteile an den Anschaffungskosten unter den Gemeinschaftsmitgliedern aufgeteilt. Um den Einfluss der zusätzlichen Auslastung auf die gesamten Maschineneinsatzkosten in den Mitgliedsbetrieben zu verdeutlichen, werden im unteren Teil dieser Tabelle die erzielbaren Einkommen mit den ursprünglichen gesamten Maschineneinsatzkosten pro Betrieb aus der Tabelle 6-4 verrechnet. Der zusätzliche Einsatz des Mähdreschers führt zu einer Reduktion der gesamten Kosten in den Mitgliedsbetrieben von $278 \mathrm{ZL} /$ ha auf $234 \mathrm{ZL} / \mathrm{ha}$.

Tabelle 6-5: Verteilung des durch eine zusätzliche Auslastung des Mähdreschers mit 200 PS erzielbaren Einkommens (ZL)

\begin{tabular}{|c|c|c|}
\hline Einsatzumfang & \multicolumn{2}{|c|}{$70 \mathrm{ha} \mathrm{LF} / 70 \mathrm{~h}$} \\
\hline Variable Kosten: & \multirow{2}{*}{\multicolumn{2}{|c|}{1550}} \\
\hline Reparaturen & & \\
\hline Betriebsstoffe & \multicolumn{2}{|c|}{4700} \\
\hline Lohnansatz & \multicolumn{2}{|c|}{900} \\
\hline Gesamte Kosten & \multicolumn{2}{|c|}{7150} \\
\hline Einnahmen & \multicolumn{2}{|c|}{13500} \\
\hline Differenz & \multicolumn{2}{|c|}{6350} \\
\hline Betrag pro Betrieb & \\
\hline I & \multicolumn{2}{|c|}{1778} \\
\hline II & \multicolumn{2}{|c|}{2095} \\
\hline III & \multicolumn{2}{|c|}{2477} \\
\hline & Gesamte Kosten pro Betrieb & Gesamte Kosten pro ha \\
\hline I & 9493 & \\
\hline II & 11023,5 & 234 \\
\hline III & 13333,5 & \\
\hline
\end{tabular}


Bei einer erheblichen dauerhaften Änderung der Anteile der Mähdruschflächen in einzelnen Mitgliedsbetrieben müssten die Kosten neu berechnet werden. In der Tabelle 6-6 wird ein Beispiel dargestellt, in dem der Betrieb I nach drei Jahren aus der Gemeinschaft austritt und die übrigen zwei Betriebe die Abfindungssumme gemeinsam aufbringen. Es wird unterstellt, dass der Marktwert des Mähdreschers zu diesem Zeitpunkt 110.000 ZL beträgt. Nach der Verrechung dieses Wertes in den Mitgliedsbetrieben, haben die verbleibenden zwei Landwirte zugunsten des ausscheidenden Landwirts einen Abfindungsbetrag von insgesamt $30.800 \mathrm{ZL}$ zu entrichten. Die Änderung der Mitgliederanzahl wirkt sich auf die Kosten der Arbeitserledigung pro ha Einsatzfläche aus. Bei einer Auslastung des Mähdreschers in den zwei Mitgliedsbetrieben, ohne Berücksichtigung einer zusätzlichen Dienstleistung, steigen die Kosten im Vergleich zu der Gemeinschaft mit drei Landwirten auf 337 ZL/ha.

Tabelle 6-6: Umverteilung der Kosten einer Maschinengemeinschaft beim Austritt eines Mitglieds (ZL)

\begin{tabular}{|c|c|c|c|}
\hline \multicolumn{2}{|c|}{ Beteiligter Landwirt } & Anteil & Abfindungssumme \\
\hline & I & 30800 & +30800 \\
\hline & II & 36300 & -14168 \\
\hline & III & 42900 & -16632 \\
\hline & feste Kosten pro Jahr & variable Kosten pro 104 ha oder 105 ha & Gesamte Kosten pro ha \\
\hline & 21950 & 13125 & 337 \\
\hline & & Gesamte Kosten pro Betrieb & \\
\hline I & & $48 \times 337=16176$ & \\
\hline II & & $56 \times 337=18872$ & \\
\hline
\end{tabular}

Quelle: eigene Darstellung

Aus den Beispielsrechungen lassen sich einige Schlussfolgerungen ableiten. Der relativ kleine Umfang der Mähdruschflächen in der exemplarischen Maschinengemeinschaft spricht für den Einsatz eines gebrauchten Mähdreschers. Soweit eine gebrauchte Maschine nicht sehr stark abgenutzt ist und ihr Einsatz keine erhebliche Progression des Reparaturaufwands und somit kein besonderes Ausfallrisiko verursacht, ist sie aus Kostensicht wesentlich günstiger als ein neuer Mähdrescher. Wird bei der Kostenrechung des im Beispiel dargestellten gebrauchten Mähdreschers auch die Alternative der Inanspruchnahme einer Maschinenleistung eines Lohnunternehmers zu einem Preis von $270 \mathrm{ZL} / \mathrm{ha}$ berücksichtigt, so zeigt sich, dass die gemeinsame Maschine, unter Berücksichtigung eines Lohnansatzes für die AK von $18 \mathrm{ZL} / \mathrm{h}$ erst ab einer Mindestauslastung von 175 ha/Jahr niedrigere Kosten als die Inanspruchnahme der Lohnmaschinenleistung verursacht. Bei dem neuen Mähdrescher verschiebt sich der Mindestauslastungsgrad auf $290 \mathrm{ha} / \mathrm{Jahr}$ (siehe Abbildung 6-1). In Betrieben, in denen die Bedingung der Mindestauslastung nicht erfüllt ist, können die Maschineninvestitionen bei ausreichendem Angebot der Fremdmaschinenleistung im Sinne der Kosten nicht 
gerechtfertigt werden. Darüber hinaus ist an dieser Stelle auch die Entlohnung der eigenen Arbeit zu berücksichtigen.

Abbildung 6-1: Kosten des Einsatzes des neuen und gebrauchten Mähdreschers mit 200 PS in Abhängigkeit vom Leistungsumfang (ZL)

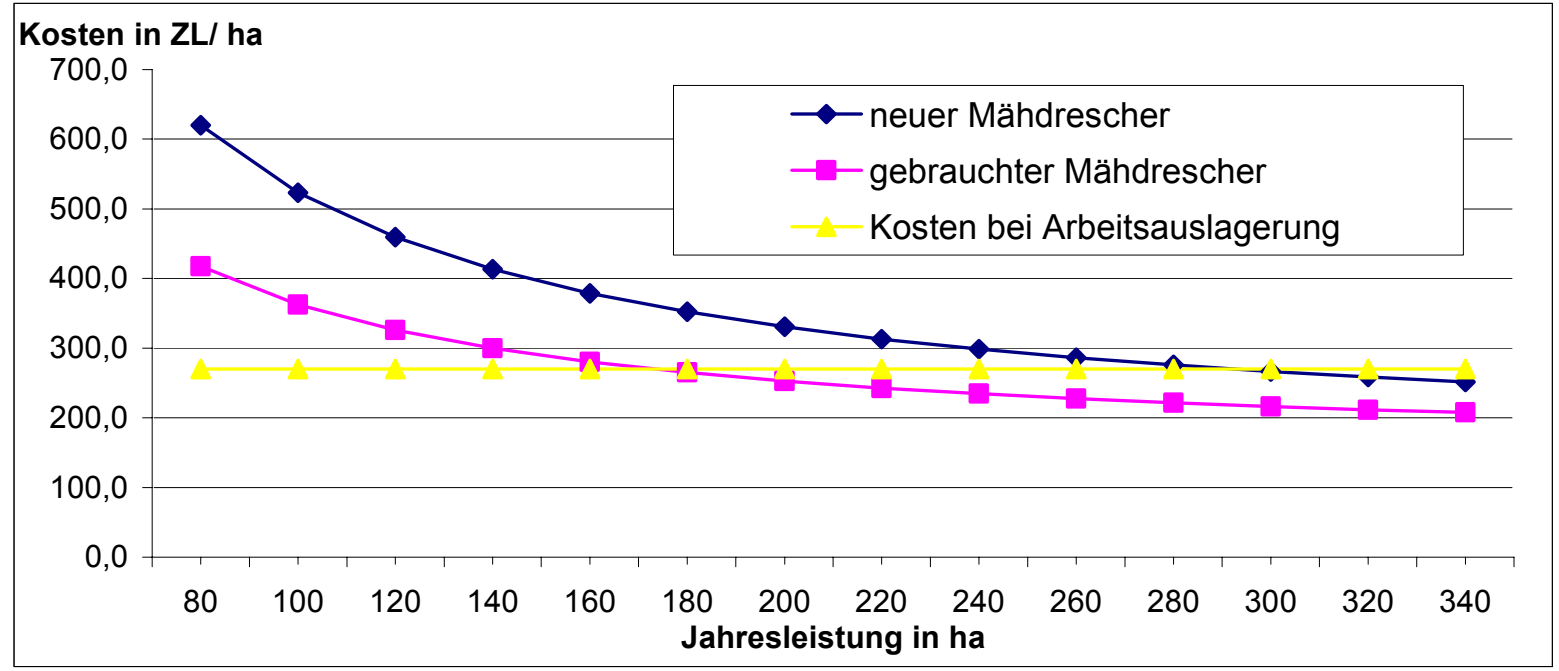

Quelle: eigene Darstellung

Von der dargestellten Beispielsrechung ausgehend, kann eine vereinfachte Analyse der Kosten der Mechanisierung in einer Maschinengemeinschaft und bei Inanspruchnahme der Lohnmaschinenleistung vorgenommen werden. Die Daten zu diesem Vergleich werden in der Tabelle 6-7 zusammengestellt. Es werden hier die Kosten des Mähdrusches in der zunächst dargestellten Zusammenarbeit von drei Landwirten mit der zusätzlichen Auslastung des Mähdreschers $(50 \mathrm{~h})$ in anderen Betrieben dargestellt. Die erste Spalte gibt die gesamten Kosten wieder, die jeder der drei Landwirte für den Mähdrusch auf eigenen Flächen trägt. Es wird hier ebenso wie im Beispiel der Abbildung 6-1 ein Lohnansatz in Höhe von 18 ZL/h einkalkuliert. Beim Vergleich der Kosten der Maschinengemeinschaft mit der Arbeitsauslagerung ist die erste Mechanisierungsform kostengünstiger. Die in jedem der Mitgliedsbetriebe durch die Zusammenarbeit eingesparten Beträge, sind in der dritten Zeile ausgewiesen. Dieses Ergebnis kann weiter modifiziert werden, wenn für die Anschaffung der Maschine statt mit Fremdkapital aus eigenen Mitteln finanziert wird. Der Aspekt der Kapitalrendite kann besondere Bedeutung in jenen Betrieben gewinnen, in denen den Maschineninvestitionen hohe Opportunitätskosten z.B. durch innerbetriebliche Entwicklung gegenüberstehen. 
Tabelle 6-7: Kosten des Einsatzes des Mähdreschers in einer Maschinengemeinschaft dreier Landwirte vs. Inanspruchnahme der Lohnmaschinenleistung (ZL)

\begin{tabular}{|c|c|c|c|}
\hline & Landwirt I & Landwirt II & Landwirt III \\
\hline Maschinengemeinschaft & 10231 & 11869,5 & 14377,5 \\
\hline Arbeitsauslagerung & 11070 & 12690 & 15660 \\
\hline Differenz & 839 & 820,5 & 1282,5 \\
\hline
\end{tabular}

Quelle: eigene Darstellung

\section{Mähdrescher 130 PS}

Analog zu der Leitlinie der Kostenrechung des ersten Mähdreschers kann eine Beispielsrechung für den gemeinsamen Einsatz eines kleineren Mähdreschers mit einer Motorleistung von 130 PS aufgebaut werden. In der Tabelle 6-8 werden Varianten der Kosten beim Einsatz eines neuen und eines gebrauchten Mähdreschers aufgezeigt.

Tabelle 6-8: Kostenkalkulation eines Mähdreschers mit einer Motorleistung von 130 PS (ZL)

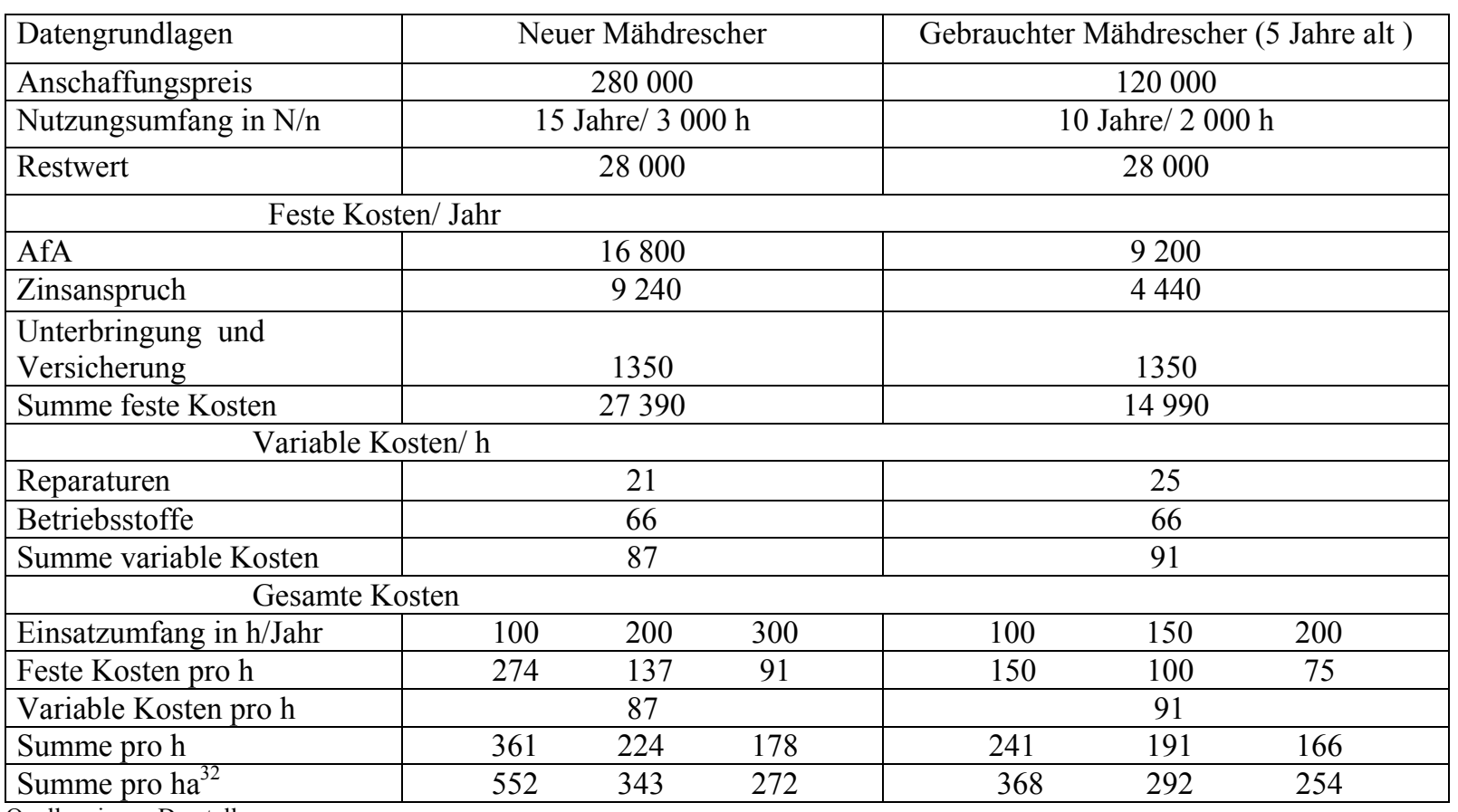

Quelle: eigene Darstellung

Die Aufteilung der Anschaffungs- und Einsatzkosten in einer Gemeinschaft zweier Landwirte mit einem Flächenumfang von 35 ha bzw. 65 ha ist der Tabelle 6-9 zu entnehmen.

\footnotetext{
${ }^{32}$ Es wird hier vereinfacht angenommen, dass die Leistung des Mähdreschers bei einer Schlaggröße von 1 ha 0,65 ha pro h entspricht.
} 
Tabelle 6-9: Verteilung der Anschaffungs- und Einsatzkosten des gebrauchten Mähdreschers mit 130 PS in einer Maschinengemeinschaft zweier Landwirte (ZL)

\begin{tabular}{|c|c|c|c|c|}
\hline $\begin{array}{l}\text { Beteiligter } \\
\text { Landwirt }\end{array}$ & \multicolumn{2}{|c|}{ Einsatzfläche/Arbeitszeit } & \multicolumn{2}{|c|}{ Finanzierungsbeitrag } \\
\hline I & \multicolumn{2}{|c|}{$\begin{array}{c}18 \text { ha Weizen } / 31 \mathrm{~h} \\
15 \text { ha Triticale } / 19 \mathrm{~h} \\
2 \mathrm{ha} \text { Gerste } / 3 \mathrm{~h}\end{array}$} & \multicolumn{2}{|c|}{42000} \\
\hline II & \multicolumn{2}{|c|}{$\begin{array}{l}25 \text { ha Weizen/ } 42 \mathrm{~h} \\
20 \text { ha Raps/ } 32 \mathrm{~h} \\
20 \text { ha Gerste/ } 26 \mathrm{~h}\end{array}$} & \multicolumn{2}{|c|}{78000} \\
\hline Gesamt & \multicolumn{2}{|c|}{$100 \mathrm{ha} / 153 \mathrm{~h}$} & \multicolumn{2}{|c|}{120000} \\
\hline \multirow[b]{4}{*}{$\begin{array}{c}\text { I } \\
\text { II }\end{array}$} & feste Kosten pro Jahr & \multicolumn{2}{|c|}{ variable Kosten pro 100 ha oder $143 \mathrm{~h}$} & Gesamte Kosten pro ha \\
\hline & 14990 & \multicolumn{2}{|c|}{13923} & 289 \\
\hline & \multicolumn{4}{|c|}{ Gesamte Kosten pro Betrieb } \\
\hline & \multicolumn{4}{|c|}{$\begin{array}{l}5246,5+4873=10119,5 \\
9743,5+9050=18793,5\end{array}$} \\
\hline
\end{tabular}

Der in der Tabelle 6-9 unterstellte Zeitbedarf in $\mathrm{h} / \mathrm{ha}$ bei bestimmten Getreidearten wurde in Anlehnung an die KTBL-Daten zur Betriebsplanung für einen 1 ha großen Schlag mittelschweren Bodens berechnet. Die dargestellte Kalkulation kann um die Alternative einer zusätzlichen Auslastung des Mähdreschers auf 50 ha LF in anderen Betrieben erweitert werden. Dazu ist auf die Tabelle 6-10 zu verweisen. Die Vorgehensweise bei der Bemessung des Einflusses der zusätzlichen Auslastung des Mähdreschers auf die Maschinenkosten in den Mitgliedsbetrieben ist gleich der in der Tabelle 6-5. Bei denselben Annahmen wie in dem vorangegangenen Beispiel erzielt diese Gemeinschaft ein zusätzliches Einkommen in Höhe von insgesamt 5.216 ZL. Bei der Verrechung des Betrages mit den Maschineneinsatzkosten in den Mitgliedsbetrieben ergibt sich eine Reduktion der Kosten von 289 ZL/ha auf 237 ZL/ha.

Tabelle 6-10: Verteilung des durch eine zusätzliche Auslastung des Mähdreschers mit 130 PS erzielbaren Einkommens (ZL)

\begin{tabular}{|c|c|c|}
\hline \multirow[b]{2}{*}{\begin{tabular}{|l|} 
Einsatzumfang \\
Variable Kosten \\
Reparaturen \\
Betriebsstoffe \\
Lohnansatz
\end{tabular}} & \multicolumn{2}{|c|}{$50 \mathrm{ha} \mathrm{LF} / 76 \mathrm{~h}$} \\
\hline & \multicolumn{2}{|c|}{$\begin{array}{l}1900 \\
5016 \\
1368\end{array}$} \\
\hline \begin{tabular}{|l} 
Gesamte Kosten \\
\end{tabular} & \multicolumn{2}{|c|}{8284} \\
\hline Einnahmen & \multicolumn{2}{|c|}{13500} \\
\hline Differenz & \multicolumn{2}{|c|}{5216} \\
\hline $\begin{array}{c}\text { Betrag pro Betrieb } \\
\text { I } \\
\text { II }\end{array}$ & \multicolumn{2}{|c|}{$\begin{array}{l}1825,6 \\
3390,4 \\
\end{array}$} \\
\hline & Gesamte Kosten pro Betrieb & Gesamte Kosten pro ha \\
\hline $\begin{array}{l}\text { I } \\
\text { II }\end{array}$ & $\begin{array}{c}8294 \\
15403\end{array}$ & 237 \\
\hline
\end{tabular}

Quelle: eigene Darstellung 
Die dargestellte Kalkulation belegt die Richtigkeit der Auswahl des gebrauchten Mähdreschers für die exemplarische Maschinengemeinschaft. Werden die Kosten beim Einsatz des gebrauchten Mähdreschers mit den Kosten der Arbeitsauslagerung verglichen, so wird deutlich, dass der Mähdrescher wenigstens 145 ha/Jahr eingesetzt werden muss, damit die Kosten der Eigenmechanisierung nicht über den Kosten der Arbeitsauslagerung liegen. Beim Einsatz des neuen Mähdreschers verschiebt sich die Mindestauslastungsgrenze auf 250 ha/Jahr (siehe Abbildung 6-2).

Abbildung 6-2: Kosten des Einsatzes eines neuen und gebrauchten Mähdreschers mit 130 PS in Abhängigkeit vom Leistungsumfang (ZL)

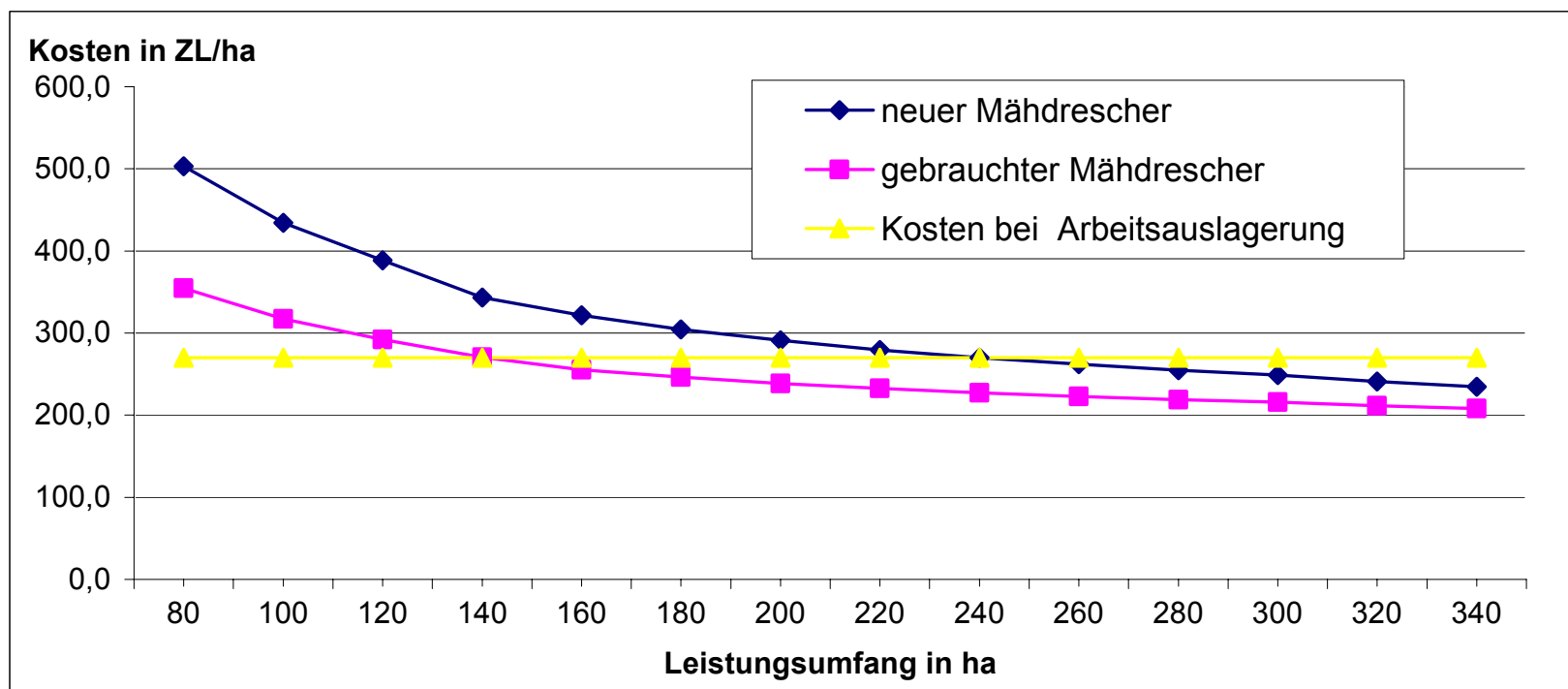

Quelle: eigene Darstellung

\section{Silierwagen}

Ein weiteres Beispiel einer praktikablen Zusammenarbeit betrifft eine Gemeinschaft zweier Landwirte, die einen Silierwagen mit einem Ladevolumen von $26 \mathrm{~m}^{3}$ einsetzen. Die Einsatzflächen der Mitgliederbetriebe bestehen aus jeweils 15 ha, 20 ha und 30 ha. Die Kosten der Anschaffung und des anschließenden Einsatzes der Maschine werden in einer den früheren Beispielen entsprechenden Vorgehensweise analysiert. Dazu wird zunächst auf die Tabelle 6-11 verwiesen, die die einzelnen Kostenpositionen enthält. Analog zu den früheren Beispielen werden hier die gesamten Kosten beider Varianten für einen unterschiedlichen Einsatzumfang berechnet. Die Kosten werden pro eine Tonne (t) Anwelksilage ermittelt. Der Einsatz einer Gebrauchtmaschine ist aus Kostensicht, insbesondere bei niedrigerer Auslastung, günstiger als im Fall der in der linken Spalte dargestellten Alternative des Neukaufs. Vor diesem Hintergrund als auch wegen des verhältnismäßig kleinen Umfangs der Einsatzflächen in den ausgewählten Betrieben werden für weitere Kalkulationen vorbehaltlich des höheren Ausfallsrisikos die Kosten des Einsatzes einer Gebrauchtmaschine analysiert. 
Tabelle 6-11: Kostenkalkulation eines Silierwagens mit einem Ladevolumen von $26 \mathrm{~m}^{3}$ (ZL)

\begin{tabular}{|c|c|c|c|c|c|c|}
\hline Datengrundlagen & \multicolumn{3}{|c|}{ Neuer Silierwagen } & \multicolumn{3}{|c|}{ Gebrauchter Silierwagen (5 Jahre alt) } \\
\hline Anschaffungspreis & \multicolumn{3}{|c|}{140000} & \multicolumn{3}{|c|}{56000} \\
\hline Nutzungsdauer in $\mathrm{N} / \mathrm{t}$ & \multicolumn{3}{|c|}{ 12 Jahre/ $17000 \mathrm{t}$} & \multicolumn{3}{|c|}{6 Jahre/ $8500 \mathrm{t}$} \\
\hline Restwert & \multicolumn{3}{|c|}{14000} & \multicolumn{3}{|c|}{14000} \\
\hline \multicolumn{7}{|c|}{ Feste Kosten/ Jahr } \\
\hline AfA & \multicolumn{3}{|c|}{10500} & \multicolumn{3}{|c|}{7000} \\
\hline Zinsanspruch & \multicolumn{3}{|c|}{4620} & \multicolumn{3}{|c|}{2100} \\
\hline $\begin{array}{l}\text { Unterbringung und } \\
\text { Versicherung }\end{array}$ & \multicolumn{3}{|c|}{450} & \multicolumn{3}{|c|}{450} \\
\hline Summe feste Kosten & \multicolumn{3}{|c|}{15570} & \multicolumn{3}{|c|}{9550} \\
\hline \multicolumn{7}{|c|}{ Variable Kosten/ t } \\
\hline Reparaturen & \multicolumn{3}{|c|}{1,3} & \multicolumn{3}{|c|}{1,6} \\
\hline \multicolumn{7}{|c|}{ Gesamte Kosten } \\
\hline Einsatzumfang in $\mathrm{t}$ & $500 \mathrm{t}$ & $1000 \mathrm{t}$ & $2200 t$ & $500 \mathrm{t}$ & $1000 \mathrm{t}$ & $1500 \mathrm{t}$ \\
\hline Feste Kosten pro $t$ & 31 & 14 & 7 & 19 & 10 & 6 \\
\hline Variable Kosten pro t & \multicolumn{3}{|c|}{1,3} & \multicolumn{3}{|c|}{1,6} \\
\hline Summe pro $t$ & 32,3 & 17,3 & 8,3 & 21 & 11,6 & 7,6 \\
\hline Summe pro ha ${ }^{33}$ & 194 & 104 & 50 & 126 & 70 & 46 \\
\hline
\end{tabular}

Quelle: eigene Darstellung

In der Tabelle 6-12 werden zunächst die Anschaffungskosten des Silierwagens entsprechend den Anteilen der Einsatzflächen in den Mitgliedsbetrieben aufgeteilt. Der untere Teil dieser Tabelle enthält die unter den einzelnen Betrieben verteilten Kosten des Einsatzes des Silierwagens. Dabei wird die Annahme getroffen, dass auf dem Grünland zwei Schnitte pro Jahr erfolgen. Der durchschnittliche Ertrag der Anwelksilage pro ha liegt bei 6 Tonnen und der Arbeitszeitbedarf pro ha beträgt $0,5 \mathrm{~h}$.

Tabelle 6-12: Verteilung der Anschaffungs- und Einsatzkosten eines gemeinsamen gebrauchten Silierwagens nach Einsatzumfang (ZL)

\begin{tabular}{|c|c|c|c|c|c|}
\hline \multicolumn{2}{|c|}{ Beteiligter Landwirt } & \multicolumn{2}{|c|}{ Einsatzfläche/Arbeitszeit } & \multicolumn{2}{|c|}{ Finanzierungsbeitrag in ZL } \\
\hline \multicolumn{2}{|c|}{$\mathrm{I}$} & \multicolumn{2}{|c|}{$15 \mathrm{ha} / 15 \mathrm{~h}$} & \multicolumn{2}{|c|}{12880} \\
\hline \multicolumn{2}{|c|}{ II } & \multicolumn{2}{|c|}{$20 \mathrm{ha} / 20 \mathrm{~h}$} & \multicolumn{2}{|c|}{17360} \\
\hline \multicolumn{2}{|c|}{ III } & \multicolumn{2}{|c|}{$30 \mathrm{ha} / 30 \mathrm{~h}$} & \multicolumn{2}{|c|}{25760} \\
\hline \multicolumn{2}{|c|}{ Gesamt } & \multicolumn{2}{|c|}{$65 \mathrm{ha} / 65 \mathrm{~h}$} & \multicolumn{2}{|c|}{56000} \\
\hline \multirow[b]{4}{*}{$\begin{array}{c}\text { I } \\
\text { II } \\
\text { III }\end{array}$} & fest & sten pro Jahr & varial & 65 ha & Gesamte Kosten pro ha \\
\hline & & 9550 & & & 166 \\
\hline & \multicolumn{5}{|c|}{ Gesamte Kosten pro Betrieb } \\
\hline & \multicolumn{5}{|c|}{$\begin{aligned} 2196,5+287 & =2483,5 \\
2960,5+387 & =3347,5 \\
4393+574 & =4967\end{aligned}$} \\
\hline
\end{tabular}

In einem weiteren Schnitt werden die Kosten des Einsatzes des Silierwagens in der Maschinengemeinschaft mit der Alternative der Inanspruchnahme der Dienstleistung zu einem Preis von $160 \mathrm{ZL} /$ ha (320 ZL für zwei Schnitte) verglichen. Hinzu sind neben den

\footnotetext{
${ }^{33}$ Es wird hier vereinfacht angenommen, dass pro 1 ha Grünland in einem Schnitt $6 \mathrm{t}$ Anwelksilage geerntet werden.
} 
Kosten des Silierwagens auch die Kosten des Schleppers und des Bedienungspersonals zu berücksichtigen. Für den Vergleich werden die Kosten des gebrauchten Schleppers aus der Tabelle 6-2 herangezogen. Der Lohnansatz liegt wie in den früheren Beispielen bei $18 \mathrm{ZL} / \mathrm{h}$. Bei diesen Annahmen, ist der Einsatz des gebrauchten Silierwagens erst ab einem jährlichen Einsatzumfang von 44 ha günstiger als die Inanspruchnahme der Fremdmaschinenleistung. Bei dem neuen Silierwagen verschiebt sich die Mindestauslastungsschwelle auf 70 ha pro Jahr (siehe Abbildung 6-3).

Abbildung 6-3: Kosten des Einsatzes eines neuen und gebrauchten Silierwagens in Abhängigkeit vom Leistungsumfang $(\mathrm{ZL})^{34}$

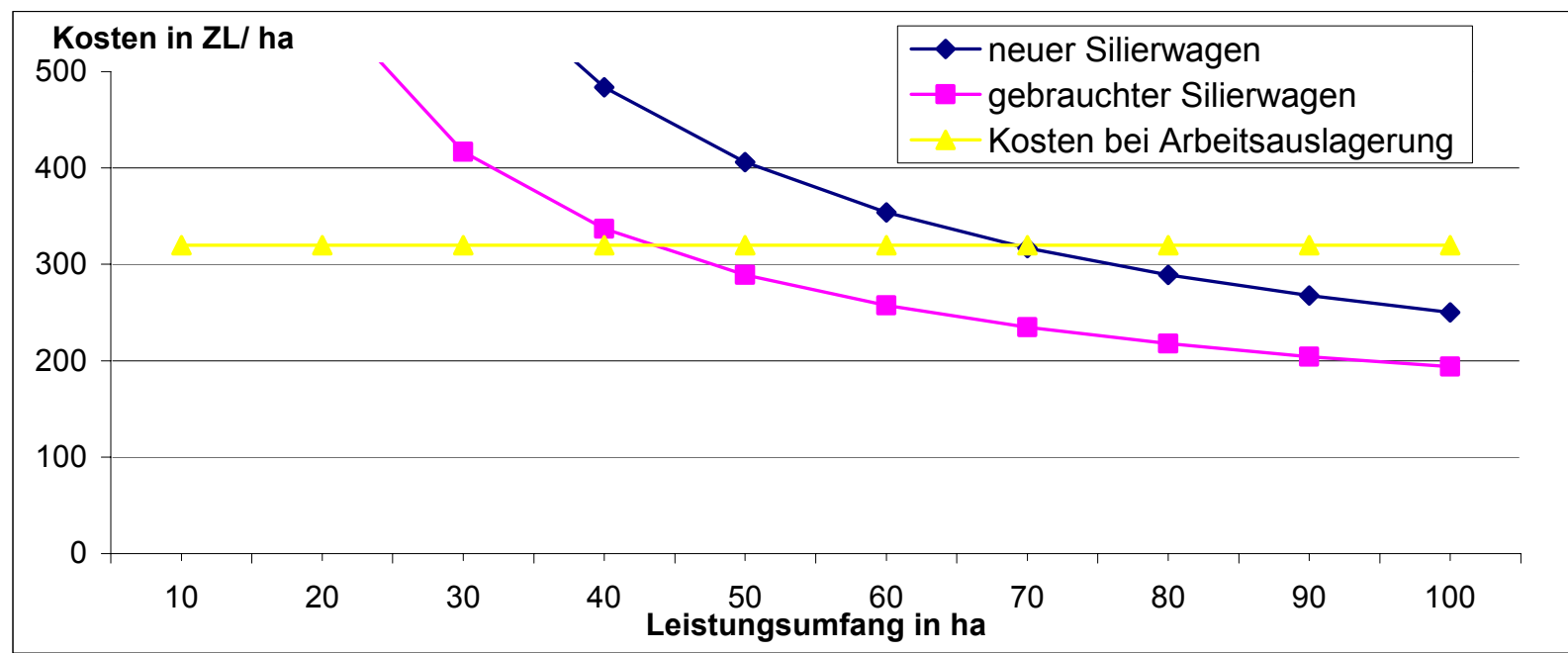

Quelle: eigene Darstellung

\section{Zuckerrübenbunkerköpfroder}

Ausgehend von einer Kooperationsbereitschaft im Bereich der Zuckerrübenernte werden die Kosten des Einsatzes eines dreireihigen Rübenbunkerköpfroders analysiert. Dazu werden zunächst in der Tabelle 6-13 die Kosten für zwei Varianten- einer neuen und einer gebrauchten Maschine- zusammengestellt.

\footnotetext{
${ }^{34}$ Gilt ebenso wie die vorherige Kalkulation für die Annahme, dass auf dem Grünland zwei Schnitte pro Jahr erfolgen.
} 
Tabelle 6-13: Kostenkalkulation eines dreireihigen Rübenbunkerköpfroders (ZL)

\begin{tabular}{|c|c|c|c|c|c|}
\hline \multirow{2}{*}{\begin{tabular}{|l|} 
Datengrundlagen \\
Anschaffungspreis
\end{tabular}} & \multicolumn{2}{|c|}{ Neuer Rübenbunkerköpfroder } & \multicolumn{3}{|c|}{$\begin{array}{l}\text { Gebrauchter Rübenbunkerköpfroder } \\
\text { (5 Jahre alt) }\end{array}$} \\
\hline & \multicolumn{2}{|c|}{180000} & \multicolumn{3}{|c|}{50000} \\
\hline Nutzungsdauer N/n & \multicolumn{2}{|c|}{ 10Jahre/ 1000 ha } & \multicolumn{3}{|c|}{$5 / 500$ ha } \\
\hline Restwert & \multicolumn{2}{|c|}{18000} & \multicolumn{3}{|c|}{18000} \\
\hline \multicolumn{6}{|c|}{ Feste Kosten/ Jahr } \\
\hline AfA & \multicolumn{2}{|c|}{16200} & \multicolumn{3}{|c|}{6400} \\
\hline Zinsanspruch & \multicolumn{2}{|l|}{5940} & \multicolumn{3}{|c|}{2040} \\
\hline $\begin{array}{l}\text { Unterbringung und } \\
\text { Versicherung }\end{array}$ & \multicolumn{2}{|l|}{300} & \multicolumn{3}{|c|}{300} \\
\hline Summe feste Kosten & \multicolumn{2}{|c|}{22440} & \multicolumn{3}{|c|}{8740} \\
\hline \multicolumn{6}{|c|}{ Variable Kosten/ ha } \\
\hline Reparaturen & \multicolumn{2}{|l|}{133} & \multicolumn{3}{|c|}{157} \\
\hline \multicolumn{6}{|c|}{ Gesamte Kosten } \\
\hline Einsatzumfang in ha & 30 & 120 & 30 & 60 & 90 \\
\hline Feste Kosten pro ha & 374 & 187 & 291 & 146 & 97 \\
\hline Variable Kosten pro ha & \multicolumn{2}{|l|}{133} & \multicolumn{3}{|c|}{157} \\
\hline Summe pro ha & 507 & 320 & 448 & 303 & 254 \\
\hline Summe pro $\mathrm{h}^{35}$ & 149 & 91 & 128 & 87 & 73 \\
\hline
\end{tabular}

Quelle: eigene Darstellung

Die Tabelle 6-14 enthält die Kosten des Einsatzes der Maschine in einer Maschinengemeinschaft von vier Landwirten. In Anlehnung an die KTBL- Daten wurde hier ein Arbeitszeitbedarf von 3,5 h pro ha unterstellt. Die weitere Vorgehensweise ist identisch wie in den früheren Beispielen.

Tabelle 6-14: Verteilung der Anschaffungs- und Einsatzkosten eines gemeinsamen Rübenbunkerköpfroders nach dem Einsatzumfang (ZL)

\begin{tabular}{|c|c|c|c|c|c|}
\hline \multicolumn{2}{|c|}{ Beteiligter Landwirt } & \multicolumn{2}{|c|}{ Einsatzfläche/Arbeitszeit } & \multicolumn{2}{|c|}{ Finanzierungsbeitrag in $\mathrm{ZL}$} \\
\hline \multicolumn{2}{|l|}{ I } & \multicolumn{2}{|c|}{$5 \mathrm{ha} / 17,5 \mathrm{~h}$} & \multicolumn{2}{|c|}{11000} \\
\hline \multicolumn{2}{|l|}{ II } & \multicolumn{2}{|c|}{$5 \mathrm{ha} / 17,5 \mathrm{~h}$} & \multicolumn{2}{|c|}{11000} \\
\hline \multicolumn{2}{|l|}{ III } & \multicolumn{2}{|c|}{$6 \mathrm{ha} / 21 \mathrm{~h}$} & \multicolumn{2}{|c|}{13000} \\
\hline \multicolumn{2}{|l|}{ IV } & \multicolumn{2}{|c|}{$7 \mathrm{ha} / 24,5 \mathrm{~h}$} & \multicolumn{2}{|c|}{15000} \\
\hline \multicolumn{2}{|c|}{ Gesamt } & $23 \mathrm{~h}$ & $30,5 \mathrm{~h}$ & \multicolumn{2}{|c|}{50000} \\
\hline \multirow[b]{4}{*}{$\begin{array}{c}\text { I } \\
\text { II } \\
\text { III } \\
\text { IV }\end{array}$} & \multicolumn{2}{|c|}{ feste Kosten pro Jahr } & \multicolumn{2}{|c|}{ variable Kosten pro 23 ha oder $80,5 \mathrm{~h}$} & Gesamte Kosten pro ha \\
\hline & & 40 & & 3611 & 537 \\
\hline & \multicolumn{5}{|c|}{ Gesamte Kosten pro Betrieb } \\
\hline & \multicolumn{5}{|c|}{$\begin{array}{l}1923+785=2708 \\
1923+785=2708 \\
2272+942=3214 \\
2622+1099=3721\end{array}$} \\
\hline
\end{tabular}

Quelle: eigene Darstellung

Für den Vergleich der Kosten der Mechanisierung in der Maschinengemeinschaft mit der Alternative der Arbeitsauslagerung zu einem Preis von $840 \mathrm{ZL} / \mathrm{ha}$ sind bei der Maschinengemeinschaft die Kosten des Schleppers und des Bedienungspersonals zu berücksichtigen. Werden hierzu dieselben Annahmen getroffen wie im vorangegangenen

\footnotetext{
${ }^{35}$ Es wird hier vereinfacht angenommen, dass die Leistung des Zuckerrübenbunkerköpfroders bei einer Schlaggröße von 1 ha einem ha pro 3,5 h entspricht.
} 
Beispiel des Silierwagens, so zeigt sich, dass der Einsatz des gemeinsamen gebrauchten Zuckerrübenbunkerköpfroders ab einer jährlichen Auslastung von 22 ha günstiger ist als die Inanspruchnahme der Fremdmaschinenleistung. Die Mindestauslastungsgrenze verschiebt sich bei der neuen Maschine auf 52 ha.

Abbildung 6-4: Kosten des Einsatzes des neuen und gebrauchten

Zuckerrübenbunkerköpfroders in Abhängigkeit vom Leistungsumfang (ZL)

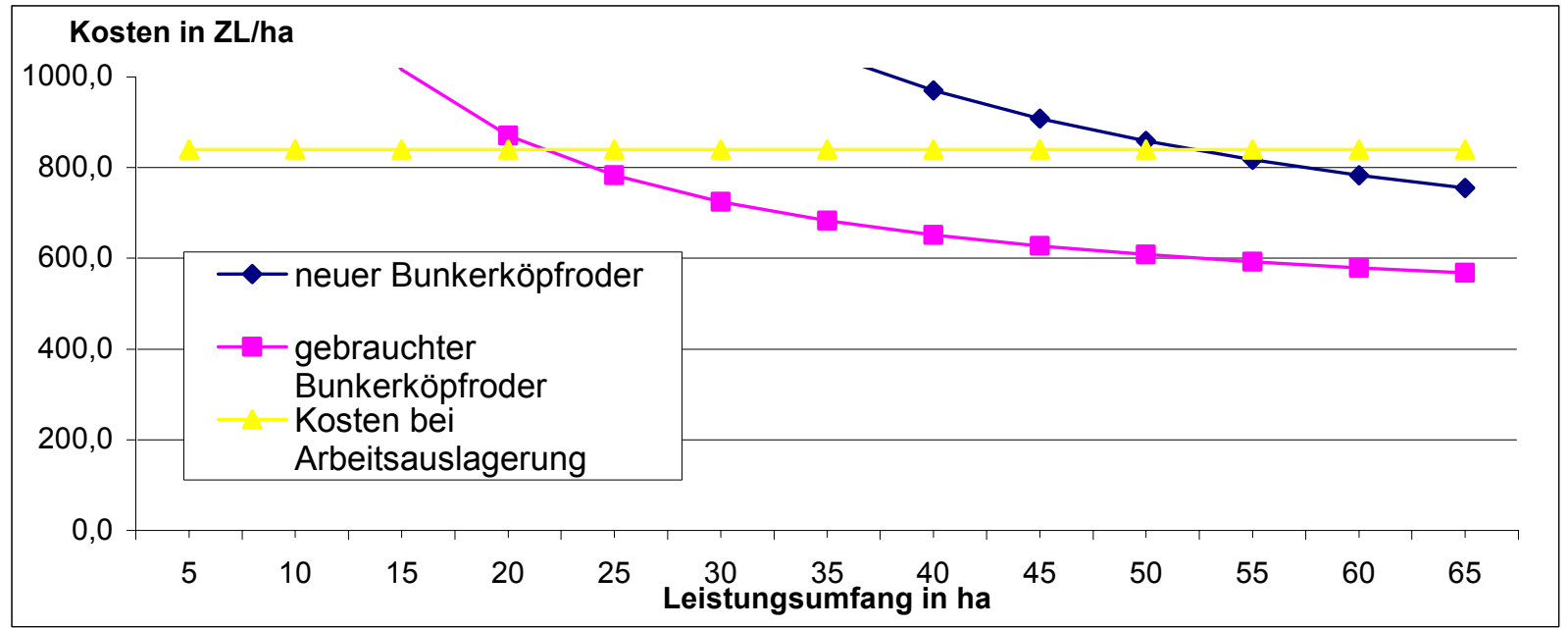

Quelle: eigene Darstellung

\subsection{Kritische Beurteilung}

Der Praxisbezug des dargestellten Konzepts der Modernisierung der Mechanisierung unter Berücksichtigung des Kooperationspotenzials ist von einer möglichst realen Einschätzung der Rahmenbedingungen beider Untersuchungsregionen und der daraus resultierenden Entwicklungsmöglichkeiten der landwirtschaftlichen Betriebe abhängig. Die Absicherung der herausgearbeiteten Vorschläge bedarf einer kritischen Beurteilung von der Seite der beteiligten Akteure vor Ort. Zu diesem Zweck war es notwendig erneut auf das Fachwissen von Beratern und anderen Angestellten der Institutionen des landwirtschaftlichen Umfelds zurückzugreifen. In einem ausgewählten Diskussionsforum wurden unter einer gleichzeitigen Berücksichtigung der wirtschaftlichen und sozialen Faktoren die in diesem Kapitel dargestellten Überlegungen kritisch beurteilt. Im Folgenden werden die diesbezüglichen Ergebnisse kurz erörtert. Um sie auf einer einheitlichen Basis darstellen zu können, wird hierzu auf die Ansätze eines für die Evaluierung des sozioökonomischen Umweltmanagements von SCHAltegger (1999, S. 12-14) entwickelten Konzepts zurückgegriffen. Dieses Konzept wird für die Überlegungen dieser Arbeit modifiziert und weiterhin als Grundgerüst zur Beurteilung der ausgewählten Kooperationsformen herangezogen. Hierzu werden folgende Kriterien angewendet. 


\section{Die Akzeptanz.}

Da die dargestellten Modifizierungsschritte nur freiwillig bei Engagement der Landwirte vollbracht werden können und die diesbezüglichen Vorteile betriebsindividuell zu gestalten sind, muss die Akzeptanz der Beteiligten für bestimmte Maßnahmen untersucht werden.

\section{Die Effizienz.}

Dieses Kriterium dient als Maßstab für die Beurteilung des Aufwandes, der erforderlich ist, um konkrete Verbesserungen herbeizuführen.

\section{Die Integration.}

Es muss zunächst geprüft werden, inwieweit die Zusammenarbeit in ausgewählten Kooperationsformen mit der voraussichtlichen Betriebsentwicklung konform ist. Darüber hinaus sind hier die aktuellen wirtschaftlichen und agrarpolitischen Aspekte der Umsetzung der Verbesserungsvorschläge zu bedenken.

\section{Die Innovation.}

Das Ziel der formulierten Verbesserungsvorschläge ist u.a., zur Beschleunigung des Maschinenwechsels und Einführung neuerer als bisherige Technik beizutragen. Mithilfe des Kriteriums der Innovation ist zu beurteilen, inwieweit die ausgewählten Kooperationsformen dieser Voraussetzung Rechnung zu tragen vermögen. Der Aspekt der Innovation ist an dieser Stelle im Zusammenhang mit den Kriterien Akzeptanz und Effizienz der vorgeschlagenen Maßnahmen zu erwägen.

Die Übersicht 6-1 zeigt einen einfachen Bewertungsansatz mit den bereits geschilderten Bewertungskriterien. Da die Aussagen der befragten Experten zu Entwicklungsmöglichkeiten der MR weitgehend mit den früher formulierten Ansatzpunkten übereinstimmen, wird diese Form der Zusammenarbeit in die Beurteilung nicht einbezogen.

Übersicht 6-1: Bewertungskriterien zur Analyse der Entwicklungschancen ausgewählter Kooperationsformen

\begin{tabular}{|c|c|c|c|c|}
\hline & Akzeptanz & Effizienz & Integration & Innovation \\
\hline Nachbarschaftshilfe & hoch & mittel & mittel & niedrig \\
\hline Kleine Maschinengemeinschaft & mittel & hoch & hoch & hoch \\
\hline Große Maschinengemeinschaft & niedrig & hoch & mittel & hoch \\
\hline LU & niedrig & mittel & niedrig & mittel \\
\hline
\end{tabular}

Quelle: eigene Erhebung

Wie an früheren Stellen angesprochen, besitzt eine mit deutlichem Abstand höchste Akzeptanz in beiden Untersuchungsregionen die informelle Nachbarschaftshilfe. Dabei wird von den Landwirten v.a. die Flexibilität und Unabhängigkeit geschätzt. Die Beurteilung der 
Effizienz dieser Form der Zusammenarbeit setzt eine Differenzierung der Gruppe der untersuchten Betriebe voraus. Während in kleineren Betrieben die gegenseitige Nachbarschaftshilfe bei einem relativ niedrigen Aufwand zur Optimierung der Mechanisierung beitragen kann, ist sie in größeren Betrieben, besonders bei komplizierten Verfahren, nicht in der Lage auf die Organisation der Maschinenarbeit und im weiteren Sinn auf die Betriebsentwicklung fördernd zu wirken. Vor diesem Hintergrund ist diese Form der Zusammenarbeit auch in Bezug auf das Kriterium der Integration zu beurteilen. Unter Berücksichtigung weiterer Entwicklung der Betriebe beider Regionen soll die Nachbarschaftshilfe, abgesehen von kleineren nicht wachstumsfähigen Betrieben, kontinuierlich zugunsten von bindungsintensiveren Kooperationsformen oder Eigenmechanisierung mit teilweiser außerbetrieblicher Arbeitserledigung an Bedeutung verlieren.

Es werden von den befragten Experten die Entwicklungschancen der kleinen Maschinengemeinschaften als hoch eingestuft. Dies betrifft in erster Linie die Zusammenschlüsse für die gemeinsame Anschaffung von Mähdreschern. Unter Berücksichtigung der Ergebnisse der primären Befragung, die den Zustand der Mähdrescher zeigten, wird diese Kooperationsform als ein wesentliches Instrument der Modernisierung der Mechanisierung des Mähdrusches anerkannt. Angesicht der verhältnismäßig niedrigen Kosten der bisherigen Mechanisierung ist es angebracht, bei Beurteilung dieser Kooperationsform die Kriterien Effizienz und Innovation miteinander zu verbinden. Es wurde angemerkt, dass die Maschinenersatzinvestitionen $\mathrm{zu}$ den in den vorangegangenen Kalkulationen unterstellten Anschaffungspreisen in einzelbetrieblicher Lösung in meisten Fällen schwierig realisierbar sind. Durch die Zusammenarbeit in Maschinengemeinschaften kann bei einem reduzierten Aufwand ein spürbarer Innovationseffekt herbeigeführt werden. Als positiv und der realen Investitionsfähigkeit der landwirtschaftlichen Betriebe angepasst, wird die analysierte Alternative der Anschaffung der gebrauchten Maschinen bewertet. Ebenso positiv wird der Integrationsgrad dieser Form der Zusammenarbeit in die voraussichtliche Entwicklung der landwirtschaftlichen Betriebe beider Regionen beurteilt. Es handelt sich hier zum einen um die Mechanisierungsvorteile in den miteinander agierenden mittleren und größeren Betrieben, zum anderen um die potenzielle Auswirkung der Maschinengemeinschaften auf das Angebot an Fremdmaschinenleistung. Als ein wesentliches Hindernis für eine schnelle Verbreitung der Maschinengemeinschaften wird die noch bestehende starke Abneigung gegen gemeinsames Maschineneigentum hervorgehoben. 
Die dargestellten Ausführungen gelten in demselben Maße für den analysierten Zusammenschluss von drei Landwirten für die Anschaffung und den anschließenden Einsatz eines Silierwagens.

Demgegenüber sind die Experten skeptisch in Bezug auf die Entwicklungschancen der Maschinengemeinschaft mit einem dreireihigen Zuckerrübenbunkerköpfroder. Dabei handelt es sich nicht um die Akzeptanz oder Effizienz, die nicht niedriger als in anderen Beispielen der Maschinengemeinschaft eingeschätzt werden, sondern vielmehr um die Kriterien der Integration und Innovation. Nach Aussagen der Experten erfolgt die Zuckerrübenernte in der Mehrheit der Betriebe durch Anwendung von zweireihigen Zuckerrübenbunkerköpfroder. In einigen wenigen Betrieben werden in diesem Verfahren moderne Maschinen eingesetzt, deren Auslastung durch einen entgeltlichen Einsatz in anderen Betrieben gesteigert wird. In den kleineren Betrieben werden Ersatzinvestitionen zzt. nicht vorgenommen und sind auch nicht geplant. Die Änderung der Mechanisierungsstruktur wird, nach Einschätzung der befragten Experten, durch Verzicht auf eigene Maschinen und Auslagerung der Zuckerrübenernte an Betriebe mit moderner schlagkräftiger Ausstattung erfolgen. Vor diesem Hintergrund wird die vorher analysierte Gemeinschaft von vier Landwirten mit einem dreireihigen Zuckerrübenbunkerköpfroder als eine kurzfristige Lösung betrachtet, die angesichts des erforderlichen Investitionsaufwands nur für eine eingeschränkte Gruppe der landwirtschaftlichen Betriebe geeignet erscheint. In diesem Zusammenhang rückt in Vordergrund entweder der Lohnmaschineneinsatz bis zu professioneller Dienstleistung oder eine größere Gemeinschaft, die von einer neutralen Institution geführt wäre. In diesem konkreten Fall könnte also der Betrachtungszeitraum verlängert und könnten die beispielhaften Kalkulationen um die Kosten des Einsatzes eines modernen Zuckerrübenroders in einer Gemeinschaft mehrerer Landwirte erweitert werden.

Bis auf die bereits genannte Lohnmaschinenleistung bei der Zuckerrübenernte und in einem eingeschränkten Maße die vorher angesprochene Organisation der Silierkette, werden von den befragten Experten die Entwicklungschancen der professionellen $L U$ negativ beurteilt. Als Hauptbegrenzungsfaktor hierfür wird die niedrige Ertragsfähigkeit der landwirtschaftlichen Betriebe und das Fehlen von Alternativen für die Anwendung der vorhandenen AK und der Maschinenkapazitäten genannt. Im Hinblick auf die früher angesprochenen Bereiche des Mähdrusches und des Pflanzenschutzes wird die Entwicklung der professionellen Dienstleistung v.a. wegen der Termingerechtheit angezweifelt. Dem Kriterium Innovation und den daraus resultierenden positiven Effekten auf die Betriebsentwicklung wird von den 
befragten Experten beider Regionen kaum Bedeutung beigemessen. Als wahrscheinlich für die nächsten Jahre wird nur eine weitere Entwicklung des Angebots der Lohnmaschinenleistung von den größeren Betrieben mit freien Maschinenkapazitäten bezeichnet.

\subsection{Zusammenfassung}

Mit der überbetrieblichen Zusammenarbeit kann in den Untersuchungsregionen, abgesehen von einzelnen Betrieben, nicht das Ziel verwirklicht werden, die Mechanisierungskosten zu senken. Mithilfe der vorhandenen Maschinen mit einer zusätzlichen Inanspruchnahme der Fremdmaschinenleistung, die im Wesentlichen im Rahmen der unentgeltlichen Nachbarschaftshilfe erfolgt, werden zzt. die Maschinenarbeiten recht günstig erledigt. Die Modernisierung der Mechanisierung ist jedoch für die Verbesserung der Wettbewerbsfähigkeit, u.a. durch das Betriebswachstum dringend notwendig. Infolge einer niedrigen Kapitalausstattung und beschränkter Möglichkeiten der Auslastung neuer schlagkräftiger Technik stoßen die meisten Betriebe beider Stichproben ziemlich schnell an die Entwicklungsgrenzen. In beiden Untersuchungsregionen liegen aber, wenn auch in unterschiedlicher Ausprägung, günstige Bedingungen für die Entwicklung überbetrieblicher Zusammenarbeit vor.

In der Region Ermland-Masuren kann sich zunächst eine Zusammenarbeit zwischen Betrieben mit Konzentration auf die Milchkuhhaltung und größeren Ackerbaubetrieben entwickeln. In den erstgenannten Betrieben kann die partielle Auslagerung der Maschinenarbeiten einen fördernden Einfluss auf weiteres, zumeist arbeits- und kapitalaufwendiges, Wachstum im Innenbereich haben. Im Fall der größeren Betriebe führt die Möglichkeit des Einsatzes der vorhandenen Maschinen in anderen Betrieben zu einer Auslastungssteigerung damit zur Senkung der Kosten je Produktionseinheit.

Als geeignet für diese Region erscheinen kleinere Maschinengemeinschaften. Dies betrifft sowohl die mittleren als auch die größeren Betriebe in denen die Anwendung moderner Technik in einzelbetrieblicher Lösung v.a. aus finanziellen Gründen aber auch wegen der bereits angesprochenen eingeschränkten Möglichkeiten der Sicherung der erforderlichen Kapazitätenauslastung der schlagkräftigen Maschinen wirtschaftlich nicht realisierbar wäre.

Eine wesentliche Bedeutung in der weiteren Entwicklung der Mechanisierung dieser Region kann ein zunehmend in Form der professionellen Dienstleistung erfolgender 
Lohnmaschineneinsatz haben, der vorwiegend von den Landwirten selbst organisiert wird. Die Nachfrage nach Dienstleistungen kann künftig sowohl von den Betrieben der Tierhaltung als auch von jenen Betrieben kommen, die weiterhin zwar als selbständige Einheiten jedoch ohne weiteres Wachstum z.B. im Nebenerwerb geführt werden.

In der Region Lublin kann die überbetriebliche Zusammenarbeit einen wesentlichen Beitrag zur Minderung des Problems der ungünstigen Betriebsgrößenstrukturen leisten. Besonders zu begrüßen wäre hier die Entwicklung von kleinen Maschinengemeinschaften in mittleren und größeren Betrieben. Es kann hier allmählich auch die Nachfrage nach der Fremdmaschinenleistung weiter ansteigen, denn dadurch können wesentliche betriebsstrukturelle Vereinfachungen vorgenommen werden. Dies ist besonders in den kleineren Betrieben wichtig, wo der Fremdmaschineneinsatz auf eine Änderung der AKAusstattung und eine notwendige Verlagerung des Schwerpunktes der vorhandenen Kapazitäten auf außerlandwirtschaftliche Tätigkeiten fördernd wirken kann. Es werden hier die Voraussetzungen geschaffen, die zugänglichen Faktoren effizient und wachstumsorientiert anzuwenden. Darüber hinaus kann das Angebot an Lohmaschinenleistungen, v.a. in den kleineren und mittleren Betrieben, $\mathrm{zu}$ einem neuen Betriebszweig mit positiven Einkommenseffekten ausgebaut werden. Dies gilt neben dem Einsatz der Maschinen in anderen Betrieben auch für die Dienstleistung im kommunalen Bereich (z.B. die Pflege der Straßenränder und der Graben, der Winterdienst, die Abfallabfuhr) oder auch für die Entwicklung der Gewerbetätigkeit im Transportbereich.

Die Auswahl der optimalen Mechanisierungsform kann nur betriebsindividuell vor dem Hintergrund weiterer Entwicklungsentscheidungen erfolgen. Es ist dabei sorgfältig zu prüfen, welche Einspareffekte bestimmte Mechanisierungsformen einschließlich Inanspruchnahme der Fremdmaschinenleistung bieten. Die dargestellten Kalkulationen können als eine Orientierungshilfe für die Auswahl einer zweckmäßigen Mechanisierungsform dienen.

Bei den Ersatzinvestitionen ist es wichtig, dass die neuen Maschinen dem betrieblichen Leistungsbedarf und der etwaigen Nachfrage nach Maschinenarbeit in der Region angepasst werden. In diese Überlegungen ist die Anschaffung gebrauchter Maschinen einzubeziehen. Dies betrifft besonders die Betriebe, die angesichts fehlender Alternativen für die Anwendung vorhandener Produktionsfaktoren entweder auf eine weitere Eigenmechanisierung oder auf eine Zusammenarbeit in kleineren Gemeinschaften abzielen. Es können dadurch die Mechanisierungskosten im Fall der Modernisierung deutlich gesenkt werden. 
Die Anschaffung von neuen schlagkräftigen Maschinen ist besonders für die Betriebe zu empfehlen, die künftig den Lohnmaschineneinsatz als einen zusätzlichen Betriebszweig auszubauen beabsichtigen. Denn entscheidend hierbei ist neben der Leistungsfähigkeit und der Qualität auch der Aspekt der Zuverlässigkeit und Termingerechtheit der verrichteten Arbeiten. 


\section{Formen der Investitionsfinanzierung und Ermittlung der optimalen Ersatzstrategie der Maschinen}

Im Folgenden werden zunächst Überlegungen $\mathrm{zu}$ Finanzierungsformen der Maschineninvestitionen vorgenommen. Dabei erfolgt eine allgemeine Charakteristik des diesbezüglichen Förderungsprogramms des MRiRW. Weiterhin wird die Finanzierung mittels zinsverbilligter und herkömmlicher Kredite anhand beispielhafter Tilgungspläne verglichen. Als eine Alternative zur Finanzierung mittels Kredits wird hier auch das Leasing analysiert. In einem weiteren Schritt wird in Bezug auf die in Kapitel 6.4 dargestellten theoretischen Grundlagen der Kostenkalkulation des Maschineneinsatzes die Methodik zur Ermittlung der optimalen Ersatzstrategie am Beispiel eines Schleppers geschildert.

\subsection{Vorteilhaftigkeitsanalyse der Finanzierung mittels zinsverbilligter und nicht zinsverbilligter Kredite}

Im Rahmen der Investitionsförderung des polnischen MRiWR lassen sich zwei Instrumente unterscheiden. Es handelt sich hier zum einen um die Förderung in Form verlorener Zuschüsse, die im Rahmen des sog. Sektoralen Operativen Programms (SOP) erfolgt und zum anderen um die Gewährung von Zinsverbilligung für Kapitalmarktdarlehen im Branchenkreditprogramm.

Im SOP können $50 \%$ bis $65 \%$ der Kosten bestimmter Investitionen zurückerstattet werden. Die Förderung kann nur den Betrieben gewährt werden, die die Voraussetzung der Existenzsicherheit erfüllen. Als Maßstab zur Beurteilung der Existenzsicherheit dient die anhand von SDB ermittelte EGE der Betriebe ${ }^{36}$. In Genuss der Fördermittel können Betriebe kommen, die eine Größe von mindestens 4 EGE aufweisen. Diese Anordnung kann unter bestimmten Bedingungen modifiziert werden, so dass die Fördermittel auch von Betrieben in Anspruch genommen werden können, die zwar die Voraussetzung der betriebswirtschaftlichen Größe zum Zeitpunkt der Antragstellung nicht erfüllen, jedoch auf das Erreichen des erforderlichen Niveaus von 4 EGE spätestens in 5 Jahren nach der Tätigung der Investition schließen lassen ${ }^{37}$.

\footnotetext{
36 Es werden hierzu vier Gebiete unterschieden innerhalb von denen die SDB regionalisiert für 34 Produktionszweige der Bodennutzung und 18 Tierhaltungsmerkmale berechnet werden.

37 Für die Interpretation dieser Größe vor dem Hintergrund des westlichen Standards wird auf die Betriebsklassifizierung in der EU verwiesen, wo landwirtschaftliche Betriebe von 8 bis 16 EGE als Klein- und Nebenerwerbsbetriebe gelten.
} 
Im Branchenkreditprogramm, das in verschiedene Kreditlinien gegliedert ist, werden von bestimmten Banken Kredite mit einem jährlichen nominellen Zinssatz von 1,20 \% bis 3,57 \% angeboten $^{38}$. Einen Überblick über die Kredite, die unter anderem für die Finanzierung der Maschineninvestitionen in Anspruch genommen werden können, gibt die Übersicht 7-1.

Übersicht 7-1: Kreditlinien für die Förderung der Maschineninvestitionen

\begin{tabular}{|c|c|c|c|}
\hline Kreditlinie & Jährlicher Zinssatz & Kreditvolumen & Kreditlaufzeit \\
\hline Grundinvestitionen (IP) & bis 3,09\% & $\begin{array}{c}\text { max. 80\% des } \\
\text { Investitionsaufwands } \\
\text { nicht mehr als 8 Mio. ZL }\end{array}$ & $\begin{array}{c}\text { max. 8 Jahre mit einer } \\
\text { tilgungsfreien Zeit von 2 } \\
\text { Jahren }\end{array}$ \\
\hline Brachenkredite (BR) & bis 2,00\% & $\begin{array}{c}\text { max. 80\% des } \\
\text { Investitionsaufwands } \\
\text { nicht mehr als 8 Mio. ZL }\end{array}$ & $\begin{array}{c}\text { max. 8 Jahre mit einer } \\
\text { tilgungsfreien } \\
\text { Zeit von 3 Jahren }\end{array}$ \\
\hline $\begin{array}{c}\text { Kredite für junge } \\
\text { Landwirte }\end{array}$ & bis 1,20\% & $\begin{array}{c}\text { max. 80\% des } \\
\text { Investitionsaufwands } \\
\text { nicht mehr als 8 Mio. ZL }\end{array}$ & $\begin{array}{c}\text { max. 15 Jahre mit einer } \\
\text { tilgungsfreien } \\
\text { Zeit von 2 Jahren }\end{array}$ \\
\hline
\end{tabular}

Quelle: MRiRW 2005

Die Förderungsfunktion des MRiRW im Mechanisierungsbereich kann anhand des seit dem Jahr 1997 bestehenden Branchenprogramms BR/10 für die gemeinsame Nutzung von landwirtschaftlichen Maschinen und Geräten dargestellt werden. Die Adressaten des Programms sind zum einen Landwirte, die bestimmte Maschinen gemeinsam zu nutzen beabsichtigen, zum anderen Unternehmen der Lohnmaschinenleistung. Die gesamte Quote des Kredits kann max. 4 Mio. ZL bzw. 8 Mio. ZL für Betriebe der Spezialbereiche betragen ${ }^{39}$. Der erforderliche Anteil der Eigenmittel beträgt $20 \%$ in Betrieben der landwirtschaftlichen Produktion und $30 \%$ in Unternehmen der Lohmaschinenleistung. Der Kredit wird max. für 8 Jahre gewährt, wobei die tilgungsfreie Zeit nicht über 3 Jahre hinausgehen kann. Die Beantragung des Kredits setzt die Vorbereitung eines detaillierten Investitionsplanes voraus, der in einem ersten Schritt einer landwirtschaftlichen Beratungsstelle vor Ort vorzulegen ist. Der Investitionsplan kann nur im Fall von Betrieben anerkannt werden, die als entwicklungsfähig eingestuft werden. Die Untersuchung der Kreditwürdigkeit der Antragsteller erfolgt dabei nicht von ARiMR, sondern von den Banken, die die Kredite vergeben.

\footnotetext{
${ }^{38} \mathrm{Zu}$ der Berechnungsmethodik des Zuschusses ist Folgendes anzumerken. Es können von bestimmten Banken Kredite angeboten werden, deren jährlicher Zinssatz nicht über 1,3 des Rediskontsatzes liegt (zum 01.03.06 betrug der Rediskontsatz 4,25 \%). Der von MRiRW gewährte Zuschuss kann maximal 0,75 des Zinssatzes dieser Kredite betragen.

${ }^{39}$ Unter den Betrieben der Spezialbereiche werden die Betriebe der Spezialkulturen, die Veredlungsbetriebe und die Betriebe verstanden, in denen neue Arbeitsplätze geschaffen werden.
} 
Der Vergleich der Finanzierung mittels eines zinsverbilligten und nicht zinsverbilligten Kredits wird anhand des praktischen Beispiels eines Schleppers aus der vorangegangenen Kalkulation vorgestellt. Es wird davon ausgegangen, dass die Ertragslage der landwirtschaftlichen Betriebe nicht ausreichend gut ist, als dass die für die vollständige oder sogar überwiegende Selbstfinanzierung erforderlichen Überschüsse erwirtschaftet werden könnten. Das Eigenkapital wird in der Investitionsplanung nur in dem vom Kreditgeber vorausgesetzten Rahmen mit berücksichtigt. Dabei bleibt es offen und wird im Weiteren nicht erörtert, ob die erforderlichen Eigenmittel aus Desinvestitionen oder auf einem anderen Weg gewonnen werden. Für die Ermittlung der sich aus dem Kapitaldienst ergebenden Liquiditätsbelastung werden in der Tabelle 7-2 einfache Tilgungspläne erstellt. Es gelten hier die in oberen Teil der Tabelle dargestellten Annahmen. Aus Vereinfachungsgründen wird in dieser Kalkulation die tilgungsfreie Zeit nicht berücksichtigt. Da es sich in dem dargestellten Beispiel um ein Annuitätsdarlehen handelt, ist der Kapitaldienst während der gesamten Kreditlaufzeit konstant. Die jährliche Annuität ergibt sich aus der Multiplizierung des Nennbetrages des Kredites mit dem entsprechenden Wiedergewinnungsfaktor (WF ${ }_{i}$; $)$. Die Zinsen, die für jedes einzelne Jahr ausgewiesen worden sind, errechnen sich aus jeweiliger Restschuld. Die Tilgung wird als Differenz zwischen der jährlichen Annuität und den anfallenden Zinsen ermittelt. Im unteren Teil der Tabelle 7-1 wird analog ein Tilgungsplan für die Finanzierung bei Aufnahme eines herkömmlichen Kredits dargestellt. Bis auf den Zinssatz der in diesem Beispiel $6 \%$ beträgt, sind die hierzu getroffenen Annahmen identisch mit denen in der Kalkulation des zinsverbilligten Kredits. 
Tabelle 7-1: Beispielsrechnung für Investitionsfinanzierung eines Schleppers (ZL)

\begin{tabular}{|c|c|c|c|c|}
\hline \multicolumn{5}{|c|}{ Investitionsfinanzierung bei Inanspruchnahme eines verbilligten Kredits } \\
\hline \multicolumn{5}{|c|}{$\begin{array}{l}\text { Annahmen: } \\
-\mathrm{A}=140000 \mathrm{ZL} \\
\text { - Nennbetrag des Kredits }=112000 \mathrm{ZL} \\
\text { - EK }=20 \%, \mathrm{i}=2 \%\end{array}$} \\
\hline \multirow[t]{2}{*}{$\mathrm{Jahr}$} & \multirow[t]{2}{*}{ Restschuld } & \multicolumn{2}{|c|}{ Kapitaldienst } & \multirow[t]{2}{*}{ Annuität } \\
\hline & & Tilgung & Zinsen & \\
\hline 1 & 112000 & 13049 & 2240 & 15289 \\
\hline 2 & 98951 & 13310 & 1979 & 15289 \\
\hline 3 & 85641 & 13576 & 1713 & 15289 \\
\hline 4 & 72065 & 13848 & 1441 & 15289 \\
\hline 5 & 58217 & 14125 & 1164 & 15289 \\
\hline 6 & 44092 & 14407 & 882 & 15289 \\
\hline 7 & 29685 & 14695 & 594 & 15289 \\
\hline 8 & 14989 & 14989 & 300 & 15289 \\
\hline Summe & - & 112000 & 10313 & 122313 \\
\hline \multicolumn{5}{|c|}{ Investitionsfinanzierung bei Inanspruchnahme eines nicht verbilligten Kredits } \\
\hline \multicolumn{5}{|c|}{$\begin{array}{l}\text { Annahmen: } \\
\mathrm{i}=6 \%\end{array}$} \\
\hline \multirow[t]{2}{*}{ Jahr } & \multirow[t]{2}{*}{ Restschuld } & \multicolumn{2}{|c|}{ Kapitaldienst } & \multirow[t]{2}{*}{ Annuität } \\
\hline & & Tilgung & Zinsen & \\
\hline 1 & 112000 & 11316 & 6720 & 18036 \\
\hline 2 & 100684 & 11995 & 6041 & 18036 \\
\hline 3 & 88689 & 12715 & 5321 & 18036 \\
\hline 4 & 75974 & 13478 & 4558 & 18036 \\
\hline 5 & 62497 & 14286 & 3750 & 18036 \\
\hline 6 & 48211 & 15143 & 2893 & 18036 \\
\hline 7 & 33067 & 16052 & 1984 & 18036 \\
\hline 8 & 17015 & 17015 & 1021 & 18036 \\
\hline Summe & - & 112000 & 32288 & 144288 \\
\hline
\end{tabular}

Dieses Vergleichsbeispiel kann weiter durch die Ermittlung der effektiven Zinssätze $\left(Z_{\text {eff }}\right)$ unter Berücksichtigung aller Kosten die mit der Aufnahme und Zurückzahlung des Kredites verbunden sind, analysiert werden. Im Fall des verbilligten Kredits sind an dieser Stelle zusätzlich die Kosten der Vorbereitung eines Investitionsplans in Höhe von $1 \%$ des Nennbetrages und ein Disagio von $6 \%$ hinzuzurechnen. Bei dem nicht verbilligten Kredit wird auch mit einem Disagio von $6 \%$ und keinen zusätzlichen Bearbeitungskosten kalkuliert. Der effektive Zinssatz wird durch die Ermittlung eines entsprechenden Wiedergewinnungsfaktors (WF) anhand folgender Gleichung berechnet (vgl. BRANDES, ODENING 1992, S. 74 ff.):

$W F \mathrm{i}_{\mathrm{eff} ; \mathrm{N}}=\frac{W F i_{\text {nom }} ;_{N}}{1-\frac{x}{100}}$

Dabei bedeutet:

$\mathrm{x}$ - Disagio + Kosten der Vorbereitung des Investitionsplans. 
Der unter diesen Bedingungen ermittelte $Z_{\text {eff }}$ des verbilligten Kredites beträgt 3,7 \%. Bei dem nicht verbilligten Kredit liegt der $Z_{\text {eff }}$ bei 7,5 \%. Es kann nunmehr der Frage nachgegangen werden, wie groß der Beihilfeanteil des Kredites mit der Zinsverbilligung ist. Es wird dabei der WF des verbilligten Kredits durch den WF des unverbilligten Kredits dividiert (vgl. ebenda).

$$
x=\frac{W F_{\text {verbilligt }}}{W F_{\text {unverbilligt }}}
$$

Der gesuchte Faktor 1-x gibt den prozentualen Beihilfeanteil des verbilligten Kredits wieder. Im dargestellten Beispiel beträgt der Beihilfeanteil 14,3\%. Das bedeutet, dass der Umfang des nicht verbilligten Kredits um 14,3\% gesenkt werden müsste, damit der Kapitaldienst dieses Kredites dem verbilligten Kredit gleichen würde.

Im Anschluss kann bei der Untersuchung der Kapitalkosten (KK) beider Finanzierungsformen die relative Ersparnis des verbilligten Kredits ermittelt werden. Hierzu wird auf die in Kapitel 6.4.4 dargestellte Formel für die Ermittlung der durchschnittlichen Kosten der Maschinen zurückgegriffen. Unter Berücksichtigung des Anteils des Eigenkapitals (EK) und des Fremdkapitals (FK) können die Kapitalkosten der Investition anhand folgender Gleichungen berechnet werden.

Fall A. Für den verbilligten Kredit.

$$
K K_{\text {verbilligt }}=\frac{A-R W}{N}+(E K+R W) \times 0,5 \times i_{E K}+(F K+R W) \times 0,5 \times i_{F K \text { verbilligt }}
$$

Fall B. Für den nicht verbilligten Kredit.

$$
K K_{\text {unverbiligt }}=\frac{A-R W}{N}+(E K+R W) \times 0,5 \times i_{E K}+(F K+R W) \times 0,5 \times i_{F K u n v e r b i l l i g t}
$$

Bei der Zugrundelegung eines Kalkulationszinssatzes für das eingesetzte EK von $4 \%$ pro Jahr und der bereits ermittelten $Z_{\text {eff }}$ beider Finanzierungsformen sind die KK des verbilligten Kredits um 2.394 ZL, d.h. 17,14 \% niedriger als die des herkömmlichen Kredits.

\subsection{Leasing als Alternative zum Kauf}

Unter Leasingvertrag wird i.e.S. ein Vertrag verstanden, anhand dessen ein Leasinggeber einen Gegenstand einem Leasingnehmer für einen festgelegten Zeitraum gegen ein bestimmtes Entgelt zur Verfügung stellt. In der Praxis wird zwischen zwei Leasingformen unterschieden. Die erste Form bildet das sog. Operate-Leasing, die zweite demgegenüber das Finanzierungs-Leasing. Das Hauptkriterium der Unterscheidung beider Formen sind die 
Eigentumsrechte bezüglich des geleasten Gegenstandes. Im Fall von Operate-Leasing handelt es sich um einen kurzfristigen Mietvertrag, anhand dessen der geleaste Gegenstand nach dem Ablauf der Vertragsfrist zum Leasinggeber zurückkehrt. In diesem Vertrag wird dem Leasingnehmer i.d.R. ein Kündigungsrecht eingeräumt. Unter dem Begriff FinanzierungsLeasing werden mittel- und langfristige Verträge verstanden, in denen eine Übernahme des Leasinggegenstandes durch den Leasingnehmer nach Ablauf der Vertragsfrist vorgesehen ist. Die Verträge in dieser Form sind meist unkündbar (vgl. SPITTLER 2002, S. 21 ff.).

Leasing als eine Vertragsform wurde im polnischen Rechtsystem erst im Jahr 2000 in einem Änderungsgesetz des Zivilrechtes expliziert kodifiziert (vgl. Dz.U.00.74.857). Grundlegend für die steuerliche Behandlung von Leasing sind die entsprechenden Steuergesetze aus dem Jahr 2001 (vgl. Dz.U.01.106.1150). Im Sinne dieser Schreiben handelt es sich um ein Operations-Leasing bei Verträgen, die für eine konkrete Zeit (zumindest $40 \%$ der AfADauer) abgeschlossen werden und deren Summe, der in ihnen festgelegten Raten mindestens die Anschaffungs- oder Herstellungskosten abzüglich der Umsatzsteuer deckt. Der Leasinggegenstand ist in diesem Fall dem Leasinggeber zuzurechnen, d.h. der Leasinggeber hat den geleasten Gegenstand mit Anschaffungs- oder Herstellungskosten in seiner Bilanzierung zu aktivieren und demzufolge die AfA vorzunehmen. Die Leasingraten werden bei dem Leasinggeber als Betriebseinnahmen, bei dem Leasingnehmer als Betriebsausgaben betrachtet. Im Fall des Finanzierungs-Leasings wird der geleaste Gegenstand dem Leasingnehmer zugeschrieben. Demgemäß nimmt er auch die Abschreibung in seiner Bilanzierung vor. Weitere Unterschiede zwischen dem Operate- und Finanzierungs-Lasing betreffen den Umsatzsteueraspekt. Während das Finanzierungsleasing als Warenlieferung angesehen wird, mithin eine einmalige vorschüssige Steuerzahlung am Anfang der Grundmietzeit impliziert, wird das Operate-Leasing als Dienstleistung betrachtet. Die Umsatzsteuer wird in diesem Fall bei jeder einzelnen Leasingrate erhoben (vgl. Dz.U.04.54.535) ${ }^{40}$.

Für die Analyse des Leasings als eine Alternative für Finanzierung mittels Kredits wird eine Vergleichsrechung am Beispiel eines Schleppers aufgestellt. Der Vergleich basiert auf dem Barwert der Zahlungen beider Finanzierungsformen. Der obere Teil der Tabelle 7-2 zeigt einen Finanzierungsplan für ein Annuitätsdarlehen. Die hierzu getroffenen Annahmen werden im oberen Teil der Tabelle dargestellt. Der in diesem Beispiel in den Spalten III und IV ausgewiesene Kapitaldienst wird identisch wie in dem vorangegangenen Beispiel der

\footnotetext{
${ }^{40}$ Für eine ausführlichere Studie des Leasing in Polen siehe z.B. Martkowski, Kosmider 2004.
} 
Kreditfinanzierung ermittelt (siehe Tabelle 7-1). Die in der Spalte V ausgewiesene Steuerersparnis, wird unter Berücksichtigung der Fremdzinsen, der AfA und der USt berechnet. Zur Wahrung der Übersichtlichkeit werden die einzelnen Posten für die Ermittlung der Steuerersparnis getrennt in der Tabelle 7-3 dargestellt. In der Spalte VI der Tabelle 7-2 werden die Ausgaben nach Berücksichtigung der Steuerersparnis saldiert, um diskontiert und in einem weiteren Schritt in der Spalte VIII als Barwert dargestellt zu werden.

Tabelle 7-2: Vergleich der Finanzierung mithilfe von Kredit und Finanzierungsleasing (ZL)

\begin{tabular}{|c|c|c|c|c|c|c|c|}
\hline \multicolumn{8}{|c|}{ Investitionsfinanzierung mithilfe von Kredit } \\
\hline \multicolumn{8}{|c|}{$\begin{array}{l}\text { Annahmen: } \\
\text { - A = } 140000 \text { ZL inkl. USt } \\
\text { - Nennbetrag des Kredits = } 112000 \text { ZL }\end{array}$} \\
\hline Jahr & $\begin{array}{c}\text { Eigenkapita } \\
\text { und } \\
\text { Restschuld }\end{array}$ & Tilgung & Kreditzinsen & $\begin{array}{c}\text { Steuerersparnis } \\
\text { bei einem } \\
\text { Steuersatz von } \\
19 \% \\
\end{array}$ & $\begin{array}{l}\text { Saldo der } \\
\text { Ausgaben } \\
\text { III+IV-V }\end{array}$ & $\begin{array}{c}\text { Barwertfaktor } \\
\text { bei einem } \\
\text { Zinssatz von } \\
6 \% \\
\end{array}$ & $\begin{array}{c}\text { Barwert } \\
\text { der } \\
\text { Ausgaben } \\
\text { VI } \times \text { VII } \\
\end{array}$ \\
\hline $\mathrm{I}$ & II & III & IV & $\mathrm{V}$ & VI & VII & VIII \\
\hline & 28000 & - & - & - & 28000 & 1 & 28000 \\
\hline 1 & 112000 & 19868 & 6720 & 7527 & 19061 & 0,9434 & 17982 \\
\hline 2 & 92132 & 21060 & 5528 & 2504 & 24084 & 0,8900 & 21435 \\
\hline 3 & 71071 & 22324 & 4264 & 2264 & 24324 & 0,8396 & 20422 \\
\hline 4 & 48747 & 23664 & 2925 & 2009 & 24580 & 0,7921 & 19470 \\
\hline 5 & 25083 & 25083 & 1505 & 1739 & 24849 & 0,7473 & 18570 \\
\hline \multicolumn{7}{|c|}{ Barwert der Ausgaben für 60 Monate } & 125879 \\
\hline \multicolumn{8}{|c|}{ Investitionsfinanzierung mithilfe von Leasing } \\
\hline \multicolumn{8}{|c|}{$\begin{array}{l}\text { Annahmen: } \\
\text { - A = } 140000 \text { ZL inkl. USt } \\
\text { - Anzahlung = 28 000 ZL } \\
\text { - Erwerb des Schleppers am E }\end{array}$} \\
\hline Jahr & \begin{tabular}{c|c} 
Eigen- \\
kapital
\end{tabular} & $\begin{array}{c}\text { USt }+ \\
\text { Leasingraten }\end{array}$ & $\begin{array}{l}\text { Erwerb des } \\
\text { Objektes } \\
\text { inkl. USt }\end{array}$ & $\begin{array}{c}\text { Steuerersparnis } \\
\text { bei einem } \\
\text { Steuersatz von } \\
19 \% \\
\end{array}$ & $\begin{array}{l}\text { Saldo der } \\
\text { Ausgaben } \\
\text { (II) } \\
\text { III-V } \\
\end{array}$ & $\begin{array}{c}\text { Barwertfaktor } \\
\text { bei einem } \\
\text { Zinssatz von } \\
6 \% \\
\end{array}$ & $\begin{array}{c}\text { Barwert } \\
\text { der } \\
\text { Ausgaben } \\
\text { VI } \times \text { VII } \\
\end{array}$ \\
\hline $\mathrm{I}$ & II & III & IV & $\mathrm{V}$ & VI & VII & VIII \\
\hline & 28000 & - & & - & 28000 & 1 & 28000 \\
\hline 1 & & 26880 & & 5107 & 21773 & 0,9434 & 20540 \\
\hline 2 & & 26880 & & 5107 & 21773 & 0,8900 & 19378 \\
\hline 3 & & 26880 & & 5107 & 21773 & 0,8396 & 18280 \\
\hline 4 & & 26880 & & 5107 & 21773 & 0,7921 & 17246 \\
\hline 5 & & 26880 & 28000 & 5107 & 49773 & 0,7473 & 37195 \\
\hline- & & 1 für $60 \mathrm{~N}$ & & & & & 140258 \\
\hline
\end{tabular}

Quelle: eigene Berechnung in Anlehnung an Köhne 1981, S. 310

In dem unteren Teil der Tabelle 7-2 wird analog eine Leasingfinanzierung analysiert. Es wird hier unterstellt, dass der Leasingnehmer vor der Übernahme der Maschine eine Anzahlung von $20 \%$ des Anschaffungspreises einzureichen hat (II). Den Rest (112.000 ZL) finanziert er annahmegemäß mit Leasing. Die Höhe der Leasingraten inkl. USt wird in der Spalte III dargestellt. Es wird hier weiterhin angenommen, dass die Maschine nach dem Ablauf der Finanzierungsfrist von dem Leasingnehmer erworben wird (IV). Analog zu dem 
Kreditbeispiel wird in der Spalte V die Steuerersparnis dargestellt. Bei der Berechnung der Steuerersparnis sind die in der Spalte III berücksichtigten Raten wirksam. Die jährlichen Ausgaben nach Berücksichtigung der Steuerersparnisse werden abgezinst und in der Spalte VIII als Barwert ausgedrückt.

Aus dem dargestellten Vergleich geht hervor, dass die Finanzierung durch Inanspruchnahme des Kredites wesentlich günstiger ist als Leasing. Dieser Vergleich ist allerdings keine hinreichende Grundlage für allgemeingültige Aussagen und dient vielmehr als ein methodisches Beispiel.

Tabelle 7-3: Grunddaten zur Ermittlung der Steuerersparnis beim Kauf (ZL)

\begin{tabular}{|c|c|c|c|c|}
\hline \multicolumn{5}{|c|}{$\begin{array}{l}\text { Annahmen: } \\
\text { - lineare AfA = } 15 \text { Jahre } \\
\text { - USt }=22 \%\end{array}$} \\
\hline Jahr & USt und AfA & Kreditzinsen & $\begin{array}{c}\text { Summe der Spalten } \\
\text { II und III }\end{array}$ & $\begin{array}{l}\text { Steuerersparnis bei einem } \\
\text { Steuersatz von } 19 \%\end{array}$ \\
\hline $\mathrm{I}$ & II & III & IV & $\mathrm{V}$ \\
\hline 1 & $25246+7650$ & 6720 & 39616 & 7527 \\
\hline 2 & 7650 & 5528 & 13178 & 2504 \\
\hline 3 & 7650 & 4264 & 11914 & 2264 \\
\hline 4 & 7650 & 2925 & 10575 & 2009 \\
\hline 5 & 7650 & 1505 & 9155 & 1739 \\
\hline
\end{tabular}

Quelle: eigene Berechnung in Anlehnung an Köhne 1981, S. 310

\section{Weiterführende Überlegungen}

Bei der Analyse der Vorteilhaftigkeit verschiedener Finanzierungsformen sind unterschiedliche Ausgangsbedingungen zum Zeitpunkt der Investition in den landwirtschaftlichen Betrieben zu berücksichtigen. Dabei handelt es sich im Wesentlichen um das Volumen des disponiblen Eigenkapitals, seine Rendite bei Verwendung in bestimmten Investitionen und um den Umfang sowie die Höhe der Verzinsung des zugänglichen Fremdkapitals. Im Fall, in dem das vorhandene Eigenkapital nicht effizienter als bei der geplanten Investition eingesetzt werden kann oder bei seiner Nichtanwendung höchstens eine Verzinsung einer konventionellen Geldanlage erreichbar ist, empfiehlt es sich, einen möglichst hohen Selbstfinanzierungsgrad anzustreben. Der Grund hierfür ist leicht verständlich und besteht darin, dass der Zinsanspruch bei der Aufnahme von Fremdkapital im Normalfall über der Verzinsung des Eigenkapitals liegt.

In der Mehrheit der landwirtschaftlichen Betriebe in Polen ist es selten der Fall, dass die Investitionen vollständig oder auch zum großen Teil aus den Eigenmitteln finanziert werden können. Die Mehrzahl der Betriebe ist auf die Außenfinanzierung angewiesen. Aufgrund des 
Bodeneigentums ist in den landwirtschaftlichen Betrieben der Beleihungsspielraum im Regelfall ausreichend, um die geplanten Investitionen vollständig mithilfe von Fremdmitteln zu finanzieren. Dabei handelt es sich in erster Linie um die Finanzierung mittels Kredits. Bei der Auswahl eines konkreten Kredits aus der Anzahl aller zugänglichen Kredite sind Belastungsvergleiche erforderlich, in denen alle mit der Finanzierung verbundenen Zahlungen berücksichtigt werden. Es ist dabei zu beachten, dass im Normalfall eine Ausgabe eine umso geringere Belastung darstellt, je weiter sie hinausgeschoben wird. Der an dieser Stelle eintretende Zinsvorteil gegenüber einer früher fälligen Ausgabe ist durch die Abzinsung der jeweiligen Beträge auf den Zeitpunkt der Investitionsvornahme zu berücksichtigen. Bei Einnahmen gilt, im Hinblick auf den Eintrittstermin eine umgekehrte Beziehung, d.h. je früher sie anfallen, desto größer ist ihre Bedeutung. Daher sind auch die Steuerersparnisse auf den Zeitpunkt der Investitionsvornahme zu diskontieren. Die Summe der Salden jedes einzelnen Jahres, die in den vorangegangenen Beispielsrechnungen auf den Zeitpunkt der Anschaffung der Maschine abgezinst werden, stellt den Maßstab für den Vergleich verschiedener Finanzierungsvarianten dar. Die Finanzierungsform mit dem geringsten Barwert der Ausgaben ist nach Berücksichtigung der Steuerersparnisse aus Kostensicht am günstigsten.

Wie ohne weiteres einleuchtet, sind die zinsverbiligten Kredite im Vergleich mit traditionellen Krediten billiger. Der Kostenvorsprung bei diesen Krediten kann zusätzlich durch die Berücksichtigung der tilgungsfreien Frist vergrößert werden.

Als eine Alternative für eine vollständige Fremdfinanzierung kann Leasing angesehen werden. Es ist dabei schon eingangs zu bemerken, dass Leasing bezüglich der finanziellen Belastung im Vergleich mit einer vollständigen Kreditfinanzierung im Normalfall deutliche Nachteile aufweist. Dies ist verständlich und gründet sich darin, dass die Leasingunternehmen ihre Finanzierungsraten auf der Grundlage der Kreditkonditionen mit einem bestimmten Zuschlag für Risikoabdeckung und eigenen Gewinn festlegen. Wenngleich die Leasingunternehmen bei den Maschinenherstellern besonders günstiger Rabatte erhalten können und somit die Möglichkeit haben, die Maschinen zu einem billigeren Preis anzubieten, ist die Leasingfinanzierung i.d.R. teurer als Kredit. Bei Beurteilung des Leasings sollen jedoch noch andere Aspekte mit berücksichtigt werden. Dabei handelt es sich zunächst um die Zusammensetzung der Finanzierungsmittel. In dem dargestellten Beispiel der Leasingfinanzierung wurde eine Anzahlung von $20 \%$ des Anschaffungspreises, fällig am Anfang der Grundmietzeit unterstellt. Es wurde dadurch zwar der Effekt erzielt, die künftigen 
Mietraten zu vermindern, die anfängliche Anzahlung kann jedoch aus der Sicht der finanziellen Belastung dem Einsatz von Eigenkapital gleich gestellt werden. In der Praxis besteht die Möglichkeit, eine Investition zu hundert Prozent durch Leasing ohne Selbstanteil zu finanzieren. Dies kann vorteilhaft für jene Betriebe sein, die nicht ausreichend Eigenkapital besitzen und gleichzeitig keine Möglichkeit haben, die Investitionen vollständig durch einen Kredit zu finanzieren.

Von den Leasingfirmen wird oft der Aspekt der Besicherung der Investition als Leasingvorteil hervorgehoben. Während bei Bankdarlehen im Regelfall die Grundschuld den Sicherungsrahmen bildet, reicht als Sicherung bei Leasing der geleaste Gegenstand aus. Dies kann von Bedeutung in den Betrieben sein, in denen der Beleihungsspielraum eingeschränkt ist. Es handelt sich dabei z.B. um Pachtbetriebe, die keine ausreichende Sicherung der Investition herstellen können. In der Mehrheit der Betriebe, die aufgrund des Bodeneigentums über einen großen Spielraum verfügen, hat das angesprochene Argument jedoch kaum Aussagekraft.

Des Weiteren wird als besonderer Vorteil des Leasings die Steuerersparnis herausgestellt. Ausgehend von einer ordnungsmäßigen Buchführung, der in der polnischen Landwirtschaft zzt. nur eine marginale Bedeutung zukommt, können hier die Einkommensteuerersparnisse interessant werden. Während bei Finanzierung mittels eines Kredits nur die AfA und Kreditzinsen steuerlich wirksam sind, können bei Leasing, wie in dem kalkulatorischen Beispiel dargestellt wurde, die gesamten Leasingraten abgesetzt werden. Vor diesem Hintergrund kann die Leasingfinanzierung für die Unternehmen von Bedeutung sein, die besonders kapitalaufwendige Investitionen vornehmen und dank den Leasingraten potenzielle Vorteile bei der Einskommensteuer besser nutzen können. Im Normalfall ist der Vorsprung in den Steuerersparnissen des Leasings vs. Finanzierung mittels Kredits nicht hinreichend, um dem Leasing den Vorzug zu gewähren.

Schließlich kann ein Vorteil des Leasings darin bestehen, dass hier der Beleihungsspielraum nicht eingeengt und u.U. das Eigenkapital geschont wird. Dies kann besondere Bedeutung in stark expandierenden Betrieben haben, in denen sowohl das Eigenkapital als auch die zugänglichen Kredite ausgeschöpft sind und weiterhin ein Investitionsbedarf besteht. Dabei ist allerdings $\mathrm{zu}$ bemerken, dass das Leasings von Vorteil nur in Bezug auf den Beleihungsspielraum aber nicht auf die Liquidität ist. 


\subsection{Gebrauchs- und zeitabhängiges Nutzungsdaueroptimum}

Bei der Modernisierung der Mechanisierung entsteht nicht nur die Frage, welche Maschinen, einzelbetrieblich oder kooperativ angeschafft oder wie sie finanziert werden sollten, sondern auch wann sie zu ersetzen sind. Auf dieses Problem wird im Folgenden eingegangen.

Für die Bestimmung der optimalen Ersatzstrategie dauerhafter Produktionsmittel werden in der Praxis zwei Kriterien herangezogen. Es wird zunächst der Einfluss des technischen Verschleißes auf die Leistungsfähigkeit der Maschinen bestimmt. Entscheidend dabei ist der allgemeine technische Zustand, der die Abnutzung bestimmter Maschinenteile widerspiegelt. Da die ausfallenden Einzelteile einer Maschine eigentlich stets ersetzt oder repariert werden können, wäre die unter ausschließlicher Berücksichtigung dieses Kriteriums ermittelte Nutzungsdauer beliebig zu verlängern (vgl. SCHAEFER 1969, S. 18 ff.). Aus diesem Grund werden in die Überlegungen über die optimale Nutzungsdauer oder den optimalen Ersatzzeitpunkt einer Maschine zusätzlich wirtschaftliche Aspekte einbezogen. Es handelt sich hier im Wesentlichen um die Arbeitsqualität, das mit dem Alter steigende Ausfallrisiko, die zunehmenden Wartungs- und Reparaturausgaben, die Ernteverluste und die zeitliche Entwicklung der Wiederveräußerungswerte der gebrauchten Maschinen. Eine wesentliche Bedeutung hat bei der Entscheidungsfindung auch der technische Fortschritt und die damit verbundene Möglichkeit, moderne Verfahren anzuwenden.

Die Methodik zur Ermittlung der optimalen Ersatzstrategie bei Maschinen beruht im Wesentlichen auf zwei Standardansätzen. Es handelt sich dabei zum einen um die ex ante Kalkulation, die auf der Untersuchung der Durchschnittskosten einer, zumeist neu gekauften Maschine basiert und zum anderen um die Methode, bei der die Grenzkosten einer seit bestimmter Zeit im Betrieb eingesetzten Maschine analysiert werden.

Gemäß der Methode der Durchschnittskosten, wird die optimale Nutzungsdauer einer Maschine in dem Stadium erreicht, in dem die Durchschnittskosten bei gegebener Leistung ihr Minimum erreichen. Dies ist der Fall, wenn die Grenzkosten, also die Kosten des letzten Abschnitts einer bestimmten Periode bestehend aus Reparaturen, Betriebsstoffen und zahlenmäßig ausgedrücktem Wertverlust, die Durchschnittskosten schneiden (vgl. BRANDES, ODENING 1992, S. 56 ff.). In dieser Methode wird vereinfacht davon ausgegangen, dass eine Maschine nach Ende ihrer Nutzungsdauer, durch eine aus der Kostensicht identische Investition ersetzt wird. Dies führt zur Annahme eines sich in gleicher Weise wiederholenden Zahlungsstroms und lässt den technischen Fortschritt sowie die daraus 
resultierende Kostenwirkung unberücksichtigt. Die Zuverlässigkeit dieser Methode hängt von der Abbildung des Reparaturkostenverlaufs und der Restwertfunktion ab. Ein Beitrag zur Lösung des Problems der Abbildung des Reparaturkostenverlaufs wurde 1977 von REISCH und ZEDDIES dargestellt (1977, S. 78). Es wurde hierzu, basierend auf den Daten von SCHAEFER (1963, S. 41 ff.) ein beispielhaftes Modell entwickelt, wo reparaturfreie Jahre mit unterschiedlich hohen und sogar den Restwert erhöhenden Reparaturen kombiniert werden. In diesem Modell wird aus Vereinfachungsgründen, praxisfern eine über die gesamte Nutzungsdauer konstante Leistung der Maschinen unterstellt. Sowohl aus diesem Grund als auch wegen des Zeitbezuges der angewandten Daten kann dieses Modell lediglich als ein methodisches Beispiel betrachtet werden. Bei dieser Methode ist weiterhin zu bemerken, dass sie wegen der angesprochnen Schwierigkeiten in der Abbildung der Wiederveräußerungswerte, des Reparaturkostenverlaufs, in der Quantifizierung der von Fall zu Fall unterschiedlichen altersbedingten Betriebsrisiken und der Prognose des technischen Fortschritts nur in einem eingeschränkten Maße als eine Orientierungshilfe bei langfristiger Planung der Ersatzstrategie dienen kann. Der praktische Wert dieser Methode besteht v.a. in der Möglichkeit der Untersuchung der simultanen Auswirkungen auf die optimale Nutzungsdauer solcher Faktoren wie: der Zinssatz, die Restwertfunktion oder der Reparaturkostenverlauf in Abhängigkeit von der Auslastung der Maschinen (vgl. KÖHNE 1980, S. 257 ff.).

Mit der Methode der Grenzkosten wird untersucht, ob eine im landwirtschaftlichen Betrieb seit bestimmter Zeit vorhandene Maschine weiter genutzt oder durch eine neue Investition oder evtl. Zukauf von Dienstleistung ersetzt werden soll. Es werden dabei die Grenzkosten der vorhandenen Maschine den Durchschnittskosten einer Alternative gegenübergestellt. Der wirtschaftlich optimale Ersatzzeitpunkt wird in dieser Phase erreicht, in der die Grenzkosten der analysierten Maschinen die Durchschnittskosten der vorhandenen Alternative übersteigen.

Während die erste der geschilderten Methoden eine gewisse Bedeutung bei den Prognosen der optimalen Nutzungsdauer haben kann und nur in einem beschränkten Maße der Praxis dient, ist die Grenzkostenmethode ein bewährte Orientierungshilfe, auf dem Niveau der landwirtschaftlichen Betriebe der Frage nachzugehen, wie lange eine Maschine bei neu auftretenden Anlässen genutzt werden soll. 


\subsection{Beispielkalkulationen für die Ermittlung der optimalen Nutzungsdauer der Maschinen}

Es wird im Folgenden die Methodik der Ermittlung einer optimalen Ersatzstrategie am konkreten Beispiel des Schleppers aus den früheren Kalkulationen kurz geschildert. Diese Überlegungen werden in einem weiteren Schritt um den Aspekt der Steuern und Inflation erweitert. Der Einfluss von Steuern und Inflation auf die optimale Nutzungsdauer von Maschinen wurde in der deutschen Fachliteratur intensiv erörtert. Es wurden dabei Modellkalkulationen entworfen, anhand von denen die Wirkung beider Faktoren auf die optimale Ersatzstrategie quantifiziert wird (vgl. z.B. KöHNE 1980, S. 257 ff., MILCH 1982, S. 309 ff.). Auf diese Methodik wird im Weiteren nicht eingegangen. Das Ziel der folgenden Ausführung ist es, lediglich die Ansatzpunkte für die Überlegungen zur optimalen Nutzungsdauer im Inflations- und Steuerfall deskriptiv zu schildern.

\section{Durchschnittskostenmethode}

Für die Darstellung der Methode ist auf die Tabelle 7-4 zu verweisen. Die hier unterstellten Wiederveräußerungswerte werden in Anlehnung an die Schwackeliste gemäß einer geometrischen Folge aus mittlerer Entwertung der von Preis und Motorleistung ähnlichen Schlepper ermittelt (vgl. REIMERS 1996, S. 116 ff.). Die jährlichen Kosten für Betriebsstoffe, Versicherung und Unterbringung werden als von der Nutzungsdauer unabhängige Größen nicht in die Kalkulation einbezogen. Die unterstellten Reparaturkosten wurden aus den KTBL Betriebsdaten übernommen und wie vorher erläutert, modifiziert. Es wird an dieser Stelle versucht, der Progression dieser Kosten mit steigendem Abnutzungsgrad Rechnung zu tragen. Die jeweiligen Wiederveräußerungswerte und die anfallenden Reparaturen werden für jedes einzelne Jahr diskontiert und in den Spalten V und VII dargestellt. In der Spalte VIII werden die diskontierten Reparaturen bis zum Ende der jeweiligen Nutzungsdauer des Schleppers zusammengerechnet. Die Zahlen der Spalte IX geben den Kapitalwert der Ein- und Auszahlungen für jeden einzelnen Abstand der Nutzungsdauer wieder. So errechnet sich beispielhaft die Zahl der ersten Zeile als 140.000-105.661 + 472. Die in der letzten Spalte ausgewiesen Durchschnittskosten (DK) stellen den Wert aus der Multiplizierung der Kapitalwerte für jedes einzelne Jahr der Nutzungsdauer mit dem entsprechenden Wiedergewinnungsfaktor (WF) dar. In diesem Beispiel fällt der optimale Ersatzzeitpunkt des Schleppers auf das 12. Jahr. 
Tabelle 7-4: Beispiel zur Ermittlung der optimalen Nutzungsdauer eines Schleppers mithilfe der Durchschnittskostenmethode

\begin{tabular}{|c|c|c|c|c|c|c|c|c|c|}
\hline \multicolumn{10}{|c|}{$\begin{array}{l}\text { Annahmen: } \\
\mathrm{A}=140000, \mathrm{i}=6 \%, \mathrm{~N}=15 \text { Jahre }\end{array}$} \\
\hline $\mathrm{I}$ & II & III & IV & $\mathrm{V}$ & VI & VII & VIII & IX & $\mathrm{X}$ \\
\hline Jahr & DF & WF & $\begin{array}{c}\text { Wiederver- } \\
\text { äußerungswert }\end{array}$ & $\mathrm{IV} \times \mathrm{DF}$ & $\begin{array}{l}\text { Repara- } \\
\text { turkosten } \\
\text { je Jahr }\end{array}$ & $\mathrm{VI} \times \mathrm{DF}$ & $\begin{array}{c}\text { Kumu- } \\
\text { liert }\end{array}$ & $\begin{array}{c}\text { Kapitalwert } \\
\text { der Ein- und } \\
\text { Auszahlungen }\end{array}$ & $\begin{array}{c}\mathrm{DK} \\
\mathrm{III} \times \mathrm{IX}\end{array}$ \\
\hline 1 & 0,9434 & 1,0600 & 112000 & 105661 & 500 & 472 & 472 & 34811 & 36900 \\
\hline 2 & 0,8900 & 0,5454 & 96000 & 85440 & 1000 & 890 & 1362 & 55922 & 30500 \\
\hline 3 & 0,8396 & 0,3741 & 82000 & 68847 & 1500 & 1259 & 2621 & 73774 & 27599 \\
\hline 4 & 0,7921 & 0,2886 & 71200 & 56398 & 2000 & 1584 & 4206 & 87808 & 25341 \\
\hline 5 & 0,7473 & 0,2374 & 62000 & 46333 & 2500 & 1868 & 6074 & 99741 & 23679 \\
\hline 6 & 0,7050 & 0,2034 & 53200 & 37506 & 3500 & 2468 & 8541 & 111035 & 22585 \\
\hline 7 & 0,6651 & 0,1791 & 44800 & 29796 & 4500 & 2993 & 11534 & 121738 & 21803 \\
\hline 8 & 0,6274 & 0,1610 & 39200 & 24594 & 5500 & 3451 & 14985 & 130391 & 20993 \\
\hline 9 & 0,5919 & 0,1470 & 34000 & 20125 & 6500 & 3847 & 18832 & 138708 & 20390 \\
\hline 10 & 0,5584 & 0,1359 & 29600 & 16529 & 7500 & 4188 & 23020 & 146492 & 19908 \\
\hline 11 & 0,5268 & 0,1268 & 25600 & 13486 & 10500 & 5531 & 28552 & 155066 & 19662 \\
\hline 12 & 0,4970 & 0,1193 & 22000 & 10934 & 14500 & 7207 & 35758 & 164824 & 19664 \\
\hline 13 & 0,4688 & 0,1130 & 18800 & 8813 & 18500 & 8673 & 44431 & 175618 & 19845 \\
\hline 14 & 0,4423 & 0,1076 & 14500 & 6413 & 23500 & 10394 & 54825 & 188412 & 20273 \\
\hline 15 & 0,4173 & 0,1030 & 12000 & 5008 & 28000 & 11684 & 66510 & 201502 & 20755 \\
\hline
\end{tabular}

Quelle: eigene Berechnung in Anlehnung an Köhne 1980, S. 257 ff.

\section{Grenzkostenmethode}

Für das Beispiel der Grenzkostenmethode wird derselbe Datensatz angewendet. Es wird als Kalkulationszeitpunkt das achte Nutzungsjahr des Schleppers gewählt. In der Tabelle 6-18 sind zunächst die Kosten des weiteren Einsatzes des Schleppers vom Ende des achten Einsatzjahres nach der Anschaffung in den drei folgenden Jahren analysiert. In dem unteren Teil der Tabelle werden getrennt die Kosten für das 12. Nutzungsjahr aufgezeigt. Als Alternative wird ein identisches Schlepperfabrikat unterstellt. Die durchgeführte Kalkulation veranschaulicht, dass eine Nutzung der Maschine in drei weiteren Jahren vom Ende des achten Jahres ausgehend wirtschaftlich angebracht ist. Die Untersuchung der Kosten, die im zwölften Jahr anfallen, zeigt demgegenüber, dass eine weitere Nutzung des Schleppers im Verhältnis zur vorhandenen Alternative höhere Kosten verursacht. Dementsprechend ist gemäß der Kalkulation die Inanspruchnahme der Alternative vorzuziehen. 
Tabelle 7-5: Beispiel zur Ermittlung der optimalen Nutzungsdauer eines Schleppers mithilfe der Grenzkostenmethode für drei Nutzungsjahre und ein weiteres Nutzungsjahr

\section{Durchschnittliche Grenzkosten für 3 Jahre}

Kapitalwert der Ein- und Auszahlungen

$39200-\frac{25600}{1,06^{3}}+\frac{6500}{1,06}+\frac{7500}{1,06^{2}}+\frac{10500}{1,06^{3}}=39328,80$

Wertminderung abgezinste Reparaturen

Grenzkosten pro Jahr $39328,80 \times \mathrm{WF}_{6 ; 3}=14712,90$

Grenzkosten für zwölftes Jahr

AfA $(22000-18800) \quad 3200$

$\mathrm{Z}_{\mathrm{i}} \quad(22000 \times 0,06) \quad 1320$

RK 18500

¿Grenzkosten $\quad 23020$

Minimale Kosten der Alternative 19662 ZL/Jahr

Quelle: eigene Berechnung in Anlehnung an Köhne 1980, S. 257 ff.

\section{Einfluss von Steuern und Inflation}

Eine wesentliche Bedeutung bei der Bestimmung der optimalen Nutzungsdauer und somit der Umschlaghäufigkeit der Maschinen können Einkommensteuer und Inflation haben. Von einer ordnungsgemäßen Buchführung ausgehend, kann die Berücksichtigung von Steuern in die Richtung der Verkürzung der Nutzungsdauer wirken. Dies gründet sich zunächst darin, dass die in der Buchführung ausgewiesen AfA und Reparaturen als Betriebsausgabe gelten und somit zu einer Steuerersparnis führen. Bei Fremdfinanzierung können auch die Kapitalzinsen steuerlich aktiviert werden. An dieser Stelle wichtig ist auch die AfA- Methodik. In der deutschen Landwirtschaft handelt es sich dabei um die degressive Abschreibung und die Sonderabschreibung gemäß §7g EStG (siehe hierzu auch S. 29 ff.). Diese Instrumente dringen tendenziell auf die Verkürzung der Nutzungsdauer der Maschinen.

Unter Berücksichtigung von Inflation tritt gegenüber einer einfachen Kalkulation ein Effekt ein, der ebenso zur Verkürzung der optimalen Nutzungsdauer führt. Von Bedeutung ist dabei die Relation der Reparaturkosten, der Abschreibung und der Wiederveräußerungswerte. Im Inflationsfall ist im Zeitablauf eine relative Steigerung der Reparaturkosten zu verzeichnen. Diesem steht eine Abschwächung des Gewichtes der Abschreibung gegenüber, weil die Wiederveräußerungswerte steigen. Eine Änderung der Kostenrelation zugunsten der Reparaturkosten kann einen Ausschlag für einen früheren Maschinenersatz geben. An dieser Stelle ist allerdings auch die Entwicklung des Zinsniveaus zu bedenken. Mit zunehmender Inflationsrate geht i.d.R. ein wachsendes Zinsniveau einher. Der Anstieg der Kreditzinsen führt zur Erhöhung der Kapitalkosten und tendenziell einer Verlängerung der optimalen 
Nutzungsdauer. Der Effekt der in die Richtung der Verkürzung der Nutzungsdauer wirkenden Inflation kann dadurch gemindert, möglicherweise ganz nivelliert werden (vgl. KöHNE 1980, S. 262).

\subsection{Zusammenfassung}

Die Mehrheit der landwirtschaftlichen Betriebe in Polen ist aufgrund niedriger Ertragslage nicht imstande, die Entwicklungsinvestitionen aus eigener Kraft zu finanzieren. Vor diesem Hintergrund wird auch die staatliche investive Förderung aufgebaut. Sie hat zum Ziel, über die Steigerung der Kapitalausstattung die Wachstumschancen der landwirtschaftlichen Betriebe zu erhöhen. Ein integrierter Bestandteil der Förderungspolitik in der polnischen Landwirtschaft ist die finanzielle Begünstigung von Investitionen in die technische Ausstattung. Die Förderung wird in Form einer Zinsverbilligung für Kapitalmarktdarlehen und eines verlorenen Zuschusses gewährt. Als Hauptentscheidungsträger bei der Vergabe der Fördermittel agiert die ARiMR. In den Entscheidungsprozess werden auch die örtlichen Beratungsstellen und Banken einbezogen. Die Vergabe der Fördermittel erfolgt nach bestimmten Kriterien unter Berücksichtigung der regionalen Differenzen der Landwirtschaftstruktur. Es liegen bis dato keine empirisch bestätigten Ergebnisse einer Kausalanalyse der Wirkung der getroffenen Förderungsmaßnahmen und den ihnen zugrunde gelegten Zielen der Landwirtschaftsentwicklung vor.

Die Auswahl einer am besten geeigneten Form der Investitionsfinanzierung setzt einen Vergleich der zugänglichen Finanzierungsformen voraus. Dies soll vor dem Hintergrund der betriebsindividuellen Gegebenheit erfolgen. Dabei $\mathrm{zu}$ bedenken sind u.a. die Eigenkapitalrendite, die Betriebsstabilität oder die Zeitpunkte der Realisierung der Verkaufserlöse sowie die damit verbundenen temporalen Unterschiede in der Liquiditätsfähigkeit. Beim Vergleich verschiedener Kredite bietet sich zunächst das Aufstellen eines Zins- und Tilgungsplanes an. Wichtig ist dabei eine sorgfältige Beachtung der Finanzierungskonditionen und Ermittlung des effektiven Zinsfusses. Es ist in der Regel die Finanzierungsform $\mathrm{zu}$ bevorzugen, bei der neben der Deckung des erforderlichen Kapitalbedarfs die niedrigsten Zinsen zu zahlen sind. Es ist grundsätzlich die Inanspruchnahme der Mittel aus dem Programm der investiven Förderung zu empfehlen. Dabei darf aber der Geschichtspunkt der Wirtschaftlichkeit nicht hintangestellt werden. Der Zugang zu Fördermitteln darf nicht zu Investitionen verleiten, die nicht in die realen Entwicklungspläne des Betriebes passen oder zur Entstehung von Überkapazitäten führen. 
Bei der Auswahl einer Finanzierungsform soll auch das Leasing mit berücksichtigt werden. Es ist dabei allerdings schon vorweg anzumerken, dass dieses Finanzierungsinstrument i.d.R. teurer ist als Bankdarlehen. Das Argument der steuerlichen Wirkung des Leasings trifft für die Mehrheit der landwirtschaftlichen Betriebe generell nicht zu. Das Leasing kann jedoch durchaus vorteilhaft für jene Betriebe sein, die aufgrund anstehender kapitalaufwendiger Entwicklungsinvestitionen den Kreditspielraum ausschöpfen und die weiter erforderlichen Investitionen aus anderen Mitteln finanzieren müssen.

Für die Rentabilität des Maschineneinsatzes und die Sicherung der Entwicklungsfähigkeit der landwirtschaftlichen Betriebe ist eine optimale Ersatzstrategie der Maschinen von wesentlicher Bedeutung. Eine Orientierungshilfe bei der Ermittlung der optimalen Nutzungsdauer der Maschinen bieten die dargestellten Kalkulationen. Allein aufgrund der kalkulatorischen Ergebnisse können allerdings keine endgültigen Aussagen getroffen werden. Bei der Fragestellung nach dem optimalen Ersatzzeitpunkt sind der technische Fortschritt, die Restewertfunktion, das mit dem zunehmenden Alter der Maschinen ansteigendes Ausfallrisiko und die weitere Betriebsentwicklung $\mathrm{zu}$ bedenken. So kann die optimale Ersatzstrategie der Maschinen nur betriebsspezifisch unter einer sorgfältigen Untersuchung aller Einflussfaktoren gewählt werden. 


\section{Schlussbetrachtungen}

\subsection{Kritische Stellungnahme}

Das Ziel der vorliegenden Arbeit ist es, zu untersuchen, inwieweit die deutschen Erfahrungen mit der Entwicklung verschiedener Formen der überbetrieblichen Zusammenarbeit im Maschineneinsatz als potenzielle Orientierungshilfe für die weitere Mechanisierung der landwirtschaftlichen Betriebe in Polen dienen können. In Bezug auf die hierzu in den Kapiteln 5 und 6 vorgenommenen Ausführungen ist im Folgenden kritisch Stellung zu dem konzeptionellen Hintergrund der Vorgehensweise zu nehmen. Die Erörterungen beziehen sich zunächst auf die allgemeinen Aspekte der Landwirtschaftstruktur der Untersuchungsregionen, weiterhin speziell auf die ausgewählten Betriebe als Untersuchungsgegenstand.

Nicht zu unterschätzen bei den Überlegungen zur künftigen Mechanisierung beider Untersuchungsregionen ist die Bedeutung des möglichen Strukturwandels. Mittels komplexer Simulationsmodelle konnte bereits bewiesen werden, dass unterschiedliche Landwirtschaftsstrukturen, einschließlich dualer Strukturen, bei verschiedenen Rahmenbedingungen, über längere Zeit Bestand haben können (vgl. LinNEMANN 2001, S. 133 ff., m.w.N.). Die Untersuchung des möglichen Strukturwandels, seines Charakters und des Tempos ist insofern wichtig, dass sie wesentliche Ansatzpunkte für die Planung der regional differenzierten Förderungsmaßnahmen für die Entscheidungsträger aus der Politik und den Institutionen des landwirtschaftlichen Umfelds bilden kann. Für beide Untersuchungsregionen kann ein unterschiedlicher Verlauf der Strukturänderungen angenommen werden. Während in der nördlichen Region günstige Bedingungen für eine weitere Entwicklung von großen spezialisierten Betrieben vorliegen, ist die Entwicklung der Betriebe in der Region Lublin stark durch die Flächenzersplitterung determiniert. $\mathrm{Zu}$ Bedenken ist im Fall des Gebiets Lublin auch die Bedeutung der direkten Nähe des großen Ballungszentrums der gleichnamiger Stadt Lublin. Bei der Formulierung der Schlussfolgerungen wird versucht, den Aspekten der regionalen Gegebenheiten hinreichend Rechnung zu tragen. Es werden dabei die Aussagen der befragten Landwirte und Berater bezüglich weiterer Betriebsentwicklungen und die Altersstruktur der Betriebsleiter berücksichtigt. Die dargestellten Ergebnisse basieren jedoch nicht auf einer komplexen Untersuchung und haben den Charakter allgemeiner Tendenzbeschreibungen.

Als Gegenstand der vorliegenden Untersuchung sind Betriebe ausgewählt worden, die von den Beratern vor Ort als repräsentativ für die Regionen eingestuft wurden. Die ausgewählten 
Betriebe werden als Repräsentanten bestimmter Betriebsgrößengruppen in Bezug auf die Produktionsfaktoren (AK- und technische Ausstattung) und die Produktionsverfahren betrachtet.

In der nördlichen Region wurden Betriebe mit einer Flächenausstattung von 27 ha LF bis zu 335 ha LF befragt. Die Gründe für die Bildung der Stichprobe wurden bereits in Kapitel 5.4 erläutert. Es wurde dadurch ein homogenes, insgesamt ungünstiges Bild der technischen Ausstattung der landwirtschaftlichen Betriebe der Untersuchungsregion dargestellt. Darauf basieren auch die Vorschläge zu praktikablen Kooperationsformen. Bei Erweiterung der Stichprobe um größere Betriebe mit deutlich besserer technischer Ausstattung könnte das Gesamtbild verändert werden. Unter diesen Bedingungen wären die Aspekte der Aktivierung des Austausches der Dienstleistung zwischen Betrieben bestimmter Größengruppen intensiver $\mathrm{zu}$ analysieren.

In der Region Lublin wurden in die Untersuchung auch die Betriebe mit einer Flächenausstattung von weniger als 10 ha LF einbezogen. Ein Teil dieser Betriebe betreibt so gut wie keine Marktproduktion und kann somit zu den Subsistenzwirtschaften gezählt werden. Die Besitzer dieser Betriebe orientieren sich bei weiteren Entwicklungsentscheidungen nur in begrenztem Maße an Rahmenbedingungen der Landwirtschaft. Es wirft ein wesentliches Problem auf, diese Betriebe bei Überlegungen über den weiteren Strukturwandel in dieser Region eindeutig zu positionieren. Ausgehend von der Persistenz der gegenwärtigen Strukturen, können sie durchaus als wichtige Akteure des überbetrieblichen Maschineneinsatzes betrachtet werden. Gesetzt den Fall, dass diese Betriebe die landwirtschaftliche Produktion bald einstellen und ihre Flächen entweder verkauft oder verpachtet werden, können sie in die Überlegungen als Quelle freiwerdender Flächen und nicht als selbständige Objekte einbezogen werden. Für eine exakte Analyse der Eignung bestimmter Kooperationsformen im Bereich der Mechanisierung wäre es weiterhin nötig, den Betrachtungsraum $\mathrm{zu}$ erweitern und die angesprochenen Aspekte des künftigen Strukturwandels detaillierter zu untersuchen.

Die dargestellten Kosten der Mechanisierung bei der in den Betrieben beider Stichproben zzt. vorhandenen Ausstattung basieren im Wesentlichen auf den Aussagen der befragten Landwirte. Neben dem Reparaturkostenverlauf wichtig sind hier auch die Wiederveräußerungswerte. In Bezug auf die letzteren hat sich gezeigt, dass die Ergebnisse der Betriebsbefragung wesentlich von den Informationen der Berater und aus den 
Fachzeitschriften abweichen. Um dieser Tatsache Rechnung zu tragen, wurden die von den Landwirten angegebenen Werte vor dem Einbeziehen in die Kalkulationen der Realität angepasst. Im Fall der Reparaturkosten gibt es keine empirisch abgesicherten Daten. Daher konnten diese Kosten bei der zahlenmäßigen Analyse der bisherigen Mechanisierungsstruktur ausschließlich anhand der Einschätzungen der Landwirte erfasst werden.

Die exemplarischen Kalkulationen der zweckmäßigen Maschinen fußen auf den Standarddaten vom KTBL, die einigen Modifizierungen unterzogen wurden. Die Gründe für die Datenauswahl und weitere Vorgehensweise bei den Kostenkalkulationen werden in Kapitel 6.4.3 geschildert. Es wird dabei versucht einen realistischen Datensatz zu entwickeln. Die darauf aufgebauten Kalkulationen stellen allerdings lediglich eine Leitlinie dar, die bei veränderten Daten zu unterschiedlichem Ergebnis führen kann.

Die Auswahl zweckmäßiger Maschinen unter Berücksichtigung der für jede Region geeigneten Kooperationsformen erfolgte vor dem Hintergrund der aktuellen Rahmenbedingungen der befragten Betriebe. Eine besondere Bedeutung wird dabei der Darstellung der praktikablen Verbesserungen beigemessen. Dies kommt auch zum Ausdruck bei der Analyse der Kosten der Gebrauchtmaschinen. Dabei handelt es sich um Maschinen, die bei anstehenden Ersatzinvestitionen angeschafft und unter den vorliegenden Rahmenbedingungen effizient eingesetzt werden können. Es wird hier nicht der Versuch unternommen, eine Prognose der Entwicklung der Mechanisierung für längere Zeit unter Berücksichtigung der möglichen Effekte des Strukturwandels darzustellen. Dies betrifft nicht zuletzt auch die professionellen Lohnunternehmen mit moderner schlagkräftiger Technik, deren Entwicklungschancen nicht anhand von beispielhaften Kalkulationen, sondern lediglich deskriptiv in die Betrachtungen einbezogen wurden.

Die Aspekte der Investitionsfinanzierung werden in Kapitel 7 durch den Vergleich verschiedener Finanzierungsformen erläutert. Diese Überlegungen basieren nicht auf einer Untersuchung der Investitionsfähigkeit oder der Fremdkapitelbelastung der Betriebe der Stichprobe. Vor diesem Hintergrund haben diese Überlegungen wiederum einen allgemeinen Charakter und können bei betriebsindividuellen Investitionsentscheidungen als eine Orientierungshilfe berücksichtig werden. 


\subsection{Schlussfolgerungen}

Aus der im Rahmen des Forschungsvorhabens erstellten Arbeit lassen sich einige Schlussfolgerungen und Empfehlungen für die landwirtschaftliche Praxis, die Beratung und die Agrarpolitik ableiten. Im Folgenden werden zunächst die allgemeinen Schlussfolgerungen der gesamten Arbeit, alsdann die Ansatzpunkte und konkrete Empfehlungen für die genannten drei Adressatengruppen formuliert.

\section{Allgemeine Schlussfolgerungen}

In der deutschen Landwirtschaft wurde in den letzten Jahrzehnten ein einschneidender Strukturwandel vollzogen. Markant für diesen Vorgang ist ein kontinuierlicher Rückgang der Anzahl der kleineren Betriebe bei einer gleichzeitigen Zunahme der Anzahl der größeren Einheiten. Der Abwanderung der AK in außerlandwirtschaftliche Bereiche steht die Einführung moderner schlagkräftiger Technik in die landwirtschaftlichen Betriebe gegenüber. Die Tendenz zu weniger und größeren spezialisierten Betrieben wird auch heute fortgesetzt.

Gemessen an der Struktur des deutschen Agrarsektors, der hier als westeuropäischer Standard unterstellt wird, weist die polnische Landwirtschaft wesentliche Defizite auf. Dies zeigt sich v.a. in den Betriebsgrößenstrukturen, der AK-Ausstattung, der Ertragsleistung, der Kapitalverfügbarkeit und nicht zuletzt der rückständigen technischen Ausstattung der landwirtschaftlichen Betriebe Polens.

Die Transformation in der deutschen Landwirtschaft kann als eine aufschlussreiche Orientierungshilfe für die in dem polnischen Agrarsektor erforderlichen Änderungen dienen. Es ist dabei zu bemerken, dass das Szenario nach dem die Transformationsprozesse in der deutschen Landwirtschaft verliefen, unter den polnischen Bedingungen nicht wiederholt werden kann. Dagegen sprechen zum einen die Unterschiede in der Wirtschaftsstruktur in Deutschland in den letzten Jahrzehnten und in der Gegenwart in Polen, zum anderen die veränderten Rahmenbedingungen. Während in Deutschland zu Beginn des in dieser Arbeit betrachteten Zeitraums der Strukturwandel in der Landwirtschaft durch eine Abwanderung der $\mathrm{AK}$ in nichtlandwirtschaftliche Bereiche stark gefördert wurde, kann die aktuelle Wirtschaftslage in Polen und die daraus resultierende Arbeitslosigkeit als ein gewichtiger Hemmungsfaktor der erforderlichen Umwandlungen angesehen werden. Darüber hinaus ist an dieser Stelle zu berücksichtigen, dass der Charakter der Wirtschaftsentwicklung im Laufe der Zeit wesentlichen Änderungen unterliegt. Die Einführung innovativer arbeitsarmer 
Technologien fördert nicht die Steigerung der Beschäftigtenanzahl. Hinzu kommen die zunehmende Wettbewerbsglobalisierung und die Änderung der Agrarpolitik der EU. Unter diesen Bedingungen muss der Strukturwandel einen anderen Charakter haben. Dabei steht außer Zweifel, dass auch in Polen existenzfähige Einheiten entstehen müssen, die auf die Dauer im Wettbewerb bestehen können. Dringend erforderlich sind hier Änderungen in der AK- Ausstattung, der Produktivität und der Mechanisierung der landwirtschaftlichen Betriebe.

Unausweichlich für den weiteren Modernisierungsprozess ist die Verbesserung der Allokation der vorhandenen Kapazitäten und Steigerung der Faktorentlohnung. Wichtig ist dabei die Aktivierung der endogenen Potenziale bestimmter Regionen. Für die in dieser Arbeit ausgewählten Untersuchungsregionen, zeichnen sich unterschiedliche Wege ab. Für die Woiwodschaft Ermland-Masuren kann von guten Entwicklungsbedingungen für große spezialisierte Betriebe der konventionellen Produktion ausgegangen werden. Für kleinere Betriebe, deren Bestandsaufstockung oder Flächenexpansion enge Grenzen gesetzt sind, kann die natürliche Attraktivität dieser Region eine hohe Bedeutung haben. Neben der niedrigen Industrialisierung bieten die natürlichen Vorzüge (hohe Wald- und Gewässerdichte) günstige Bedingungen für die Entwicklung des Landtourismus. In vielen Betrieben der Region Lublin können hingegen positive Effekte durch eine weitere Produktionsdiversifizierung und Erzeugung von qualitativ hochwertigen Produkten erwirkt werden. Als Beispiel hierzu kann der ökologische Landbau angeführt werden. In diesem Fall wird, im Gegenteil zur konventionelleren Produktion über die Umweltaspekte hinaus ein Vorteil in Steigerung der Arbeitsintensität deutlich. Ausgehend von einer kaufkräftigen Nachfrage an Öko -Produkten, sei es in direkter Umgebung oder gar mit der Bestimmung für den Export, kann der ökologische Landbau für viele Betriebe dieser Region eine wesentliche Bedeutung in weiterer Entwicklung haben.

In den entwicklungsfähigen Betrieben ist eine Erhöhung der Produktivität erforderlich. Diese setzt die Einführung moderner Technik voraus. Der Modernisierungsbedarf ist in der Mehrheit der Betriebe durch einen schlechten Zustand der vorhandenen Maschinen zusätzlich verstärkt. Die Ausstattung mit landwirtschaftlichen Maschinen ist in den letzten Jahren quantitativ zwar angestiegen, es handelt sich dabei jedoch zum großen Teil um die Anschaffung von gebrauchten, oft stark abgenutzten und veralteten Maschinen. Daher kann nicht verkannt werden, dass trotz der Steigerung der Maschinenanzahl der Mechanisierungszustand der Mehrzahl der Betriebe unverändert schlecht bleibt. 
Unter den derzeitigen Voraussetzungen ist ein überwiegender Teil der Betriebe, gemessen an dem aus der Produktionsstruktur resultierenden Leistungsbedarf, quantitativ ausreichend ausgestattet. Bei der Modernisierung lassen sich die Mechanisierungskosten mithilfe des überbetrieblichen Maschineneinsatzes reduzieren. Sie werden allerdings für viele Betriebe nicht geringer als bei der gegenwärtigen alten Technik. Durch die Modernisierung kann jedoch die Arbeitsproduktivität und -qualität verbessert werden. Dies gilt besonders für die Betriebe, in denen ein weiteres Wachstum angestrebt wird.

Davon ausgehend, dass die Einführung moderner Technik durch die Entwicklung der überbetrieblichen Zusammenarbeit erleichtert werden soll, sind die sozialen Aspekte des Prozesses zu bedenken. Unter polnischen Bedingungen kann es besonders schwierig fallen, die einzelpersönlichen Ziele der Landwirte zusammenzuführen und zu bündeln. Die das Individualeigentum wahrende Einstellung der Landwirte und ihre Abneigung gegenüber allen Kooperationsformen ist ein wesentlicher Grund hierfür.

Im Bereich der Maschinennutzung kann der angesprochenen Zielrichtung durch die Herstellung dauerhafter Verbindungen zwischen unterschiedlich ausgestatteten Betrieben Rechnung getragen werden. Wie an anderen Stellen ausführlicher erläutert, erscheint für die Realisation dieser Aufgabe die Entwicklung der Institutionen der Maschinenarbeitsvermittlung nach dem Vorbild der deutschen MR wenig geeignet. Der dabei erforderliche organisatorische Aufwand kann bei Einschätzung der möglichen Vorteile sachlich nicht gerechtfertigt werden. Darüber hinaus sprechen gegen diesen Weg auch die in der jüngsten Vergangenheit misslungenen Versuche der Etablierung der MR in Polen. Ein Modernisierungseffekt kann demgegenüber durch die Zusammenarbeit der Landwirte in Maschinengemeinschaften herbeigeführt werden. Dabei handelt es sich in erster Linie um die Maschinengemeinschaften von zwei oder drei Landwirten, die bestimmte Maschinen gemeinsam kaufen und einsetzen. Darüber hinaus können künftig auch größere Maschinengemeinschaften mit mehreren Beteiligten entstehen. Die der Entwicklung dieser Form der Zusammenarbeit immanenten Schwierigkeiten der sozialen Natur, können durch die Eingliederung der Maschinengemeinschaften in die angesprochenen Erzeugergruppen und Auslagerung der Führungsfunktion an eine neutrale Person erfolgreich gemindert werden.

Für die Modernisierung der Mechanisierung ist eine Weiterentwicklung des Lohnmaschineneinsatzes wichtig. Die Maschinendienstleistung kann zunächst in dem bislang bekannten Rahmen durch die landwirtschaftlichen Betriebe mit freien Maschinenkapazitäten 
verrichtet werden. Bei weiterem Wandel und der damit einhergehenden Spezialisierung der landwirtschaftlichen Betriebe kann die Funktion der Dienstleistungsanbieter durch professionelle gewerbliche Lohnunternehmen erfüllt werden.

\section{Empfehlungen an die landwirtschaftliche Praxis}

Bei der Auswahl künftiger Mechanisierung muss von den Landwirten sorgfältig geprüft werden, welchen Einfluss eine bestimmte Mechanisierungsform auf den Betrieb und seine weitere Entwicklung ausüben kann. Verschiedene Mechanisierungsformen haben unterschiedlichen Arbeits- und Kapitalbedarf. Daraus resultiert auch eine unterschiedliche Wirkung auf die Betriebsorganisation. $\mathrm{Zu}$ bedenken sind hier nicht nur die Kapital- und Kostenersparnisse, sondern auch die Alternativen der Anwendung der vorhandenen oder zugänglichen Mittel, sei es für das Betriebswachstum oder für die Entwicklung in außerlandwirtschaftlichen Bereichen.

Im Fall des Verzichts auf die vollständige Eigenmechanisierung und Gestaltung einer Form der Kooperation müssen außer betriebswirtschaftlichen Gesichtspunkten auch die psychosozialen Eigenschaften der Beteiligten berücksichtigt werden. Daher empfiehlt sich eine schrittweise Entwicklung der Zusammenarbeit, beginnend mit unverbindlichen und relativ einfachen Formen wie z.B. der Flächentausch, die gemeinsame Beschaffung der Betriebsstoffe oder der Absatz der landwirtschaftlichen Erzeugnisse bis hin zu Kooperationen mit Kapitalverflechtung.

Der Einsatz gemeinsamer Maschinen im Rahmen der Maschinengemeinschaften kann eine besondere Bedeutung in den mittleren Betrieben haben, die ein weiteres Betriebswachstum anstreben. Denn in den Maschinengemeinschaften kann zum einen der Kapitalbedarf für die erforderlichen Investitionen gesenkt und zum anderen die erforderliche Maschinenauslastung gewährleistet werden. Nicht ohne Belang ist hier die Optimierung der Verwendung der Produktionskapazitäten. Die Maschinengemeinschaften bieten besondere Vorteile in Kombination, in der einer der Partner die freigesetzten Kapazitäten anderweitig, sei es für inner- oder außerbetriebliche Entwicklung, effizient einsetzten kann und der andere durch den gemeinsamen Maschineneinsatz eine bessere Auslastung der vorhandenen Produktionsfaktoren erzielt.

Bei der Auswahl zweckmäßiger Maschinengrößen ist genau der tatsachliche Leistungsbedarf zu untersuchen. Anstelle der Investitionen in die neuen Maschinen kann die Anschaffung von 
Gebrauchtmaschinen aus den westlichen Nachbarländern vorteilhaft werden. Bereits heute besteht beispielsweise ein lebhafter Handel mit Gebrauchtmaschinen zwischen Deutschland und Polen. Bei der Anschaffung dieser Maschinen von Bedeutung ist neben der Verkleinerung des Kapitalbedarfs für die Investitionen auch die Möglichkeit der Senkung der weiteren Kosten des Maschineneinsatzes. Der Anwendung der Gebrauchtmaschinen darf jedoch nicht zu Lasten der Termingerechtheit oder der Arbeitsqualität fallen. Daher empfiehlt es sich, bei den geplanten Investitionen nicht die Maschinen zu bevorzugen, die am billigsten sind, sondern solche, die unter den vorgegebenen Rahmenbedingungen ihre Funktion am besten erfüllen. Es ist hier ein großer Wert auf die Ermittlung optimaler Nutzungsdauer und die Sicherung einer angemessenen Wechselhäufigkeit der Maschinen zu legen.

Der Einsatz eigener Maschinen im Rahmen der Dienstleistung kann wesentliche Vorteile zunächst für die größeren Betriebe bieten. Denn es lassen sich durch die Auslastungssteigerung die Mechanisierungskosten in diesen Betrieben reduzieren. Eine Bedeutung kann hier auch der Verbesserung der AK-Verwendung zukommen. Es müssen an dieser Stelle jedoch die Vor- und Nachteile der zusätzlichen Maschinenauslastung ausgewogen werden. Neben dem Aspekt der möglicherweise veränderten Nutzungsdauer der Maschinen ist hier der Einfluss der zusätzlichen Maschinenauslastung auf den Arbeitsablauf und weitere Entwicklung im eigenen Betrieb zu bedenken.

Die Entwicklung des Lohnmaschineneinsatzes zu einem neuen Betriebszweig kann besondere Bedeutung für kleinere Betriebe haben, in denen keine alternativen rentableren Verwendungschancen des Kapitals oder der eigenen Arbeit vorhanden sind. Die Effekte sind am größten bei Spezialmaschinen mit denen sowohl im Hinblick auf die Leistungsfähigkeit als auch auf die Qualität eine bessere Arbeit verrichtet werden kann als es mit der Anwendung der, in der Mehrheit der landwirtschaftlichen Betriebe vorhandenen Technik möglich wäre. Die angesprochenen Aspekte der Termingerechtheit, der Zuverlässigkeit und somit der optimalen Ersatzstrategie der Maschinen gewinnen hier verstärkt an Bedeutung.

In vielen Betrieben können positive Effekte durch den Verzicht auf eigene Maschinen und Auslagerung der Arbeiten an Dritte erzielt werden. Die Herstellung dauerhafter Zusammenarbeit zwischen den Auftragnehmern und -gebern leistet einen wesentlichen Beitrag nicht nur zur Erhöhung der Arbeitsproduktivität und Steigerung der Wettbewerbsfähigkeit, sondern auch zur Verbesserung der sozialen Bedingungen der Beteiligten. 


\section{Empfehlungen an die Beratung}

Eine zentrale Bedeutung in der Entwicklung der überbetrieblichen Zusammenarbeit wird der landwirtschaftlichen Beratung zukommen. Diese Institution hat diesen Prozess einzuleiten und dauernd $\mathrm{zu}$ begleiten. Wichtig ist hier die Auswahl von geeigneten Kommunikationsmitteln wie die Publikationen in Fachzeitschriften oder die Organisation von Seminaren, Informationsveranstaltungen und Maschinenvorführungen. Nicht zu unterschätzen ist an dieser Stelle das aktive Zugehen der Berater auf den landwirtschaftlichen Betrieb. Aufgrund der Kenntnisse der betriebsindividuellen Entwicklungsbedingungen können die Berater vor Ort die Entwicklung der überbetrieblichen Zusammenarbeit am besten unterstützen und betreuen. Durch den direkten Kontakt mit den Landwirten haben die Berater die Möglichkeit zum optimalen Zeitpunkt, also vor den anstehenden Ersatzinvestitionen fördernd einzugreifen und die konkreten Vor- oder Nachteile bestimmter Mechanisierungsformen aufzuzeigen. Neben der Kostensenkung soll hier der Aspekt der Auswirkung bestimmter Kooperationsformen auf die Betriebsentwicklung gewürdigt werden. Die Aufgabe der Berater ist es aufzuzeigen, dass eine optimale Mechanisierungsform mit Eigentum nur der notwendigen Maschinen und Auslagerung oder Erledigung bestimmter Arbeiten im Rahmen einer Maschinengemeinschaft eine höhere Dynamik der Betriebsentwicklung herbeiführen kann. Dies gründet sich darin, dass auf diese Weise Voraussetzungen geschaffen werden die Arbeit und das Kapital effizienter zu verwenden.

Bei der Auswahl geeigneter Beratungsmittel und Propagierung der Kopperationsidee darf nicht vergessen werden, dass bei den Betriebsentscheidungen nicht allein die Aufklärung der betriebswirtschaftlichen Vorteile, sondern auch die sozialen Erwägungen eine wesentliche Rolle spielen. Diesen Aspekten muss in der Tätigkeit der Berater angemessen Rechnung getragen werden.

Des Weiteren unausweichlich für die Verbreitung der Kooperationsidee ist die Gewinnung der Akzeptanz von innovativ denkenden Landwirten, die eine Führungsrolle in ihrem Umfeld übernehmen und als Multiplikator weiterer Entwicklung agieren können. Wichtig für den Prozess der Propagierung verschiedner Formen der Zusammenarbeit ist auch die Einbeziehung neutraler Einheiten. Dabei handelt es sich zum einen um die bisher erfolgreich kooperierenden Landwirte, zum anderen um die Forschungsanstalten und die Wissenschaft. Neben dem Erfahrungstransfer wichtig ist hier, dies betrifft v.a. die letztgenannten Einrichtungen, die Verbreitung des Fachwissens über moderne Produktionsverfahren und die 
Möglichkeit einer objektiven Beurteilung der politischen oder der durch die Beratungsstellen regional eingeleiteten Förderungsmaßnahmen. Einen wesentlichen Beitrag zu Steigerung der Beratungseffektivität in diesem Bereich kann auch die Einsicht in die institutionellen und wirtschaftlichen Verhältnisse der Nachbarländer und der auf dieser Grundlage aufgebaute Informationsaustausch leisten.

\section{Empfehlungen an die Agrarpolitik}

Eine wesentliche Bedeutung in der Entwicklung der überbetrieblichen Zusammenarbeit in Polen wird der Regierung und den Institutionen des landwirtschaftlichen Umfelds zukommen. Dabei handelt es sich zunächst um die finanzielle Förderung der Kooperationsformen. Diese Förderung ist in der Anfangsphase grundsätzlich zu begrüßen. Sie kann z.B. in Form von Zuschüssen für die Kosten des organisatorischen Aufwandes oder die Gewährung von höheren Fördersätzen für die Investitionen als in den Einzelbetrieben erfolgen. Die Förderung soll jedoch nicht über den Rahmen einer Anlaufhilfe hinausgehen und auf Dauer angelegt werden. Die im ersten Teil der Arbeit dargestellten Ergebnisse zeigen, wie different sich verschiedene Kooperationsformen unter regional unterschiedlichen Rahmenbedingungen entwickeln. Es ist dabei deutlich, dass es keine Form der Zusammenarbeit gibt, die per se die beste ist. Daher soll keiner Form der Zusammenarbeit ein besonderer Rang beigemessen werden. Die einseitige Förderung einer bestimmten Mechanisierungsform, sei es der Eigenmechanisierung oder einer konkreter Form der überbetrieblichen Zusammenarbeit, kann falsche Signale setzten und zu Fehlentscheidungen verleiten.

Wichtig für die Entwicklung der Idee der Kooperation ist die organisatorische Hilfe und die Anpassung des Förderungsprogramms an die regional unterschiedlichen Ausgangsbedingungen. Es ist dabei von Bedeutung, dass die möglichen Interdependenzen zwischen der Landwirtschaft und anderen Sektoren der Volkswirtschaft sowie die daraus resultierenden Entwicklungschancen der landwirtschaftlichen Betriebe genau erwogen werden.

Es ist dringend erforderlich, in das Förderungsprogramm die landwirtschaftliche Beratung einzubeziehen. Denn nur auf diese Weise kann die Richtigkeit der eingeleiteten Maßnahmen gewährleistet werden. Die landwirtschaftlichen Berater können durch Schulungen, Informationsveranstaltungen und direkten Kontakt zu Landwirten nicht nur die Vorteile der Zusammenarbeit aufzeigen, sondern auch den Prozess der Entwicklung bestimmter Zusammenschlüsse dauernd betreuen und helfen, Fehlentscheidungen vermeiden. Dazu sind 
aber weitgehende Änderungen in dem System der landwirtschaftlichen Offizialberatung erforderlich. Angesichts der fortschreitenden Entwicklung im technischen, biologischen und organisatorischen Bereich kann ein universeller Berater keine qualitativ hochwertige Dienstleistung erbringen. Notwendig ist hier eine weitere Differenzierung des Leistungsangebots. Eine wichtige Herausforderung ist auch die Entwicklung der professionellen Beratung im Ökonomiebereich. Weiterhin $\mathrm{zu}$ bedenken ist neben der Einführung der Systeme für die Evaluierung der Leistung der Berater (z.B. durch die Gewährung der Zuschüsse für die Landwirte für die Inanspruchnahme der landwirtschaftlichen Beratung) auch die Unterstützung der Entwicklung der privaten Beratungseinheiten.

Die Förderung der Mechanisierung nicht isoliert betrachtet werden, sondern ist vielmehr als ein integrierter Bestandteil der Entwicklungspolitik der Landwirtschaft zu gestalten. Daher wichtig ist die Schaffung der Bedingungen, unter denen der Einsatz moderner Technik erleichtert wird. Es handelt sich hier namentlich um die Optimierung der Bodenordungsverfahren mit dem Ziel die Struktur der Schläge zu verbessern. Nicht minder wichtig ist an dieser Stelle eine sinnvolle Gestaltung der Förderung der Investitionen in den landwirtschaftlichen Betrieben. Es müssen besonders für die wachstumsfähigen Betriebe die Voraussetzungen geschaffen werden, umfangreiche Investitionen vornehmen zu können. Die von der ARiMR eingeleiteten Programme der finanziellen Förderung der landwirtschaftlichen Investitionen sind grundsätzlich zu befürworten. Es ist aber generell von einer isolierten Förderung der Maschineninvestitionen abzuraten. Die Bezuschussung der Maschineninvestitionen ist nur soweit sinnvoll, als die Investitionen offensichtlich zur Rationalisierung des landwirtschaftlichen Betriebes beitragen und dessen Wirtschaftlichkeit nachhaltig verbessern helfen. In der Förderungspolitik sollten weiterhin noch stärker die Aspekte der weiteren Betriebsentwicklung berücksichtigt werden. Dabei ist die Weiterentwicklung der Buchführung notwendig. In diesem Sinne sind Maßnahmen zu vermeiden, die die Investitionsanreize für Betriebe bilden, die über keine ausreichende Entwicklungsgrundlagen verfügen. Der mehr selektive Charakter der Förderung muss nicht im Widerspruch zum Ziel der Förderung und Erhalt der kleineren Familienbetriebe stehen. Es gilt hier, die Voraussetzungen für die Einbeziehung der kleineren Betriebe in den Kooperationsprozess und dadurch zur Verbesserung der Faktorallokation zu schaffen. 


\section{Zusammenfassung}

In der polnischen Landwirtschaft, die jahrelang unter den Bedingungen der zentralgesteuerten Wirtschaft funktionierte, ist ein akuter Modernisierungsbedarf deutlich. Ein von Bereichen, in denen einschneidige Änderungen nötig sind, ist die Mechanisierung der landwirtschaftlichen Betriebe. Die Möglichkeiten der Einführung moderner schlagkräftiger Technik sind in der Mehrzahl der landwirtschaftlichen Betriebe stark begrenzt. Neben niedriger Kapitalausstattung wirken sich hier auch die beschränkten Möglichkeiten der Sicherung der erforderlichen Auslastung der Maschinenkapazitäten determinierend aus. Diesen Problemen kann unter veränderten Bedingungen im Rahmen der überbetrieblichen Zusammenarbeit begegnet werden. Vor dem Hintergrund dieser These ist das primäre Ziel der vorliegenden Arbeit die Analyse der Entwicklungschancen verschiedener Kooperationsformen in den landwirtschaftlichen Betrieben Polens. Eine besondere Bedeutung haben in diesem Zusammenhang die Erfahrungen mit verschiednen Formen des überbetrieblichen Maschineneinsatzes in der westdeutschen Landwirtschaft. Diese können als wesentliche Orientierungshilfe für den Modernisierungsvorgang der Mechanisierung der polnischen Landwirtschaft dienen.

Der Ausgangspunkt der Überlegungen dieser Arbeit ist die Analyse der Besonderheiten des deutschen Agrarsektors. So befasst sich zunächst das Kapitel 2 mit dem Entwicklungsprozess der westdeutschen Landwirtschaft in den letzten Jahrzehnten bis hin zur Gegenwart. Nur mit dem Grundwissen über den landwirtschaftlichen Strukturwandel kann der vielfältige Zusammenhang der Entwicklung verschiedener Kooperationsformen mit den sich verändernden Rahmenbedingungen der Landwirtschaft und anderen Sektoren der Volkswirtschaft interpretiert werden. Daher werden in diesem Kapitel die wichtigsten Merkmale des Strukturwandels von den Betriebsgrößenstrukturen, über die sozioökonomischen Funktionen der landwirtschaftlichen Betriebe bis hin zur Änderung ihrer technischen Ausstattung beleuchtet. Es wird hier zusätzlich kurz der Einfluss der Politik auf den Modernisierungsprozess durch die direkte und indirekte Investitionsförderung geschildert. Diese Betrachtungen werden auch um einige Aspekte der Landwirtschaftsstruktur der östlichen Bundesländer bereichert. Die Analyse der Landwirtschaft dieser Regionen liefert zwar relativ wenig Erkenntnisse für das primäre Ziel dieser Arbeit, sie trägt aber zum besseren Verständnis der aktuellen Bedeutung und weiteren Entwicklungschancen bestimmter Kooperationsformen unter unterschiedlichen Rahmenbedingungen bei. 
In Kapitel 3 wird der Schwerpunkt auf die Entwicklung verschiedener Kooperationsformen in der deutschen Landwirtschaft gerichtet. Dabei erfolgt zunächst eine kurze Charakteristik aller praxisrelevanten Formen der Zusammenarbeit. In einem weiteren Schritt wird der Rechtsrahmen für die kooperativen Zusammenschlüsse für den gemeinsamen Maschineneinsatz skizziert. In dem darauf folgenden Teil werden die Entstehung, die Entwicklung und die aktuelle Bedeutung der dem Thema dieser Arbeit immanenten Formen der Zusammenarbeit von Nachbarschaftshilfe bis zur Inanspruchnahme der Dienstleistung der professionellen LU analysiert. Diese Studie basiert auf der Auswertung einschlägiger Literatur. Um die dabei gewonnenen Erkenntnisse $\mathrm{zu}$ vervollständigen, wurde eine Expertenbefragung durchgeführt. Das Expertenforum wurde mit der Voraussetzung gestaltet, die Entwicklung verschiedener Kooperationsformen und die daraus resultierenden Vor- und Nachteile sowie evtl. Fehlentscheidungen auf einer möglichst breiten Basis des Fachwissens $\mathrm{zu}$ diskutieren. So besteht das Expertenforum aus den Vertretern der landwirtschaftlichen Praxis, der MR, der LU, der landwirtschaftlichen Beratung und der Wissenschaft. Die Personen wurden v.a. nach der Entwicklung verschiedner Kooperationsformen in Deutschland befragt. Darüber hinaus wurden an die Experten die Fragen nach der, aus ihrer Sicht optimalen Entwicklung bestimmter Kooperationsformen in Polen gerichtet. Die hierzu getroffenen Aussagen werden im weiteren Teil der Arbeit bei der Formulierung der Vorschläge bezüglich der weiteren Mechanisierung in den landwirtschaftlichen Betrieben Polens berücksichtigt.

Aufgrund der durch die Literaturstudie und die Expertenbefragung gewonnenen Erkenntnisse konnte gezeigt werden, wie unterschiedlich sich bestimmte Kooperationsformen vor dem Hintergrund der regionalen und betriebsstrukturellen Bestimmungsgründen entwickeln. Dabei sind wesentliche Änderungen in der Zielrichtung verschiedener Kooperationsformen deutlich. Daraus resultiert auch, unter Berücksichtigung der veränderten Rahmenbedingungen, die aktuelle Bedeutung und die Chancen weiterer Entwicklung des überbetrieblichen Maschineneinsatzes.

Nachdem, der der deutschen Landwirtschaft gewidmete Teil abgeschlossen ist, wird in Kapitel 4 die Struktur des polnischen Agrarsektors geschildert. Dies geschieht mit der Absicht, ähnlich wie in den vorangegangenen Ausführungen, die Rahmenbedingungen für die Entwicklung verschiedener Kooperationsformen in der polnischen Landwirtschaft aufzuzeigen. Ein weiterer Grund für diese Ausführungen war zu zeigen, wo der Verbesserungsbedarf in der polnischen Landwirtschaft liegt. Der Bedarf ist besonders 
deutlich bei Gegenüberstellung der aktuellen Lage des polnischen Agrarsektors und der Landwirtschaftsstruktur Deutschlands. Wenngleich ein direkter Vergleich allein aufgrund unterschiedlicher politischer und wirtschaftlicher Rahmenbedingungen der letzten Jahrzehnte nicht möglich ist, helfen die in den Kapiteln 4 du 2 dargestellten Ergebnisse, die Herausforderungen, vor denen die polnische Landwirtschaft steht, veranschaulichen.

Für die Analyse der Entwicklungschancen bestimmter Kooperationsformen unter polnischen Bedingungen wurde eine Befragung in ausgewählten landwirtschaftlichen Betrieben in zwei unterschiedlich strukturierten Untersuchungsregionen durchgeführt. Die erste Region ist die im nördlichen Teil Polens gelegene Woiwodschaft Ermland-Masuren, die zweite demgegenüber die südöstliche Woiwodschaft Lublin. In der Zusammenarbeit mit den landwirtschaftlichen Beratern aus den örtlichen Beratungsstellen in Olsztyn und Końskowola sind zunächst typische Betriebe ausgewählt und anschließend, anhand eines standardisierten Fragebogens untersucht worden. In die Stichproben sind 29 in Ermland - Masuren bzw. 27 Betriebe in Lublin gefasst. Anhand der erhobenen Daten konnten in Kapitel 5 die aktuelle Mechanisierungsstruktur, der Zustand der vorhandenen Maschinen, die Entwicklungspläne der Betriebsleiter und ihre Einstellung $\mathrm{zu}$ verschiedenen Formen der überbetrieblichen Zusammenarbeit dargestellt werden.

Aufgrund der gewonnenen Erkenntnisse werden in Kapitel 6 die Vorschläge für eine künftige Mechanisierung formuliert. Dabei wird zunächst die Auswahl geeigneter Kooperationsformen erörtert. Unter Berücksichtigung des Kooperationspotenzials werden in einem weiteren Schritt im Hinblick auf bestimmte Maschinen exemplarische Kostenkalkulationen der gemeinsamen Mechanisierung dargestellt. Für die Sicherung des Praxisbezuges der dargestellten Ergebnisse wurde auf das Fachwissen der Experten vor Ort in einer erneuten mündlichen Befragung zurückgegriffen. Die Beschreibung der diesbezüglichen Erkenntnisse schließt dieses Kapitel ab.

In Kapitel 7 werden zunächst die Finanzierungsformen der Maschineninvestitionen aufgezeigt. Neben den Kapitalmarktdarlehen und zinsverbilligten Darlehen aus dem Förderungsprogramm des MRiRW wird hier auch das Leasing beleuchtet. Die Vorzüglichkeit der Finanzierungsformen wird zunächst anhand eines kalkulatorischen Beispiels, dann deskriptiv diskutiert. Darüber hinaus wird in diesem Kapitel auch die Methodik zur Ermittlung der optimalen Ersatzstrategie und ihre praktische Anwendung, anhand eines Schleppers aus den vorangegangenen Kalkulationen skizziert. 
In Kapitel 8, das diese Arbeit abschließt, werden allgemeine Schlussfolgerungen, dann konkrete Empfehlungen für drei Adressatengruppen (die landwirtschaftliche Praxis, die Beratung und die Agrarpolitik) formuliert. Diese Schlussfolgerungen haben einen allgemeinen Charakter und können über die untersuchten Gebiete hinaus auch für andere Regionen als eine praktikable Orientierungshilfe herangezogen werden. 


\section{Quellenverzeichnis}

ANR (2005) Raport działalności. Warszawa 2005.

ARiMR (2005) Przewodnik - Grupy producentów rolnych. Warszawa 2005.

Block, H.-J. (1977) Analyse und Beurteilung der Investitionshilfen des Einzelbetrieblichen Förderungsprogramms für die Landwirtschaft. In Berichte über Landwirtschaft 1977/1978 Band 55.

BML (1987) Rahmenplan der Gemeinschaftaufgabe „Verbesserung der Agrarstruktur und des Küstenschutzes“ für den Zeitraum 1987 bis 1990. Unterrichtung durch die Bundesregierung 12.01.1987.

BML (1974) Rahmenplan der Gemeinschaftaufgabe „Verbesserung der Agrarstruktur und des Küstenschutzes“ für den Zeitraum 1974 bis 1977. Unterrichtung durch die Bundesregierung 16.01.1974.

BMELV (2006) Agrarpolitischer Bericht der Bundesregierung 2006.

BMR e.V.(2004) Jahresbericht.

BMR e.V. (2004) unveröffentlichte Daten zu Gesamtumsatz, Betriebshilfe, und Maschinenvermittlung in den deutschen Maschinenringen. Neuburg an der Donau 24.02.2005

Brandes, W.; Odening M. (1992) Investition, Finanzierung und Wachstum in der Landwirtschaft. Verlag Eugen Ulmer, Stuttgart 1992.

Bundel, B. et al. (1989) Formen der zwischenbetrieblichen Zusammenarbeit in der Landwirtschaft. Aktuelle Empfehlungen, Checklisten und Vertragsbeispiele. DLGArbeitsunterlagen 1989.

Crönlein, J. (1980) Die Förderung von Maschinenringen und Maschinengemeinschaften. In Überbetriebliche Maschinenverwendung in der Landwirtschaft. KTBL-Schrift 244. Landwirtschaftsverlag, Münster 1980.

Crönlein, J. (1979) Förderung von Kooperationen im Produktionsbereich der Landwirtschaft in der Bundesrepublik Deutschland. Darstellung und Analyse der Ziele, Maßnahmen und Ergebnisse öffentlicher Förderung von Kooperationen. Dissertation, Giessen 1979.

Doluschitz, R.; Schwenninger, R. (2003) Nebenerwerbslandwirtschaft. Verlag Eugen Ulmer, Stuttgart 2003.

Doluschitz, R. (2001) Kooperation in der Landwirtschaft. In Berichte über Landwirtschaft S. 375-398, Band 79 März 2001. Landwirtschaftsverlag, Münster 2001.

DWD (2004) Klimastatusbericht des deutschen Wetterdienstes DWD. 
Fasterding, F.; Rixen D. (2005) Analyse der Beschäftigungsmöglichkeiten im Agrarsektor Deutschlands und Beschäftigungseffekte agrarpolitischer Maßnahmen. Bundesforschungsanstalt für Landwirtschaft, Braunschweig 2005.

Gablers Wirtschaftslexikon (1980) Herausgegeben von Sellien, R.; Sellien, H. 10. Auflage. Verlag Gabler GmbH, Wiesbaden 1980.

Geiersberger, E. (2002) Die dritte Bauernbefreiung. Aktualisierter und erweiterter Nachdruck. Österreichisches Kuratorium für Landtechnik und Landentwicklung, Wien 2000.

Grimm, A. et al. (1993) Der Maschinenring der Zukunft: organisierte Nachbarschaftshilfe und ländliches Dienstleistung- Unternehmen. In MR Management\&Technik 4/1993.

Grimm, A. (1980) Gründe und Ergebnis regional unterschiedlicher Entwicklung der überbetrieblichen Maschinenverwendung. In überbetriebliche Maschinenverwendung in der Landwirtschaft KTBL- Schrift 244, Darmstadt 1980.

Grimm, C. (2004) Agrarrecht. 2. Auflage. Verlag C.H.Beck, München 2004.

Grimm, C. (1995) Agrarrecht. 1. Auflage. Verlag C.H.Beck, München 1995.

GUS (2005) Mały rocznik statystyczny. Warszawa 2005.

GUS (2004) Olsztyn Wybrane cechy charakteryzujące zmiany w gospodarstwach indywidualnych. Urząd Statystyczny w Olsztynie 2004.

GUS (2003) Wyniki powszechnego spisu rolnego 2002. Warszawa 2003.

GUS (2002) Biuletyn statystyczny. Warszawa 2004.

Hegenbarth, S. (1977) Kooperationsformen in der polnischen Landwirtschaft. Osteuropastudien der Hochschulen des Landes Hessen Reihe I. Zentrum für kontinentale Agrar- und Wirtschaftsforschung der Justus-Liebig-Universität, Giessen 1977.

Hegenbarth, S. (1973) Die landwirtschaftlichen Zirkel in Polen. Osteuropa 10/1973.

IERiGZ (2006) Rynek środków produkcji i usług dla rolnicta. NR 29, Warszawa 2006.

Isermeyer, H. (1981) Überbetriebliche Maschinenverwendung und wo sie hinpaßt. KTBL Schrift 268. Landwirtschaftsverlag, Münster 1981.

Isermeyer, H. G. (1967) Förderung der überbetrieblichen Maschinenverwendung durch Bund und Länder. In Landtechnik Heft 22, 1967, S.694.

Klare, K.; Doll H. (1996) Stand und Entwicklung der Pachtpreise für landwirtschaftliche Flächen und Betriebe in verschiedenen Gebieten Deutschlands. In Aktuelle Aspekte der Pacht S. 61-129, Schriftenreihe des Hauptverbandes der landwirtschaftlichen Buchstellen und Sachverständigen e.V., Heft 149 Vortragsreihe 13-14. November 1996, Göttingen.

Köhne, M. (2002) Kooperationen mit Zukunft. In Zuckerrüben- Zeitung, Heft 1 S. 12-13, Januar 2002. 
Köhne, M. (2000) Landwirtschaftliche Taxationslehre. 3. Auflage. Parey Buchverlag Blackwell Wissenschaft- Verlag, Berlin, Wien 2000.

Köhne, M.; Wesche, R. (1995) Landwirtschaftliche Steuerlehre. 3. Auflage. Verlag Eugen Ulmer, Stuttgart 1995.

Köhne, M.; Wesche, R. (1982) Die Besteuerung der Landwirtschaft. Verlag Eugen Ulmer, Stuttgart 1982.

Köhne, M. (1981) Maschinen pachten statt kaufen- Vorsicht bei falschen Vergleichen. In DLG- Mitteilungen 1981, Heft 6, S. 310-314.

Köhne, M. (1980) Zur optimalen Ersatzstrategie bei Landmaschinen unter Berücksichtigung von Inflation und Steuern. In Agrarwirtschaft Heft 9, S. 257-266. Alfred Strothe Verlag, Hannover 1980.

Krüll, H. (1990) Räumliche Differenzierung der Landwirtschaft in der Bundesrepublik Deutschland. Forschungsgesellschaft für Agrarpolitik und Agrarsoziologie e.V., Bonn 1990.

Kübler, F. (1994) Gesellschaftsrecht. 4. Auflage. Müller Juristischer Verlag, Heidelberg 1994.

Link, H. (1995) Kooperationen in der landwirtschaftlichen Produktion. Eugen Ulmer Verlag, Stuttgart 1995.

Linnemann, O. (2001) Simulation von Entwicklungspfaden landwirtschaftlicher Betriebe in Nordwestpolen unter Berücksichtigung von Transformation und bevorstehendem EU-Beitritt. In Agrarwirtschaft Sonderheft 171. Verlag Agrimedia, Bergen/Dumme 2001.

Lischka, G. (1996) Rentabilität in Ackerbau und Milchviehhaltung- Modellkalkulation. Erfolgreich investieren in der Landwirtschaft Polens. Unveröffentlichter Beitrag zum DLG Länderseminar 25.04.1996, Göttingen.

Lorencowicz, E. (1997) Ocena kosztów mechanizacji. Problemy metodyczne. In Informatyka, zarządzanie i technika rolnicza w procesach dostosowawczych gospodarki żywnościowej. Bydgosz - Ciechocinek 1997.

Mann, K.; Muziol, O. (2001) Darstellung erfolgreicher Kooperationen und Analyse der Erfolgsfaktoren. In Betriebsgesellschaft in der Landwirtschaft. Chancen, Grenzen im Strukturwandel. Rentenbank, Schriftenreihe Band 15, S. 55-97, Frankfurt am Main 2001.

Martowski, A.-S.; Kośmider M. (2004) Przewodnik po leasingu w Polsce. Fundacja rozwoju rachunkowości, Warszawa 2004.

Milch, W. (1982) Einfluß der Besteuerung auf die optimale Nutzungsdauer von Maschinen. In Agrarwirtschaft 1982, Heft 10, S. 309-319. Alfred Strothe Verlag, Hannover 1980.

MRiRW (2006) unveröffentlichte Daten über Erzeugergruppen 10.03.2006.

MRiRW (2003) Rolnictwo i gospodarka żywnościowa w Polsce w aspekcie intergracji z EU. Warszawa 2003. 
Muzalewski, A. (2005) Kredytowanie zespołów i usług maszynowych. In Technika Rolnicza, Ogrodnicza i Leśna 11/2005.

Muzalewski, A. (2005) unveröffentlichter Bericht zur technischen Ausstattung der polnischen Landwirtschaft 10.05.2005.

Muzalewski, A. (2004) Dobór maszyn, koszty mechanizacji i efekty modernizacji gospodarstw rolniczych w latach 1996-2030 (stan i perspektywy)- ekspertyza IBMER Nr. XXXVII/345, Warszawa 2004.

Muzalewski, A. (2003) Zespołowe i usługowe użytkowanie maszyn. In Aktualnosci Techniki Rolniczej Nr.8, Warszawa 2003.

Olszewski, T.; Muzalewski, A. (1999) Maschinenringe in Polen- aktueller Stand Probleme und Entwicklungsperspektiven. Unveröffentlichter Beitrag zur Tagung der deutschen Maschinenringe 1999.

Ostrowski, L. et al (2000) Rynek ziemi rolniczej. Stan i perspektywy. IERiGZ, Warszawa 2000 .

Reimers, T. (1996) Ein Beitrag zur Investitionsrechnung für Landmaschinen. Wissenschaftsverlag Vauk Kiel KG, Kiel 1996

Reisch, E.; Zeddies J. (1977) Einführung in die landwirtschaftliche Betriebslehre. Band 2. Spezieller Teil. Grundlagen und Methoden der Entscheidung, Ökonomik der tierischen Produktion. Verlag Eugen Ulmer, Stuttgart 1977.

Riemann, A. (1980) Gründe und Ergebnis regional unterschiedlicher Entwicklungen überbetrieblicher Maschinenverwendung. Die Verhältnisse in Nordrhein-Westfalen. In Überbetriebliche Maschinenverwendung in der Landwirtschaft, S. 29-33 KTBL-Schrift 244. Landwirtschaftsverlag, Münster 1980.

Schaefer-Kehnert W. (1963) Die Kosten des Landmaschineneinsatzes. Berichte über Landtechnik. Herausgegeben vom Kuratorium für die Technik in der Landwirtschaft, Frankfurt/Main 1969.

Scheffer, F.; Schachtschabel, P. (2002) Lehrbuch der Bodenkunde. 15. Auflage. Spektrum Akademischer Verlag, Heidelberg 2002.

Schaltegger, S. (1999) Öko-Effizienz als Element des sozio-ökonomisch vernünftigen Umweltmanagements. In Ökologisches Wirtschaften 1999 Ausgabe 3, S. 12-14.

Schmidt, K. (1983) Landwirtschaftliche Lohnunternehmen heute und morgen. KTBL- Schrift 286. Landwirtschaftsverlag, Münster 1983.

Sikorska, A. et al (2004) Rynek ziemi rolniczej. Stan i perspektywy. IERiGZ, Warszawa 2004.

Spittler. H.-J. (2002) Leasing für die Praxis. Fachverlag Deutscher Wirtschaftsdienst GmbH\&Co.KG, Köln 2002. 
Statistisches Bundesamt (2005) Land- und Forst, Fischerei. Bodenfläche nach Art der tatsächlichen Nutzung. Fachserie 3/ Reihe 5.1.

Statistisches Bundesamt (2005) Land- und Forst, Fischerei. Eigentums- und Pachtverhältnisse. Agrarstrukturerhebung 2003. Fachserie 3/ Reihe 2.1.6.

Statistisches Bundesamt (2005) Land- und Forst, Fischerei. Kaufwerte für landwirtschaftliche Grundstücke. Fachserie 3/ Reihe 2.4.

Statistisches Bundesamt (2004) Land- und Forstwirtschaft, Fischerei. Betriebswirtschaftliche Viehbestände und tierische Erzeugung. Fachserie 3/ Reihe 4.

Statistisches Bundesamt (2003) Land- und Forstwirtschaft, Fischerei. Betriebswirtschaftliche Ausrichtung und Standarddeckungsbeiträge. Fachserie 3/ Reihe 2.1.4.

Statistisches Bundesamt (2003) Land- und Forstwirtschaft, Fischerei. Ausgewählte Zahlen für die Agrarwirtschaft. Fachserie 3/ Reihe 2.1.4.

Statistisches Bundesamt (1999) Land- und Forstwirtschaft, Fischerei. Technische Betriebsmittel. Fachserie 3/ Reihe 2.3.

Statistisches Bundesamt (1995) Ausstattung mit und Einsatz von landwirtschaftlichen Maschinen. Fachserie 3/ Reihe 2.1.9.

Szczurowna, A. (2004) Nieruchomości rolne skarbu państwa w dzierżawie. Unveröffentlichte Daten 14.12.2004.

Theuvsen, L. (2003) Kooperation in der Landwirtschaft. Formen, Wirkungen und aktuelle Bedeutung. Diskussionsbeitrag 0303, Institut für Agrarökonomie der Universität in Göttingen.

Wesche, R. (2003) Rechtsformen landwirtschaftlicher Unternehmen. AID Heft 1147/2003, Bonn 2003.

Winkler, W. (1997) Rechtsformen der Kooperation in der Landwirtschaft. AID Heft XXX/1997, Bonn 1997.

Winkler, W. (1996) Entwicklung und Handhabung des deutschen Landpachtrechtes und dessen Zukunft in der EU. In Aktuelle Aspekte der Landpacht, S. 7-59. Schriftenreihe des Hauptverbandes der landwirtschaftlichen Buchstellen und Sachverständigen e.V., Heft 149 Vortragsreihe 13-14. November 1996, Göttingen.

Wüst, S. (2003) Maschinengemeinschaften gründen und erfolgreich führen. KTBL Sonderveröffentlichung 042, Darmstadt 2003. 
Gesetze:

Gesetz über die Umsatzsteuer von 11.03.2004, Dz.U.04.54.535

Gesetz über Entwicklung des ländlichen Raums von 11.04.2003, Dz.U.03.64.592.

Gesetz über landwirtschaftliche Erzeugergruppen, die Liste der Erzeugnisse, die minimalen Umsätze und die Anzahl der Mitglieder der Erzeugergruppen von 4.07.2003, Dz.U.03.138.1319

Gesetz über die Änderung des Gesetzes über die Einkommenstuer von natürlichen und juristischen Personen von 06.09.2001, Dz.U.01.106.1150

Gesetz über die Änderung des Zivilrechts von 26.07.200, Dz.U.00.74.857

Gesetz über landwirtschaftliche Erzeugergruppen und Verbände von 15.09.2000, Dz.U.00.88.9983.

Gesetz über öffentliche Statistik von 26.06.1995, Dz.U.95.88.439 mit späteren Änderungen.

Gesetz zur Förderung der bayerischen Landwirtschaft (LwFöG) von 27.10.1970.

Internetquellen:

MRiRW (2005) Informationen zu Vorzugskrediten zum 20.07.2005 http://www.minrol.gov.pl /FileRepozytory/FileRepozytoryShowImage.aspx?item id=4998 Abrufdatum 03.04.2006

BLU e.V. (2002) Informationen zum Thema Lohunternehmen in Deutschland, http://www.lohnunternehmen.de/index.htm Abrufdatum 24.12.2003.

BMELV (2006) GAK - Rahmenplan http://www.bmelv.de/cln_045/nn_749972/sid 2490B09 0C26B98EA6E959FE55D73A0B1/DE/04Landwirtschaft/Foerderung/GAK/Rahmenplan2006 /Rahmenplan2006.html_nnn=true Abrufdatum 03.05.2006

BMELV (2006) Methodische Erläuterungen zu den Buchführungsergebnissen der Testbetriebe http://www.bmelv.de/cln_044/nn_749972/SharedDocs/downloads/Agrarbericht/ buchfuehrungsergebnisse/methode.html_nnn=true Abrufdatum 12.04.2006

BVVG (2006) Ergebnisse der Aktivitäten http://www.bvvg.de/internet/internet.nsf/vBroInfo/i Ergebnisse?Opendocument Abrufdatum 07.02.2006.

GUS Olsztyn (2006) Statistisches Amt in Olsztyn, allgemeine Informationen über die Woiwodschaft Ermland - Masuren http://www.stat.gov.pl/urzedy/olsz/index.htm Abrufdatum 08.02.2006

GUS Lublin (2006) Statistisches Amt in Lublin, allgemeine Informationen über die Woiwodschaft Lublin http://www.stat.gov.pl/urzedy/lublin/index.htm Abrufdatum 08.02.2006

MR- Mittelholstein (2003) http://www.mr-mittelholstein.de/ Abrufdatum 11.05.2003. 
Pressemitteilung des DWDs (2004) http://www.dwd.de/de/Zusatzmenues/Presse/Mitteilungen 20040505.htm. Abrufdatum 12.07.2004.

Liste der in Deutschland befragten Experten:

Herr Amelung, C. Betriebswirtschaftliches Büro Göttingen 31.01.2005

Frau Eickemeier, E. LWK Hannover 17.01.2005

Herr von Felbert, S. BMR e.V. Neuburg an der Donau 03.02.2005

Herr Grimm, A. ehemals BMR e.V. München 23.02.2005

Herr Grubber, W. Arbeitsgruppe der MR Rheinland - Pfalz 14.02.2005

Herr Hamman, A. MR Leinetal 16.02.2005

Herr Hampe, J. MR Göttingen 28.01.2005

Herr Heitman, G. LWK Hannover 17.01.2005

Herr Henke, J. LU Wagenfeld 08.02.2005

Herr Hennies, H. Praktiker Uelze 02.01.2005

Herr Isermeyer, H. Praktiker 30.03.2005

Herr Kadner, K. KTBL Darmstadt 14.12.2004

Herr Klischat, U. LWK Hannover 17.01.2005

Herr Kowalewski, LWK Weser Ems 09.02.2005

Herr Köhler, K. Praktiker Lehrte 03.01.2005

Herr Lange, J. LVMR Hannover 03.02.2005

Herr Link, H. ehemals Bayerische Landesanstalt für Betriebswirtschaft und Agrarstruktur München 23.02.2005

Herr Mann, C. BMR e.V. Neuburg an der Donau 24.02.2005

Herr Pentzlin, K. BLU e.V. 16.02.2005

Herr Pfadler, W. Stattliche Führungsakademie Landshut 25.02.2005

Herr Schmidt, A. BLU e.V. 17.01.2005

Herr Schneemann, R. LWK Hannover Kreisstelle Göttingen 17.01.2005 
Herr Schweer, C. Praktiker Lehrte 03.01.2005

Herr Traulsen, H. Deula Schleswig Holstein 02.01.2005

Herr Uppenkamp, N. LWK Nordrhein - Westfalen 24.01.2005

Herr Volk, L. Fachhochschule Südwestfalen. Abt. Soest 24.01.2005

\section{Liste der in Polen befragten Experten:}

Herr Banach, W. Zentrale Stelle der landwirtschaftlichen Beratung Lublin, Końskowola 30.03.2006

Herr Bawol, S. Zentrale Stelle der landwirtschaftlichen Beratung Ermland-Masuren, Olsztyn 27.03.2006

Herr Grycko, J. Zentrale Stelle der landwirtschaftlichen Beratung Ermland - Masuren, Olsztyn 27.03.2006

Frau Kamińska, E. Erzeugergruppe, Elbląg 27.03.2006

Frau Kozak, M. MriRW, Warszawa 29.03.2006

Herr Kozlowski, T. CNH Polen, Płock 28.03.2006

Herr Krupinski. J. Zentrale Stelle der landwirtschaftlichen Beratung Ermland - Masuren, Olsztyn 27.03.2006

Herr Lorencowicz, E. Agrarakademie Lublin 30.03.2006

Herr Muzalewski, A. IBMER,Warszawa 29.03.2006

Herr Sobieś, J. LBS Bychawa 30.03.2006

Sonstige persönliche Mitteilungen:

Frau Ahnert, G. Maschinen- und Betriebshilfsring Rochlitz 18.04.2006

Herr Herbeg, S. MR Mittelholstein 10.02.2005

Herr Mann, H.-J. LBB mbH Göttingen 08.05.2006

Frau Pellot, M. KBM e.V. 07.04.2005

Frau Rosenauer, A. BMELV 25.04.2006

Herr Schmidt, A. BLU e.V. 26.04.2006

Herr Walter, J. KBM e.V. 06.05.2005 
Herr Weiershäuser, L. KTBL 19.07.2005

Herr Winkler, W. Universität in Göttingen Institut für Landwirtschaftsrecht 12.10.2004

Frau Zepuntke, A. Statistisches Bundesamt 11.04.2006 


\section{Anhang}

A.1 Zugmaschinen und motorische Zugkraft in der deutschen Landwirtschaft _......... 197

A.2 Ackerschlepperbestand in Deutschland nach Gruppen der Motorleistung _.......... 197

A.3 Auswahlkriterien der Rechtsformen 198

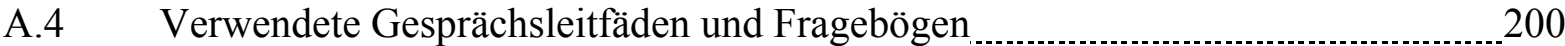

A.4.1 Gesprächsleitfaden für die Expertenbefragung in Deutschland _ _ 200

A.4.2 Fragebogen für die Befragung der Landwirte in Polen _.................................. 202

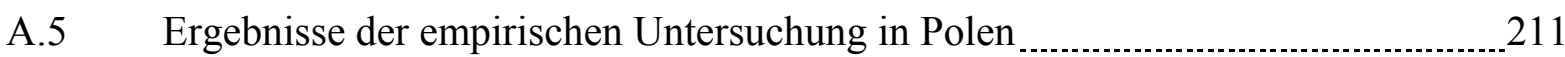

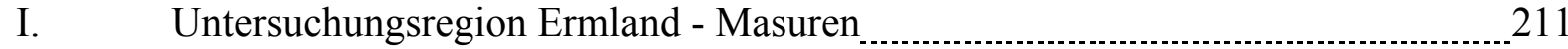

Tabelle 1: Nutzung der LF

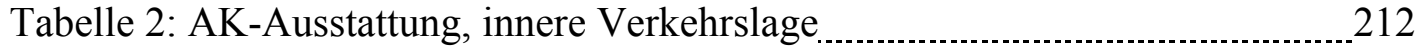

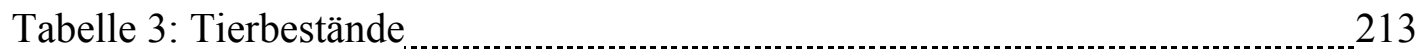

Tabelle 4: Maschinenbesatz

Tabelle 5: Kosten des ersten Schleppers _............................................ 215

Tabelle 6: Kosten des zweiten Schleppers

Tabelle 7: Kosten des dritten Schleppers $\ldots \ldots \ldots$

Tabelle 8: Kostend des vierten Schleppers $\ldots$

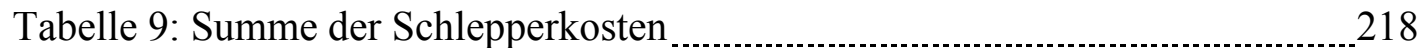

Tabelle 9: Kosten der Mähdrescher 218

II. Untersuchungsregion Lublin

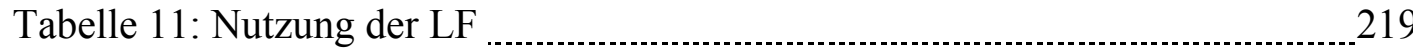

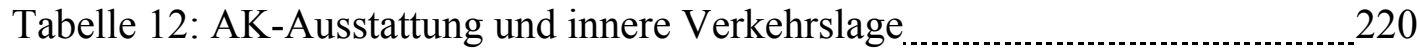

Tabelle 13: Tierbestände

Tabelle 14: Maschinenbesatz

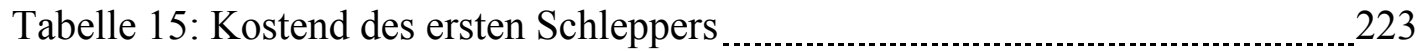

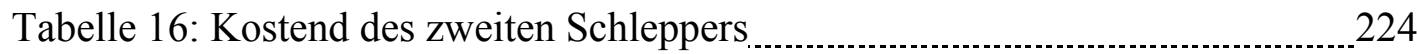

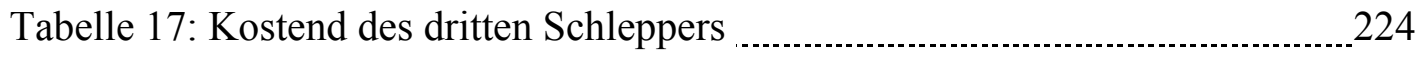

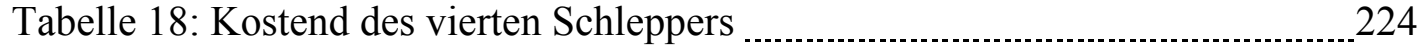

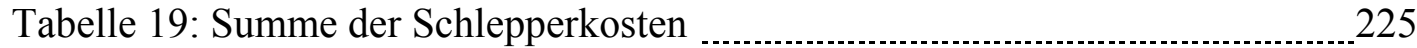

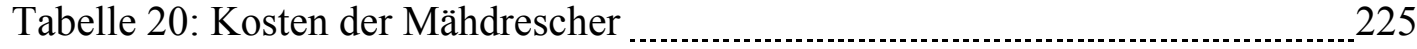


Tabelle A. 1: Zugmaschinen und motorische Zugkraft in der deutschen Landwirtschaft

\begin{tabular}{|l|c|c|c|c|c|}
\hline \multirow{2}{*}{ Jahr } & $\begin{array}{c}\text { Schlepperbestand } \\
\text { Land- und Forstwirtschaft, } \\
\text { Fischerei }\end{array}$ & $\begin{array}{c}\text { darunter } \\
\text { Landwirtschaft }\end{array}$ & \multicolumn{3}{|c|}{$\begin{array}{c}\text { Motorleistung der Schlepper in der } \\
\text { Land- und Forstwirtschaft, Fischerei }\end{array}$} \\
\cline { 2 - 5 } & 1000 & 1000 & $1000 \mathrm{~kW}$ & $\mathrm{~kW}$ & $\mathrm{~kW} / 100 \mathrm{ha} \mathrm{LF}$ \\
\hline 1996 & 1166,5 & 1117,9 & 43315,1 & 37,1 & 249,9 \\
1998 & 1072,2 & 1027,5 & 40585,0 & 37,8 & 233,6 \\
$2000^{1}$ & 948,2 & 938,6 & 32380,0 & 34,5 & 190 \\
2002 & 944,8 & 944,7 & 31873,0 & 33,8 & 187,7 \\
$2004^{2}$ & 869,6 & 868,4 & 37937,0 & 43,6 & 222,9 \\
\hline
\end{tabular}

Quelle: Statistisches Jahrbuch über Ernährung Landwirtschaft und Forsten 2005 nach Kraftfahrt- Bundesamt 2005,

Tabelle A. 2: Ackerschlepperbestand in Deutschland nach Gruppen der Motorleistung

\begin{tabular}{|c|c|c|c|c|c|c|c|}
\hline \multirow{3}{*}{ Jahr } & \multirow{2}{*}{\multicolumn{5}{|c|}{ Schlepperbestand }} & \multirow{3}{*}{ Insgesamt } & \multirow{3}{*}{$\begin{array}{c}\text { Einschl. } \\
\text { Geräteträger und } \\
\text { Straßen- } \\
\text { zugmaschinen }\end{array}$} \\
\hline & & & & & & & \\
\hline & bis 30 & $31-50$ & $51-70$ & $71-90$ & 91 und mehr & & \\
\hline 2001 & 230149 & 392468 & 273086 & 98353 & 78662 & 1072718 & 1770659 \\
\hline 2002 & 236631 & 392081 & 275852 & 100966 & 84854 & 1090384 & 1773193 \\
\hline 2003 & 242900 & 390602 & 277024 & 104704 & 91704 & 1106556 & 1952243 \\
\hline 2004 & 249486 & 389101 & 278078 & 106292 & 96718 & 1119675 & 1952298 \\
\hline $2005^{3}$ & 257286 & 388289 & 279361 & 108224 & 102087 & 1135247 & 1961934 \\
\hline
\end{tabular}

Quelle: Statistisches Jahrbuch über Ernährung Landwirtschaft und Forsten 2005 nach Kraftfahrt- Bundesamt 2005

\footnotetext{
1 Infolge der Änderungen in der Abgrenzung sind die Daten mit denen der Vorjahre nicht vergleichbar.

${ }^{2}$ Bestand einschließlich Ackerschlepper, Geräteträger, Straßen- und Sattelzugmaschinen. Motorleistung wird aus Mittelwerten errechnet.

3 Vorläufig.
} 


\section{A. 3 Auswahlkriterien der Rechtsformen}

Übersicht 1: Auswahlkriterien der Rechtsformen

\section{Personengesellschaften}

von den im Vertrag festgelegten Ausnahmen abgesehen

Haftung einschließlich Privatvermögen bei KG beschränkte,

Haftung der Kommanditisten

2. Kapitalbeschaffung

3. Effizienz der Unternehmensführung

4. Abhängigkeit von anderen Mitgliedern

5. Steuerliche Aspekte

6. Inanspruchnahme von Fördermitteln

7. Entlohnung der eingebrachten Anteile

8. Rücklagenbildung

9. Rechtsformabhängiger Aufwand

(Notarkosten, Eintragungsgebühren,

Publikationspflicht) in der Regel ist kein Gesellschaftsvermögen erforderlich

wegen der gemeinsamer Entscheidung kann bei GbR die

Unternehmensführung erschwert werden, im Falle von $\mathrm{OHG}$

Und KG besteht Einzelnpersonenführung mit Widerspruchsrecht

der anderen Mitglieder

Bestand abhängig von der Entscheidung der Betroffenen

bei GbR kann das Ausscheiden eines Mitglieds zur Beendigung des

Zusammenschlusses führen, Kündigung und Auflösung bei Personengesellschaften

des Handelsrechts ist im Vergleich zu GbR deutlich erschwert

große Vorzüglichkeit wegen der Möglichkeit der separaten Buchführung,

von Ausnahmen abgesehen keine Gewerbesteuerpflicht

potenziell unbeschränkter Zugang zu Fördermitteln

vertragsspezifisch

besteht Möglichkeit der Rücklagenbildung

meistens gering

\section{Mischformen}

entstehen aus Kombination von $\mathrm{GmbH}$ AG und KG im Hinblick auf die Ziele weisen sie Eigenschaften der oben genannten Rechtsformen auf, erlauben

\section{aber die Verfolgung spezifischen} Zwecke 


\section{Körperschaften}

Vereine

1. Haftung

2. Kapitalbeschaffung

3. Effizienz der Unternehmensführung

4. Abhängigkeit von anderen Mitgliedern

\section{Steuerliche Aspekte}

6. Inanspruchnahme von Fördermitteln

7. Entlohnung der eingebrachten Anteile

8. Rücklagenbildung

9. Rechtsformabhängiger Aufwand

(Notarkosten, Eintragungsgebühr,

Publikationspflicht) beschränkt auf Vereinsvermögen

bei nicht rechtsfähigem Verein

haftet der Handelnde persönlich

bei wirtschaftlichem Verein mit Rechtsfähigkei

besteht die Pflicht der Mindestkapitalbeschaffung

Leitung durch den Vorstand der Mitliederversammlung können umfangreiche Entscheidungsrechte eingeräumt werden

Kündigung für Austritt max. 2 Jahre, kein Abfindungsanspruch Mitgliedschaft ist nicht übertragbar und nicht vererblich,

\section{auf Grund der Rechtsform Gewerbesteuerpflicht}

wegen der ideellen Zwecke und beschränkter Haftung ist der Zugang zu Fördermitteln erschwert

keine

besteht Möglichkeit der Rücklagenbildung

meistens gering

\section{Genossenschaften}

Genossenschaftsvermögen

den Genossen können unter Umständen

die Nachschusspflicht auferlegt werden

kein Mindestkapital vorgeschrieben

Abstimmung nach Köpfen, 1 Genosse 1 Stimme

Mehrstimmrechte bis zu 3 Stimmen

Leitung durch den Vorstand

Kündigungsfrist vertraglich festgelegt max. 5 Jahre Übertragung der Anteile, die Existenz ist durch das Ausscheiden eines Mitglieds nicht gefährdet

auf Grund der Rechtsform Gewerbesteuerpflicht

wegen beschränkter Haftung kann der Zugang zu Förderungsmitteln erschwert werden

Verteilung von Gewinn bzw. Verlust nach Geschäftsanteilen

gesetzliche Pflicht der Rücklagenbildung

mindestens 7 Mitglieder, Eintragung in Genossenschaftsregister, muss einem Prüfungsverband beitraten der eine Pflichtprüfung durchführt
Kapitalgesellschaften

beschränkt auf Gesellschaftsvermögen

Stammkapital min. 25.000 EUR( GmbH) Grundkapital 50.000 EUR (AG)

GmbH geleitet vom Geschäftsführe bei AG Leitung durch den Vorstand

ertraglich festgelegte Vereinbarungen

insbesondere bei $\mathrm{GmbH}$, die Geschäftsanteile sind in der Regel vererblich und veräußerlich

auf Grund der Rechtsform Gewerbesteuerpflicht

relativ einfach bei $\mathrm{AG}$, bei $\mathrm{GmbH}$ kann erschwer werden

direkte Entlohnung der Anteile anhand der diesbezüglichen Vereinbarungen

besteht Möglichkeit der Rücklagenbildung

im Vergleich zu anderen Formen ist der

Aufwand hoch 
A. 4 Verwendete Gesprächsleitfäden und Fragebögen

A. 4.1 Gesprächsleitfaden für die Expertenbefragung in Deutschland

\section{Zu Ihrer Person}

1. In welcher Art der Organisation sind sie tätig?

2. Wo liegen Ihre Tätigkeitsschwerpunkte?

\section{Zur Entwicklung der Kooperationsformen in der Region Ihrer Berufstätigkeit}

3. Welche Formen der überbetrieblichen Maschinennutzung sind in Ihrer Region vertreten. Können Sie die Rangfolge der Bedeutung der verschiedenen Formen anhand von relativen Kriterien wie z.B. Umsatzanteil bestimmen?

4. Welche Arbeitsbereiche werden innerhalb dieser Formen erschlossen?

5. Worin liegt die Hauptmotivation bei der Zusammenarbeit im Bereich der Maschinennutzung in den Ihnen bekannten Betrieben?

6. Ist im Laufe der Entwicklung der Zusammenarbeit eine gewisse Anpassung der Betriebsorganisation der Betroffenen zu erkennen?

7. In welchem Maße entsteht die Zusammenarbeit aus der Eigeninitiative der Landwirte und welche Bedeutung kommt an der Stelle den Institutionen des landwirtschaftlichen Umfelds zu?

8. Wie erfolgt die Beratung bezüglich der überbetrieblichen Maschinennutzung?

9. Welche Bereiche bedürfen in der Entwicklung der Zusammenarbeit besonderer Aufmerksamkeit?

10. Wo erkennen Sie besondere Hemmnisse, Probleme und eventuelle Fehlentscheidungen in der Entwicklung der Formen der Zusammenarbeit im Hinblick auf existierende Betriebe?

11. Welche Voraussetzungen müssen für eine erfolgreiche Kooperation erfüllt werden?

\section{Zur allgemeinen Entwicklung der Kooperation im Bereich der Maschinennutzung}

12. Welche Bedeutung kommt nach Ihrer Ansicht heutzutage den unterschiedlichen Formen der überbetrieblichen Maschinennutung zu?

- Nachbarschaftshilfe

- MR

- Maschinengemeinschaft
- Lohnunternehmen

- große Maschinengesellschaften 
13. Wo liegen Ihrer Ansicht nach die Hauptmotive der Entwicklung der unterschiedlichen Formen der überbetrieblichen Maschinennutzung in Deutschland?

14. Wie schätzen Sie die Rolle des landwirtschaftlichen Umfelds in der Förderung der Idee der überbetrieblichen Maschinennutzung ein?

15. Welche Bedeutung dürfte an der Stelle die finanzielle Förderung des Staates haben?

- MR

- Maschinengemeinschaft

- Lohunternehmen

16. Kann man im Hinblick auf den gesamten Mechanisierungsprozess über bestimmte Entwicklungsphasen der Kooperationsformen sprechen?

17. Wo gab es besondere Schwierigkeiten bzw. grobe Fehler in dem gesamten Vorgang?

18. Worin sehen Sie besondere Erfolge der Entwicklung der überbetrieblichen Maschinennutzung?

\section{Fazit}

19. Kann die gesamte Modernisierung der Mechanisierung in der deutschen Landwirtschaft in den letzten Jahren positiv beurteilt werden?

- wo sehen sie eventuelle Defizite und weiteren Verbesserungsbedarf?

20. Wie dürfte sich die Zusammenarbeit im Bereich der Maschinennutzung weiter entwickeln?

21. Wie kann von Ihrem Standpunkt aus die Förderung der überbetrieblichen Maschinennutzung optimal gestaltet werden?

- welche Rolle kann hier der Staat sowie die landwirtschaftliche Praxis selbst spielen?

22. Können Sie sich vorstellen, welche Kooperationsformen bei der Modernisierung am besten für die polnische Landwirtschaft geeignet wären? 
A. 4.2 Fragebogen für die Befragung der Landwirte in Polen

Georg- August-Universität

Institut für Agrarökonomie

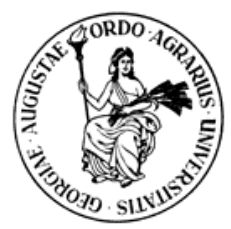

\section{Fragebogen zum Forschungsvorhaben}

„, Die künftige Mechanisierung der polnischen Landwirtschaft unter besonderer

Berücksichtigung der deutschen Erfahrungen mit Kooperationen“"

Name des Befragten bzw. des Unternehmers

Unternehmenssitz

Ort und Datum der Befragung

Telefonnummer/ E-Mail der Kontaktperson

Dieser Fragebogen gliedert sich in fünf Teile. Das Ausfühlen des Fragebogens erfolgt entweder durch Eintragen bestimmter Daten in die Tabellen oder durch schriftliche Beantwortung der gestellten Fragen.

Wir versichern Ihnen, dass alle Daten über Ihren Betrieb vertraulich behandelt werden. Die Daten sollen ausschließlich dem wissenschaftlichen Ziel dieses Forschungsvorhabens dienen. Daher werden sie unter keinen Umständen an Dritte und Nichtbeteiligte weitergegeben. 
I. Angaben zum Betrieb

1. Organisationsform:
$\square$ Einzelunternehmen
$\square$ BGB Gesellschaft
$\square \mathrm{GmbH}$
$\square$

2. Erwerbsform
$\square$ Haupterwerb
$\square$ Nebenerwerb

\begin{tabular}{|l|l|l|l|}
\hline 3. Flächenausstattung & eigene Fläche & \multicolumn{2}{|c|}{ Pachtfläche verpachtet } \\
\hline Ackerland & & & \\
\hline Grünland & & & \\
\hline Wald & & & \\
\hline Sonstige: & & & \\
\hline- & & & \\
\hline
\end{tabular}

\begin{tabular}{|l|l|l|l|l|l|l|}
\hline 4. Bodenqualität \\
\hline Bonitätsklassen & I & II & III & IV & V & VI \\
\hline LF in ha & & & & & & \\
\hline
\end{tabular}

\begin{tabular}{|l|l|}
\hline 5. Verkehrslage & \\
\hline Anzahl der Schläge & \\
\hline Durchschnittliche Schlaggröße & \\
\hline Durchschnittliche Entfernung der Schläge vom Hof & \\
\hline
\end{tabular}

\begin{tabular}{|l|l|}
\hline 6. Gebäude und Anlagen \\
\hline Art & Baujahr, Größe, Bezeichnung \\
\hline Wohngebäude & \\
\hline Wirtschaftsgebäude & \\
\hline Anlagen & \\
\hline
\end{tabular}


II. Produktionsstruktur

\begin{tabular}{|l|l|l|}
\hline 7. Pflanzenproduktion im Durchschnitt der letzten fünf Jahre & \multicolumn{1}{|c|}{ Ertrag in dt/ha } \\
\hline Getreide & Anbaufläche in ha & \\
\hline Roggen & & \\
\hline Weizen & & \\
\hline Gerste & & \\
\hline Triticale & & \\
\hline Mafer & & \\
\hline Hacksfrüchte & & \\
\hline Kartoffeln & & \\
\hline Zuckerrüben & & \\
\hline weitere Früchte & & \\
\hline Raps & & \\
\hline Obst & & \\
\hline Gemüse & & \\
\hline Futterpflanzen & & \\
\hline Sonstige: & & \\
\hline- & & \\
\hline- & & \\
\hline
\end{tabular}

\begin{tabular}{|l|l|l|}
\hline 8. Tierproduktion zum Zeitpunkt der Befragung & freie Stellenkapazitäten \\
\hline Rinder & Anzahl & \\
\hline Milchkühe & & \\
\hline Mastrinder & & \\
\hline Kälber & & \\
\hline Färsen & & \\
\hline Schweine & & \\
\hline Zuchtsauen & & \\
\hline Mastschweine & & \\
\hline Ferkel & & \\
\hline Geflügel & & \\
\hline Schafe & & \\
\hline Pferde & & \\
\hline
\end{tabular}


III. Mechanisierung

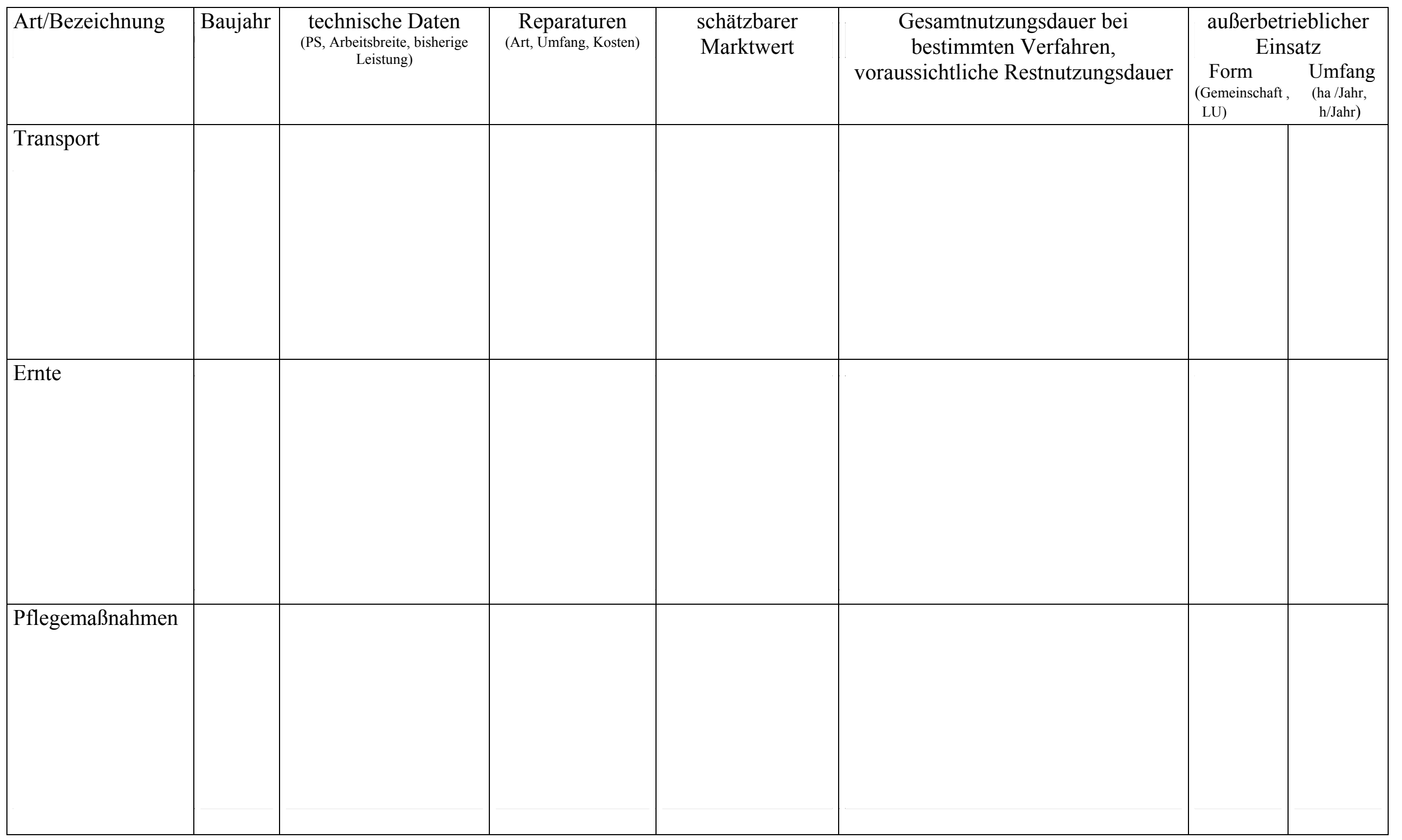




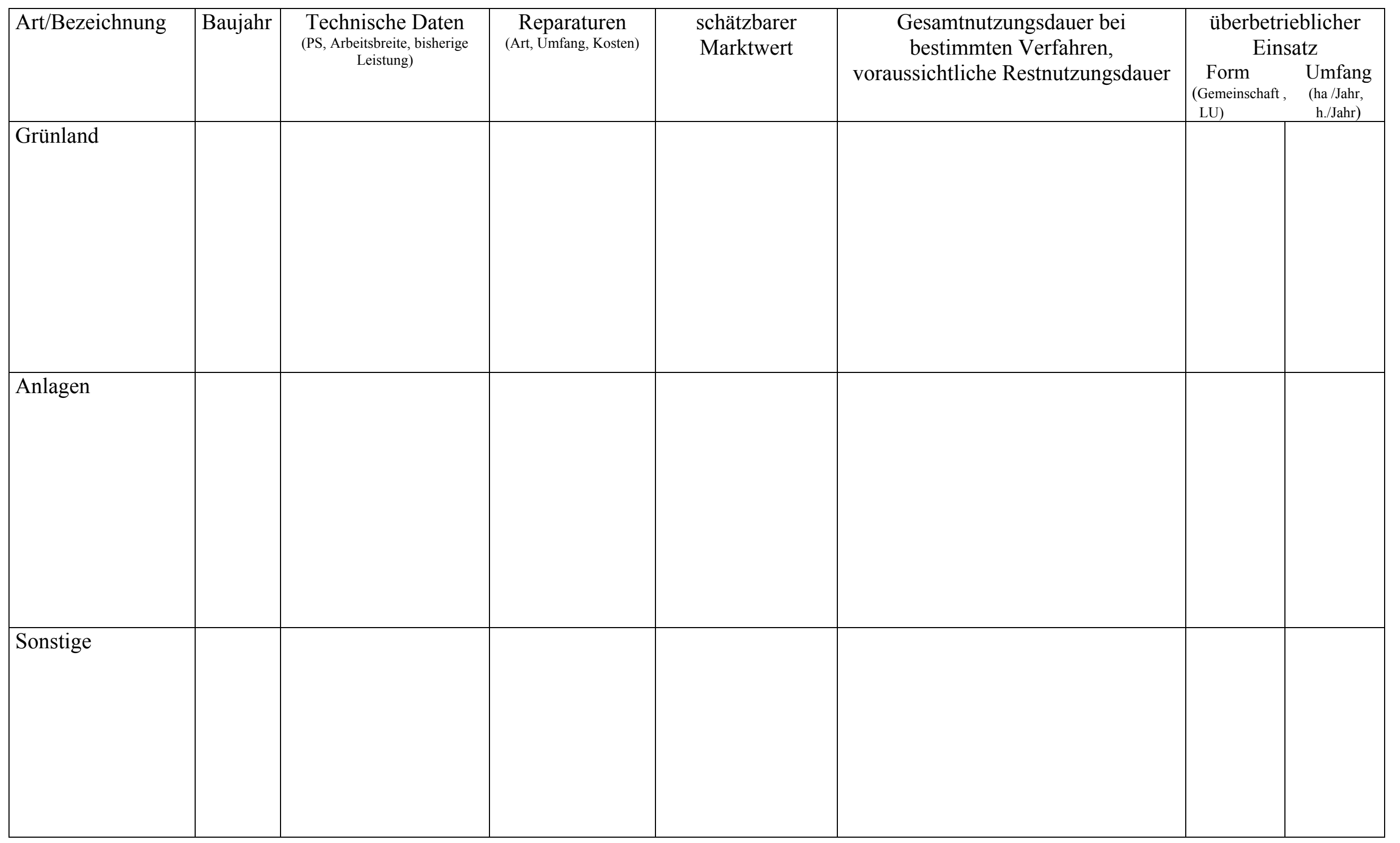


IV. Arbeitsverhältnisse

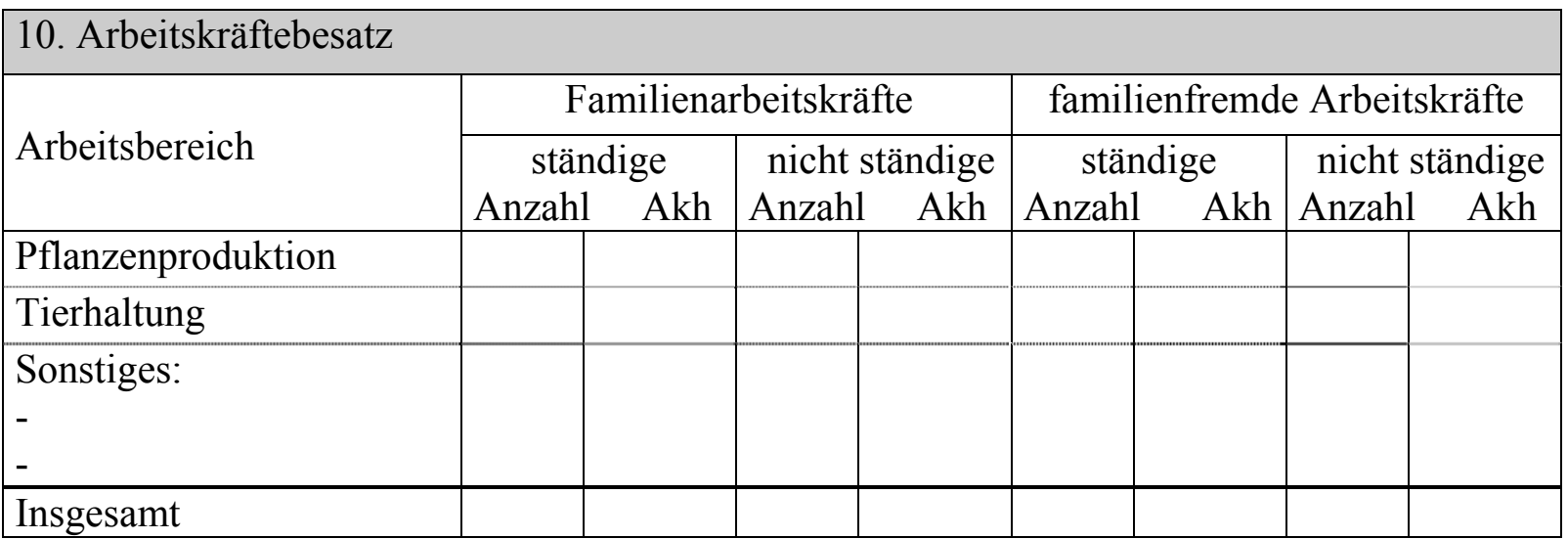

11. Bildet die landwirtschaftliche Tätigkeit die einzige Erwerbstätigkeit?

$\square$ ja $\square$ nein

Falls nein:

a. welche zusätzliche Erwerbstätigkeit wird ausgeübt

b. welches Verhältnis ergibt sich aus den unterschiedlichen Erwerbstätigkeiten

\section{Zur Kooperation}

12. Arbeiten sie mit anderen Betrieben zusammen?
$\square$ ja
$\square$ nein

Falls ja:

welche Bereiche erfasst die Zusammenarbeit und wie hat sie sich entwickelt

Falls nein:

woran liegt das:

$\square$ Sie ziehen einzelbetriebliche Lösungen vor

$\square$ Sie sehen kein Kooperationspotenzial in Ihrem Betrieb

$\square$ die Zusammenarbeit erscheint Ihnen zu riskant

$\square$ es gibt in der Nähe keine entsprechenden Partner 
13. In welchem Maße wird in Ihrer Region die überbetriebliche Zusammenarbeit praktiziert?

14. Wie beurteilen Sie die Chancen der Entwicklung der verschiedenen Formen der Zusammenarbeit in Ihrem Betrieb bzw. Ihrer Region?

- Nachbarschaftshilfe

- Maschinengemeinschaft

- Maschinenring

- Lohnunternehmen

15. Wo liegen aus Ihrer Sicht besondere Hemmnisse in der Entwicklung der Formen der überbetrieblichen Maschinennutzung in Ihrer Region?

16. Wie schätzen Sie die Rolle des Staates, der Beratungsstellen und anderer betroffenen Institutionen in der Förderung der Modernisierung der Landwirtschaft ein?

17. Wie könnte Ihrer Meinung nach die Förderung erfolgen? 


\section{Betriebsentwicklung}

18. Wurden in den letzten 10 Jahren wesentliche Betriebsinvestitionen vorgenommen?
$\square$ ja
$\square$ nein

Falls ja:

in welchem Bereich und in welchem Umfang

19. Wird die Ausdehnung der Produktionsfaktoren vorgesehen?

Falls nein:
$\square \mathrm{ja}$
$\square$ nein

gehen sie bitte zur Frage 21 über.

Falls ja:

welche Bedeutung können in diesem Vorgang folgende Faktoren haben.

sehr hoch hoch gering keine

Boden:

- Kauf

- Pacht

- Kooperation

$\begin{array}{llll}\square & \square & \square & \square \\ \square & \square & \square & \square \\ \square & \square & \square & \square \\ \square & \square & \square & \square\end{array}$

sehr hoch hoch gering keine

Gebäude:

- Errichtung/Kauf

- Pacht

- Kooperation

\begin{tabular}{cccc}
$\square$ & $\square$ & $\square$ & $\square$ \\
$\square$ & $\square$ & $\square$ & $\square$ \\
$\square$ & $\square$ & $\square$ & $\square$ \\
$\square$ & $\square$ & $\square$ & $\square$ \\
\hline sehr hoch & hoch & gering & keine \\
\hline
\end{tabular}

Maschinen:

- Kauf

- Leasing

- Kooperation

$\square$

$\square$

$\square$

$\square$

$\begin{array}{lll}\square & \square & \square \\ \square & \square & \square \\ \square & \square & \square \\ \square & \square & \square\end{array}$

20. Falls es vorgesehen wird, die Erweiterung der Produktionsfaktoren durch Kauf zu realisieren, wie werden die Investitionen finanziert? 
21. Auf welche Aspekte wird in der Fortführung des Betriebes ein besonderer Wert gelegt?

$\square$ Kapitalbeschaffung für weitere Investitionen

$\square$ Mechanisierung

$\square$ Kostensenkung

$\square$ Erschließen außerlandwirtschaftlicher Einkommensquellen $\square$

22. Steht in der nächsten Zeit im Betrieb ein Generationswechsel an?

wie wird er erfolgen

Zur Person des Befragten:

Alter

Ausbildung

Herzlichen Dank für Ihre Unterstützung und gewidmete Zeit! 
A.5 Ergebnisse der empirischen Untersuchung in Polen I Untersuchungsregion Ermland- Masuren

Tabelle 1: Nutzung der LF

\begin{tabular}{|c|c|c|c|c|c|c|c|c|c|c|}
\hline \multirow{2}{*}{$\begin{array}{c}\text { Betriebs- } \\
\mathrm{Nr} .\end{array}$} & \multicolumn{5}{|c|}{ LF in ha } & \multicolumn{5}{|c|}{ Anbauflächen in ha LF } \\
\hline & gesamt & $\mathrm{AL}$ & GL & Sonst. & Pacht & Roggen & Weizen & Triticale & Raps & Mais \\
\hline 1 & 27 & 19 & 8 & 0 & 12,5 & 2 & 6 & 4,5 & 0 & 2 \\
\hline 2 & 27 & 12 & 15 & 0 & 0 & 0 & 1,2 & 5 & 0 & 5 \\
\hline 3 & 29 & 8 & 20 & 1 & 0 & 0 & 1,5 & 6,5 & 0 & 0 \\
\hline 4 & 29 & 8 & 21 & 0 & 6 & 3 & 0 & 1,5 & 0 & 3 \\
\hline 5 & 30 & 30 & 0 & 0 & 0 & 0 & 24 & 0 & 0 & 0 \\
\hline 6 & 38 & 14 & 13 & 10 & 1 & 8 & 0 & 1 & 0 & 4 \\
\hline 7 & 40,5 & 10 & 23 & 0 & 0 & 0 & 0 & 5 & 0 & 0 \\
\hline 8 & 41,5 & 24 & 17,5 & 0 & 14 & 0 & 7,7 & 10 & 6,3 & 0 \\
\hline 9 & 42,5 & 33,5 & 9 & 0 & 6,5 & 0 & 15 & 0 & 8,5 & 4 \\
\hline 10 & 43 & 2,5 & 40,5 & 0 & 12,5 & 0 & 1 & 1,5 & 0 & 0 \\
\hline 11 & 43 & 7 & 36 & 0 & 6,2 & 0 & 0 & 2 & 0 & 5 \\
\hline 12 & 43,5 & 42 & 0 & 0 & 8 & 0 & 22 & 9 & 9,5 & 0 \\
\hline 13 & 50 & 15 & 35 & 0 & 41,5 & 0 & 6 & 5 & 0 & 4 \\
\hline 14 & 52 & 15 & 30 & 0 & 12 & 3 & 0 & 8 & 0 & 5 \\
\hline 15 & 52,6 & 35 & 17 & 0 & 14 & 0 & 34 & 1 & 0 & 0 \\
\hline 16 & 53,6 & 51 & 1 & 0 & 7 & 0 & 27 & 15 & 6 & 0 \\
\hline 17 & 54,8 & 11,5 & 43 & 0 & 21 & 1,2 & 0 & 2,3 & 0 & 8 \\
\hline 18 & 55 & 55 & 0 & 0 & 35 & 0 & 36 & 3 & 16 & 0 \\
\hline 19 & 62 & 62 & 0 & 0 & 26 & 0 & 39 & 3 & 14 & 0 \\
\hline 20 & 68,5 & 65 & 0 & 0 & 28,5 & 0 & 35 & 5,5 & 23 & 0 \\
\hline 21 & 74,6 & 74,6 & 0 & 0 & 26 & 4 & 70 & 0 & 0 & 0 \\
\hline 22 & 69 & 60 & 9 & 0 & 24 & 0 & 40 & 10 & 10 & 0 \\
\hline 23 & 85 & 35 & 50 & 0 & 40 & 0 & 25 & 3,5 & 0 & 6 \\
\hline 24 & 100,6 & 55 & 45,6 & 0 & 30 & 0 & 13 & 28 & 0 & 5 \\
\hline 25 & 106 & 36 & 70 & 0 & 40 & 0 & 15 & 6 & 4 & 3 \\
\hline 26 & 115,5 & 45 & 70 & 0 & 40 & 0 & 0 & 30 & 0 & 15 \\
\hline 27 & 180 & 170 & 10 & 0 & 75 & 5 & 125 & 15 & 20 & 0 \\
\hline 28 & 199 & 178 & 17 & 0 & 150 & 14 & 90 & 25 & 45 & 0 \\
\hline 29 & 335 & 335 & 0 & 0 & 30 & 0 & 240 & 0 & 95 & 0 \\
\hline
\end{tabular}


Tabelle 2: AK- Ausstattung, innere Verkehrslage

\begin{tabular}{|c|c|c|c|c|c|}
\hline $\begin{array}{l}\text { Betriebs- } \\
\text { Nr. }\end{array}$ & AK Gesamt & Fremde AK & $\begin{array}{c}\text { AK pro } 100 \\
\text { ha LF }\end{array}$ & $\begin{array}{c}\text { mittlere Schlaggröße } \\
\text { in ha }\end{array}$ & $\begin{array}{c}\text { mittlere Entfernung } \\
\text { vom Hof in km }\end{array}$ \\
\hline 1 & 2,2 & 0,2 & 8 & 4,6 & 0,5 \\
\hline 2 & 2 & 0 & 7 & 7 & 0,5 \\
\hline 3 & 2,1 & 0,1 & 7 & 29 & 0,3 \\
\hline 4 & 2,1 & 0,1 & 7 & 6,6 & 0,8 \\
\hline 5 & 2,2 & 0,2 & 7 & 8 & 1 \\
\hline 6 & 2,1 & 0 & 6 & 5 & 3,5 \\
\hline 7 & 1,8 & 0 & 4 & 5 & 0,5 \\
\hline 8 & 2,1 & 0,1 & 5 & 6 & 0,5 \\
\hline 9 & 1,4 & 0 & 3 & 4 & 1,5 \\
\hline 10 & 2,1 & 0,1 & 5 & 8,5 & 2 \\
\hline 11 & 2,1 & 0 & 5 & 12 & 1,5 \\
\hline 12 & 1,5 & 0 & 3 & 12 & 0,8 \\
\hline 13 & 3,1 & 1 & 6 & 8 & 0,7 \\
\hline 14 & 2,5 & 0,5 & 5 & 10 & 1,5 \\
\hline 15 & 2,4 & 0 & 5 & 6 & 1,8 \\
\hline 16 & 3 & 0 & 6 & 7 & 0,5 \\
\hline 17 & 2,7 & 0,3 & 5 & 6 & 1,3 \\
\hline 18 & 2 & 0 & 4 & 17 & 0,5 \\
\hline 19 & 2,1 & 0,1 & 3 & 12 & 1 \\
\hline 20 & 2,1 & 0,1 & 3 & 10 & 2,2 \\
\hline 21 & 2,6 & 0,1 & 3 & 8 & 0,7 \\
\hline 22 & 1,8 & 0 & 3 & 5 & 0,8 \\
\hline 23 & 2,3 & 0,3 & 3 & 14 & 0,4 \\
\hline 24 & 3 & 0 & 3 & 20 & 1 \\
\hline 25 & 2,4 & 0,5 & 2 & 18 & 1 \\
\hline 26 & 3,4 & 0,4 & 3 & 14 & 2,8 \\
\hline 27 & 2,3 & 0,3 & 1 & 7 & 1 \\
\hline 28 & 2,5 & 0,5 & 1 & 35 & 6 \\
\hline 29 & 2,3 & 1,3 & 1 & 100 & 2 \\
\hline
\end{tabular}

Quelle: eigene Erhebung 
Tabelle 3: Tierbestände

\begin{tabular}{|c|c|c|c|c|c|c|c|c|c|c|}
\hline \multirow{2}{*}{$\begin{array}{c}\text { Betriebs- } \\
\mathrm{Nr} .\end{array}$} & \multicolumn{3}{|c|}{ Rinder } & \multicolumn{3}{|c|}{ Schweine } & \multicolumn{2}{|c|}{ Pferde } & \multirow[b]{2}{*}{$\begin{array}{l}\text { VE pro } \\
\text { Betrieb }\end{array}$} & \multirow[b]{2}{*}{$\begin{array}{l}\text { VE pro } 100 \\
\text { ha LF }\end{array}$} \\
\hline & Milchkühe & Mastrinder & junges Vieh & Sauen & $\begin{array}{l}\text { Mastschweine } \\
\text { über } 50 \mathrm{~kg}\end{array}$ & $\begin{array}{c}\text { Jungschweine } \\
\text { unter } 50 \mathrm{~kg}\end{array}$ & $\begin{array}{c}\text { über } \\
3 \text { Jahre }\end{array}$ & $\begin{array}{c}\text { unter } \\
3 \text { Jahre }\end{array}$ & & \\
\hline 1 & 10 & 2 & 9 & 0 & 11 & 0 & 0 & 0 & 16 & 60 \\
\hline 2 & 24 & 0 & 25 & 0 & 0 & 0 & 0 & 0 & 15 & 56 \\
\hline 3 & 15 & 7 & 21 & 0 & 1 & 0 & 6 & 5 & 43 & 148 \\
\hline 4 & 16 & 4 & 10 & 0 & 2 & 0 & 0 & 0 & 25 & 86 \\
\hline 5 & 0 & 0 & 0 & 0 & 0 & 0 & 0 & 0 & 0 & 0 \\
\hline 6 & 19 & 1 & 13 & 0 & 0 & 0 & 0 & 0 & 26 & 68 \\
\hline 7 & 0 & 0 & 0 & 0 & 0 & 0 & 7 & 4 & 10 & 25 \\
\hline 8 & 14 & 3 & 20 & 0 & 0 & 0 & 0 & 0 & 28 & 67 \\
\hline 9 & 12 & 4 & 22 & 3 & 6 & 0 & 0 & 0 & 27 & 64 \\
\hline 10 & 17 & 4 & 7 & 0 & 0 & 0 & 0 & 0 & 24 & 56 \\
\hline 11 & 23 & 2 & 19 & 0 & 0 & 0 & 0 & 0 & 34 & 80 \\
\hline 12 & 0 & 0 & 0 & 7 & 1 & 90 & 0 & 0 & 6 & 14 \\
\hline 13 & 26 & 0 & 26 & 0 & 0 & 0 & 0 & 0 & 37 & 74 \\
\hline 14 & 28 & 1 & 30 & 0 & 0 & 5 & 0 & 0 & 45 & 86 \\
\hline 15 & 12 & 2 & 16 & 0 & 0 & 0 & 0 & 0 & 19 & 36 \\
\hline 16 & 0 & 0 & 0 & 12 & 130 & 17 & 0 & 0 & 20 & 37 \\
\hline 17 & 40 & 0 & 14 & 0 & 0 & 0 & 0 & 0 & 12 & 22 \\
\hline 18 & 0 & 0 & 0 & 0 & 0 & 0 & 0 & 0 & 0 & 0 \\
\hline 19 & 0 & 0 & 0 & 1 & 4 & 0 & 0 & 0 & 1 & 2 \\
\hline 20 & 0 & 0 & 0 & 9 & 18 & 52 & 0 & 0 & 6 & 9 \\
\hline 21 & 0 & 0 & 0 & 0 & 140 & 0 & 0 & 0 & 18 & 24 \\
\hline 22 & 0 & 0 & 0 & 0 & 0 & 0 & 0 & 0 & 0 & 0 \\
\hline 23 & 30 & 0 & 30 & 0 & 0 & 0 & 0 & 0 & 44 & 52 \\
\hline 24 & 30 & 0 & 36 & 0 & 3 & 3 & 0 & 0 & 48 & 45 \\
\hline 25 & 30 & 2 & 29 & 0 & 0 & 0 & 0 & 0 & 48 & 42 \\
\hline 26 & 64 & 0 & 45 & 0 & 0 & 0 & 0 & 0 & 89 & 50 \\
\hline 27 & 0 & 0 & 0 & 0 & 0 & 0 & 0 & 0 & 0 & 0 \\
\hline 28 & 0 & 0 & 0 & 0 & 0 & 0 & 0 & 0 & 0 & 0 \\
\hline 29 & 0 & 0 & 0 & 0 & 0 & 0 & 0 & 0 & 0 & 0 \\
\hline
\end{tabular}

Quelle: eigene Erhebung 
Tabelle 4: Maschinenbesatz

\begin{tabular}{|c|c|c|c|c|c|c|c|c|c|c|}
\hline \multirow{2}{*}{$\begin{array}{c}\text { Betriebs- } \\
\text { Nr. }\end{array}$} & \multicolumn{5}{|c|}{ Schlepper } & \multicolumn{5}{|c|}{ Mähdrescher } \\
\hline & Gesamtzahl & $\varnothing$ Alter & PS gesamt & $\mathrm{PS} / 100$ ha LF & $\begin{array}{c}\text { Ø jährliche Auslastung } \\
\text { in } \mathrm{h}\end{array}$ & Gesamtzahl & $\varnothing$ Alter & Arbeitsbreite & $\begin{array}{c}\text { Ø jährliche Auslastung } \\
\text { in h }\end{array}$ & $\begin{array}{c}\text { davon } \\
\text { Lohndrusch in } \mathrm{h}\end{array}$ \\
\hline 1 & 2 & 24 & 135 & 500 & 325 & 1 & 32 & 2 & 40 & 15 \\
\hline 2 & 3 & 15 & 163 & 603 & 275 & 1 & 20 & 4,2 & 90 & 50 \\
\hline 3 & 3 & 20 & 157 & 541 & 193 & 1 & 21 & 2,7 & 80 & 50 \\
\hline 4 & 1 & 19 & 84 & 290 & 270 & 0 & 0 & 0 & 0 & 0 \\
\hline 5 & 2 & 23 & 210 & 700 & 400 & 0 & 0 & 0 & 0 & 0 \\
\hline 6 & 2 & 16 & 75 & 197 & 250 & 0 & 0 & 0 & 0 & 0 \\
\hline 7 & 1 & 20 & 30 & 74 & 300 & 0 & 0 & 0 & 0 & 0 \\
\hline 8 & 1 & 21 & 60 & 145 & 250 & 0 & 0 & 0 & 0 & 0 \\
\hline 9 & 2 & 16 & 142 & 334 & 290 & 1 & 17 & 4,2 & 70 & 0 \\
\hline 10 & 2 & 18 & 90 & 210 & 250 & 1 & 27 & 2,2 & 60 & 0 \\
\hline 11 & 2 & 25 & 90 & 210 & 400 & 0 & 0 & 0 & 0 & 0 \\
\hline 12 & 3 & 22 & 180 & 414 & 260 & 0 & 0 & 0 & 0 & 0 \\
\hline 13 & 3 & 16 & 180 & 360 & 450 & 0 & 0 & 0 & 0 & 0 \\
\hline 14 & 2 & 21 & 90 & 173 & 275 & 1 & 24 & 4,2 & 110 & 95 \\
\hline 15 & 1 & 19 & 45 & 85 & 325 & 0 & 0 & 0 & 0 & 0 \\
\hline 16 & 3 & 28 & 218 & 407 & 235 & 1 & 23 & 4,2 & 80 & 0 \\
\hline 17 & 2 & 13 & 94 & 171 & 250 & 1 & 24 & 4,2 & 100 & 30 \\
\hline 18 & 2 & 14 & 230 & 418 & 275 & 1 & 27 & 3 & 70 & 0 \\
\hline 19 & 2 & 14 & 198 & 320 & 315 & 1 & 27 & 4,2 & 70 & 0 \\
\hline 20 & 2 & 21 & 131 & 192 & 315 & 1 & 25 & 4,2 & 90 & 20 \\
\hline 21 & 3 & 23 & 269 & 360 & 350 & 0 & 0 & 0 & 0 & 0 \\
\hline 22 & 1 & 19 & 250 & 362 & 500 & 0 & 0 & 0 & 0 & 0 \\
\hline 23 & 2 & 20 & 110 & 129 & 250 & 0 & 0 & 0 & 0 & 0 \\
\hline 24 & 3 & 12 & 198 & 197 & 316 & 1 & 16 & 4 & 170 & 110 \\
\hline 25 & 4 & 23 & 300 & 283 & 262 & 0 & 0 & 0 & 0 & 0 \\
\hline 26 & 4 & 10 & 279 & 242 & 212 & 1 & 24 & 4,2 & 100 & 60 \\
\hline 27 & 3 & 25 & 230 & 127 & 317 & 1 & 16 & 4,2 & 180 & 10 \\
\hline 28 & 3 & 24 & 255 & 128 & 400 & 1 & 7 & 4,85 & 250 & 100 \\
\hline 29 & 4 & 14 & 602 & 170 & 600 & 3 & 20 & $3 / 4,5 / 4,8$ & jeweils 190 & 0 \\
\hline
\end{tabular}

Quelle: eigene Erhebung 
Tabelle 5: Kosten des ersten Schleppers

\begin{tabular}{|c|c|c|c|c|c|c|c|c|}
\hline $\begin{array}{c}\text { Betriebs- } \\
\text { Nr. }\end{array}$ & Baujahr & $\begin{array}{l}\mathrm{j} \\
\mathrm{h}\end{array}$ & $\begin{array}{c}\text { Marktwert } \\
\text { ZL }\end{array}$ & $\begin{array}{c}\text { Rest-N } \\
\text { Jahr }\end{array}$ & $\begin{array}{c}\text { FK } \\
\text { ZL/Jahr }\end{array}$ & $\begin{array}{c}\mathrm{VK} \\
\mathrm{ZL} / \mathrm{h}\end{array}$ & $\begin{array}{c}\text { davon Rep. } \\
\text { ZL/h }\end{array}$ & $\begin{array}{c}\mathrm{GK} \\
\mathrm{ZL} / \mathrm{h}\end{array}$ \\
\hline 1 & 1980 & 350 & 20.000 & 5 & 5.200 & 26,5 & 6,5 & 41,4 \\
\hline 2 & 1982 & 350 & 26.000 & 7 & 5.094 & 45 & 6 & 59,6 \\
\hline 3 & 1986 & 150 & 14.000 & 10 & 2.420 & 35 & 12,5 & 51,1 \\
\hline 4 & 2004 & 350 & 75.000 & 15 & 7.850 & 24 & 4,5 & 46,4 \\
\hline 5 & 1984 & 400 & 15.000 & 8 & 2.925 & 24 & 4,3 & 31,3 \\
\hline 6 & 1987 & 200 & 12.000 & 9 & 2.293 & 21,3 & 3,3 & 32,8 \\
\hline 7 & 1989 & 350 & 14.500 & 10 & 2.485 & 17,2 & 5,2 & 24,3 \\
\hline 8 & 1976 & 200 & 8.000 & 3 & 3.507 & 16 & 4 & 33,5 \\
\hline 9 & 1984 & 200 & 12.000 & 5 & 3.360 & 21,3 & 3,3 & 38,1 \\
\hline 10 & 1982 & 350 & 16.000 & 6 & 3.747 & 24 & 3 & 34,7 \\
\hline 11 & 1986 & 400 & 15.000 & 5 & 4.050 & 14 & 2 & 24,1 \\
\hline 12 & 2000 & 400 & 65.000 & 10 & 9.050 & 45 & 5 & 67,6 \\
\hline 13 & 1984 & 450 & 8.000 & 5 & 2.440 & 22 & 3,5 & 27,4 \\
\hline 14 & 1988 & 250 & 14.000 & 4 & 4.520 & 24,5 & 12,5 & 42,6 \\
\hline 15 & 2001 & 400 & 58.000 & 13 & 6.802 & 23 & 4,3 & 40,0 \\
\hline 16 & 1978 & 350 & 18.000 & 3 & 7.140 & 54,6 & 6,6 & 75,0 \\
\hline 17 & 1991 & 350 & 42.000 & 8 & 7.110 & 45,6 & 6,6 & 65,9 \\
\hline 18 & 1992 & 300 & 45.000 & 8 & 7.575 & 47 & 4,7 & 72,3 \\
\hline 19 & 1984 & 350 & 22.000 & 6 & 4.927 & 27 & 5,3 & 41,1 \\
\hline 20 & 1990 & 250 & 35.000 & 6 & 7.483 & 27 & 4,8 & 56,9 \\
\hline 21 & 1986 & 350 & 12.000 & 6 & 2.960 & 19 & 3 & 27,5 \\
\hline 22 & 1985 & 500 & 12.000 & 5 & 3.360 & 23 & 4,4 & 29,7 \\
\hline 23 & 1990 & 300 & 21.000 & 6 & 4.730 & 22 & 4 & 37,8 \\
\hline 24 & 1980 & 450 & 23.000 & 5 & 5.890 & 34,5 & 4,5 & 47,6 \\
\hline 25 & 1989 & 200 & 40.000 & 6 & 8.467 & 24 & 3,2 & 66,3 \\
\hline 26 & 1981 & 100 & 12.000 & 5 & 3.360 & 21,3 & 3,3 & 54,9 \\
\hline 27 & 2004 & 400 & 8.500 & 14 & 1.462 & 37 & 6,4 & 40,7 \\
\hline 28 & 1981 & 500 & 45.000 & 9 & 6.950 & 37,2 & 1,2 & 51,1 \\
\hline 29 & 1993 & 600 & 50.000 & 6 & 10.433 & 61 & 7,1 & 78,4 \\
\hline
\end{tabular}

Quelle: eigene Erhebung und Berechnung 
Tabelle 6: Kosten des zweiten Schleppers

\begin{tabular}{|c|c|c|c|c|c|c|c|c|}
\hline $\begin{array}{c}\text { Betriebs- } \\
\text { Nr. }\end{array}$ & Baujahr & $\begin{array}{l}\mathrm{j} \\
\mathrm{h}\end{array}$ & $\begin{array}{c}\text { Marktwert } \\
\text { ZL }\end{array}$ & $\begin{array}{c}\text { Rest- N } \\
\text { Jahr }\end{array}$ & $\begin{array}{c}\text { FK } \\
\text { ZL/Jahr }\end{array}$ & $\begin{array}{c}\mathrm{VK} \\
\mathrm{ZL} / \mathrm{h}\end{array}$ & $\begin{array}{c}\text { davon Rep. } \\
\text { ZL/h }\end{array}$ & $\begin{array}{l}\mathrm{GK} \\
\mathrm{ZL} / \mathrm{h}\end{array}$ \\
\hline 1 & 1982 & 300 & 12.000 & 5 & 3.360 & 26,5 & 8,6 & 12,3 \\
\hline 2 & 1982 & 200 & 12.000 & 7 & 2.674 & 23,5 & 5,5 & 14,5 \\
\hline 3 & 1989 & 150 & 9.000 & 10 & 1.770 & 28 & 10 & 13,0 \\
\hline 4 & 1978 & 200 & 8.000 & 5 & 2.440 & 30,5 & 12,5 & 13,4 \\
\hline 6 & 1990 & 300 & 17.000 & 10 & 2.810 & 21,3 & 4,8 & 10,4 \\
\hline 7 & 1985 & 250 & 11.000 & 5 & 3.130 & 23,5 & 5,5 & 13,6 \\
\hline 8 & 1985 & 300 & 11.000 & 5 & 3.130 & 25 & 5,5 & 11,5 \\
\hline 9 & 1995 & 380 & 27.000 & 10 & 4.110 & 32,5 & 2,5 & 11,9 \\
\hline 10 & 1991 & 200 & 20.000 & 5 & 5.200 & 49,5 & 3,3 & 27,2 \\
\hline 12 & 1989 & 180 & 25.000 & 7 & 4.921 & 27 & 6 & 28,5 \\
\hline 14 & 1980 & 300 & 10.500 & 5 & 3.015 & 15 & 12,5 & 11,1 \\
\hline 15 & 1983 & 250 & 11.500 & 5 & 3.245 & 15 & 3 & 14,0 \\
\hline 16 & 1985 & 250 & 13.000 & 5 & 3.590 & 25,4 & 7,4 & 15,5 \\
\hline 17 & 1985 & 250 & 20.000 & 5 & 5.200 & 28 & 8,3 & 21,9 \\
\hline 18 & 1990 & 250 & 16.000 & 10 & 2.680 & 21 & 6 & 11,8 \\
\hline 19 & 1984 & 280 & 12.000 & 7 & 2.674 & 23 & 4,8 & 10,6 \\
\hline 20 & 1992 & 380 & 55.000 & 8 & 9.125 & 55 & 6,6 & 25,2 \\
\hline 23 & 1982 & 200 & 8.000 & 5 & 2.440 & 23 & 2,8 & 13,3 \\
\hline 24 & 1980 & 250 & 19.000 & 5 & 4.970 & 28 & 3,8 & 21,0 \\
\hline 25 & 1985 & 150 & 12.000 & 5 & 3.360 & 17 & 4,4 & 23,5 \\
\hline 26 & 1986 & 150 & 13.000 & 6 & 3.157 & 23 & 3,3 & 22,2 \\
\hline 27 & 1986 & 250 & 20.000 & 6 & 4.533 & 23 & 3 & 19,2 \\
\hline 28 & 1978 & 200 & 6.500 & 3 & 2.962 & 28 & 10 & 15,9 \\
\hline 29 & 2002 & 700 & 180.000 & 14 & 18.857 & 68 & 19 & 28,0 \\
\hline
\end{tabular}

Quelle: eigene Erhebung und Berechung 


Tabelle 7: Kosten des dritten Schleppers
\begin{tabular}{|c|c|c|c|c|c|c|c|c|}
\hline $\begin{array}{c}\text { Betriebs- } \\
\text { Nr. }\end{array}$ & Baujahr & $\begin{array}{c}\mathrm{j} \\
\mathrm{h}\end{array}$ & $\begin{array}{c}\text { Marktwert } \\
\text { ZL }\end{array}$ & $\begin{array}{c}\text { Rest-N } \\
\text { Jahr }\end{array}$ & $\begin{array}{c}\text { FK } \\
\text { ZL/Jahr }\end{array}$ & $\begin{array}{c}\text { VK } \\
\text { ZL/h }\end{array}$ & $\begin{array}{c}\text { davon Rep. } \\
\text { ZL/h }\end{array}$ & $\begin{array}{c}\text { GK } \\
\text { ZL/h }\end{array}$ \\
\hline 3 & 1981 & 280 & 7.000 & 7 & 1.810 & 15 & 3 & 7,5 \\
\hline 4 & 1988 & 250 & 15.000 & 9 & 2.717 & 27,5 & 12,5 & 12,0 \\
\hline 10 & 1975 & 200 & 4.500 & 4 & 1.860 & 19 & 4 & 10,4 \\
\hline 12 & 1979 & 200 & 9.000 & 5 & 2.670 & 22 & 10 & 14,5 \\
\hline 16 & 1968 & 100 & 6.000 & 2 & 3.780 & 20 & 8 & 39,0 \\
\hline 17 & 1970 & 150 & 8.000 & 4 & 2.840 & 27 & 20,1 \\
\hline 24 & 1979 & 250 & 8.000 & 4 & 2.840 & 23 & 3 & 12,5 \\
\hline 25 & 2000 & 350 & 125.000 & 10 & 16.850 & 43,2 & & 12 \\
\hline 26 & 1988 & 250 & 15.000 & 8 & 2.925 & 20,3 & 2,3 & 12,8 \\
\hline 27 & 1989 & 300 & 17.000 & 8 & 3.235 & 12 & 1,4 & 11,8 \\
\hline 28 & 1986 & 500 & 13.000 & 5 & 3.590 & 19 & & 4 \\
\hline 29 & 1981 & 600 & 20.000 & 4 & 6.200 & 44 & & 3,6 \\
\hline
\end{tabular}

Quelle: eigene Erhebung und Berechnung

Tabelle 8: Kosten des vierten Schleppers

\begin{tabular}{|c|c|c|c|c|c|c|c|c|}
\hline $\begin{array}{c}\text { Betriebs- } \\
\text { Nr. }\end{array}$ & Baujahr & $\begin{array}{c}\mathrm{j} \\
\mathrm{h}\end{array}$ & $\begin{array}{c}\text { Marktwert } \\
\text { ZL }\end{array}$ & $\begin{array}{c}\text { Rest-N } \\
\text { Jahr }\end{array}$ & $\begin{array}{c}\text { FK } \\
\text { ZL/Jahr }\end{array}$ & $\begin{array}{c}\text { VK } \\
\text { ZL/h }\end{array}$ & $\begin{array}{c}\text { davon Rep. } \\
\text { ZL/h }\end{array}$ & $\begin{array}{c}\text { GK } \\
\text { ZL/h }\end{array}$ \\
\hline 25 & 2003 & 350 & 95.000 & 15 & 9.783 & 31,2 & 8,7 & 29,0 \\
\hline 26 & 1975 & 350 & 7.000 & 4 & 2.560 & 32,5 & 2,5 & 8,4 \\
\hline 29 & 1981 & 500 & 13.000 & 5 & 3.590 & 25 & 2 & 8,2 \\
\hline
\end{tabular}

Quelle: eigene Erhebung und Berechnung 
Tabelle 9: Summe der Schlepperkosten in ZL/h

\begin{tabular}{|c|c|c|c|c|c|}
\hline $\begin{array}{c}\text { Betriebs- } \\
\text { Nr. }\end{array}$ & I & II & III & IV & Summe \\
\hline 1 & 41,4 & 12 & 0 & 0 & 53,4 \\
\hline 2 & 59,6 & 14 & 0 & 0 & 73,6 \\
\hline 3 & 51,1 & 13 & 7,5 & 0 & 71,6 \\
\hline 4 & 46,4 & 10 & 12 & 0 & 68,4 \\
\hline 5 & 31,3 & 0 & 0 & 0 & 31,3 \\
\hline 6 & 32,8 & 10 & 0 & 0 & 42,8 \\
\hline 7 & 24,3 & 14 & 0 & 0 & 38,3 \\
\hline 8 & 33,5 & 12 & 0 & 0 & 45,5 \\
\hline 9 & 38,1 & 12 & 0 & 0 & 50,1 \\
\hline 10 & 34,7 & 27 & 10,4 & 0 & 72,1 \\
\hline 11 & 24,1 & 0 & 0 & 0 & 24,1 \\
\hline 12 & 67,6 & 28 & 14,5 & 0 & 110,1 \\
\hline 13 & 27,4 & 0 & 0 & 0 & 27,4 \\
\hline 14 & 42,6 & 11 & 0 & 0 & 53,6 \\
\hline 15 & 40,0 & 14 & 0 & 0 & 54,0 \\
\hline 16 & 75,0 & 15 & 39 & 0 & 129,0 \\
\hline 17 & 65,9 & 22 & 20,1 & 0 & 108,0 \\
\hline 18 & 72,3 & 12 & 0 & 0 & 84,3 \\
\hline 19 & 41,1 & 11 & 0 & 0 & 52,1 \\
\hline 20 & 56,9 & 25 & 0 & 0 & 81,9 \\
\hline 21 & 27,5 & 0 & 0 & 0 & 27,5 \\
\hline 22 & 29,7 & 0 & 0 & 0 & 29,7 \\
\hline 23 & 37,8 & 13 & 0 & 0 & 50,8 \\
\hline 24 & 47,6 & 21 & 12,5 & 0 & 81,1 \\
\hline 25 & 66,3 & 24 & 49,3 & 29 & 168,6 \\
\hline 26 & 54,9 & 22 & 12,8 & 8,4 & 98,1 \\
\hline 27 & 40,7 & 19 & 11,8 & 0 & 71,5 \\
\hline 28 & 51,1 & 16 & 8,2 & 0 & 75,3 \\
\hline 29 & 78,4 & 28 & 11,4 & 8,2 & 126,0 \\
\hline
\end{tabular}

Quelle: eigene Erhebung und Berechnung
Tabelle 10: Kosten der Mähdrescher

\begin{tabular}{|c|c|c|c|c|c|c|c|c|}
\hline $\begin{array}{c}\text { Betriebs- } \\
\text { Nr. }\end{array}$ & Baujahr & $\begin{array}{c}\mathrm{j} \\
\mathrm{h}\end{array}$ & $\begin{array}{c}\text { Marktwert } \\
\text { ZL }\end{array}$ & $\begin{array}{c}\text { Rest-N } \\
\text { Jahre }\end{array}$ & $\begin{array}{c}\text { FK } \\
\text { ZL/Jahr }\end{array}$ & $\begin{array}{c}\text { VK } \\
\text { ZL/h }\end{array}$ & $\begin{array}{c}\text { davon Rep. } \\
\text { ZL/h }\end{array}$ & $\begin{array}{c}\text { GK } \\
\text { ZL/h }\end{array}$ \\
\hline 1 & 1973 & 40 & 6.000 & 4 & 3.030 & 63,5 & 12,5 & 139 \\
\hline 2 & 1985 & 90 & 28.000 & 8 & 5.690 & 74 & 20 & 137 \\
\hline 3 & 1984 & 80 & 15.000 & 6 & 4.300 & 70 & 25 & 124 \\
\hline 9 & 1989 & 70 & 35.000 & 10 & 5.900 & 58,5 & 4,3 & 143 \\
\hline 10 & 1978 & 60 & 4.000 & 4 & 2.470 & 50 & 5 & 91 \\
\hline 14 & 1981 & 110 & 24.000 & 5 & 6.870 & 81 & 27 & 143 \\
\hline 16 & 1983 & 80 & 25.000 & 5 & 7.100 & 62 & 8 & 151 \\
\hline 17 & 1981 & 100 & 29.000 & 6 & 7.053 & 84 & 30 & 155 \\
\hline 18 & 1978 & 70 & 20.000 & 5 & 5.950 & 62,5 & 14,5 & 148 \\
\hline 19 & 1984 & 60 & 32.000 & 9 & 5.866 & 72,5 & 18,4 & 170 \\
\hline 20 & 1980 & 90 & 30.000 & 6 & 7.250 & 82 & 28 & 163 \\
\hline 24 & 1989 & 170 & 47.000 & 10 & 7.460 & 60 & 5,9 & 104 \\
\hline 26 & 1984 & 100 & 30.000 & 8 & 6.000 & 80 & 25 & 140 \\
\hline 27 & 1987 & 180 & 35.000 & 8 & 6.775 & 70 & 16,7 & 108 \\
\hline 28 & 1998 & 250 & 120.000 & 10 & 16.950 & 95 & 40 & 163 \\
\hline 29 I & 1991 & 190 & 38.000 & 6 & 8.823 & 93 & 36 & 139 \\
\hline 29 II & 1985 & 190 & 30.000 & 5 & 8.250 & 80 & 27 & 123 \\
\hline 29 III & 1979 & 190 & 28.000 & 3 & 11.523 & 64 & 10 & 125 \\
\hline
\end{tabular}

Quelle: eigene Erhebung und Berechnung und Berechnung 
II. Untersuchungsregion Lublin

Tabelle 11: Nutzung der LF

\begin{tabular}{|c|c|c|c|c|c|c|c|c|c|c|c|c|}
\hline \multirow{2}{*}{$\begin{array}{c}\text { Betriebs- } \\
\mathrm{Nr} .\end{array}$} & \multicolumn{5}{|c|}{ LF in ha } & \multicolumn{7}{|c|}{ Anbauflächen in ha } \\
\hline & gesamt & $\mathrm{AL}$ & GL & Sonst. & Pacht & Roggen & Weizen & Triticale & Gerste & Raps & Mais & Rüber \\
\hline 1 & 4 & 4 & 0 & 0 & 0 & 0 & 2 & 0 & 2 & 0 & 0 & 0 \\
\hline 2 & 4,5 & 4,5 & 0 & 0 & 0 & 0 & 2 & 0 & 0 & 2,5 & 0 & 0 \\
\hline 3 & 5 & 5 & 0 & 0 & 0 & 0 & 3 & 0 & 2 & 0 & 0 & 0 \\
\hline 4 & 5,5 & 5,5 & 0 & 0 & 0 & 0 & 1,5 & 0 & 2 & 0 & 0 & 2 \\
\hline 5 & 6,5 & 6 & 0 & 0,5 & 0,7 & 0 & 3 & 0 & 2 & 0 & 0 & 1 \\
\hline 6 & 6,5 & 6,5 & 0 & 0 & 0 & 0 & 3 & 0 & 1 & 2,5 & 0 & 0 \\
\hline 7 & 7,5 & 7 & 0 & 0,5 & 1,5 & 0 & 3 & 0 & 2 & 0 & 0 & 2 \\
\hline 8 & 8 & 7 & 0 & 0 & 0 & 0 & 3 & 0 & 2 & 0 & 0 & 1,5 \\
\hline 9 & 9 & 8 & 0 & 1 & 3,6 & 1,5 & 2 & 0 & 2,5 & 0 & 0 & 2 \\
\hline 10 & 9 & 8 & 0 & 1 & 2 & 0 & 3,5 & 0 & 0 & 3 & 0 & 1,5 \\
\hline 11 & 10,5 & 10 & 0 & 0,5 & 4,5 & 0 & 6,5 & 0 & 1 & 0 & 0 & 2,5 \\
\hline 12 & 12 & 11 & 0 & 1 & 0 & 0 & 6 & 0 & 4 & 0 & 0 & 1 \\
\hline 13 & 14 & 12 & 0 & 2 & 2,5 & 0 & 5,5 & 0 & 4,5 & 0 & 0 & 2 \\
\hline 14 & 16 & 15 & 0 & 1 & 2,5 & 0 & 4,5 & 0 & 3,5 & 4 & 0 & 3 \\
\hline 15 & 18 & 15,5 & 2 & 0,5 & 2,5 & 0 & 5 & 0 & 2,5 & 2 & 6 & 0 \\
\hline 16 & 19,5 & 19 & 0 & 0,5 & 0 & 0 & 9 & 0 & 8 & 0 & 0 & 2 \\
\hline 17 & 20 & 18 & 0 & 2 & 0 & 0 & 6 & 0 & 7 & 0 & 0 & 5 \\
\hline 18 & 21 & 20 & 0 & 1 & 3,5 & 0 & 3,5 & 3,5 & 10 & 0 & 0 & 3 \\
\hline 19 & 22 & 21,5 & 0 & 0,5 & 0 & 0 & 3 & 5,5 & 8 & 0 & 2 & 0,5 \\
\hline 20 & 24 & 23 & 0 & 0 & 0 & 0 & 10 & 1 & 5 & 2 & 0 & 5 \\
\hline 21 & 26,5 & 26 & 0 & 0,5 & 18 & 0 & 9 & 2 & 4 & 7 & 0 & 4 \\
\hline 22 & 30 & 29 & 0 & 1 & 4 & 6 & 12 & 2 & 0 & 4 & 3 & 2 \\
\hline 23 & 40 & 38 & 0 & 2 & 3 & 0 & 18 & 2 & 5 & 0 & 6 & 7 \\
\hline 24 & 43 & 42 & 0 & 1 & 13 & 0 & 15 & 0 & 13 & 3 & 2 & 9 \\
\hline 25 & 52 & 51 & 0 & 1 & 20 & 0 & 18 & 15 & 2 & 0 & 7 & 9 \\
\hline 26 & 62 & 61 & 0 & 1 & 12 & 0 & 20 & 3,5 & 8 & 5,5 & 7 & 17 \\
\hline 27 & 75 & 75 & 0 & 0 & 35 & 0 & 25 & 0 & 25 & 20 & 0 & 5 \\
\hline
\end{tabular}

Quelle: eigene Erhebung 
Tabelle 12: AK- Ausstattung, Verkehrslage

\begin{tabular}{|c|c|c|c|c|c|}
\hline $\begin{array}{c}\text { Betriebs- } \\
\mathrm{Nr} .\end{array}$ & AK Gesamt & Fremde AK & $\begin{array}{c}\text { AK pro } 100 \\
\text { ha LF }\end{array}$ & $\begin{array}{c}\text { mittlere Schlaggröße } \\
\text { in ha }\end{array}$ & $\begin{array}{l}\text { mittlere Entfernung vom Hof } \\
\text { in km }\end{array}$ \\
\hline 1 & 1,5 & 0 & 38 & 2 & 0,5 \\
\hline 2 & 1 & 0 & 22 & 0,8 & 1,5 \\
\hline 3 & 1,2 & 0 & 24 & 1,5 & 1 \\
\hline 4 & 0,5 & 0 & 9 & 1,6 & 1,5 \\
\hline 5 & 0,5 & 0 & 8 & 1 & 3,5 \\
\hline 6 & 0,5 & 0 & 8 & 6 & 0,5 \\
\hline 7 & 1,2 & 0 & 16 & 0,5 & 1,5 \\
\hline 8 & 1,2 & 0 & 15 & 1 & 2 \\
\hline 9 & 1,2 & 0 & 13 & 0,5 & 1,8 \\
\hline 10 & 1 & 0 & 11 & 2 & 0,8 \\
\hline 11 & 1,5 & 0 & 14 & 1,3 & 1,2 \\
\hline 12 & 1 & 0 & 8 & 1,5 & 2,2 \\
\hline 13 & 1,5 & 0 & 11 & 0,8 & 1,5 \\
\hline 14 & 2 & 0 & 13 & 5 & 7 \\
\hline 15 & 2,5 & 0 & 14 & 1,8 & 0,5 \\
\hline 16 & 1,2 & 0 & 6 & 2 & 5 \\
\hline 17 & 1,5 & 0 & 8 & 1,5 & 2 \\
\hline 18 & 1,5 & 0 & 7 & 1,5 & 1 \\
\hline 19 & 2,5 & 0 & 11 & 1,6 & 1,5 \\
\hline 20 & 1,2 & 0 & 5 & 6 & 1 \\
\hline 21 & 2 & 0 & 8 & 2,5 & 1,8 \\
\hline 22 & 2 & 0 & 7 & 3,5 & 2 \\
\hline 23 & 1,5 & 0 & 4 & 3,7 & 0,8 \\
\hline 24 & 2 & 0 & 5 & 6 & 2 \\
\hline 25 & 2 & 0 & 4 & 5,5 & 4 \\
\hline 26 & 2 & 0 & 3 & 3,5 & 3 \\
\hline 27 & 2,5 & 0 & 3 & 6 & 4 \\
\hline
\end{tabular}

Quelle: eigene Erhebung 
Tabelle 13:Tierbestände

\begin{tabular}{|c|c|c|c|c|c|c|c|c|c|c|}
\hline \multirow{2}{*}{$\begin{array}{c}\text { Betriebs- } \\
\text { Nr. }\end{array}$} & \multicolumn{3}{|c|}{ Rinder } & \multicolumn{3}{|c|}{ Schweine } & \multicolumn{2}{|c|}{ Pferde } & \multirow[b]{2}{*}{$\begin{array}{l}\text { VE pro } \\
\text { Betrieb }\end{array}$} & \multirow[b]{2}{*}{$\begin{array}{c}\text { VE pro } 100 \\
\text { ha LF }\end{array}$} \\
\hline & Milchkühe & Mastrinder & $\begin{array}{c}\text { junges } \\
\text { Vieh }\end{array}$ & Sauen & $\begin{array}{l}\text { Mastschweine } \\
\text { über } 50 \mathrm{~kg}\end{array}$ & $\begin{array}{c}\text { Jungschweine } \\
\text { unter } 50 \mathrm{~kg}\end{array}$ & $\begin{array}{c}\text { über } \\
\text { 3 Jahre }\end{array}$ & $\begin{array}{c}\text { unter } \\
3 \text { Jahre }\end{array}$ & & \\
\hline 1 & 0 & 0 & 0 & 0 & 0 & 0 & 0 & 0 & 0 & 0 \\
\hline 2 & 0 & 0 & 0 & 0 & 0 & 0 & 0 & 0 & 0 & 0 \\
\hline 3 & 3 & 0 & 0 & 1 & 0 & 8 & 0 & 0 & 4 & 71 \\
\hline 4 & 1 & 0 & 0 & 2 & 4 & 8 & 0 & 0 & 2 & 45 \\
\hline 5 & 2 & 2 & 2 & 0 & 0 & 0 & 0 & 0 & 4 & 63 \\
\hline 6 & 2 & 0 & 0 & 1 & 0 & 11 & 0 & 0 & 4 & 54 \\
\hline 7 & 2 & 0 & 1 & 0 & 0 & 0 & 0 & 0 & 2 & 31 \\
\hline 8 & 3 & 0 & 3 & 1 & 0 & 0 & 0 & 0 & 4 & 53 \\
\hline 9 & 3 & 0 & 1 & 3 & 0 & 5 & 0 & 0 & 4 & 48 \\
\hline 10 & 0 & 0 & 0 & 0 & 0 & 0 & 0 & 0 & 0 & 0 \\
\hline 11 & 0 & 0 & 0 & 0 & 0 & 0 & 0 & 0 & 0 & 0 \\
\hline 12 & 1 & 0 & 0 & 2 & 3 & 19 & 0 & 0 & 6 & 46 \\
\hline 13 & 4 & 0 & 3 & 1 & 0 & 8 & 0 & 0 & 5 & 38 \\
\hline 14 & 3 & 1 & 2 & 4 & 7 & 30 & 0 & 0 & 7 & 42 \\
\hline 15 & 2 & 2 & 3 & 2 & 14 & 8 & 0 & 0 & 7 & 39 \\
\hline 16 & 0 & 0 & 0 & 5 & 40 & 55 & 0 & 0 & 5 & 26 \\
\hline 17 & 5 & 1 & 1 & 2 & 10 & 0 & 0 & 0 & 8 & 39 \\
\hline 18 & 1 & 1 & 1 & 12 & 50 & 70 & 0 & 0 & 11 & 52 \\
\hline 19 & 10 & 0 & 5 & 4 & 20 & 18 & 5 & 5 & 26 & 117 \\
\hline 20 & 0 & 0 & 0 & 5 & 0 & 30 & 0 & 0 & 2 & 8 \\
\hline 21 & 9 & 7 & 5 & 1 & 5 & 0 & 0 & 0 & 19 & 72 \\
\hline 22 & 0 & 0 & 0 & 0 & 0 & 0 & 0 & 0 & 0 & 0 \\
\hline 23 & 7 & 0 & 1 & 0 & 0 & 0 & 0 & 0 & 7 & 17 \\
\hline 24 & 16 & 0 & 11 & 5 & 20 & 0 & 0 & 0 & 25 & 58 \\
\hline 25 & 4 & 4 & 3 & 50 & 290 & 150 & 0 & 0 & 40 & 77 \\
\hline 26 & 27 & 2 & 19 & 0 & 0 & 0 & 0 & 0 & 39 & 63 \\
\hline 27 & 0 & 0 & 0 & 2 & 9 & 12 & 0 & 0 & 2 & 3 \\
\hline
\end{tabular}

Quelle: eigene Erhebung 
Tabelle 14: Maschinenbesatz

\begin{tabular}{|c|c|c|c|c|c|c|c|c|c|c|}
\hline \multirow{2}{*}{$\begin{array}{c}\text { Betriebs- } \\
\text { Nr. }\end{array}$} & \multicolumn{5}{|c|}{ Schlepper } & \multicolumn{5}{|c|}{ Mähdrescher } \\
\hline & \begin{tabular}{|l|} 
Gesamtzahl \\
\end{tabular} & $\varnothing$ Alter & PS gesamt & PS/100 ha LF & $\begin{array}{c}\varnothing \text { jährliche } \\
\text { Auslastung in h }\end{array}$ & Gesamtzahl & $\varnothing$ Alter & Arbeitsbreite & $\begin{array}{c}\varnothing \text { jährliche } \\
\text { Auslastung in } \mathrm{h} \\
\end{array}$ & $\begin{array}{c}\text { davon } \\
\text { Lohndrusch in ha }\end{array}$ \\
\hline 1 & 1 & 23 & 55 & 1375 & 220 & 1 & 20 & 4,2 & 7 & 25 \\
\hline 2 & 1 & 35 & 30 & 667 & 200 & 0 & 0 & 0 & 0 & 0 \\
\hline 3 & 1 & 35 & 30 & 600 & 250 & 0 & 0 & 0 & 0 & 0 \\
\hline 4 & 1 & 34 & 30 & 545 & 260 & 0 & 0 & 0 & 0 & 0 \\
\hline 5 & 1 & 30 & 30 & 462 & 250 & 0 & 0 & 0 & 0 & 0 \\
\hline 6 & 1 & 15 & 55 & 846 & 300 & 1 & 25 & 4,2 & 15 & 0 \\
\hline 7 & 1 & 32 & 30 & 400 & 280 & 0 & 0 & 0 & 0 & 0 \\
\hline 8 & 1 & 15 & 25 & 313 & 280 & 0 & 0 & 0 & 0 & 0 \\
\hline 9 & 1 & 19 & 55 & 611 & 380 & 0 & 0 & 0 & 0 & 0 \\
\hline 10 & 1 & 25 & 25 & 278 & 250 & 0 & 0 & 0 & 0 & 0 \\
\hline 11 & 1 & 45 & 30 & 286 & 300 & 0 & 0 & 0 & 0 & 0 \\
\hline 12 & 2 & 24 & 150 & 1250 & 200 & 0 & 0 & 0 & 0 & 0 \\
\hline 13 & 2 & 14 & 95 & 679 & 200 & 0 & 0 & 0 & 0 & 0 \\
\hline 14 & 2 & 28 & 100 & 625 & 260 & 0 & 0 & 0 & 0 & 0 \\
\hline 15 & 1 & 33 & 55 & 306 & 280 & 0 & 0 & 0 & 0 & 0 \\
\hline 16 & 2 & 22 & 93 & 477 & 280 & 1 & 27 & 3 & 50 & 0 \\
\hline 17 & 2 & 12 & 90 & 450 & 280 & 1 & 11 & 4,2 & 90 & 50 \\
\hline 18 & 2 & 20 & 110 & 524 & 350 & 1 & 25 & 4,2 & 80 & 40 \\
\hline 19 & 3 & 23 & 165 & 750 & 250 & 1 & 10 & 4,2 & 60 & 30 \\
\hline 20 & 3 & 22 & 165 & 688 & 200 & 1 & 15 & 4,2 & 70 & 30 \\
\hline 21 & 2 & 26 & 145 & 547 & 250 & 1 & 25 & 4,2 & 70 & 25 \\
\hline 22 & 2 & 18 & 120 & 400 & 280 & 1 & 17 & 4,2 & 60 & 0 \\
\hline 23 & 2 & 22 & 168 & 420 & 250 & 0 & 0 & 0 & 0 & 0 \\
\hline 24 & 4 & 13 & 255 & 593 & 250 & 1 & 15 & 4,2 & 55 & 0 \\
\hline 25 & 4 & 12 & 305 & 587 & 150 & 1 & 16 & 4,2 & 100 & 30 \\
\hline 26 & 2 & 3 & 205 & 331 & 500 & 0 & 0 & 0 & 0 & 0 \\
\hline 27 & 2 & 20 & 190 & 253 & 250 & 1 & 21 & 4,2 & 130 & 20 \\
\hline
\end{tabular}

Quelle: eigene Erhebung 
Tabelle 15: Kosten des ersten Schleppers

\begin{tabular}{|c|c|c|c|c|c|c|c|c|}
\hline $\begin{array}{c}\text { Betriebs- } \\
\mathrm{Nr} .\end{array}$ & Baujahr & $\begin{array}{l}\mathrm{j} \\
\mathrm{h}\end{array}$ & Marktwert & $\begin{array}{c}\text { Rest-N } \\
\text { Jahr }\end{array}$ & $\begin{array}{c}\text { FK } \\
\text { ZL/Jahr }\end{array}$ & $\begin{array}{c}\mathrm{VK} \\
\mathrm{ZL} / \mathrm{h}\end{array}$ & $\begin{array}{c}\text { davon Rep. } \\
\text { ZL/h }\end{array}$ & $\begin{array}{c}\mathrm{GK} \\
\mathrm{ZL} / \mathrm{h} \\
\end{array}$ \\
\hline 1 & 1892 & 220 & 13.000 & 5 & 3.640 & 24 & 6 & 40,5 \\
\hline 2 & 1970 & 200 & 8.000 & 4 & 2.890 & 19 & 4 & 33,5 \\
\hline 3 & 1970 & 250 & 8.000 & 4 & 2.890 & 18,2 & 3,2 & 29,8 \\
\hline 4 & 1971 & 260 & 8.500 & 4 & 3.030 & 19,6 & 4,6 & 31,3 \\
\hline 5 & 1970 & 250 & 8.000 & 5 & 2.490 & 18,2 & 3,2 & 28,2 \\
\hline 6 & 1990 & 300 & 17.000 & 8 & 3.285 & 21,3 & 3,3 & 32,3 \\
\hline 7 & 1976 & 280 & 10.000 & 5 & 2.950 & 17,8 & 2,8 & 28,3 \\
\hline 8 & 1990 & 280 & 11.000 & 5 & 3.180 & 14 & 2,1 & 25,4 \\
\hline 9 & 1986 & 380 & 8.000 & 5 & 2.490 & 20,6 & 2,6 & 27,2 \\
\hline 10 & 1980 & 250 & 6.000 & 3 & 2.830 & 14,8 & 2,8 & 26,1 \\
\hline 11 & 1960 & 300 & 4.000 & 3 & 2.103 & 15,3 & 3,3 & 22,3 \\
\hline 12 & 1986 & 200 & 12.000 & 4 & 4.010 & 19 & 7 & 39,1 \\
\hline 13 & 1996 & 250 & 28.000 & 10 & 4.290 & 22 & 4 & 39,2 \\
\hline 14 & 1968 & 220 & 7.000 & 3 & 3.193 & 18 & 3 & 32,5 \\
\hline 15 & 1972 & 280 & 5.000 & 4 & 2.050 & 25 & 7 & 32,3 \\
\hline 16 & 1997 & 300 & 40.000 & 15 & 4.517 & 17,3 & 2,3 & 32,4 \\
\hline 17 & 1979 & 300 & 8.000 & 4 & 2.890 & 14,6 & 2,7 & 24,2 \\
\hline 18 & 1990 & 350 & 38.000 & 8 & 6.540 & 29,8 & 7 & 48,5 \\
\hline 19 & 1991 & 200 & 68.000 & 15 & 7.223 & 26 & 5 & 62,1 \\
\hline 20 & 1995 & 200 & 29.000 & 10 & 4.420 & 24 & 3 & 46,1 \\
\hline 21 & 1974 & 250 & 9.000 & 4 & 3.170 & 24 & 6 & 36,7 \\
\hline 22 & 1993 & 300 & 23.000 & 8 & 4.215 & 23 & 2 & 37,1 \\
\hline 23 & 1988 & 300 & 26.000 & 15 & 3.163 & 24,6 & 6,6 & 35,1 \\
\hline 24 & 1989 & 300 & 32.000 & 10 & 4.810 & 23 & 5 & 39,0 \\
\hline 25 & 1995 & 200 & 30.000 & 15 & 3.550 & 27,5 & 5 & 45,3 \\
\hline 26 & 2001 & 500 & 75.000 & 16 & 7.588 & 20,5 & 3,5 & 35,7 \\
\hline 27 & 1978 & 200 & 8.000 & 4 & 2.890 & 23 & 5 & 37,5 \\
\hline
\end{tabular}

Quelle: eigene Erhebung und Berechnung 
Tabelle 16: Kosten des zweiten Schleppers

\begin{tabular}{|c|c|c|c|c|c|c|c|c|}
\hline $\begin{array}{c}\text { Betriebs- } \\
\text { Nr. }\end{array}$ & Baujahr & $\begin{array}{c}\mathrm{j} \\
\mathrm{h}\end{array}$ & $\begin{array}{c}\text { Marktwert } \\
\text { ZL }\end{array}$ & $\begin{array}{c}\text { Rest-N } \\
\text { Jahr }\end{array}$ & $\begin{array}{c}\text { FK } \\
\text { ZL/Jahr }\end{array}$ & $\begin{array}{c}\text { VK } \\
\text { ZL/h }\end{array}$ & $\begin{array}{c}\text { davon } \\
\text { Rep. ZL/h }\end{array}$ & $\begin{array}{c}\text { GK } \\
\text { ZL/h }\end{array}$ \\
\hline 12 & 1977 & 200 & 13.000 & 5 & 3.640 & 37 & 7 & 55,2 \\
\hline 13 & 1986 & 250 & 11.000 & 6 & 2.813 & 16,8 & 4,8 & 28,1 \\
\hline 14 & 1987 & 280 & 27.000 & 5 & 6.860 & 22,3 & 4,3 & 46,8 \\
\hline 16 & 1989 & 300 & 15.000 & 10 & 2.600 & 15,3 & 3,3 & 24,0 \\
\hline 17 & 1987 & 300 & 27.000 & 6 & 5.960 & 17,3 & 2,3 & 37,2 \\
\hline 18 & 1979 & 310 & 27.000 & 8 & 4.835 & 16,8 & 4,8 & 32,4 \\
\hline 19 & 1978 & 250 & 12.000 & 8 & 2.510 & 20 & 8 & 30,0 \\
\hline 20 & 1987 & 250 & 12.000 & 6 & 3.010 & 22 & 4 & 34,0 \\
\hline 21 & 1985 & 250 & 15.000 & 5 & 4.100 & 25 & 2,5 & 41,4 \\
\hline 22 & 1983 & 260 & 11.000 & 6 & 2.813 & 14 & 2 & 24,8 \\
\hline 23 & 1989 & 200 & 48.000 & 15 & 5.290 & 38 & 7,5 & 64,5 \\
\hline 24 & 1978 & 300 & 6.500 & 4 & 2.470 & 24,5 & 6,5 & 32,7 \\
\hline 25 & 1989 & 80 & 14.500 & 10 & 2.535 & 15 & 3 & 46,7 \\
\hline 26 & 2003 & 500 & 52.000 & 18 & 5.099 & 21 & 3 & 31,2 \\
\hline 27 & 1994 & 300 & 50.000 & 15 & 5.483 & 39 & 9 & 57,3 \\
\hline
\end{tabular}

Quelle: eigene Erhebung und Berechnung

Tabelle 17: Kosten des dritten Schleppers

\begin{tabular}{|c|c|c|c|c|c|c|c|c|}
\hline $\begin{array}{c}\text { Betriebs- } \\
\text { Nr. }\end{array}$ & Baujahr & $\begin{array}{c}\mathrm{j} \\
\mathrm{h}\end{array}$ & $\begin{array}{c}\text { Marktwert } \\
\text { ZL }\end{array}$ & $\begin{array}{c}\text { Rest-N } \\
\text { Jahr }\end{array}$ & $\begin{array}{c}\text { FK } \\
\text { ZL/Jahr }\end{array}$ & $\begin{array}{c}\text { VK } \\
\text { ZL/h }\end{array}$ & $\begin{array}{c}\text { davon } \\
\text { Rep. ZL/h }\end{array}$ & $\begin{array}{c}\text { GK } \\
\text { ZL/h }\end{array}$ \\
\hline 19 & 1979 & 300 & 15.000 & 10 & 2.600 & 21,5 & 5 & 30,2 \\
\hline 20 & 1979 & 250 & 12.000 & 5 & 3.410 & 14,7 & 2,7 & 28,3 \\
\hline 24 & 1997 & 200 & 45.000 & 15 & 5.000 & 22 & 4 & 47,0 \\
\hline 25 & 2005 & 200 & 140.000 & 20 & 11.850 & 30,5 & 0,5 & 89,8 \\
\hline
\end{tabular}

Quelle: eigene Erhebung und Berechnung

Tabelle 18: Kosten des vierten Schleppers

\begin{tabular}{|c|c|c|c|c|c|c|c|c|}
\hline $\begin{array}{c}\text { Betriebs- } \\
\text { Nr. }\end{array}$ & Baujahr & $\begin{array}{c}\mathrm{j} \\
\mathrm{h}\end{array}$ & $\begin{array}{c}\text { Marktwert } \\
\text { ZL }\end{array}$ & $\begin{array}{c}\text { Rest-N } \\
\text { Jahr }\end{array}$ & $\begin{array}{c}\text { FK } \\
\text { ZL/Jahr }\end{array}$ & $\begin{array}{c}\text { VK } \\
\text { ZL/h }\end{array}$ & $\begin{array}{c}\text { davon } \\
\text { Rep. ZL/h }\end{array}$ & $\begin{array}{c}\text { GK } \\
\text { ZL/h }\end{array}$ \\
\hline 24 & 2004 & 250 & 82.000 & 20 & 7.210 & 25,8 & 1,3 & 54,6 \\
\hline 25 & 1986 & 120 & 12.000 & 6 & 3.010 & 23,4 & 5,4 & 48,5 \\
\hline
\end{tabular}

Quelle: eigene Erhebung und Berechnung 
Tabelle 19: Summe der Schlepperkosten in ZL/h

\begin{tabular}{|c|c|c|c|c|c|}
\hline $\begin{array}{c}\text { Betriebs- } \\
\text { Nr. }\end{array}$ & I & II & III & IV & Summe \\
\hline 1 & 40,5 & 0,0 & 0,0 & 0,0 & 40,5 \\
\hline 2 & 33,5 & 0,0 & 0,0 & 0,0 & 33,5 \\
\hline 3 & 29,8 & 0,0 & 0,0 & 0,0 & 29,8 \\
\hline 4 & 31,3 & 0,0 & 0,0 & 0,0 & 31,3 \\
\hline 5 & 28,2 & 0,0 & 0,0 & 0,0 & 28,2 \\
\hline 6 & 32,3 & 0,0 & 0,0 & 0,0 & 32,3 \\
\hline 7 & 28,3 & 0,0 & 0,0 & 0,0 & 28,3 \\
\hline 8 & 25,4 & 0,0 & 0,0 & 0,0 & 25,4 \\
\hline 9 & 27,2 & 0,0 & 0,0 & 0,0 & 27,2 \\
\hline 10 & 26,1 & 0,0 & 0,0 & 0,0 & 26,1 \\
\hline 11 & 22,3 & 0,0 & 0,0 & 0,0 & 22,3 \\
\hline 12 & 39,1 & 55,0 & 0,0 & 0,0 & 94,1 \\
\hline 13 & 39,2 & 28,0 & 0,0 & 0,0 & 67,2 \\
\hline 14 & 32,5 & 47,0 & 0,0 & 0,0 & 79,5 \\
\hline 15 & 32,3 & 0,0 & 0,0 & 0,0 & 32,3 \\
\hline 16 & 32,4 & 24,0 & 0,0 & 0,0 & 56,4 \\
\hline 17 & 24,2 & 37,0 & 0,0 & 0,0 & 61,2 \\
\hline 18 & 48,5 & 32,0 & 0,0 & 0,0 & 80,5 \\
\hline 19 & 62,1 & 30,0 & 30,0 & 0,0 & 122,1 \\
\hline 20 & 46,1 & 34,0 & 28,0 & 0,0 & 108,1 \\
\hline 21 & 36,7 & 41,0 & 0,0 & 0,0 & 77,7 \\
\hline 22 & 37,1 & 25,0 & 0,0 & 0,0 & 62,1 \\
\hline 23 & 35,1 & 64,0 & 0,0 & 0,0 & 99,1 \\
\hline 24 & 39,0 & 33,0 & 47,0 & 55,0 & 174,0 \\
\hline 25 & 45,3 & 47,0 & 90,0 & 48,0 & 230,3 \\
\hline 26 & 35,7 & 31,0 & 0,0 & 0,0 & 66,7 \\
\hline 27 & 37,5 & 57,0 & 0,0 & 0,0 & 94,5 \\
\hline
\end{tabular}

Quelle: eigene Erhebung und Berechnung
Tabelle 20: Kosten der Mähdrescher

\begin{tabular}{|c|c|c|c|c|c|c|c|c|}
\hline $\begin{array}{c}\text { Betriebs- } \\
\text { Nr. }\end{array}$ & Baujahr & $\begin{array}{c}\mathrm{j} \\
\mathrm{h}\end{array}$ & $\begin{array}{c}\text { Marktwert } \\
\text { ZL }\end{array}$ & $\begin{array}{c}\text { Rest-N } \\
\text { Jahr }\end{array}$ & $\begin{array}{c}\text { FK } \\
\text { ZL/Jahr }\end{array}$ & $\begin{array}{c}\text { VK } \\
\text { ZL/h }\end{array}$ & $\begin{array}{c}\text { davon } \\
\text { Rep.ZL/h }\end{array}$ & $\begin{array}{c}\text { GK } \\
\text { ZL/h }\end{array}$ \\
\hline 1 & 1985 & 32 & 23.000 & 8 & 5.565 & 76 & 28 & 249,9 \\
\hline 6 & 1980 & 15 & 19.000 & 6 & 5.737 & 79 & 31 & 461,4 \\
\hline 16 & 1978 & 50 & 15.000 & 5 & 5.450 & 65 & 20 & 174,0 \\
\hline 17 & 1994 & 90 & 37.000 & 12 & 6.193 & 66 & 18 & 134,8 \\
\hline 18 & 1980 & 80 & 20.000 & 5 & 6.600 & 67 & 19 & 149,5 \\
\hline 19 & 1995 & 60 & 45.000 & 12 & 7.100 & 65 & 16,6 & 183,3 \\
\hline 20 & 1991 & 70 & 38.000 & 10 & 6.940 & 66 & 18 & 165,1 \\
\hline 21 & 1980 & 70 & 20.000 & 5 & 6.600 & 73 & 25 & 167,3 \\
\hline 22 & 1988 & 60 & 26.000 & 8 & 6.030 & 70,5 & 22,5 & 171,0 \\
\hline 24 & 1990 & 55 & 42.000 & 10 & 7.460 & 78 & 30 & 213,6 \\
\hline 25 & 1990 & 100 & 42.000 & 10 & 7.460 & 65 & 17 & 139,6 \\
\hline 27 & 1984 & 130 & 26.000 & 8 & 6.030 & 62 & 14 & 108,4 \\
\hline
\end{tabular}

Quelle: eigene Erhebung und Berchnung 